•生物编目・2020年新物种专题

\title{
世界及中国菌物新命名发表概况(2020年)
}

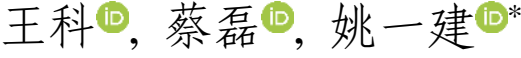

中国科学院微生物研究所菌物标本馆, 北京 100101

摘要：菌物是全球生物多样性最丰富的生物类群之一, 每年有大量新物种被描述和发现, 统计和分析菌物新名称发表数据, 可实时展示菌物分类学的研究热点, 并为学科的未来发展提供参考数据。根据世界菌物名称信息库Fungal Names、Index Fungorum和MycoBank所收录的数据, 2020年全球共发表了4,996个菌物新名称，包括652个新高阶分类单元、2,905 个新种及种 下单元、 1,342 个新组合和 97 个其他名称，是历史上发表菌物新名称数量最多的一年。这些新名称隶属于 12 门 44 纲 173 目 469 科 1,386 属, 盘菌、小型子囊菌、地衣和伞菌是本年度最受关注的类群。来自全球的 1,978 位学者将上述菌物新名称发表在133 个期刊的 885 篇论文和9部专著中，其中669位作者是首次参与新名称发表，是历年来新作者最多的一年。本年度发表的菌物新 物种的模式标本来自世界103个国家和地区，东亚和东南亚是新物种发现的最热点地区，而中国是发现新物种最多的国家， 共发现663种，占全球的 $23 \%$, 是排名第二位的泰国的 2.28 倍。西南地区是我国新物种发现的热点地区，云南、贵州、西藏三 省区本年度发现的新物种数量占全国的 $44.80 \%$ 。除了新物种来源, 中国学者在本年度的菌物分类学研究中取得了令人瞩目的 成果, 333 位中国学者参与发表了 1,271 个菌物新名称, 占全球新名称总数的 $1 / 4$ 。这些菌物新名称隶属于 8 门 24 纲 87 目 209 科 440 属, 发表在 45 个期刊的 258 篇研究论文和 1 部专著中。通过上述研究发现, 全球菌物新物种发现的速度仍在加快, 命名作者和 新名称发表数量在逐步增加, 研究类群规模在逐渐扩大。但菌物物种描述率仍处于较低水平, 持续、高效地发现菌物新物种 将是菌物学者的重点研究方向之一。

关键词：菌物分类学；命名作者；研究类群；新种模式产地；中国贡献

王科, 蔡否, 姚一建 (2021) 世界及中国菌物新名称发表概况(2020年). 生物多样性, 29, 1064-1072. doi: 10.17520/biods.2021202.

Wang K, Cai L, Yao YJ (2021) Annual review on nomenclature novelties of fungi in China and the world (2020). Biodiversity Science, 29, 1064-1072. doi: 10.17520/biods.2021202.

\section{Overview of nomenclature novelties of fungi in the world and China (2020)}

Ke Wang ${ }^{(D)}$, Lei Cai ${ }^{(D)}$, Yijian Yao ${ }^{(\mathbb{D} *}$

Fungarium, Institute of Microbiology, Chinese Academy of Sciences, Beijing 100101

\section{ABSTRACT}

Aims: Fungi have the richest biodiversity on earth, second to insects. Thousands of new fungal species have been discovered and described every year recently. Timely analysis of the published data of new fungal names can show the research hotspots of fungal taxonomy in details and provide reference data for the future development of mycology.

Method: The taxonomic data of new published fungal name were retrieved from the database of Fungal Names, Index Fungorum and MycoBank.

Results: In 2020, at least 4,996 new fungal names including 652 new higher taxa, 2,905 new species and new intraspecific taxa, 1,342 new combinations, and 97 other novelties, were published by 1,978 scholars in 885 articles and 9 monographs all over the world, reaching the historically highest annual record of new fungal names. These new names belong to 12 phyla, 44 classes, 173 orders, 469 families and 1,386 genera, among which ascomycetes had received more attentions. The type specimens of new species were from 103 countries and regions in the world. East and Southeast Asia were the hot spots of new species discovery, while China was the country with the highest number

收稿日期: 2021-05-20; 接受日期: 2021-07-19

基金项目: 中国科学院战略生物资源计划(KFJ-BRP-017-49)

* 通讯作者 Author for correspondence. E-mail: yaoyj@im.ac.cn 
of new species discovered (663 species), accounting for $23 \%$ of the world's total. Besides, China had made remarkable achievements in fungal taxonomy in 2020, with 333 Chinese scholars participating in the publications of 1,271 new fungal names, accounting for about a quarter of the total number in the world.

Conclusion: As shown by the analysis, the discovery of new fungal species is accelerating, with the increased number of new published names and authors. However, the description rate of fungal species is still at a low level, which leaves a long-term and arduous task for mycologists to discover undescribed fungi efficiently.

Key words: fungal taxonomy; author of new fungal name; research group; type locality of new fungal species, China's contribution

近30年来，菌物学家通过多种方法尝试估计全 球菌物的多样性程度。Hawksworth (1991)根据植物 和菌物物种的比率，估算出全球菌物约有 150 万种。 O’Brien等(2005)对环境样本进行了大规模测序分 析, 推测出土壤中菌物物种的数量有约 $350-510$ 万 种。近年, 有研究对植物和菌物的物种比例进行了 修订, 同时考虑了环境中可能的菌物多样性程度, 保守估计出全球的菌物物种数量有 220-380万种 (Hawksworth \& Lücking, 2017)。借助于高通量测序 技术, 研究人员从环境中发现了更多未知的菌物类 群, 进一步提高了菌物多样性的量级。有研究根据 可培养菌物和不可培养菌物的比例, 推测出全球菌 物多样性可能高达 1,170-1,320 万种 (Wu et al, 2019)。而在最新的研究中, 通过对环境样品的菌物 多样性进行分析, 预测出全球菌物物种有 628 万种 (Baldrian et al, 2021)。截至2021年5月, 世界已发现 和描述的菌物仅有 15 万种 (生物物种名录, https://www.catalogueoflife.org/)。根据以上各种方法 的估计数字, 仍有至少 $94 \%$ 的菌物物种未被发现或 描述。本世纪以来, 菌物分类学研究相较于动植物 分类学处于更为活跃的状态, 每年发表新物种的学 者逐渐增多, 新物种描述数量快速增长, 分类学研 究质量也有所提升(Wang et al, 2020)。

中国菌物分类研究始于 20 世纪上半叶, 在经历 了艰难起步和稳步积累阶段后, 目前正处于快速发 展的时期。近 20 年来, 中国菌物分类学对世界的贡 献逐步提升, 模式标本产地来源中国的菌物有 5,458 种, 占全世界同期的 $15.40 \%$ 。近年来, 中国学 者每年发表的新名称数量占到了世界的 $1 / 5$ 左右(王 科等, 2021)。

为了了解世界及中国菌物分类学的研究现状 和热点, 本研究广泛收集世界范围菌物新名称发表 的数据, 对2020年度世界及中国新发表的菌物新名 称进行系统分析和全面梳理, 总结年度菌物新名称
发表的总体情况、命名作者、研究类群、出版物、 模式标本产地、新物种来源基质等信息, 以实时展 示菌物分类学热点, 为学科的未来发展提供参考。

\section{数据来源}

本研究的数据来自于Fungal Names (http://www. fungalinfo.net/)、Index Fungorum (http://www.index fungorum.org/) 和 MycoBank (http://www.mycobank. org/)等 3 个世界菌物名称信息库, 数据获取时间截 止于2021年3月31日。以名称发表年份为条件, 篮选 获得 2020 年发表的菌物名称记录 4,996 条, 每条数 据包含菌物名称、命名作者、分类阶元、发表年份、 发表期刊、模式标本产地、命名法评价等信息。

\section{0年世界荳物新名称发表棉況}

\section{1 总体概述}

2020 年, 全球1,978位学者参与发表了 4,996 个 菌物新名称(附录1), 是历史上发表新名称、新分类 群和新物种数量最多的一年。这些名称包含了 1 个 新纲、1个新亚纲、 21 个新目、 5 个新亚目、 53 个新 科、4个新亚科、300个新属、2个新亚属、96个新 组、 8 个新亚组、 161 个新系、 2,879 个新种、 5 个新 亚种、 11 个新变种和 10 个新变型, 共 3,557 个新分类 单元。此外, 还有 1,342 个新组合、 53 个修订名称、 4 个不合格名称和 40 个不合法名称。 21 世纪以来, 世 界每年发表的菌物新名称数量保持着稳步上升的 趋势, 由2000-2011年均不到3,000个名称, 到2020 年即将超过 5,000 个(图1)。

\section{2 研究类群}

本年度发表的菌物新名称涵盖了真菌 (true fungi)、卵菌(oomycetes)和黏菌(slime mold) 3 个类群, 涉及 $12 门 44$ 纲 173 目 469 科 1,386 属。研究类群覆盖了 世界已知真菌类群 $1 / 10$ 的属和 $2 / 5$ 的科。Beimforde 等(2020)建立了树脂生真菌的新纲 Sareomycetes, 
是本年度所发表的最高等级分类单元。对新分类单 元所属科进行统计发现, 盘菌类(圆盘菌科、盘菌 科)、小型子囊菌(曲霉科、亚隔狍壳科、从赤壳科)、 地衣(黄枝衣科、叶上衣科)和伞菌(丝膜菌科和小脆 柄菇科)是最受研究者关注的类群(表1)。在发表新 分类单元最多的几个类群中, 本年度都有重要文章 或著作发表，对其分类系统进行了全面更新和修 订。Baral等(2020)将其团队30年的研究成果进行总 结, 在世界范围内确定了圆盘菌科有 7 属 470 个物种 (包含 4 个新属和 331 个新种), 使该科的已知物种数 量增加了2.4倍。Houbraken等(2020)全面更新了曲霉 科中曲霉属(Aspergillus)和青霉属(Penicillium) 的分 类系统, 对其属下分类单元进行了重新划分, 建立 了1 个新科、 4 个新属和 141 个属下次级分类单元。

Wächter和Melzer (2020)收集了小脆柄菇科的18,133 条分子序列开展系统发育分析, 并结合形态特征, 提出了该科全新的属级分类体系, 建立了7个新属, 并对该科下 74 个物种做了分类修订。除此之外, 本 年发表的 37 个新属和 198 个新种的科级分类地位未 定, 有待在今后进一步研究。

\section{3 命名作者}

2020 年, 全球有 1,978 位学者参与了世界菌物 新名称的发表, 作者数量仅次于 2016 和2017年, 为 历史第三的年份。其中 669 位作者是首次参与新名 称发表, 是历年来新作者最多的一年。在作者的个 人贡献方面, $44.34 \%$ 的作者 $(877$ 位)仅发表了 1 个新 名称; 而发表 10 个新名称以上的高产作者有 203 位, 占作者总数的约 $1 / 10$ (图2)。

泰国皇太后大学的Kevin Hyde教授是本年度发 表菌物新名称最多的学者, 在58篇文章中参与发表 了 536 个新名称, 占本年度菌物新名称总数的 $10.73 \%$ 。两位来自欧洲的菌物学家Hans-Otto Baral 和Pedro Crous也为本年度的菌物分类学做出了突 出贡献, 分别发表了 449 和 339 个新名称(表2)。

\section{4 名称发表出版物}

2020 年, 来自世界各国的学者将4,996个菌物 新名称发表在 133 个期刊的 885 篇论文和9部专著 中。专著中发表的新名称有 492 个, 仅占新名称总数 的不足 $1 / 10$, 其余 4,504 个名称发表在期刊论文中。 在期刊的选择上, 多数论文发表在菌物学、微生物 学或分类学期刊中, 也有少数名称发表在基因组 学、植物学期刊上。发表文章数最多的是Phytotaxa,

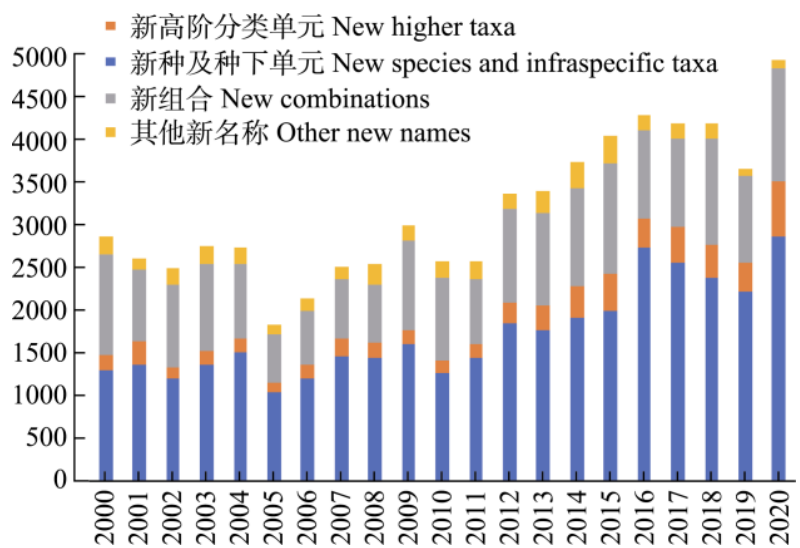

图12000-2020年世界菌物新名称发表数量

Fig. 1 Number of new fungal names published from all over the world between 2000 and 2020

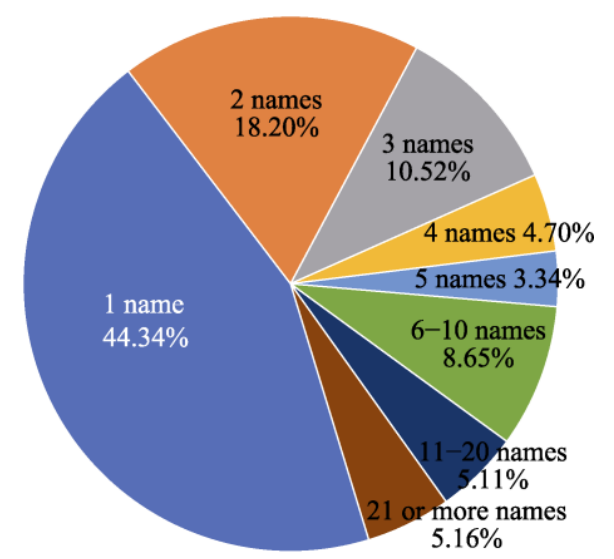

图2 2020年发表不同数量菌物新名称的学者占比

Fig. 2 Magnitude proportion of scholars who published new fungal names in 2020

该期刊立足于高效快速地发表新物种, 所刊载文章 多数描述1-3个新物种, 本年度共发表 97 篇文章 147 个新物种。Fungal Diversity和Studies in Mycology两 种期刊则多刊载针对某个类群系统性研究的文章, 一篇文章往往会发表数十甚至上百个新分类单元 和新名称。Fungal Diversity在本年度刊载了最多的 新名称和新物种, 在发表的 23 篇文章中涉及到 82 个 新高阶分类单元、 405 个新种及种下单元和 226 个新 组合。Studies in Mycology刊载了最多的新高阶分类 单元, 刊载了 12 个新目及亚目、14 个新科和 185 个新 属及属下次级分类单元(图3)。

\section{5 新物种模式产地}

本年度发表的菌物新物种有 2,879 种, 新种的 模式标本来自于世界 7 个大洲的103个国家和地区。 亚欧大陆发现的菌物新物种占全世界的 $60 \%$ 以上, 
表1 2020年发表新分类单元最多的 15 个科

Table 1 Top 15 families in which highest number of new fungal names were published in 2020

\begin{tabular}{lllll}
\hline 科名 & 新属及亚属 & 新种及种下单元 & 新组合 & 总数 \\
Families & New genera and subgenera & New species and infraspecific taxa & New combinations & Total \\
\hline 圆盘菌科 Orbiliaceae & 24 & 327 & 39 & 390 \\
曲霉科 Aspergillaceae & 143 & 47 & 15 & 205 \\
小脆柄菇科 Psathyrellaceae & 34 & 12 & 93 & 139 \\
黄枝衣科 Teloschistaceae & 8 & 48 & 72 & 128 \\
丝膜菌科 Cortinariaceae & 51 & 54 & 5 & 110 \\
亚隔狍壳科 Didymellaceae & 8 & 66 & 21 & 95 \\
从赤壳科 Nectriaceae & 2 & 37 & 47 & 86 \\
叶上衣科 Strigulaceae & 4 & 6 & 72 & 82 \\
盘菌科 Pezizaceae & 10 & 10 & 49 & 69 \\
虫草科 Cordycipitaceae & 4 & 50 & 12 & 66 \\
羽瑚菌科 Pterulaceae & 1 & 0 & 61 & 62 \\
炭角菌科 Xylariaceae & 5 & 22 & 31 & 58 \\
麦角菌科 Clavicipitaceae & 8 & 32 & 14 & 54 \\
茶渍科 Lecanoraceae & 0 & 18 & 33 & 51 \\
\hline
\end{tabular}

表2 2020 年发表 100 个以上菌物新分类单元的学者

Table 2 Scholars who contributed over 100 new fungal names in 2020

\begin{tabular}{|c|c|c|c|c|c|}
\hline $\begin{array}{l}\text { 命名作者 } \\
\text { Author of new names* }\end{array}$ & $\begin{array}{l}\text { 文章数 } \\
\text { Articles }\end{array}$ & $\begin{array}{l}\text { 新高阶分类单元 } \\
\text { New higher taxa }\end{array}$ & $\begin{array}{l}\text { 新种及种下单元 } \\
\text { New species and infraspecific taxa }\end{array}$ & $\begin{array}{l}\text { 新组合 } \\
\text { New combinations }\end{array}$ & $\begin{array}{l}\text { 名称数 } \\
\text { Total new names }\end{array}$ \\
\hline K. D. Hyde & 58 & 72 & 378 & 78 & 536 \\
\hline H. O. Baral & 5 & 26 & 331 & 89 & 449 \\
\hline P. W. Crous & 16 & 62 & 179 & 96 & 339 \\
\hline G. Marson & 2 & 2 & 184 & 4 & 190 \\
\hline J. Houbraken & 10 & 149 & 12 & 24 & 188 \\
\hline J. C. Frisvad & 3 & 146 & 1 & 19 & 168 \\
\hline F. Y. Bai & 1 & 15 & 139 & 9 & 163 \\
\hline Q. M. Wang & 2 & 16 & 130 & 9 & 155 \\
\hline R. Lücking & 14 & 9 & 26 & 115 & 152 \\
\hline S. Y. Kondr. & 6 & 7 & 25 & 104 & 137 \\
\hline L. Cai & 4 & 10 & 89 & 26 & 126 \\
\hline E. Weber & 3 & 18 & 66 & 34 & 118 \\
\hline A. Melzer & 2 & 34 & 2 & 74 & 110 \\
\hline D. Wächt. & 1 & 34 & 0 & 74 & 108 \\
\hline A. H. Li & 2 & 0 & 105 & 1 & 106 \\
\hline
\end{tabular}

*粗体标注为中国作者 $*$ Chinese authors are in bold

而亚洲尤其是东亚和东南亚，是新物种发现的最热 点地区(图4)。地处东亚的中国、日本、韩国一共发 现了 740 个新物种, 占全球同期发表数的 $1 / 4$; 而所 属东南亚的泰国、越南、马来西亚和印度尼西亚等 国，共发现了 324 个新物种，占全球同期的 $11.25 \%$ 。 新物种的来源国家与前几年的趋势类似, 中国
仍是发现新物种最多的国家，本年度共发现663个 菌物新种，占全球新物种总数的近 $1 / 4$ 。此外，本年 度有 6 个国家发现的新种数量超过了 100 个, 分别是 中国、泰国、澳大利亚、美国、巴西和意大利, 6国 一共发现 1,677 个新物种，占全球总数的 $58.25 \%$ (表 3)。这些国家中，中国、澳大利亚、美国和巴西的 


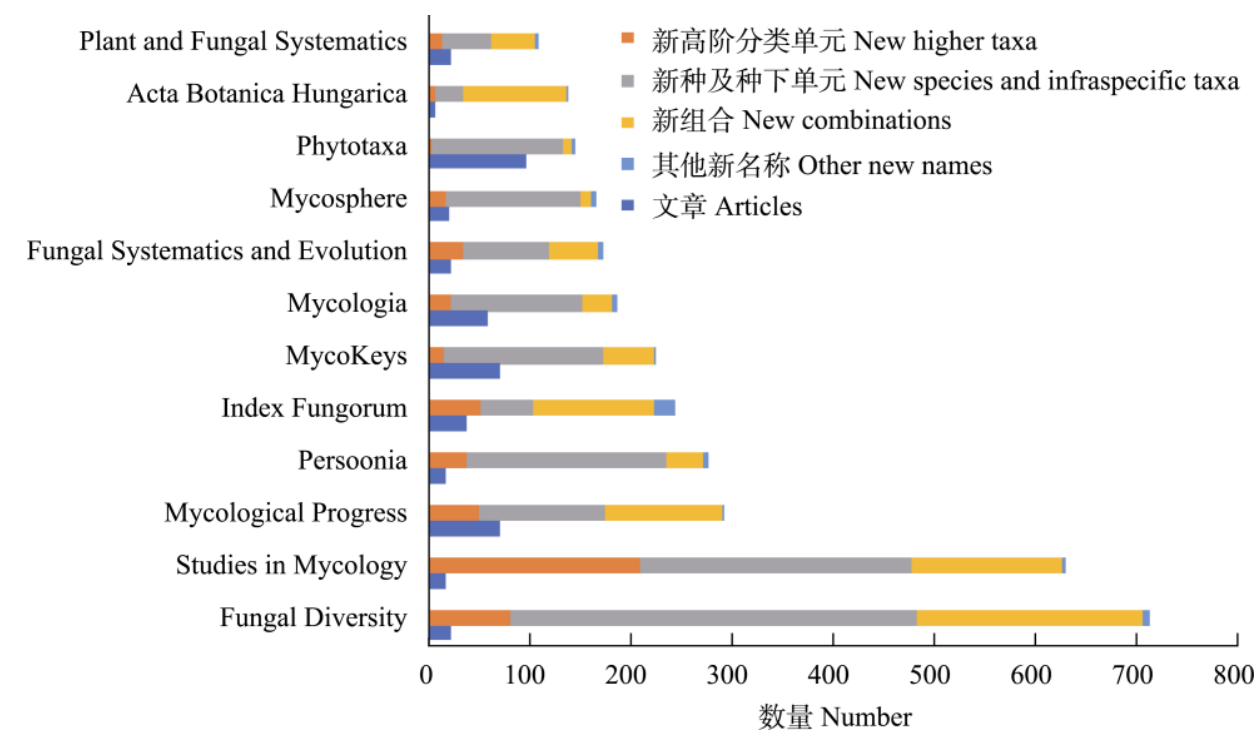

图32020年发表 100 个以上菌物新名称的期刊

Fig. 3 Journals in which scholars have published more than 100 new fungal names in 2020

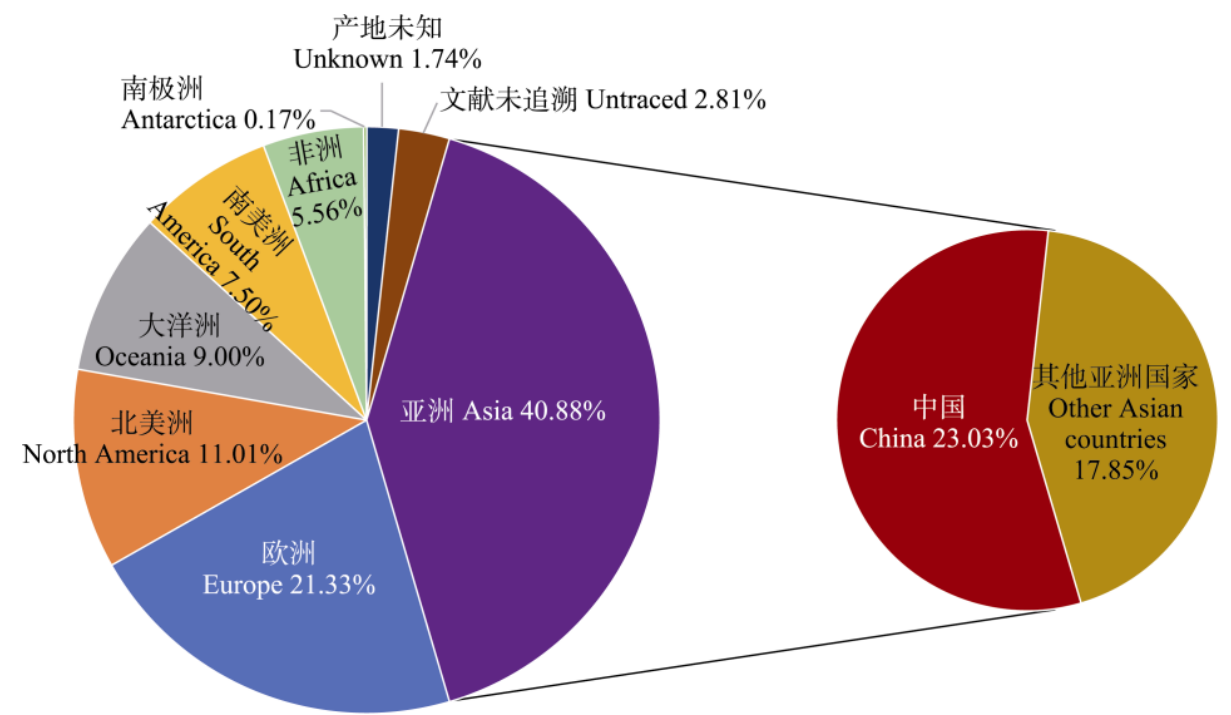

图42020年菌物新物种模式标本产地所属大洲分布

Fig. 4 The number of new fungal species discovered from each continent in 2020

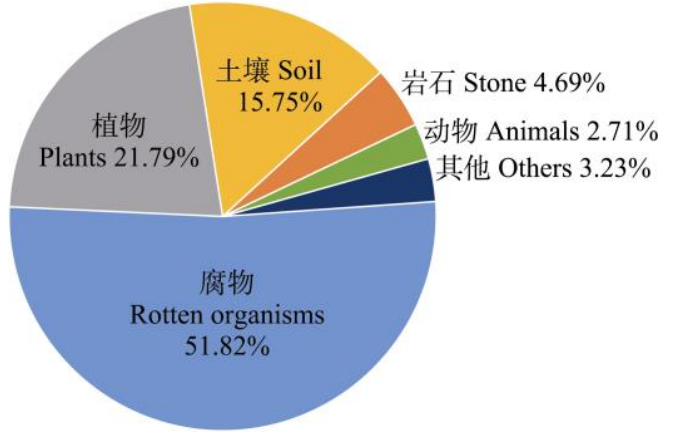

图5 2020年菌物新物种来源基质和/或寄主

Fig. 5 Substrate and/or host origins of new fungal species published in 2020
国土面积辽阔、气候和生态系统丰富多样, 泰国地 处物种多样性热点地区, 而中国、美国和意大利菌 物分类研究基础雄厚。

\section{6 新物种的基质和寄主}

在本年度发表的菌物新物种中, 注明生长基质 或寄主的有959种, 其中生长在腐木、落叶、粪便、 腐殖质等腐物类基质的菌物最多，有497种，占 $51.82 \%$ 。其次, 有 209 种 $(21.79 \%)$ 生长或分离自活体 植物的枝、干、果实等部位; 分离自土壤的菌物新 物种有 151 种 $(15.75 \%)$; 剩余102种寄生在动物身上 
表32020年发表20个以上菌物新物种的国家

Table 3 Countries with more than 20 new fungal species published in 2020

\begin{tabular}{lll}
\hline 国家 & 大洲 & 新物种数 \\
Country & Continent & No. new species \\
\hline 中国 China & 亚洲 Asia & 663 \\
泰国 Thailand & 亚洲 Asia & 291 \\
澳大利亚 Australia & 大洋洲 Oceania & 233 \\
美国 the United States & 北美洲 North America & 221 \\
巴西 Brazil & 南美洲 South America & 147 \\
意大利 Italy & 欧洲 Europe & 122 \\
西班牙 Spain & 欧洲 Europe & 83 \\
南非 South Africa & 非洲 Africa & 62 \\
印度 India & 亚洲 Asia & 55 \\
德国 Germany & 欧洲 Europe & 51 \\
日本 Japan & 亚洲 Asia & 50 \\
俄罗斯 Russia & 欧洲 Europe & 38 \\
加拿大 Canada & 北美洲 North America \\
英国 the United Kingdom & 欧洲 Europe & 34 \\
厄瓜多尔 Ecuador & 南美洲 South America & 31 \\
葡萄牙 Portugal & 欧洲 Europe & 30 \\
荷兰 the Netherlands & 欧洲 Europe & 29 \\
韩国 Republic of Korea & 亚洲 Asia & 27 \\
墨西哥 Mexico & 北美洲 North America & 27 \\
巴基斯坦 Pakistan & 亚洲 Asia & 27 \\
芬兰 Finland & 欧洲 Europe & 23 \\
越南 Vietnam & 亚洲 Asia \\
新西兰 New Zealand & 大洋洲 Oceania & 21 \\
\hline & & 23 \\
\hline
\end{tabular}

或生长于岩石及水体中(图5)。

\section{0 年中国菌物新名称发表概况}

\section{1 总体概述}

中国菌物分类学在 2020 年度延续着近年来高 速发展的趋势。本年度, 有333位中国学者参与发表 了 1,271 个菌物新名称, 是历史上中国学者发表菌 物新名称最多的一年, 分别占本年度世界菌物命名 作者总数的 $16.84 \%$ 和新名称总数的 $25.44 \%$ 。中国学 者所发表的名称有新分类单元 978 个, 包括 3 个新 目、 13 个新科、 83 个新属、 1 个新亚属、 2 个新组、 1 个新亚组、 875 个新种。此外, 中国学者还发表了 276 个新组合和 17 个其他名称。上述名称中, 由中国 学者独立发表的有 627 个 $(49.33 \%)$, 其余 644 个 (50.67\%)由中外学者合作发表, 中外合作发表的比 例较往年有所增加。

\section{2 研究类群}

2020年度, 中国学者所发表的菌物新名称隶属 于 3 界 $8 门 24$ 纲 87 目 209 科 440 属, 科属级别的研究类 群较 2019 年扩大了 $1 / 3$, 也是历史上研究范围最广 泛的一年。本年度中国学者发表的最高等级菌物分 类群是 3 个新目, 包括了 2 个担子菌酵母新目 Rosettozymales和Heitmaniales ( $\mathrm{Li}$ et al, 2020), 以及 1 个水生丝状真菌新目 Neodactylariales (Qiao et al, 2020)。对新分类单元所属的科进行统计，小型子囊 菌(亚隔孢壳科)、地衣(叶上衣科)和木生真菌(革菌 科)是发表新分类单元较多的类群(表4)。中国学者 通过在世界范围内收集材料, 综合形态学和系统发 育分析，对上述类群的分类系统进行了重新修订， 并发现了大量新分类单元。Hou等(2020a)收集了来 自全球 92 个国家、多种生长基质的 1,124 株亚隔狍壳 科菌株, 是迄今为止亚隔狍壳科研究最广泛的取样, 通过多位点系统发育分析和形态比较发现了7 个新 属、40个新种和 21 个新组合。由中外学者合作, 对 叶上衣属(Strigula)地衣的分类系统进行全面梳理, 发现了 1 个新属、3 个新种和近 70 个新组合 (Hongsanan et al, 2020; Jiang et al, 2020)。Yuan等 (2020)通过研究来自中国的标本发现了革菌科小垫 革菌属(Tomentella)新物种 46 个。在发表新名称最多 的 10 个科中，有 6 个科中国学者贡献了超过 $80 \%$ 的 新分类单元，其中革菌科的所有 46 个新分类单元均 为中国学者发表(表4)。

\section{3 命名作者}

在本年度参与新名称发表的中国学者有 333 人, 仅少于 2016 年的 342 人，位居历史第二; 首次参与 新名称发表的有 103 人, 占 $30.93 \%$, 与近年的比例 基本持平。在本年度发表 100 个以上新名称的学者 共有15位，其中4位来自中国(表1)。发表名称最多的 两位学者是从事酵母分类研究的白逢彦研究员和 王启明研究员，分别发表了 163 和 155 个新名称，两 人在本年度合作发表了 2 个新目、3 个新科、 10 个新 属和130多个新种(Li et al, 2020)。此外，子囊菌分类 专家蔡磊研究员本年度参与了4篇分类学文章, 发 表了 1 个新科、 9 个新属、 89 个新种和 26 个新组合 (Hou et al, 2020a,b; Zhao et al, 2020; Zhang et al, 2021)。以上三位学者都是近20年来发表菌物新分类 单元最多的中国学者，持续为中国乃至世界菌物新 物种发现做出了突出的贡献(王科等, 2021)。 
表4 2020年中国学者发表新分类单元最多的10个科

Table 4 Top 10 families in which highest number of new fungal names were published by Chinese scholars in 2020

\begin{tabular}{|c|c|c|c|c|c|}
\hline $\begin{array}{l}\text { 科 } \\
\text { Family }\end{array}$ & $\begin{array}{l}\text { 新属及亚属 New genera } \\
\text { and subgenera }\end{array}$ & $\begin{array}{l}\text { 新种及种下单元 New species } \\
\text { and infraspecific taxa }\end{array}$ & $\begin{array}{l}\text { 新组合 New } \\
\text { combinations }\end{array}$ & $\begin{array}{l}\text { 总数 } \\
\text { Total }\end{array}$ & $\begin{array}{l}\text { 占世界新名称的比例 } \\
\text { Percentage to the world }\end{array}$ \\
\hline 亚隔狍壳科 Didymellaceae & 8 & 52 & 19 & 80 & $84.21 \%$ \\
\hline 叶上衣科 Strigulaceae & 3 & 3 & 70 & 76 & $92.68 \%$ \\
\hline 革菌科 Thelephoraceae & 0 & 46 & 0 & 46 & $100.00 \%$ \\
\hline 虫草科 Cordycipitaceae & 3 & 29 & 10 & 42 & $63.64 \%$ \\
\hline 布勒担菌科 Bulleribasidiaceae & 1 & 31 & 0 & 32 & $91.43 \%$ \\
\hline 新月霉科 Ancylistaceae & 3 & 3 & 20 & 26 & $96.30 \%$ \\
\hline 圆盘菌科 Orbiliaceae & 0 & 15 & 6 & 21 & $5.38 \%$ \\
\hline 间座壳科 Diaporthaceae & 0 & 21 & 0 & 21 & $52.50 \%$ \\
\hline 黑腐皮壳科 Valsaceae & 0 & 20 & 1 & 21 & $84.00 \%$ \\
\hline 刺革菌科 Hymenochaetaceae & 0 & 18 & 2 & 20 & $42.55 \%$ \\
\hline
\end{tabular}

表5 2020年发表自中国各省菌物新物种数量

Table 5 The number of new fungal species published from each Chinese province in 2020

\begin{tabular}{|c|c|c|c|}
\hline $\begin{array}{l}\text { 省 } \\
\text { Province }\end{array}$ & $\begin{array}{l}\text { 新物种数量 } \\
\text { No. new } \\
\text { species }\end{array}$ & $\begin{array}{l}\text { 省 } \\
\text { Province }\end{array}$ & $\begin{array}{l}\text { 新物种数量 } \\
\text { No. of new } \\
\text { species }\end{array}$ \\
\hline 云南 Yunnan & 193 & 河北 Hebei & 6 \\
\hline 贵州 Guizhou & 53 & 湖北 Hubei & 6 \\
\hline 西藏 Xizang & 51 & 青海 Qinghai & 6 \\
\hline 台湾 Taiwan & 49 & 陕西 Shaanxi & 6 \\
\hline 辽宁 Liaoning & 35 & 山西 Shanxi & 6 \\
\hline 海南 Hainan & 34 & 重庆 Chongqing & 5 \\
\hline 广西 Guangxi & 24 & 宁夏 Ningxia & 5 \\
\hline 黑龙江 Heilongjiang & 22 & 浙江 Zhejiang & 5 \\
\hline 吉林 Jilin & 21 & 安徽 Anhui & 4 \\
\hline 广东 Guangdong & 19 & 江苏 Jiangsu & 4 \\
\hline 四川 Sichuan & 16 & 山东 Shandong & 4 \\
\hline 新疆 Xinjiang & 16 & 河南 Henan & 3 \\
\hline 北京 Beijing & 15 & 香港 Hong Kong & 1 \\
\hline 江西 Jiangxi & 11 & 湖南 Hunan & 1 \\
\hline 福建 Fujian & 10 & 天津 Tianjin & 0 \\
\hline 甘肃 Gansu & 9 & 澳门 Macao & 0 \\
\hline $\begin{array}{l}\text { 内蒙古 } \\
\text { Inner Mongolia }\end{array}$ & 9 & 上海 Shanghai & 0 \\
\hline
\end{tabular}

\section{4 名称发表的出版物}

相比全世界的菌物分类学者, 中国学者更倾向 于将新名称发表在期刊论文中。本年 $98.51 \%(1,252$ 个)的名称发表在 45 个期刊的 258 篇研究论文中, 仅 有19个发表在1部专著中(Baral et al, 2020)。在期刊 的选择上, 发表在中文期刊的名称数少于往年, 仅 有 1 个新属、 23 个新种、 4 个新组合和 1 个其他新名
称发表在《菌物学报》《菌物研究》《食用菌学报》 3 个期刊的 16 篇文章中，占期刊发表名称数的 $2.32 \%$ 和文章数的 $6.20 \%$ 。与世界菌物学者的偏好相同，中 国学者在Fungal Diversity和Studies in Mycology两种 期刊发表的新名称最多(分别为 344 和 304 个)，在 Phytotaxa期刊发表的文章数量最多(47篇)。

\section{5 新物种模式产地}

本年度所发表的菌物新物种中, 模式产地来自 中国的有 669 种, 这一数量超过了亚洲其他国家的 总和以及欧洲、美洲、大洋洲等单个大洲所发现新 物种的数量, 占全球的 $23.03 \%$, 是排名第二位泰国 的2.28倍(图4, 表3)。这些模式产自中国的新物种中, $73.54 \%$ (492种)由中国学者发表, 115种由中外学者 合作发表, 剩余62种由外国学者发表。

本年度我国有 31 个省、市、自治区发现了菌物 新物种, 仅有上海、天津、澳门三地没有报道新物 种。西南地区是本年度新物种发现的热点地区, 云 南、贵州、西藏三省区占全国陆地面积的近 $19 \%$, 而 本年度发现的新物种数量占全国的 $44.80 \%$ 。我国东 部沿海和中部地区发现的新物种较少, 各省份数量 多在10个以下。这些地区由于城市化程度较高, 自 然植被较少, 且分类研究的关注度较低, 所以菌物 多样性程度较西南、南方、东北等地较低, 但对这 些地区的菌物多样性调查仍未完成, 需在今后的工 作中进一步关注。

\section{总结与展望}

21 世纪以来, 全球菌物分类研究快速发展, 从 
年均发表不到 3,000 个新名称逐步增长至 4,000 个, 并在 2020 年达到历史最高, 即将迈入年均 5,000 个 的时代。同时，随着新技术的发展和研究的深入， 所估计的全球菌物多样性规模也在逐渐增加。可以 预见, 在未来几十年内, 菌物物种的描述率仍会持 续处于缓慢增长且维持较低的状态。来自全球的菌 物学家需要继续保持并加快菌物新物种描述速度, 逐步提升人类对于菌物多样性的认知。

近年来, 中国无疑是世界菌物分类学的最热点 国家, 发现的新物种数量最多, 中国学者在世界的 贡献已处于领先水平。但相比于欧美国家，我国的 菌物多样性本底尚不清晰, 仍有许多空白区域和薄 弱类群有待探索。作为一个国土辽阔、生态系统多 样的国家, 中国还有大量未知的菌物类群尚未发 现。期待在国内外学者的共同努力下, 能早日将我 国乃至世界的菌物多样性进一步呈现出来。

\section{ORCID}

王科 (iD https://orcid.org/0000-0002-5153-8498

蔡否 (D) https://orcid.org/0000-0003-4084-1202

姚一建 (iD) https://orcid.org/0000-0002-7158-2963

\section{参考文献}

Baldrian P, Větrovský T, Lepinay C, Kohout P (2021) High-throughput sequencing view on the magnitude of global fungal diversity. Fungal Diversity, doi: 10.1007/s13225-021-00472-y.

Baral HO, Weber E, Marson G (2020) Monograph of Orbiliomycetes (Ascomycota) Based on Vital Taxonomy. National Museum of Natural History, Luxembourg.

Beimforde C, Schmidt AR, Rikkinen J, Mitchell JK (2020) Sareomycetes cl. nov.: A new proposal for placement of the resinicolous genus Sarea (Ascomycota, Pezizomycotina). Fungal Systematics and Evolution, 6, 25-37.

Hawksworth DL (1991) The fungal dimension of biodiversity: Magnitude, significance and conservation. Mycological Research, 95, 641-655.

Hawksworth DL, Lücking R (2017) Fungal diversity revisited: 2.2 to 3.8 million species. Microbiology Spectrum, 5, 79-80.

Hongsanan S, Hyde KD, Phookamsak R, Wanasinghe DN, McKenzie EHC, Sarma VV, Lücking R, Boonmee S, Bhat JD, Liu NG, Tennakoon DS, Pem D, Karunarathna A, Jiang SH, Jones GEB, Phillips AJL, Manawasinghe IS, Tibpromma S, Jayasiri SC, Sandamali D, Jayawardena RS, Wijayawardene NN, Ekanayaka AH, Jeewon R, Lu YZ, Phukhamsakda C, Dissanayake AJ, Zeng XY, Luo ZL, Tian Q, Thambugala KM, Dai DQ, Samarakoon MC, Chethana
KWT, Ertz D, Doilom M, Liu JK, Pérez-Ortega S, Suija A, Senwanna C, Wijesinghe SN, Niranjan M, Zhang SN, Ariyawansa HA, Jiang HB, Zhang JF, Norphanphoun C, Silva NI, Thiyagaraja V, Zhang H, Bezerra JDP, Miranda-González R, Aptroot A, Kashiwadani H, Harishchandra D, Sérusiaux E, Abeywickrama PD, Bao DF, Devadatha B, Wu HX, Moon KH, Gueidan C, Schumm F, Bundhun D, Mapook A, Monkai J, Bhunjun CS, Chomnunti P, Suetrong S, Chaiwan N, Dayarathne MC, Yang J, Rathnayaka AR, Xu JC, Zheng JS, Liu G, Feng Y, Xie N (2020) Refined families of Dothideomycetes: Orders and families incertae sedis in Dothideomycetes. Fungal Diversity, 105, 17-318.

Hou LW, Groenewald JZ, Pfenning LH, Yarden O, Crous PW, Cai L (2020a) The Phoma-like dilemma. Studies in Mycology, 96, 309-396.

Hou LW, Hernández-Restrepo M, Groenewald JZ, Cai L, Crous PW (2020b) Citizen science project reveals high diversity in Didymellaceae (Pleosporales, Dothideomycetes). MycoKeys, 65, 49-99.

Houbraken J, Kocsubé S, Visagie CM, Yilmaz N, Wang XC, Meijer M, Kraak B, Hubka V, Bensch K, Samson RA, Frisvad JC (2020) Classification of Aspergillus, Penicillium, Talaromyces and related genera (Eurotiales): An overview of families, genera, subgenera, sections, series and species. Studies in Mycology, 95, 5-169.

Jiang SH, Lücking R, Xavier-Leite AB, Cáceres MES, Aptroot A, Portilla CV, Wei JC (2020) Reallocation of foliicolous species of the genus Strigula into six genera (Lichenized Ascomycota, Dothideomycetes, Strigulaceae). Fungal Diversity, 102, 257-291.

Li AH, Yuan FX, Groenewald M, Bensch K, Yurkov AM, Li K, Han PJ, Guo LD, Aime MC, Sampaio JP, Jindamorakot S, Turchetti B, Inacio J, Fungsin B, Wang QM, Bai FY (2020) Diversity and phylogeny of basidiomycetous yeasts from plant leaves and soil: Proposal of two new orders, three new families, eight new genera and one hundred and seven new species. Studies in Mycology, 96, 17-140.

O'Brien HE, Parrent JL, Jackson JA, Moncalvo JM, Vilgalys R (2005) Fungal community analysis by large-scale sequencing of environmental samples. Applied and Environmental Microbiology, 71, 5544-5550.

Qiao M, Zheng H, Lv R, Yu ZF (2020) Neodactylariales, Neodactylariaceae (Dothideomycetes, Ascomycota): New order and family, with a new species from China. MycoKeys, 73, 69-85.

Wächter D, Melzer A (2020) Proposal for a subdivision of the family Psathyrellaceae based on a taxon-rich phylogenetic analysis with iterative multigene guide tree. Mycological Progress, 19, 1151-1265.

Wang K, Chen SL, Dai YC, Jia ZF, Li TH, Liu TZ, Phurbu D, Mamut R, Sun GY, Bau T, Wei SL, Yang ZL, Yuan HS, Zhang XG, Cai L (2021) Overview of China's nomenclature 
novelties of fungi in the new century (2000-2020). Mycosystema, 40, 1-12. (in Chinese with English abstract) [王科, 陈双林, 戴玉成, 贾泽峰, 李泰辉, 刘铁志, 普布 多吉, 热衣木.马木提, 孙广宇, 图力古尔, 魏生龙, 杨祝 良, 袁海生, 张修国, 蔡磊 (2021) 新世纪中国菌物新名 称发表概况(2000-2020). 菌物学报, 40, 1-12.]

Wang K, Kirk PM, Yao YJ (2020) Development trends in taxonomy, with special reference to fungi. Journal of Systematics and Evolution, 58, 406-412.

Wu B, Hussain M, Zhang WW, Stadler M, Liu XZ, Xiang MC (2019) Current insights into fungal species diversity and perspective on naming the environmental DNA sequences of fungi. Mycology, 10, 127-140.

Yuan HS, Lu X, Dai YC, Hyde KD, Kan YH, Kušan I, He SH, Liu NG, Sarma VV, Zhao CL, Cui BK, Yousaf N, Sun GY, Liu SY, Wu F, Lin CG, Dayarathne MC, Gibertoni TB, Conceição LB, Garibay-Orijel R, Villegas-Ríos M, Salas-Lizana R, Wei TZ, Qiu JZ, Yu ZF, Phookamsak R, Zeng M, Paloi S, Bao DF, Abeywickrama PD, Wei DP, Yang J, Manawasinghe IS, Harishchandra D, Brahmanage RS, Silva NI, Tennakoon DS, Karunarathna A, Gafforov Y, Pem D, Zhang SN, Azevedo Santiago ALCM, Bezerra JDP,
Dima B, Acharya K, Alvarez-Manjarrez J, Bahkali AH, Bhatt VK, Brandrud TE, Bulgakov TS, Camporesi E, Cao T, Chen YX, Chen YY, Devadatha B, Elgorban AM, Fan LF, Du X, Gao L, Gonçalves CM, Gusmão LFP, Huanraluek N, Jadan M, Jayawardena RS, Khalid AN, Langer E, Lima DX, Lima-Júnior NC, Lira CRS, Liu JK, Liu S, Lumyong S, Luo ZL, Matočec N, Niranjan M, Oliveira-Filho JRC, Papp V, Pérez-Pazos E, Phillips AJL, Qiu PL, Ren YH, Ruiz RFC, Semwal KC, Soop K, Souza CAF, Souza-Motta CM, Sun LH, Xie ML, Yao YJ, Zhao Q, Zhou LW (2020) Fungal diversity notes 1277-1386: Taxonomic and phylogenetic contributions to fungal taxa. Fungal Diversity, 104, 1-266.

Zhang ZF, Zhou SY, Eurwilaichitr L, Ingsriswang S, Raza M, Chen Q, Zhao P, Liu F, Cai L (2021) Culturable mycobiota from Karst caves in China II, with descriptions of 33 new species. Fungal Diversity, 106, 29-136.

Zhao P, Qi XH, Crous PW, Duan WJ, Cai L (2020) Gymnosporangium species on Malus: Species delineation, diversity and host alternation. Persoonia, 45, 68-100.

(责任编委: 高程 责任编辑: 时意专)

\section{附录 Supplementary Material}

\section{附录1 世界及中国菌物新名称文献目录(2020)}

Appendix 1 Bibliography of nomenclature novelties of fungi in China and the world (2020)

https://www.biodiversity-science.net/fileup/PDF/2021202-1.pdf 
附录1 Appendix I

\title{
世界及中国菌物新名称文献目录(2020) Bibliography of nomenclature novelties of fungi in China and the world (2020)
}

\author{
王科, 蔡磊, 姚一建 \\ Wang Ke, Cai Lei, Yao Yiian \\ 2021
}




\section{目录}

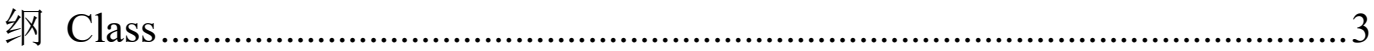

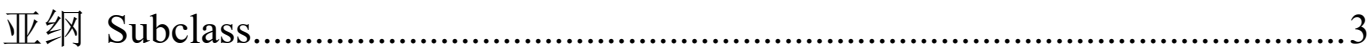

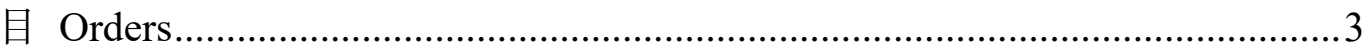

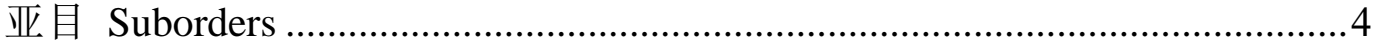

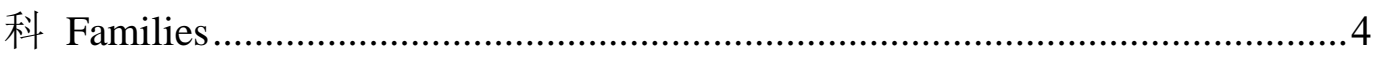

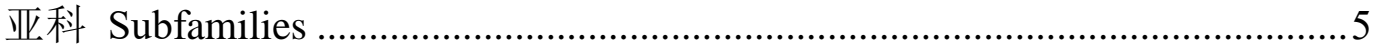

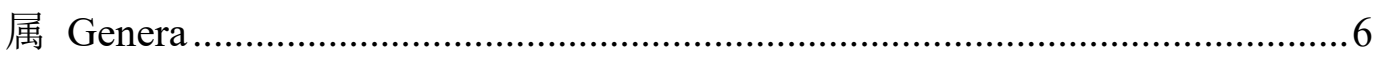



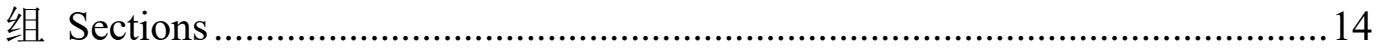

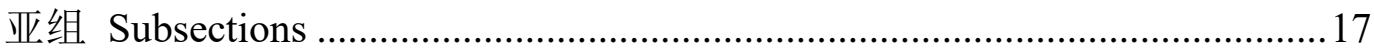

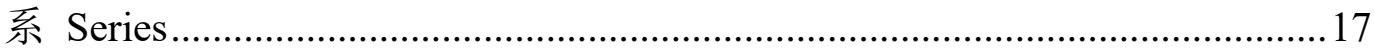

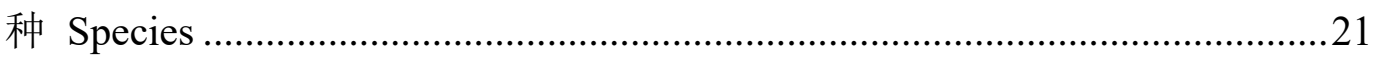

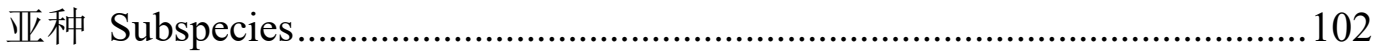

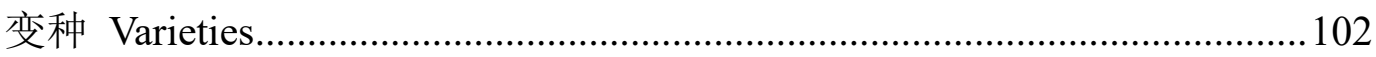

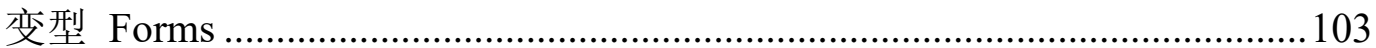

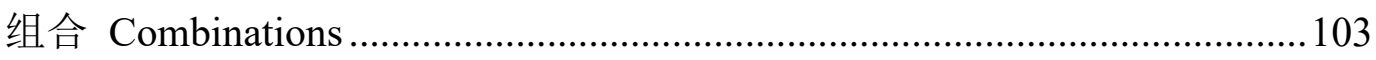

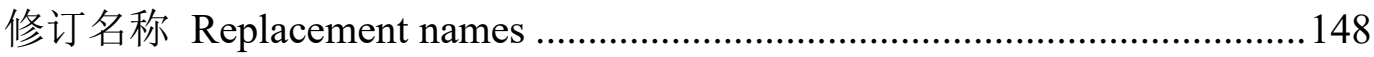



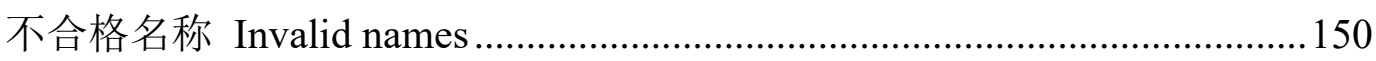




\title{
世界及中国菌物新名称文献目录(2020)
}

\section{Bibliography of nomenclature novelties of fungi in China and the world (2020)}

\author{
同一等级名称按照首字母顺序排列, 标注星号 $\left(^{*}\right)$ 的是中国学者参与发表的 \\ 名称。
}

\section{纲 Class}

Sareomycetes Beimforde, A.R. Schmidt, and Evolution 6: 33 (2020)

Rikkinen \& J.K. Mitch., Fungal Systematics

\section{亚纲 Subclass}

Pisorisporiomycetidae Bundhun, Maharachch. \& K.D. Hyde, Mycosphere 11 (1): 355 (2020)

\section{目 Orders}

Amblyosporida Tokarev \& Issi, Mycosphere 11 (1): 1102 (2020)

Aulographales Crous, Spatafora, Haridas \& I.V. Grig., Stud. Mycol. 96: 151 (2020)

Aureoconidiellales Hern.-Restr. \& Crous, Fungal Systematics and Evolution 6: 2 (2020)

Catabotryales K. D. Hyde, Mycosphere 11 (1): 374 (2020)

Catinellales Ekanayaka, K.D. Hyde \& Ariyawansa, Fungal Diversity 10.1007/s13225-020-00462-6: 50 (2020)

Cintractiellales McTaggart \& R.G. Shivas, Fungal Systematics and Evolution 6: 59 (2020)

Cladosporiales Abdollahz. \& Crous, Stud. Mycol. 95: 390 (2020)

Comminutisporales Abdollahz. \& Crous, Stud. Mycol. 95: 390 (2020)

Coniosporiales Crous, Spatafora, Haridas \& I.V. Grig., Stud. Mycol. 96: 151 (2020)
Eremomycetales Crous, Spatafora, Haridas \& I.V. Grig., Stud. Mycol. 96: 151 (2020)

Eurychasmatales Buaya \& Thines, Philipp. J. Syst. Biol. 14 (1): 8 (2020)

*Heitmaniales Q.M. Wang \& F.Y. Bai, Stud. Mycol. 96: 124 (2020)

Lineolatales Crous, Spatafora, Haridas \& I.V. Grig., Stud. Mycol. 96: 151 (2020)

*Neodactylariales H. Zheng \& Z.F. Yu, MycoKeys 73: 74 (2020)

Neopereziida Tokarev \& Issi, Mycosphere 11 (1): 1102 (2020)

Neophaeothecales Abdollahz. \& Crous, Stud. Mycol. 95: 392 (2020)

Ovavesiculida Tokarev \& Issi, Mycosphere 11 (1): 1103 (2020)

Phaeothecales Abdollahz. \& Crous, Stud. Mycol. 95: 393 (2020)

Racodiales Abdollahz. \& Crous, Stud. Mycol. 95: 393 (2020) 
*Rosettozymales Q.M. Wang \& F.Y. Bai, Stud. Mycol. 96: 117 (2020)

Sareales Beimforde, A.R. Schmidt, Rikkinen
\& J.K. Mitch., Fungal Systematics and Evolution 6: 33 (2020)

\section{亚目 Suborders}

Araucariomycetineae Aime \& McTaggart, Fungal Systematics and Evolution 7: 33 (2020)

Clavariineae Olariaga, Huhtinen, Læssøe, J.H.

Petersen \& K. Hansen, Stud. Mycol. 96: 171 (2020)

Raveneliineae Aime \& McTaggart, Fungal
Systematics and Evolution 7: 35 (2020)

Rogerpetersoniineae Aime \& McTaggart, Fungal Systematics and Evolution 7: 28 (2020)

Skierkineae Aime \& McTaggart, Fungal Systematics and Evolution 7: 31 (2020)

\section{科 Families}

Araucariomycetaceae Aime \& McTaggart, Fungal Systematics and Evolution 7: 33 (2020)

Aureoconidiellaceae Hern.-Restr. \& Crous, Fungal Systematics and Evolution 6: 2 (2020) Callistosporiaceae Vizzini, Consiglio, M. Marchetti \& P. Alvarado, Fungal Diversity 101: 223 (2020)

Catinellaceae Ekanayaka, K.D. Hyde \& Ariyawansa, Fungal Diversity 10.1007/s13225-020-00462-6: 51 (2020)

Clitocybaceae Vizzini, Consiglio \& M. Marchetti, Index Fungorum 462: 1 (2020)

Comminutisporaceae Abdollahz. \& Crous, Stud. Mycol. 95: 391 (2020)

Coniosporiaceae Crous, Spatafora, Haridas \& I.V. Grig., Stud. Mycol. 96: 151 (2020)

Crossopsoraceae Aime \& McTaggart, Fungal Systematics and Evolution 7: 40 (2020)

*Cylindrosympodiaceae Crous, M. Shen \& Y. Zhang ter, Stud. Mycol. 96: 197 (2020)

Dacryonaemataceae J.C. Zamora \& S. Ekman, Persoonia 44: 192 (2020)

Diplogelasinosporaceae Y. Marin \& Stchigel, Microorganisms 8 (9, no. 1430): 10 (2020)

Eriomycetaceae Huanraluek \& Hyde, Fungal Diversity 100: 146 (2020)
*Fasciatisporaceae S.N. Zhang, J.K. Liu \& K.D. Hyde, Fungal Diversity 100: 227 (2020) *Foliocryphiaceae C.M. Tian, N. Jiang \& Crous, Mycologia 112 (2): 281 (2020)

*Gymnosporangiaceae P. Zhao \& L. Cai, Persoonia 45: 79 (2020)

*Heitmaniaceae Q.M. Wang \& F.Y. Bai, Stud. Mycol. 96: 124 (2020)

Induratiaceae Samarak., Thongbai, K.D. Hyde \& M. Stadler, Fungal Diversity 101: 188 (2020)

*Jianyuniaceae Q.M. Wang \& F.Y. Bai, Stud. Mycol. 96: 111 (2020)

Lineolataceae Crous, Spatafora, Haridas \& I.V. Grig., Stud. Mycol. 96: 151 (2020)

Longiostiolaceae Phukhams., Doilom \& K.D. Hyde, Fungal Diversity 102: 43 (2020)

*Mastigosporellaceae C.M. Tian, N. Jiang \& Crous, Mycologia 112 (2): 284 (2020)

Milesinaceae Aime \& McTaggart, Fungal Systematics and Evolution 7: 32 (2020)

Morenoinaceae Hongsanan \& K.D. Hyde, Fungal Diversity 10.1007/s13225-02000462-6: 28 (2020)

Naviculisporaceae Y. Marin \& Stchigel, Microorganisms 8 (9, no. 1430): 12 (2020)

Neoantennariellaceae Abdollahz. \& Crous, 
Stud. Mycol. 95: 404 (2020)

Neobuelliellaceae Hongsanan \& K.D. Hyde, Fungal Diversity 10.1007/s13225-02000462-6: 28 (2020)

*Neodactylariaceae H. Zheng \& Z.F. Yu, MycoKeys 73: 75 (2020)

Neomassarinaceae Mapook \& K.D. Hyde, Fungal Diversity 101: 52 (2020)

Neophaeothecaceae Abdollahz. \& Crous, Stud. Mycol. 95: 392 (2020)

Ochropsoraceae Aime \& McTaggart, Fungal Systematics and Evolution 7: 35 (2020)

Omphalinaceae Vizzini, Consiglio \& M. Marchetti, Index Fungorum 462: 1 (2020)

Patellariopsidaceae Karun., Camporesi \& K.D. Hyde, Frontiers in Microbiology 11 (no. 906): 3 (2020)

Penicillaginaceae Houbraken, Frisvad \& Samson, Stud. Mycol. 95: 9 (2020)

Phyllotopsidaceae Locquin ex Olariaga, Huhtinen, Læssøe, J.H. Petersen \& K. Hansen, Stud. Mycol. 96: 175 (2020)

Protosporangiaceae Leontyev, Stephenson, Schnittler, Shchepin \& Novozhilov, Mycosphere 11 (1): 1103 (2020)

Pseudomassarinaceae Phukhams. \& K.D. Hyde, Fungal Diversity 102: 99 (2020)

*Pygmaeomycetaceae E. Walsh \& N. Zhang, Mycologia

10.1080/00275514.2020.1803649: 8 (2020)

Quaeritorhizaceae Longcore, D.R. Simmons \& T.Y. James, Mycologia 112 (3): 611 (2020)

Radulomycetaceae Leal-Dutra, Dentinger \& G.W. Griff., IMA Fungus 11 (no. 2): 11
(2020)

Readerielliopsidaceae Abdollahz. \& Crous, Stud. Mycol. 95: 407 (2020)

Redonographaceae Lumbsch, Mycosphere 11 (1): 1102 (2020)

Rhizodiscinaceae Crous, Spatafora, Haridas \& I.V. Grig., Stud. Mycol. 96: 151 (2020)

Rogerpetersoniaceae Aime \& McTaggart, Fungal Systematics and Evolution 7: 30 (2020)

*Rosettozymaceae Q.M. Wang \& F.Y. Bai, Stud. Mycol. 96: 117 (2020)

Sarcomyxaceae Olariaga, Huhtinen, Læssøe, J.H. Petersen \& K. Hansen, Stud. Mycol. 96: 177 (2020)

Sareaceae Beimforde, A.R. Schmidt, Rikkinen \& J.K. Mitch., Fungal Systematics and Evolution 6: 25 (2020)

Schizotheciaceae Y. Marin \& Stchigel, Microorganisms 8 (9, no. 1430): 24 (2020)

Skierkaceae Aime \& McTaggart, Fungal Systematics and Evolution 7: 31 (2020)

*Tenuitholiascaceae S.H. Jiang \& J.C. Wei, IMA Fungus 11 (no. 1): 11 (2020)

*Terrestriporiaceae Y.C. Dai, B.K. Cui, F. Wu, Y. Yuan \& Jia J. Chen, Mycosphere 11 (1): 2761 (2020)

*Thyrinulaceae X.Y. Zeng, Hongsanan \& K.D. Hyde, Fungal Diversity 10.1007/s13225020-00462-6: 268 (2020)

Tranzscheliaceae Aime \& McTaggart, Fungal Systematics and Evolution 7: 35 (2020)

Tricladiaceae P.R. Johnst. \& Baschien, Fungal Systematics and Evolution 6: 234 (2020)

\section{亚科 Subfamilies}

Caloplacoideae Arup, Søchting \& Frödén, Plant and Fungal Systematics 65 (2): 528 (2020)

Rubikioideae Lücking, M.Cáceres \& Aptroot, Bryologist 123 (2): 151 (2020)

Teloschistoideae Arup, Søchting \& Frödén,
Plant and Fungal Systematics 65 (2): 552 (2020)

Xanthorioideae Arup, Søchting \& Frödén, Plant and Fungal Systematics 65 (2): 563 (2020) 


\section{属 Genera}

Acidotalaromyces Houbraken, Frisvad \& Samson, Stud. Mycol. 95: 10 (2020)

Aestipascuomyces Stabel, R. Hanafy, Schweitzer, Greif, Aliyu, Flad, D. Young, Lebuhn, Elshahed, Ochsenreither \& N.H. Youssef, Microorganisms 8 (11): 13 (2020) Agriosomyces Hanafy, Lanjekar, Dhakephalkar, T.M. Callaghan, Dagar, G.W. Griff., Elshahed \& N.H. Youssef, Mycologia 112 (6): 1230 (2020)

Ahmadea Aman, Khalid \& Moncalvo, Stud. Fung. 5 (1): 454 (2020)

Aklioshbomyces Hanafy, Lanjekar, Dhakephalkar, T.M. Callaghan, Dagar, G.W. Griff., Elshahed \& N.H. Youssef, Mycologia 112 (6): 1231 (2020)

Albocoprinus Voto, Rivista Micologica Romana, Boll. AMER 110 (2): 90 (2020)

Alishanica Karun., C.H. Kuo \& K.D. Hyde, Mycosphere 11 (1): 460 (2020)

Allocanariomyces Mehrabi, Asgari \& Zare, Mycol. Progr. 19 (12): 1417 (2020)

Allodiatrype Konta \& K.D. Hyde, Mycosphere 11 (1): 247 (2020)

Alternosema J.J. Lipa, Y.S. Tokarev \& I.V. Issi, Parasitology Research 119: 921 (2020)

Amaurodermellus Costa-Rezende, DrechslerSantos \& Góes-Neto, Mycol. Progr. 19 (8): 727 (2020)

Amnocutis K.H. Larss., Syn. Fung. 41: 10 (2020)

Amoeboradix Karpov, López-García, Mamkaeva \& Moreira, Index Fungorum 465: $1(2020)$

Amphosoma Baral, Monogr. Orbiliomycetes: 271 (2020)

*Amyloceraceomyces S.H. He, Fungal Diversity 10.1007/s13225-020-00461-7: 118 (2020)

*Anasporidesmiella K. Zhang, R.F. Castañeda,
Heredia \& Jian Ma, Mycotaxon 135 (4): 723 (2020)

Anthosulcatispora Phukhams. \& K.D. Hyde, Fungal Diversity 10.1007/s13225-02000448-4: 117 (2020)

*Anthracina L. Su, W. Sun \& M.C. Xiang, Journal of Fungi 6 (4, no. 187): 12 (2020)

Aphanodesmium Réblová \& Hern.-Restr., Stud. Mycol. 95: 442 (2020)

*Aquatospora W. Dong, H. Zhang \& K.D. Hyde, Fungal Diversity 10.1007/s13225020-00463-5: 59 (2020)

*Aquihelicascus W. Dong, H. Zhang \& Doilom, Fungal Diversity 10.1007/s13225020-00463-5: 143 (2020)

*Aquimassariosphaeria W. Dong \& Doilom, Fungal Diversity 10.1007/s13225-02000463-5: 106 (2020)

Araucariomyces Aime \& McTaggart, Fungal Systematics and Evolution 7: 34 (2020)

Arboricolonus S. Bien \& Damm, MycoKeys 63: 130 (2020)

Archaeosporites C. Walker, C.J. Harper \& M. Krings, Ann. Bot. 126: 921 (2020)

Areotheca Y. Marín \& Stchigel, Microorganisms 8 (9, no. 1430): 13 (2020)

Ascospirella Houbraken, Frisvad \& Samson, Stud. Mycol. 95: 11 (2020)

Atrophysma T. Sprib., Lichenologist 52 (2): 85 (2020)

Aureoconidiella Hern.-Restr. \& Crous, Fungal Systematics and Evolution 6: 2 (2020)

Babosia D.G. Knapp, Zagyva, Trappe \& Kovács, Mycologia 112 (4): 811 (2020)

Baidera Ertz \& Diederich, Plant and Fungal Systematics 65 (1): 22 (2020)

Batnamyces Noumeur, Mycol. Progr. 19 (6): 495 (2020)

*Begerowomyces Q.M. Wang \& F.Y. Bai, Stud. Mycol. 96: 117 (2020) 
Bellamyces Crous, Coppins \& U. Braun, Stud. Mycol. 96: 199 (2020)

Benniella Vandepol \& Bonito, Fungal Diversity 10.1007/s13225-020-00455-5: 15 (2020)

Bergerella Diederich \& Lawrey, Bryologist 123 (2): 159 (2020)

Bhagirathimyces S.M. Singh \& S.K. Singh, Fungal Diversity 100: 93 (2020)

*Boekhoutia Q.M. Wang \& F.Y. Bai, Stud. Mycol. 96: 110 (2020)

Bolbea Buaya \& Thines, Fungal Systematics and Evolution 6: 131 (2020)

Brijax M. Krings \& C.J. Harper, Review of Palaeobotany and Palynology 281 (no. 104287): 4 (2020)

Britzelmayria D. Wächt. \& A. Melzer, Mycol. Progr. 19 (11): 1213 (2020)

Bryoclavula H. Masumoto \& Y. Degawa, Mycol. Progr. 19 (7): 708 (2020)

Bryopistillaria Olariaga, Huhtinen, Læssøe, J.H. Petersen \& K. Hansen, Stud. Mycol. 96: 177 (2020)

Bryorbilia Baral \& E. Rubio, Monogr. Orbiliomycetes: 302 (2020)

Burrowsia Fryday \& I. Medeiros, S. Afr. J. Bot. 132: 473 (2020)

Cambaraspora Bojkoa, Behringer, Molere, Strattond \& Reisingerd, J. Invert. Path. 171 (no. 107345): 8 (2020)

*Camporesiomyces D.P. Wei, Wanas. \& K.D. Hyde, Fungal Diversity 100: 139 (2020)

Candelinella S.Y. Kondr., Acta bot. hung. 62 (3-4): 301 (2020)

Candolleomyces D. Wächt. \& A. Melzer, Mycol. Progr. 19 (11): 1228 (2020)

Canomyces Rahul Sharma \& Shouche, Mycopathologia 185 (1): 76 (2020)

Capellomyces Hanafy, Lanjekar, Dhakephalkar, T.M. Callaghan, Dagar, G.W. Griff., Elshahed \& N.H. Youssef, Mycologia 112 (6): 1231 (2020)

*Capillidium B. Huang \& Y. Nie, MycoKeys
66: 62 (2020)

Carneothele Fryday, T. Sprib. \& M. Svenss., Lichenologist 52 (2): 91 (2020)

*Catenuliconidia N.G. Liu \& K.D. Hyde, Fungal Diversity 10.1007/s13225-02000461-7: 97 (2020)

Chromelosporiopsis Hennebert, Mycotaxon 135 (3): 688 (2020)

Chromolaenicola Mapook \& K.D. Hyde, Fungal Diversity 101: 20 (2020)

Chromolaenomyces Mapook \& K.D. Hyde, Fungal Diversity 101: 96 (2020)

*Cladocillium Chun-Hao Chen \& R. Kirschner, Mycol. Progr. 19 (9): 840 (2020)

*Claviformispora X.L. Xu \& C.L. Yang, MycoKeys 70: 8 (2020)

Commelinaceomyces E. Tanaka, Mycologia 112 (3): 655 (2020)

*Corylicola Wijesinghe, Camporesi, Yong Wang bis \& K.D. Hyde, Biodiversity Data Journal 8 (e55957): 7 (2020)

Cristataspora Robledo \& Costa-Rezende, Mycol. Progr. 19 (8): 733 (2020)

Cryolevonia A. Pontes, Ruethi, B. Frey \& J.P. Samp., Int. J. Syst. Evol. Microbiol. 10.1099/ijsem.0.004040: 3 (2020)

*Cryphognomonia C.M. Tian \& N. Jiang, MycoKeys 69: 79 (2020)

Cyberloma Minter, IMI Descr. Fungi Bact. 225 (no. 2241): 3 (2020)

Diabolocovidia Crous, Persoonia 44: 331 (2020)

*Dimorphoma L.W. Hou, L. Cai \& Crous, Stud. Mycol. 96: 388 (2020)

*Ectodidymella L.W. Hou, L. Cai \& Crous, Stud. Mycol. 96: 340 (2020)

Elaiopezia Van Vooren, Ascomycete.org 12 (4): 185 (2020)

Elongaticollum D.S. Tennakoon, C.H. Kuo \& K.D. Hyde, MycoKeys 70: 70 (2020)

*Elongaticonidia W.J. Li \& Camporesi \& K.D. Hyde, Fungal Diversity 100: 514 (2020)

Emmanuelia Ant. Simon, Lücking \& Goffinet, 
Plant and Fungal Systematics 65 (1): 82 (2020)

Entomortierella Vandepol \& Bonito, Fungal Diversity 10.1007/s13225-020-00455-5: 15 (2020)

Erichansenia S.Y. Kondr., Kärnefelt \& A. Thell, Acta bot. hung. 62 (1-2): 112 (2020)

Eriocamporesia R.H. Perera, Samarak. \& K.D. Hyde, Fungal Diversity 100: 165 (2020)

Eriomyces Huanraluek, Thambugala \& K.D. Hyde, Fungal Diversity 100: 146 (2020)

Evansstolkia Houbraken, Frisvad \& Samson, Stud. Mycol. 95: 13 (2020)

*Fagicola Crous, M. Shen \& Y. Zhang ter, Stud. Mycol. 96: 217 (2020)

Fasciodontia Yurchenko \& Riebesehl, Mycol. Progr. 19 (2): 178 (2020)

*Flagellostrigula Lücking, S.H. Jiang \& Sérus., Fungal Diversity 10.1007/s13225020-00462-6: 131 (2020)

*Flavocillium H. Yu, Y.B. Wang, Y. Wang, Q. Fan \& Zhu L. Yang, Fungal Diversity 103: 19 (2020)

*Fraxinicola Crous, M. Shen \& Y. Zhang ter, Stud. Mycol. 96: 217 (2020)

*Fuscohilum Crous, M. Shen \& Y. Zhang ter, Stud. Mycol. 96: 200 (2020)

*Fusiformiseptata W. Dong, H. Zhang \& K.D. Hyde, Fungal Diversity 10.1007/s13225020-00463-5: 195 (2020)

Fusiformispora Phukhams. \& K.D. Hyde, Fungal Diversity 10.1007/s13225-02000448-4: 8 (2020)

Gamsomyces Hern.-Restr. \& Réblová, Stud. Mycol. 95: 448 (2020)

*Gamszarea Z.F. Zhang \& L. Cai, Fungal Diversity 10.1007/s13225-020-00453-7: 69 (2020)

Ghazallomyces Hanafy, Lanjekar, Dhakephalkar, T.M. Callaghan, Dagar, G.W. Griff., Elshahed \& N.H. Youssef, Mycologia 112 (6): 1232 (2020)

*Glaciozyma Turchetti, Connell, Thomas-Hall
\& Boekhout ex M. Groenew. \& Q.M. Wang, Stud. Mycol. 96: 133 (2020)

Globosporidium Y. Yakovleva, E. Nassonova, N. Lebedeva, O. Lanzoni, G. Petroni, A. Potekhin, E. Sabaneyeva, Parasitology 147 (9): 957-971 (2020)

Gobabebomyces Crous, Persoonia 45: 273 (2020)

Grigorovia Gouliamova \& Dimitrov, Comptes Rendus de l'Académie Bulgare des Sciences 73 (1): 55 (2020)

Gryganskiella Vandepol \& Bonito, Fungal Diversity 10.1007/s13225-020-00455-5: 16 (2020)

Guayaquilia R.F. Castañeda, Magdana, D. Sosa \& Hern.-Restr., Mycotaxon 135 (3): 506 (2020)

Halocryptosphaeria Dayarath., Devadatha, V.V. Sarma \& K.D. Hyde, Mycosphere 11 (1): 136 (2020)

Halocryptovalsa Dayar. \& K.D. Hyde, Cryptog. Mycol. 41 (3): 49 (2020)

Halotestudina Dayarathne \& K.D. Hyde, Mycosphere 11 (1): 69 (2020)

Hansenopezia Matočec, I. Kušan \& Jadan, Fungal Diversity 10.1007/s13225-02000461-7: 64 (2020)

Hantamomyces Crous, Persoonia 45: 297 (2020)

Hausknechtia D. Wächt. \& A. Melzer, Mycol. Progr. 19 (11): 1234 (2020)

Helgardiomyces Crous, Fungal Systematics and Evolution 7: 83 (2020)

Hirticrusta Matozaki, T. Hatt. \& Sotome, Mycoscience 61 (5): 243 (2020)

*Hispidopannaria Elvebakk, S.G. Hong \& C.H. Park, Mycol. Progr. 19 (11): 1356 (2020)

*Hyalinostachys C.G. Lin \& K.D. Hyde, Mycosphere 11 (1): 877 (2020)

Ionopezia Van Vooren, Ascomycete.org 12 (4): 187 (2020)

Italiofungus Crous, Fungal Systematics and 
Evolution 6: 194 (2020)

Jennwenomyces Goh \& C.H. Kuo, Mycol. Progr. 19 (9): 874 (2020)

Joblinomyces Hanafy, Lanjekar, Dhakephalkar, T.M. Callaghan, Dagar, G.W. Griff., Elshahed \& N.H. Youssef, Mycologia 112 (6): 1233 (2020)

Jocatoa R. Miranda, Bryologist 123 (2): 136 (2020)

Juncomyces Crous, Persoonia 44: 333 (2020)

Kaarikia C. Mayers \& T.C. Harr., Mycologia 112 (6): 1126 (2020)

Kalmanago T. Denchev, Denchev, Kemler \& Begerow, Mycobiota 10: 28 (2020)

Kaseifertia Réblová, Hern.-Restr. \& J. Fourn., Stud. Mycol. 95: 454 (2020)

Keithomyces Samson, Luangsa-ard \& Houbraken, Stud. Mycol. 95: 186 (2020)

Khoyollomyces Hanafy, Lanjekar, Dhakephalkar, T.M. Callaghan, Dagar, G.W. Griff., Elshahed \& N.H. Youssef, Mycologia 112 (6): 1234 (2020)

Knightiellastrum L. Ludw. \& Kantvilas, Lichenologist 52 (3): 217 (2020)

Kosmimatomyces Bianchin., ReinosoFuentealba, Rodr.-Andr., Cano \& Stchigel, Persoonia 44: 403 (2020)

Kukwaea Suija, Motiej. \& Zhurb., Phytotaxa 459 (1): 41 (2020)

Kwanghwana Karun., C.H. Kuo \& K.D. Hyde, Cryptog. Mycol. 41 (6): 124 (2020)

Lacrima Bungartz, Arup \& Søchting, Plant and Fungal Systematics 65 (2): 534 (2020)

Legaliana Van Vooren, Ascomycete.org 12 (4): 188 (2020)

Lendemeriella S.Y. Kondr., Acta bot. hung. 62 (1-2): 116 (2020)

*Liangia H. Yu, Y.B. Wang, Y. Wang, Z.H. Chen \& Zhu L. Yang, Fungal Diversity 103: 22 (2020)

*Ligninsphaeriopsis Phukhams., J.F. Zhang \& K.D. Hyde, Mycosphere 11 (1): 1865 (2020)

Liladisca Baral, Monogr. Orbiliomycetes: 259
(2020)

Lilapila Baral \& G. Marson, Monogr. Orbiliomycetes: 262 (2020)

Linnemannia Vandepol \& Bonito, Fungal Diversity 10.1007/s13225-020-00455-5: 16 (2020)

Linosporopsis Voglmayr \& Beenken, Mycol. Progr. 19 (3): 212 (2020)

Linteromyces Crous, Persoonia 45: 287 (2020)

Longiappendispora Mapook \& K.D. Hyde, Fungal Diversity 101: 139 (2020)

*Longididymella L.W. Hou, L. Cai \& Crous, Stud. Mycol. 96: 339 (2020)

*Longiseptatispora L.W. Hou \& Crous, Fungal Systematics and Evolution 6: 198 (2020)

Longispora Phukhams. \& K.D. Hyde, Fungal Diversity 10.1007/s13225-020-00448-4: 81 (2020)

Longistriata Sulzbacher, Orihara, Grebenc, M.P. Martín \& Baseia, MycoKeys 62: 60 (2020)

Lunasporangiospora Vandepol \& Bonito, Fungal Diversity 10.1007/s13225-02000455-5: 17 (2020)

Lundqvistomyces Y. Marin \& Stchigel, Microorganisms 8 (9, no. 1430): 29 (2020)

*Macroascochyta L.W. Hou, L. Cai \& Crous, Stud. Mycol. 96: 333 (2020)

Macroconstrictolumina Lücking, R. Miranda \& Aptroot, Fungal Diversity 10.1007/s13225-020-00462-6: 168 (2020)

Magnopulchromyces L.B. Conc., Gusmão \& R.F. Castañeda, Fungal Diversity 10.1007/s13225-020-00461-7: 23 (2020)

Malvipezia Van Vooren, Ascomycete.org 12 (4): 188 (2020)

Marantokordyana M. Piepenbr., Maike Hartmann, T.A. Hofm. \& M. Lutz, Mycol. Progr. 19 (4): 357 (2020)

Marquandomyces Samson, Houbraken \& Luangsa-ard, Stud. Mycol. 95: 194 (2020)

*Meniscomyces Q.M. Wang \& F.Y. Bai, Stud. 
Mycol. 96: 113 (2020)

*Mesocorynespora Jian Ma, X.G. Zhang \& R.F. Castañeda, Mycotaxon 135 (3): 618 (2020)

*Microconidiobolus B. Huang \& Y. Nie, MycoKeys 66: 68 (2020)

Mimicoscypha T. Kosonen, Huhtinen \& K. Hansen, Persoonia 46: 49 (2020)

*Mirohelminthosporium K. Zhang, D.W. Li \& R.F. Castañeda, Mycotaxon 135 (4): 778 (2020)

Moringomyces Crous, Persoonia 45: 323 (2020)

Murichromolaenicola Mapook \& K.D. Hyde, Fungal Diversity 101: 71 (2020)

Murinectria M. Niranjan \& V.V. Sarma, Mycosphere 11 (1): 738 (2020)

Muriphila Jurjević, Čmoková \& Hubka, Persoonia 44: 423 (2020)

Myrmecopterula Leal-Dutra, Dentinger \& G.W. Griff., IMA Fungus 11 (no. 2): 12 (2020)

Namibialina Spjut \& Sérus., MycoKeys 73: 33 (2020)

Narcissea D. Wächt. \& A. Melzer, Mycol. Progr. 19 (11): 1203 (2020)

*Natonodosa Heredia, R.F. Castañeda \& D.W. Li, Mycol. Progr. 19 (1): 17 (2020)

Naviculispora Stchigel, Y. Marín, Cano \& Guarro, Microorganisms 8 (9, no. 1430): 14 (2020)

Necromortierella Vandepol \& Bonito, Fungal Diversity 10.1007/s13225-020-00455-5: 18 (2020)

Neoantennariella Abdollahz. \& Crous, Stud. Mycol. 95: 405 (2020)

Neoasbolisia Abdollahz. \& Crous, Stud. Mycol. 95: 406 (2020)

Neobuelliella Hongsanan \& K.D. Hyde, Fungal Diversity 10.1007/s13225-02000462-6: 29 (2020)

Neobyssosphaeria Wanas., E.B.G. Jones \& K.D. Hyde, Fungal Diversity 10.1007/s13225-020-00448-4: 57 (2020)
Neocalonectria Crous, Persoonia 45: 271 (2020)

*Neoconidiobolus B. Huang \& Y. Nie, MycoKeys 66: 70 (2020)

*Neocryphonectria C.M. Tian, N. Jiang \& Crous, Mycologia 112 (2): 282 (2020)

*Neodermea W.J. Li, D.J. Bhat \& K.D. Hyde, Fungal Diversity 100: 610 (2020)

Neodothiora Crous, G.C. Adams \& Winton, Persoonia 45: 325 (2020)

*Neofusicladium Crous, M. Shen \& Y. Zhang ter, Stud. Mycol. 96: 203 (2020)

*Neogloeosporidina W.J. Li, Camporesi \& K.D. Hyde, Fungal Diversity 100: 622 (2020)

*Neohelicascus W. Dong, H. Zhang, K.D. Hyde \& Doilom, Fungal Diversity 10.1007/s13225-020-00463-5: 146 (2020)

*Neojahnula W. Dong, H. Zhang \& K.D. Hyde, Fungal Diversity 10.1007/s13225020-00463-5: 33 (2020)

Neolamproconium Crous \& Akulov, Fungal Systematics and Evolution 6: 204 (2020)

Neoleptosporella Phukhams. \& K.D. Hyde, Fungal Diversity 102: 147 (2020)

*Neomonodictys Y.Z. Lu, C.G. Lin \& K.D. Hyde, Fungal Diversity 100: 207 (2020)

Neoolivea Aime \& McTaggart, Fungal Systematics and Evolution 7: 40 (2020)

Neoophiobolus Mapook \& K.D. Hyde, Fungal Diversity 101: 74 (2020)

Neophaeotheca Abdollahz. \& Crous, Stud. Mycol. 95: 392 (2020)

Neoshiraia H.A. Ariyaw., Scientific Reports 10 (no. 12762): 10 (2020)

Neosorocybe Crous \& Akulov, Fungal Systematics and Evolution 6: 207 (2020)

Neospermospora Crous \& U. Braun, Fungal Systematics and Evolution 7: 84 (2020)

Neostictis Ekanayaka \& K.D. Hyde, Fungal Diversity 10.1007/s13225-020-00448-4: 143 (2020)

Neotorrubiella Tasan., Thanakitp. \& Luangsaard, Persoonia 44: 154 (2020) 
Neoxylaria Konta \& K.D. Hyde, Mycosphere 11 (1): 2636 (2020)

*Nielozyma Xin Zhan Liu, F.Y. Bai, M. Groenew. \& Boekhout, Stud. Mycol. 96: 134 (2020)

Nothoseiridium Crous, Persoonia 44: 323 (2020)

Nothoseptoria Crous \& Bulgakov, Fungal Systematics and Evolution 6: 208 (2020)

Oceanoplaca Arup, Søchting \& Bungartz, Plant and Fungal Systematics 65 (2): 540 (2020)

Ochraceocephala Voglmayr \& Aiello, MycoKeys 66: 12 (2020)

Olegblumia S.Y. Kondr., Lőkös \& Hur, Index Fungorum 466: 1 (2020)

Olotia D. Wächt. \& A. Melzer, Mycol. Progr. 19 (11): 1234 (2020)

Opeltiella S.Y. Kondr., Acta bot. hung. 62 (34): 302 (2020)

Palmeiromyces D.R.S. Pereira \& A.J.L. Phillips, Phytopath. Mediterr. 59 (2): 360 (2020)

Papiliomyces Luangsa-ard, Samson \& Thanakitp., Stud. Mycol. 95: 240 (2020)

Parachaetomium Mehrabi, Asgari \& Zare, Mycol. Progr. 19 (12): 1422 (2020)

*Parafusicladium Crous, M. Shen \& Y. Zhang ter, Stud. Mycol. 96: 206 (2020)

Paragalactinia Van Vooren, Ascomycete.org 12 (4): 188 (2020)

Paraleptospora Mapook \& K.D. Hyde, Fungal Diversity 101: 75 (2020)

Paraloratospora Bundhun, Tennakoon, Phookamsak \& K.D. Hyde, Fungal Diversity 100: 101 (2020)

Paralulworthia A. Poli, E. Bovio, L. Ranieri, G.C. Varese \& V. Prigione, Frontiers in Microbiology 11 (no. 933): 6 (2020)

*Paramicrosphaeropsis L.W. Hou, L. Cai \& Crous, Stud. Mycol. 96: 376 (2020)

*Paramonodictys N.G. Liu, K.D. Hyde \& J.K. Liu, Fungal Diversity 100: 90 (2020)
Paramycetinis R.H. Petersen, Mycotaxon 135: 9 (2020)

Parasulcatispora Phukhams. \& K.D. Hyde, Fungal Diversity 10.1007/s13225-02000448-4: 119 (2020)

Parathyridariella Prigione, A. Poli, E. Bovio \& Varese, Diversity 12 (4, no. 144): 8 (2020)

*Parvomorbus Wen Wang \& S.F. Chen, Persoonia 45: 121 (2020)

Periplasma W.W. Martin \& A. Warren, Mycologia 112 (5): 998 (2020)

Petchia Thanakitp., Mongkols. \& Luangsa-ard, Persoonia 44: 154 (2020)

Phaeoplaca Søchting, Arup \& Bungartz, Plant and Fungal Systematics 65 (2): 546 (2020)

*Phialolunulospora Z.F. Yu \& R.F. Castañeda, MycoKeys 76: 23 (2020)

*Phormopsora Elvebakk, S.G. Hong \& C.H. Park, Mycol. Progr. 19 (11): 1359 (2020)

Phycophthorum Hassett, J. Eukary. Microbiol. 67: 482 (2020)

Phyllocraterina Sérus. \& Aptroot, Fungal Diversity 10.1007/s13225-020-00462-6: 133 (2020)

Phylloscypha Van Vooren, Ascomycete.org 12 (4): 189 (2020)

*Pinaceicola Crous, M. Shen \& Y. Zhang ter, Stud. Mycol. 96: 208 (2020)

Pisutiella S.Y. Kondr., Lőkös \& Farkas, Acta bot. hung. 62 (1-2): 118 (2020)

Podila Stajich, Vandepol \& Bonito, Fungal Diversity 10.1007/s13225-020-00455-5: 18 (2020)

*Protocandelariella Poelt, D. Liu, Hur \& S.Y. Kondr., Acta bot. hung. 62 (3-4): 303 (2020)

Protographum Le Renard, Upchurch, Stockey \& Berbee, Mycologia 112 (3): 511 (2020)

Pruniphilomyces Crous \& Bulgakov, Fungal Systematics and Evolution 6: 215 (2020)

*Pseudobactrodesmium H. Zhang, W. Dong \& K.D. Hyde, Frontiers in Microbiology 11 (no. 565): 3 (2020)

Pseudobogoriella Lücking, R. Miranda \& 
Aptroot, Fungal Diversity 10.1007/s13225020-00462-6: 171 (2020)

Pseudocapulatispora Mapook \& K.D. Hyde, Fungal Diversity 101: 47 (2020)

*Pseudoconlarium N.G. Liu, K.D. Hyde \& J.K. Liu, Fungal Diversity 100: 191 (2020)

Pseudoechria Y. Marín \& Stchigel, Microorganisms 8 (9, no. 1430): 29 (2020)

Pseudohamigera Houbraken, Frisvad \& Samson, Stud. Mycol. 95: 19 (2020)

Pseudohelminthosporium Phukhams. \& K.D. Hyde, Fungal Diversity 10.1007/s13225020-00448-4: 59 (2020)

*Pseudojahnula W. Dong, H. Zhang \& K.D. Hyde, Fungal Diversity 10.1007/s13225020-00463-5: 34 (2020)

*Pseudoleucosporidium V. de García, M.A. Coelho, T. Maia, L.H. Rosa, A.M. Vaz, C.A. Rosa, J.P. Samp., P. Gonç., M.R. van Broock \& Libkind ex M. Groenew. \& Q.M. Wang, Stud. Mycol. 96: 136 (2020)

Pseudomarasmius R.H. Petersen \& K.W. Hughes, Mycotaxon 135: 28 (2020)

Pseudomassarina Phukhams. \& K.D. Hyde, Fungal Diversity 10.1007/s13225-02000448-4: 101 (2020)

*Pseudomicrothyrium X.Y. Zeng, Hongsanan \& K.D. Hyde, Fungal Diversity 10.1007/s13225-020-00462-6: 79 (2020)

Pseudomurilentithecium Mapook \& K.D. Hyde, Fungal Diversity 100: 69 (2020)

Pseudopalawania Mapook \& K.D. Hyde, Biomolecules 10 (no. 569): 10 (2020)

*Pseudopeyronellaea L.W. Hou, L. Cai \& Crous, Stud. Mycol. 96: 339 (2020)

Pseudoroussoella Mapook \& K.D. Hyde, Fungal Diversity 101: 88 (2020)

Pseudoschizothecium Y. Marín, A.N. Mill. \& Stchigel, Microorganisms 8 (9, no. 1430): 32 (2020)

Pseudostaurosphaeria Mapook \& K.D. Hyde, Fungal Diversity 101: 81 (2020)

*Pseudosterigmatospora Q.M. Wang \& F.Y.
Bai, Stud. Mycol. 96: 111 (2020)

Pseudothyridariella Mapook \& K.D. Hyde, Fungal Diversity 101: 98 (2020)

Pseudozeugandromyces De Kesel \& Haelew., Sydowia 72: 291 (2020)

Pulverulina Matheny \& K.W. Hughes, Southeastern Naturalist 19 (3): 452 (2020)

Punjabia D. Wächt. \& A. Melzer, Mycol. Progr. 19 (11): 1235 (2020)

*Purpureofaciens W. Dong, H. Zhang \& K.D. Hyde, Fungal Diversity 10.1007/s13225020-00463-5: 55 (2020)

Purpureomyces Luangsa-ard, Samson \& Thanakitp., Stud. Mycol. 95: 241 (2020)

*Pygmaeomyces E. Walsh \& N. Zhang, Mycologia

10.1080/00275514.2020.1803649: 8 (2020)

*Pyrrhulomyces E.J. Tian \& Matheny, Mycologia

10.1080/00275514.2020.1816067: 9 (2020)

Quaeritorhiza Longcore, D.R. Simmons \& T.Y. James, Mycologia 112 (3): 611 (2020)

Rajchenbergia Salvador-Montoya, Popoff \& Drechsler-Santos, Pl. Syst. Evol. 306 (no. 34): 22 (2020)

Ramiphialis F.R. Barbosa, Fiúza \& R.F. Castañeda, Mycotaxon 135 (2): 294 (2020)

Resinoscypha T. Kosonen, Huhtinen \& K. Hansen, Persoonia 46: 58 (2020)

Rhagadodidymellopsis Fern.-Brime, Gaya, Llimona \& Nav.-Ros., Plant and Fungal Systematics 65: 177 (2020)

*Rhomboidia C.L. Zhao, Mycotaxon 134 (4): 656 (2020)

Rhynchobrunnera B.A. McDonald, U. Braun $\&$ Crous, Fungal Systematics and Evolution 7: 85 (2020)

Rhypophila Y. Marín, A.N. Mill. \& Guarro, Microorganisms 8 (9, no. 1430): 16 (2020)

*Robertozyma Q.M. Wang \& F.Y. Bai, Stud. Mycol. 96: 116 (2020)

Rogerpetersonia Aime \& McTaggart, Fungal Systematics and Evolution 7: 30 (2020) 
*Rosettozyma Q.M. Wang \& F.Y. Bai, Stud. Mycol. 96: 117 (2020)

Rossmanomyces Aime \& McTaggart, Fungal Systematics and Evolution 7: 32 (2020)

Sajamaea Flakus, Piątek \& Rodr. Flakus, Mycol. Progr. 19 (1): 6 (2020)

*Sanguinoderma Y.F. Sun, D.H. Costa \& B.K. Cui, Persoonia 44: 224 (2020)

Savitreea Sakpuntoon, Angchuan, Boonmak, Khunnamw., N. Jacques, Grondin, Casareg. \& Srisuk, Int. J. Syst. Evol. Microbiol. 70 (11): 5668 (2020)

Saxiloba Lücking, Moncada \& Viñas, Plant and Fungal Systematics 65 (2): 580 (2020)

Schummia Lücking, R. Miranda \& Aptroot, Fungal Diversity 10.1007/s13225-02000462-6: 174 (2020)

Sclerenchymomyces Phukhams. \& K.D. Hyde, Fungal Diversity 10.1007/s13225-02000448-4: 41 (2020)

*Sclerotiophoma L.W. Hou, L. Cai \& Crous, Stud. Mycol. 96: 350 (2020)

Serusiauxia Ertz \& Diederich, Plant and Fungal Systematics 65 (1): 61 (2020)

*Serusiauxiella S.H. Jiang, Lücking \& J.C. Wei, Fungal Diversity 102: 274 (2020)

Setoapiospora Mapook \& K.D. Hyde, Fungal Diversity 100: 134 (2020)

Setoarthopyrenia Mapook \& K.D. Hyde, Fungal Diversity 101: 92 (2020)

Similitrichoconis R.F. Castañeda, M. Vera \& D. Sosa, Mycotaxon 135 (4): 830 (2020)

Siphulopsis Kantvilas \& A.R. Nilsen, Lichenologist 52 (3): 218 (2020)

*Solomyces Zhi Y. Zhang, Y.F. Han \& Z.Q. Liang, Frontiers in Microbiology 11 (no. 572596): 11 (2020)

Srinivasanomyces S. Rana \& S.K. Singh, Fungal Diversity 100: 156 (2020)

*Sterigmatospora Q.M. Wang \& F.Y. Bai, Stud. Mycol. 96: 111 (2020)

*Sterila Crous, M. Shen \& Y. Zhang ter, Stud. Mycol. 96: 212 (2020)
*Submersispora W. Dong, H. Zhang \& K.D. Hyde, Fungal Diversity 10.1007/s13225020-00463-5: 122 (2020)

Sucioplaca Bungartz, Søchting \& Arup, Plant and Fungal Systematics 65 (2): 548 (2020)

Sungia Luangsa-ard, Samson \& Thanakitp., Stud. Mycol. 95: 243 (2020)

*Swinscowia S.H. Jiang, Lücking \& Sérus., Fungal Diversity 10.1007/s13225-02000462-6: 136 (2020)

Synnematotriadelphia Chuaseehar., Somrith., Nuankaew \& Boonyuen, Mycol. Progr. 19 (2): 132 (2020)

Tahromyces Hanafy, Lanjekar, Dhakephalkar, T.M. Callaghan, Dagar, G.W. Griff., Elshahed \& N.H. Youssef, Mycologia 112 (6): 1235 (2020)

*Tenuitholiascus S.H. Jiang \& J.C. Wei, IMA Fungus 11 (no. 1): 11 (2020)

*Terrestriporia Y.C. Dai, B.K. Cui, F. Wu, Y. Yuan \& Jia J. Chen, Mycosphere 11 (1): 2761 (2020)

*Teunia Q.M. Wang \& F.Y. Bai, Stud. Mycol. 96: 86 (2020)

*Tricholyophyllum Qing Cai, G. Kost \& Zhu L. Yang, Mycosystema 39 (9): 1734 (2020)

Trichophoma Magaña-Dueñas, Cano \& Stchigel, Persoonia 44: 445 (2020)

Triseptata Boonmee \& Phookamsak, Phytotaxa 447 (4): 257 (2020)

Tulosesus D. Wächt. \& A. Melzer, Mycol. Progr. 19 (11): 1204 (2020)

Tylocliostomum van den Boom \& Magain, Plant and Fungal Systematics 65 (1): 173 (2020)

Vanderaaea Crous, Fungal Systematics and Evolution 7: 94 (2020)

*Vandijckomycella Hern.-Restr., L.W. Hou, L. Cai \& Crous, MycoKeys 65: 86 (2020)

*Varioseptispora L. Qiu, Jian Ma, R.F. Castañeda \& X.G. Zhang, Mycotaxon 135 (4): 754 (2020)

Vesiculozygosporium Crous, Fungal 
Systematics and Evolution 6: 227 (2020)

Vinositunica Koh. Yamam., Degawa \& A. Yamada, Mycologia 112 (2): 319 (2020)

Vredendaliella C.F.J. Spies, Moyo, Halleen \& L. Mostert, Persoonia 45: 206 (2020)

Xenoanthostomella Mapook \& K.D. Hyde, Fungal Diversity 100: 235 (2020)

Xenomonodictys Hern.-Restr., Karimi, Alizadeh \& T. Ghanbary, Persoonia 45: 401 (2020)
Xenoplectosphaerella Jayaward., Phukhams. \& K.D. Hyde, Fungal Diversity 10.1007/s13225-020-00448-4: 176 (2020)

Xenoroussoella Mapook \& K.D. Hyde, Fungal Diversity 101: 93 (2020)

*Yamadamyces Q.M. Wang, F.Y. Bai, M. Groenew. \& Boekhout, Stud. Mycol. 96: 138 (2020)

Yosiokobayasia Samson, Luangsa-ard \& Thanakitp., Stud. Mycol. 95: 243 (2020)

\section{亚属 Subgenera}

*Russula subgen. Glutinosae Buyck \& X.H. Wang, Mycosphere 11 (1): 288 (2020)

Umbilicaria subgen. Papillophora Davydov,
Ahti \& Sennikov, Mycotaxon 135 (1): 139 (2020)

\section{组 Sections}

Aspergillus sect. Bispori S.W. Peterson, Varga, Frisvad, Samson ex Houbraken, Stud. Mycol. 95: 46 (2020)

Aspergillus sect. Polypaecilum Houbraken \& Frisvad, Stud. Mycol. 95: 54 (2020)

Aspergillus sect. Raperorum S.W. Peterson, Varga, Frisvad, Samson ex Houbraken, Stud. Mycol. 95: 52 (2020)

Aspergillus sect. Silvatici S.W. Peterson, Varga, Frisvad, Samson ex Houbraken, Stud. Mycol. 95: 52 (2020)

Aspergillus sect. Vargarum Houbraken \& Frisvad, Stud. Mycol. 95: 46 (2020)

*Clitopilus sect. Crispi S.P. Jian \& Zhu L. Yang, Mycologia 112 (2): 385 (2020)

Coprinellus sect. Aureogranulati D. Wächt. \& A. Melzer, Mycol. Progr. 19 (11): 1194 (2020)

Coprinellus sect. Curti D. Wächt. \& A. Melzer, Mycol. Progr. 19 (11): 1198 (2020)

Coprinellus sect. Deminuti D. Wächt. \& A. Melzer, Mycol. Progr. 19 (11): 1202 (2020)

Coprinellus sect. Disseminati D. Wächt. \& A. Melzer, Mycol. Progr. 19 (11): 1192 (2020)
Coprinellus sect. Hepthemeri D. Wächt. \& A. Melzer, Mycol. Progr. 19 (11): 1201 (2020)

Coprinopsis sect. Alopeciae D. Wächt. \& A. Melzer, Mycol. Progr. 19 (11): 1244 (2020)

Coprinopsis sect. Canocipes D. Wächt. \& A. Melzer, Mycol. Progr. 19 (11): 1255 (2020)

Coprinopsis sect. Cinereae D. Wächt. \& A. Melzer, Mycol. Progr. 19 (11): 1238 (2020)

Coprinopsis sect. Erythrocephalae D. Wächt. \& A. Melzer, Mycol. Progr. 19 (11): 1250 (2020)

Coprinopsis sect. Filamentiferae D. Wächt. \& A. Melzer, Mycol. Progr. 19 (11): 1253 (2020)

Coprinopsis sect. Geesteranorum D. Wächt. \& A. Melzer, Mycol. Progr. 19 (11): 1245 (2020)

Coprinopsis sect. Krieglsteinerorum D. Wächt. \& A. Melzer, Mycol. Progr. 19 (11): 1249 (2020)

Coprinopsis sect. Melanthinae D. Wächt. \& A. Melzer, Mycol. Progr. 19 (11): 1254 (2020)

Coprinopsis sect. Mitrisporae D. Wächt. \& A. Melzer, Mycol. Progr. 19 (11): 1243 (2020) 
Coprinopsis sect. Phlyctidosporae D. Wächt. \& A. Melzer, Mycol. Progr. 19 (11): 1247 (2020)

Coprinopsis sect. Quartoconatae D. Wächt. \& A. Melzer, Mycol. Progr. 19 (11): 1255 (2020)

Coprinopsis sect. Radiatae D. Wächt. \& A. Melzer, Mycol. Progr. 19 (11): 1253 (2020)

Coprinopsis sect. Subniveae D. Wächt. \& A. Melzer, Mycol. Progr. 19 (11): 1254 (2020)

Coprinopsis sect. Xenobiae D. Wächt. \& A. Melzer, Mycol. Progr. 19 (11): 1244 (2020)

Cortinarius sect. Alboambiti Niskanen, Liimat. \& Ammirati, Index Fungorum 439: 11 (2020)

Cortinarius sect. Alnicolarum Kytöv., Niskanen \& Liimat., Index Fungorum 439: 3 (2020)

Cortinarius sect. Atroalbi Niskanen, Kytöv. \& Liimat., Index Fungorum 439: 4 (2020)

Cortinarius sect. Bibuli Niskanen, Kytöv. \& Liimat., Index Fungorum 439: 6 (2020)

Cortinarius sect. Bombycini Ammirati, Niskanen \& Liimat., Index Fungorum 439: 6 (2020)

Cortinarius sect. Cacaodisci Kytöv., Niskanen \& Liimat., Index Fungorum 439: 9 (2020)

Cortinarius sect. Caliginosi Liimat., Niskanen \& Kytöv., Index Fungorum 439: 4 (2020)

Cortinarius sect. Cisqhale Liimat., Niskanen \& Ammirati, Index Fungorum 439: 7 (2020)

Cortinarius sect. Crassispori Kytöv., Niskanen \& Liimat., Index Fungorum 439: 6 (2020)

Cortinarius sect. Craticii Niskanen, Kytöv. \& Liimat., Index Fungorum 439: 10 (2020)

Cortinarius sect. Duristipedes Kytöv., Niskanen \& Liimat., Index Fungorum 439: 4 (2020)

Cortinarius sect. Exsulares Ammirati, Liimat. \& Niskanen, Index Fungorum 439: 7 (2020)

Cortinarius sect. Flexibilifolii Liimat., Niskanen \& Kytöv., Index Fungorum 439: 2 (2020)
Cortinarius sect. Flexipedes Kytöv., Niskanen \& Liimat., Index Fungorum 439: 1 (2020)

Cortinarius sect. Friesiorum Liimat., Kytöv. \& Niskanen, Index Fungorum 439: 5 (2020)

Cortinarius sect. Furvi Liimat., Kytöv. \& Niskanen, Index Fungorum 439: 7 (2020)

Cortinarius sect. Heterospori Kytöv., Niskanen \& Liimat., Index Fungorum 439: 1 (2020)

Cortinarius sect. Incisiores Liimat., Niskanen \& Kytöv., Index Fungorum 439: 2 (2020)

Cortinarius sect. Ionosmi Niskanen, Kytöv. \& Liimat., Index Fungorum 439: 10 (2020)

Cortinarius sect. Leiocastanei Niskanen, Liimat. \& Kytöv., Index Fungorum 439: 2 (2020)

Cortinarius sect. Megaspori Kytöv., Niskanen \& Liimat., Index Fungorum 439: 4 (2020)

Cortinarius sect. Micro-ornati Niskanen, Kytöv. \& Liimat., Index Fungorum 439: 9 (2020)

Cortinarius sect. Nucicolores Niskanen, Kytöv. \& Liimat., Index Fungorum 439: 3 (2020)

Cortinarius sect. Ochropallescentes Liimat., Niskanen \& Ammirati, Index Fungorum 439: 11 (2020)

Cortinarius sect. Ochrovelati Niskanen, Kytöv. \& Liimat., Index Fungorum 439: 1 (2020)

Cortinarius sect. Olorinati Soop \& Dima, Phytotaxa 438 (4): 232 (2020)

Cortinarius sect. Phaeosmi Liimat., Niskanen \& Kytöv., Index Fungorum 439: 8 (2020)

Cortinarius sect. Politi Ammirati, Niskanen \& Liimat., Index Fungorum 439: 6 (2020)

Cortinarius sect. Praestigiosi Kytöv., Niskanen \& Liimat., Index Fungorum 439: 5 (2020)

Cortinarius sect. Privignati Niskanen, Kytöv. \& Liimat., Index Fungorum 439: 11 (2020)

Cortinarius sect. Pseudobicolores Liimat., Niskanen \& Kytöv., Index Fungorum 439: 8 (2020) 
Cortinarius sect. Pseudoduracini Liimat., Niskanen \& Kytöv., Index Fungorum 439: 3 (2020)

Cortinarius sect. Pseudotragani Niskanen, Liimat. \& Ammirati, Index Fungorum 439: 10 (2020)

Cortinarius sect. Punctatiformes Liimat., Niskanen \& Kytöv., Index Fungorum 439: 5 (2020)

Cortinarius sect. Ravi Liimat., Kytöv., Niskanen \& Ammirati, Index Fungorum 439: 3 (2020)

Cortinarius sect. Rubrocincti Liimat., Niskanen \& Kytöv., Index Fungorum 439: 2 (2020)

Cortinarius sect. Rustici Niskanen, Kytöv. \& Liimat., Index Fungorum 439: 7 (2020)

Cortinarius sect. Sejunctifolii Liimat., Niskanen \& Kytöv., Index Fungorum 439: 9 (2020)

Cortinarius sect. Sporagniti Kytöv., Niskanen \& Liimat., Index Fungorum 439: 1 (2020)

Cortinarius sect. Squalidi Liimat., Ammirati \& Niskanen, Index Fungorum 439: 6 (2020)

Cortinarius sect. Tortuosi Niskanen, Kytöv. \& Liimat., Index Fungorum 439: 9 (2020)

Cortinarius sect. Tragani Niskanen, Kytöv. \& Liimat., Index Fungorum 439: 8 (2020)

Cortinarius sect. Valgi Niskanen, Kytöv. \& Liimat., Index Fungorum 439: 10 (2020)

Cortinarius sect. Verni Kytöv., Niskanen \& Liimat., Index Fungorum 439: 3 (2020)

Cortinarius sect. Viliores Liimat., Niskanen \& Kytöv., Index Fungorum 439: 9 (2020)

Cortinarius sect. Vinaceobrunnei Ammirati, Niskanen \& Liimat., Index Fungorum 439: 5 (2020)

Cortinarius subsect. Varii Soop, Brandrud, Saar \& Dima, Journal des JEC 22: 37 (2020)

Hebeloma sect. Adherentia Monedero \& P. Alvarado, Yesca: 60 (2020)

Lactifluus sect. Nebulosi Delgat, Persoonia 44: 286 (2020)
Lactifluus sect. Neotropici J. Duque, Delgat, Verbeken, M.A. Neves \& A.A. Carvalho, Syst. Biodiv. 18 (4): 351 (2020)

Leccinellum sect. Calidorum C. Hahn, Mycol. bavarica 20: 123 (2020)

Leccinellum sect. Hemixantha C. Hahn, Mycol. bavarica 20: 123 (2020)

Orbilia sect. Aurantiorubrae Baral \& E. Weber, Monogr. Orbiliomycetes: 769 (2020)

Orbilia sect. Lentiformes Baral \& E. Weber, Monogr. Orbiliomycetes: 457 (2020)

Orbilia sect. Ovoideae Baral \& E. Weber, Monogr. Orbiliomycetes: 1241 (2020)

Parasola sect. Conopileae D. Wächt. \& A. Melzer, Mycol. Progr. 19 (11): 1257 (2020)

Penicillium sect. Alfrediorum Houbraken \& Frisvad, Stud. Mycol. 95: 55 (2020)

Penicillium sect. Crypta Houbraken \& Frisvad, Stud. Mycol. 95: 69 (2020)

Penicillium sect. Eremophila Houbraken \& Frisvad, Stud. Mycol. 95: 69 (2020)

Penicillium sect. Formosana Houbraken \& Frisvad, Stud. Mycol. 95: 82 (2020)

Penicillium sect. Griseola Houbraken \& Frisvad, Stud. Mycol. 95: 73 (2020)

Penicillium sect. Inusitata Houbraken \& Frisvad, Stud. Mycol. 95: 72 (2020)

Penicillium sect. Lasseniorum Houbraken \& Frisvad, Stud. Mycol. 95: 75 (2020)

Psathyrella sect. Arenosae D. Wächt. \& A. Melzer, Mycol. Progr. 19 (11): 1221 (2020)

Psathyrella sect. Confusae D. Wächt. \& A. Melzer, Mycol. Progr. 19 (11): 1221 (2020)

Psathyrella sect. Jacobssoniorum D. Wächt. \& A. Melzer, Mycol. Progr. 19 (11): 1223 (2020)

Psathyrella sect. Noli-tangere D. Wächt. \& A. Melzer, Mycol. Progr. 19 (11): 1217 (2020)

Psathyrella sect. Saponaceae D. Wächt. \& A. Melzer, Mycol. Progr. 19 (11): 1220 (2020) Psathyrella sect. Sinefibularum D. Wächt. \& A. Melzer, Mycol. Progr. 19 (11): 1228 (2020) 
Psathyrella sect. Stridvalliorum D. Wächt. \&

A. Melzer, Mycol. Progr. 19 (11): 1221 (2020)
*Talaromyces sect. Tenues B.D. Sun, A.J. Chen, Houbraken \& Samson, MycoKeys 68: $82(2020)$

\section{亚组 Subsections}

Cortinarius subsect. Carabi Kytöv., Niskanen \& Liimat., Index Fungorum 439: 12 (2020)

Cortinarius subsect. Flavobasiles Ammirati, Niskanen \& Liimat., Index Fungorum 439: 8 (2020)

Cortinarius subsect. Furvolaesi Niskanen, Kytöv. \& Liimat., Index Fungorum 439: 12 (2020)

Cortinarius subsect. Terribiles Niskanen, Liimat. \& Kytöv., Index Fungorum 439: 11
(2020)

Marasmius subsect. Globulares J.S. Oliveira \& Moncalvo, Persoonia 44: 274 (2020)

Marasmius subsect. Leonini J.S. Oliveira \& Moncalvo, Persoonia 44: 272 (2020)

Marasmius subsect. Spinulosi J.S. Oliveira \& Moncalvo, Persoonia 44: 274 (2020)

*Russula subsect. Castanopsidum Buyck \& X.H. Wang, Cryptog. Mycol. 41 (4): 101 (2020)

\section{系 Series}

Aspergillus ser. Acidohumorum Houbraken \& Frisvad, Stud. Mycol. 95: 42 (2020)

Aspergillus ser. Alliacei Houbraken \& Frisvad, Stud. Mycol. 95: 34 (2020)

Aspergillus ser. Ambigui Houbraken \& Frisvad, Stud. Mycol. 95: 39 (2020)

Aspergillus ser. Arxiorum Houbraken \& Frisvad, Stud. Mycol. 95: 40 (2020)

Aspergillus ser. Aurantiobrunnei Houbraken \& Frisvad, Stud. Mycol. 95: 47 (2020)

Aspergillus ser. Avenacei Houbraken \& Frisvad, Stud. Mycol. 95: 34 (2020)

Aspergillus ser. Bertholletiarum Houbraken \& Frisvad, Stud. Mycol. 95: 35 (2020)

Aspergillus ser. Biplani Houbraken \& Frisvad, Stud. Mycol. 95: 52 (2020)

Aspergillus ser. Brevipedes Houbraken \& Frisvad, Stud. Mycol. 95: 43 (2020)

Aspergillus ser. Brunneouniseriati Houbraken \& Frisvad, Stud. Mycol. 95: 40 (2020)

Aspergillus ser. Calidousti Houbraken \& Frisvad, Stud. Mycol. 95: 53 (2020)

Aspergillus ser. Canini Houbraken \& Frisvad,
Stud. Mycol. 95: 54 (2020)

Aspergillus ser. Carbonarii Houbraken \& Frisvad, Stud. Mycol. 95: 37 (2020)

Aspergillus ser. Cavernicolarum Houbraken \& Frisvad, Stud. Mycol. 95: 47 (2020)

Aspergillus ser. Cervini Houbraken \& Frisvad, Stud. Mycol. 95: 42 (2020)

Aspergillus ser. Chevalierorum Houbraken \& Frisvad, Stud. Mycol. 95: 26 (2020)

Aspergillus ser. Circumdati Houbraken \& Frisvad, Stud. Mycol. 95: 31 (2020)

Aspergillus ser. Conjuncti Houbraken \& Frisvad, Stud. Mycol. 95: 52 (2020)

Aspergillus ser. Coremiiformes Houbraken \& Frisvad, Stud. Mycol. 95: 35 (2020)

Aspergillus ser. Cremei Houbraken \& Frisvad, Stud. Mycol. 95: 40 (2020)

Aspergillus ser. Deflecti Houbraken \& Frisvad, Stud. Mycol. 95: 53 (2020)

Aspergillus ser. Egyptiaci Houbraken \& Frisvad, Stud. Mycol. 95: 47 (2020)

Aspergillus ser. Fennelliarum Houbraken \& Frisvad, Stud. Mycol. 95: 43 (2020)

Aspergillus ser. Flavi Houbraken \& Frisvad, 
Stud. Mycol. 95: 35 (2020)

Aspergillus ser. Flavipedes Houbraken \&

Frisvad, Stud. Mycol. 95: 36 (2020)

Aspergillus ser. Fumigati Houbraken \& Frisvad, Stud. Mycol. 95: 43 (2020)

Aspergillus ser. Funiculosi Houbraken \&

Frisvad, Stud. Mycol. 95: 51 (2020)

Aspergillus ser. Halophilici Houbraken \& Frisvad, Stud. Mycol. 95: 28 (2020)

Aspergillus ser. Heteromorphi Houbraken \&

Frisvad, Stud. Mycol. 95: 37 (2020)

Aspergillus ser. Homomorphi Houbraken \&

Frisvad, Stud. Mycol. 95: 37 (2020)

Aspergillus ser. Implicati Houbraken \&

Frisvad, Stud. Mycol. 95: 53 (2020)

Aspergillus ser. Japonici Houbraken \&

Frisvad, Stud. Mycol. 95: 38 (2020)

Aspergillus ser. Kalimarum Houbraken \&

Frisvad, Stud. Mycol. 95: 54 (2020)

Aspergillus ser. Kitamyces Houbraken \&

Frisvad, Stud. Mycol. 95: 35 (2020)

Aspergillus ser. Leporum Houbraken \&

Frisvad, Stud. Mycol. 95: 35 (2020)

Aspergillus ser. Leucocarpi Houbraken \&

Frisvad, Stud. Mycol. 95: 27 (2020)

Aspergillus ser. Monodiorum Houbraken \&

Frisvad, Stud. Mycol. 95: 53 (2020)

Aspergillus ser. Multicolores Houbraken \&

Frisvad, Stud. Mycol. 95: 47 (2020)

Aspergillus ser. Neoglabri Houbraken \&

Frisvad, Stud. Mycol. 95: 43 (2020)

Aspergillus ser. Neonivei Houbraken \&

Frisvad, Stud. Mycol. 95: 36 (2020)

Aspergillus ser. Nidulantes Houbraken \&

Frisvad, Stud. Mycol. 95: 50 (2020)

Aspergillus ser. Nigri Houbraken \& Frisvad,

Stud. Mycol. 95: 38 (2020)

Aspergillus ser. Nivei Houbraken \& Frisvad,

Stud. Mycol. 95: 40 (2020)

Aspergillus ser. Nomiarum Houbraken \&

Frisvad, Stud. Mycol. 95: 36 (2020)

Aspergillus ser. Noonimiarum Houbraken \&

Frisvad, Stud. Mycol. 95: 54 (2020)
Aspergillus ser. Ochraceorosei Houbraken \& Frisvad, Stud. Mycol. 95: 51 (2020)

Aspergillus ser. Olivimuriarum Houbraken \& Frisvad, Stud. Mycol. 95: 36 (2020)

Aspergillus ser. Penicillioides Houbraken \& Frisvad, Stud. Mycol. 95: 29 (2020)

Aspergillus ser. Polypaecilum Houbraken \& Frisvad, Stud. Mycol. 95: 55 (2020)

Aspergillus ser. Pulvini Houbraken \& Frisvad,

Stud. Mycol. 95: 41 (2020)

Aspergillus ser. Restricti Houbraken \& Frisvad, Stud. Mycol. 95: 31 (2020)

Aspergillus ser. Rubri Houbraken \& Frisvad, Stud. Mycol. 95: 27 (2020)

Aspergillus ser. Salinarum Houbraken \& Frisvad, Stud. Mycol. 95: 55 (2020)

Aspergillus ser. Sclerotiorum Houbraken \& Frisvad, Stud. Mycol. 95: 34 (2020)

Aspergillus ser. Sparsi Houbraken \& Frisvad, Stud. Mycol. 95: 53 (2020)

Aspergillus ser. Spathulati Houbraken \& Frisvad, Stud. Mycol. 95: 43 (2020)

Aspergillus ser. Spelaei Houbraken \& Frisvad, Stud. Mycol. 95: 36 (2020)

Aspergillus ser. Speluncei Houbraken \& Frisvad, Stud. Mycol. 95: 50 (2020)

Aspergillus ser. Stellati Houbraken \& Frisvad, Stud. Mycol. 95: 50 (2020)

Aspergillus ser. Steyniorum Houbraken \& Frisvad, Stud. Mycol. 95: 34 (2020)

Aspergillus ser. Tamarindosolorum Houbraken \& Frisvad, Stud. Mycol. 95: 27 (2020)

Aspergillus ser. Teporium Houbraken \& Frisvad, Stud. Mycol. 95: 27 (2020)

Aspergillus ser. Terrei Houbraken \& Frisvad, Stud. Mycol. 95: 40 (2020)

Aspergillus ser. Thermomutati Houbraken \& Frisvad, Stud. Mycol. 95: 45 (2020)

Aspergillus ser. Unguium Houbraken \& Frisvad, Stud. Mycol. 95: 51 (2020)

Aspergillus ser. Unilaterales Houbraken \& Frisvad, Stud. Mycol. 95: 45 (2020) 
Aspergillus ser. Usti Houbraken \& Frisvad, Stud. Mycol. 95: 54 (2020)

Aspergillus ser. Versicolores Houbraken \& Frisvad, Stud. Mycol. 95: 51 (2020)

Aspergillus ser. Viridinutantes Houbraken \& Frisvad, Stud. Mycol. 95: 45 (2020)

Aspergillus ser. Vitricolarum Houbraken \& Frisvad, Stud. Mycol. 95: 31 (2020)

Aspergillus ser. Wentiorum Houbraken \& Frisvad, Stud. Mycol. 95: 41 (2020)

Aspergillus ser. Whitfieldiorum Houbraken \& Frisvad, Stud. Mycol. 95: 55 (2020)

Aspergillus ser. Xerophili Houbraken \& Frisvad, Stud. Mycol. 95: 27 (2020)

Marasmius ser. Brunneospermi J.S. Oliveira \& Moncalvo, Persoonia 44: 275 (2020)

Marasmius ser. Cohaerentes J.S. Oliveira \& Moncalvo, Persoonia 44: 274 (2020)

Marasmius ser. Conferti J.S. Oliveira \& Moncalvo, Persoonia 44: 273 (2020)

Marasmius ser. Corrugati J.S. Oliveira \& Moncalvo, Persoonia 44: 272 (2020)

Marasmius ser. Crinipes J.S. Oliveira \& Moncalvo, Persoonia 44: 274 (2020)

Marasmius ser. Graminicolae J.S. Oliveira \& Moncalvo, Persoonia 44: 272 (2020)

Marasmius ser. Imitarii J.S. Oliveira \& Moncalvo, Persoonia 44: 274 (2020)

Marasmius ser. Luteoli J.S. Oliveira \& Moncalvo, Persoonia 44: 273 (2020)

Marasmius ser. Pulcherripes J.S. Oliveira \& Moncalvo, Persoonia 44: 274 (2020)

Marasmius ser. Purpureostriati J.S. Oliveira \& Moncalvo, Persoonia 44: 273 (2020)

Marasmius ser. Rhabarbarini J.S. Oliveira \& Moncalvo, Persoonia 44: 273 (2020)

Marasmius ser. Silvicolae J.S. Oliveira \& Moncalvo, Persoonia 44: 275 (2020)

Marasmius ser. Wynnearum J.S. Oliveira \& Moncalvo, Persoonia 44: 275 (2020)

Orbilia ser. Abutilones Baral \& E. Weber, Monogr. Orbiliomycetes: 885 (2020)

Orbilia ser. Albovinosae Baral, Monogr.
Orbiliomycetes: 932 (2020)

Orbilia ser. Cercidicola Baral, Monogr. Orbiliomycetes: 501 (2020)

Orbilia ser. Commatoideae Baral \& E. Weber, Monogr. Orbiliomycetes: 802 (2020)

Orbilia ser. Ellipsospermae Baral \& E. Weber, Monogr. Orbiliomycetes: 1170 (2020)

Orbilia ser. Hesperideae Baral \& E. Weber, Monogr. Orbiliomycetes: 551 (2020)

Orbilia ser. Hyalinia Baral \& E. Weber, Monogr. Orbiliomycetes: 868 (2020)

Orbilia ser. Microsomates Baral \& E. Weber, Monogr. Orbiliomycetes: 1295 (2020)

Orbilia ser. Microspermae Baral \& E. Weber, Monogr. Orbiliomycetes: 528 (2020)

Orbilia ser. Neodactylella Baral \& E. Weber, Monogr. Orbiliomycetes: 1437 (2020)

Orbilia ser. Ovales Baral \& E. Weber, Monogr. Orbiliomycetes: 498 (2020)

Orbilia ser. Phanosomates Baral \& E. Weber, Monogr. Orbiliomycetes: 505 (2020)

Orbilia ser. Piliferae Baral, Monogr. Orbiliomycetes: 915 (2020)

Orbilia ser. Poitevinicae Baral, Monogr. Orbiliomycetes: 913 (2020)

Orbilia ser. Regales Baral \& E. Weber, Monogr. Orbiliomycetes: 773 (2020)

Orbilia ser. Rubellae Baral \& E. Weber, Monogr. Orbiliomycetes: 820 (2020)

Orbilia ser. Serpentinae Baral \& E. Weber, Monogr. Orbiliomycetes: 1049 (2020)

Orbilia ser. Vibrioides Baral, E. Weber \& G. Marson, Monogr. Orbiliomycetes: 746 (2020)

Orbilia ser. Xanthoguttulatae Baral, Quijada \& E. Weber, Monogr. Orbiliomycetes: 845 (2020)

Penicillium ser. Adametziorum Houbraken \& Frisvad, Stud. Mycol. 95: 76 (2020)

Penicillium ser. Angustiporcata Houbraken \& Frisvad, Stud. Mycol. 95: 72 (2020)

Penicillium ser. Atramentosa Houbraken \& Frisvad, Stud. Mycol. 95: 82 (2020) 
Penicillium ser. Brevicompacta Houbraken \&

Frisvad, Stud. Mycol. 95: 78 (2020)

Penicillium ser. Buchwaldiorum Houbraken \& Frisvad, Stud. Mycol. 95: 78 (2020)

Penicillium ser. Cinnamopurpurea Houbraken \& Frisvad, Stud. Mycol. 95: 60 (2020)

Penicillium ser. Clavigera Houbraken \& Frisvad, Stud. Mycol. 95: 83 (2020)

Penicillium ser. Copticolarum Houbraken \& Frisvad, Stud. Mycol. 95: 61 (2020)

Penicillium ser. Corylophila Houbraken \& Frisvad, Stud. Mycol. 95: 70 (2020)

Penicillium ser. Costaricensia Houbraken \& Frisvad, Stud. Mycol. 95: 59 (2020)

Penicillium ser. Dalearum Houbraken \& Frisvad, Stud. Mycol. 95: 73 (2020)

Penicillium ser. Estinogena Houbraken \& Frisvad, Stud. Mycol. 95: 72 (2020)

Penicillium ser. Euglauca Houbraken \& Frisvad, Stud. Mycol. 95: 61 (2020)

Penicillium ser. Fortuita Houbraken \& Frisvad, Stud. Mycol. 95: 56 (2020)

Penicillium ser. Gallaica Houbraken \& Frisvad, Stud. Mycol. 95: 68 (2020)

Penicillium ser. Georgiensia Houbraken \& Frisvad, Stud. Mycol. 95: 76 (2020)

Penicillium ser. Glandicolarum Houbraken \& Frisvad, Stud. Mycol. 95: 85 (2020)

Penicillium ser. Goetziorum Houbraken \& Frisvad, Stud. Mycol. 95: 80 (2020)

Penicillium ser. Gracilenta Houbraken \& Frisvad, Stud. Mycol. 95: 72 (2020)

Penicillium ser. Herqueorum Houbraken \& Frisvad, Stud. Mycol. 95: 76 (2020)

Penicillium ser. Hoeksiorum Houbraken \& Frisvad, Stud. Mycol. 95: 56 (2020)

Penicillium ser. Idahoensia Houbraken \& Frisvad, Stud. Mycol. 95: 60 (2020)

Penicillium ser. Improvisa Houbraken \& Frisvad, Stud. Mycol. 95: 56 (2020)

Penicillium ser. Indica Houbraken \& Frisvad, Stud. Mycol. 95: 60 (2020)
Penicillium ser. Jiangxiensia Houbraken \& Frisvad, Stud. Mycol. 95: 61 (2020)

Penicillium ser. Kiamaensia Houbraken \& Frisvad, Stud. Mycol. 95: 56 (2020)

Penicillium ser. Livida Houbraken \& Frisvad, Stud. Mycol. 95: 57 (2020)

Penicillium ser. Longicatenata Houbraken \& Frisvad, Stud. Mycol. 95: 57 (2020)

Penicillium ser. Macrosclerotiorum Houbraken \& Frisvad, Stud. Mycol. 95: 72 (2020)

Penicillium ser. Nodula Houbraken \& Frisvad, Stud. Mycol. 95: 61 (2020)

Penicillium ser. Osmophila Houbraken \& Frisvad, Stud. Mycol. 95: 82 (2020)

Penicillium ser. Paradoxa Houbraken \& Frisvad, Stud. Mycol. 95: 82 (2020)

Penicillium ser. Paxillorum Houbraken \& Frisvad, Stud. Mycol. 95: 68 (2020)

Penicillium ser. Phoenicea Houbraken \& Frisvad, Stud. Mycol. 95: 60 (2020)

Penicillium ser. Quercetorum Houbraken \& Frisvad, Stud. Mycol. 95: 58 (2020)

Penicillium ser. Raistrickiorum Houbraken \& Frisvad, Stud. Mycol. 95: 84 (2020)

Penicillium ser. Ramigena Houbraken \& Frisvad, Stud. Mycol. 95: 76 (2020)

Penicillium ser. Robsamsonia Houbraken \& Frisvad, Stud. Mycol. 95: 85 (2020)

Penicillium ser. Rolfsiorum Houbraken \& Frisvad, Stud. Mycol. 95: 74 (2020)

Penicillium ser. Roseopurpurea Houbraken \& Frisvad, Stud. Mycol. 95: 68 (2020)

Penicillium ser. Samsoniorum Houbraken \& Frisvad, Stud. Mycol. 95: 82 (2020)

Penicillium ser. Saturniformia Houbraken \& Frisvad, Stud. Mycol. 95: 58 (2020)

Penicillium ser. Scabrosa Houbraken \& Frisvad, Stud. Mycol. 95: 84 (2020)

Penicillium ser. Sclerotigena Houbraken \& Frisvad, Stud. Mycol. 95: 84 (2020)

Penicillium ser. Sclerotiorum Houbraken \& Frisvad, Stud. Mycol. 95: 77 (2020) 
Penicillium ser. Sheariorum Houbraken \&

Frisvad, Stud. Mycol. 95: 68 (2020)

Penicillium ser. Simplicissima Houbraken \&

Frisvad, Stud. Mycol. 95: 74 (2020)

Penicillium ser. Soppiorum Houbraken \&

Frisvad, Stud. Mycol. 95: 84 (2020)

Penicillium ser. Spinulosa Houbraken \&

Frisvad, Stud. Mycol. 95: 58 (2020)

Penicillium ser. Sublectatica Houbraken \&

Frisvad, Stud. Mycol. 95: 58 (2020)

Penicillium ser. Sumatraensia Houbraken \&
Frisvad, Stud. Mycol. 95: 68 (2020)

Penicillium ser. Thiersiorum Houbraken \& Frisvad, Stud. Mycol. 95: 58 (2020)

Penicillium ser. Thomiorum Houbraken \& Frisvad, Stud. Mycol. 95: 59 (2020)

Penicillium ser. Verhageniorum Houbraken \& Frisvad, Stud. Mycol. 95: 59 (2020)

Penicillium ser. Virgata Houbraken \& Frisvad, Stud. Mycol. 95: 85 (2020)

Penicillium ser. Westlingiorum Houbraken \& Frisvad, Stud. Mycol. 95: 69 (2020)

\section{种 Species}

Absidia pararepens Jurjević, M. Kolařík \& Hubka, Persoonia 44: 351 (2020)

Acanthothecis normuralis Aptroot, Archive For Lichenology 16: 2 (2020)

Acanthothecis psoromica Aptroot \& Feuerstein, Archive For Lichenology 16: 2 (2020)

Acanthotrema minus Aptroot, Archive For Lichenology 16: 3 (2020)

Acarospora scottii K. Knudsen \& Kocourk., Mycotaxon 135 (1): 455 (2020)

Acervuloseptoria fraxini Crous \& Bulgakov, Fungal Systematics and Evolution 6: 175 (2020)

Achaetomium aegilopis Mehrabi, Asgari \& Zare, Mycol. Progr. 19 (12): 1427 (2020)

Acremonium behniae Crous, Persoonia 45: 317 (2020)

*Acremonium chiangraiense J.F. Li, R.H. Perera \& Phookamsak, Fungal Diversity 100: 197 (2020)

Acrocalymma bipolare Abdel-Aziz \& AbdelWahab, Fungal Diversity 10.1007/s13225020-00463-5: 45 (2020)

*Acrogenospora aquatica D.F. Bao, Z.L. Luo, K.D. Hyde \& H.Y. Su, Frontiers in Microbiology 11 (no. 1606): 3 (2020)

*Acrogenospora basalicellularispora D.F. Bao, Z.L. Luo, K.D. Hyde \& H.Y. Su,
Frontiers in Microbiology 11 (no. 1606): 8 (2020)

*Acrogenospora guttulatispora D.F. Bao, Z.L. Luo, K.D. Hyde \& H.Y. Su, Frontiers in Microbiology 11 (no. 1606): 8 (2020)

*Acrogenospora obovoidispora D.F. Bao, Z.L. Luo, K.D. Hyde \& H.Y. Su, Frontiers in Microbiology 11 (no. 1606): 8 (2020)

*Acrogenospora olivaceospora D.F. Bao, Z.L. Luo, K.D. Hyde \& H.Y. Su, Frontiers in Microbiology 11 (no. 1606): 10 (2020)

*Acrogenospora submersa D.F. Bao, Z.L. Luo, K.D. Hyde \& H.Y. Su, Frontiers in Microbiology 11 (no. 1606): 12 (2020)

*Acrogenospora yunnanensis D.F. Bao, Z.L. Luo, K.D. Hyde \& H.Y. Su, Frontiers in Microbiology 11 (no. 1606): 18 (2020)

Acrospermum urticae D. Pem, Camporesi \& K.D. Hyde, Fungal Diversity 10.1007/s13225-020-00462-6: 16 (2020)

Adustochaete nivea Alvarenga, Fungal Diversity 100: 255 (2020)

Aequabiliella palatina C. Kraus, Damm, S. Bien, Vögele \& M. Fisch., Fungal Systematics and Evolution 6: 145 (2020)

Aestipascuomyces dupliciliberans Stabel, R. Hanafy, Schweitzer, Greif, Aliyu, Flad, D. Young, Lebuhn, Elshahed, Ochsenreither \& N.H. Youssef, Microorganisms 8 (11): 13 
(2020)

*Agaricus albiceps R.L. Zhao \& B. Cao, Mycologia 113 (1): 195 (2020)

*Agaricus alboumbonatus R.L. Zhao \& B. Cao, Mycologia 113 (1): 195 (2020)

*Agaricus catenocystis R.L. Zhao \& B. Cao, Mycologia 113 (1): 199 (2020)

*Agaricus dolichocaulis R.L. Zhao \& B. Cao, Mycologia 113 (1): 199 (2020)

*Agaricus hingganensis R.L. Zhao \& B. Cao, Mycologia 113 (1): 201 (2020)

*Agaricus jilinensis R.L. Zhao \& A.Q. Liu, Phytotaxa 452 (1): 12 (2020)

*Agaricus luteopileus R.L. Zhao \& B. Cao, Mycologia 113 (1): 204 (2020)

*Agaricus megacarpus R.L. Zhao \& B. Cao, Mycologia 113 (1): 204 (2020)

*Agaricus pyricystis R.L. Zhao \& B. Cao, Mycologia 113 (1): 207 (2020)

*Agaricus subumbonatus R.L. Zhao \& B. Cao, Mycologia 113 (1): 208 (2020)

*Agaricus zhangyensis R.L. Zhao \& A.Q. Liu, Phytotaxa 452 (1): 14 (2020)

Aggregatorygma submuriforme Aptroot, Archive For Lichenology 16: 4 (2020)

Agriosomyces longus Hanafy, Lanjekar, Dhakephalkar, T.M. Callaghan, Dagar, G.W. Griff., Elshahed \& N.H. Youssef, Mycologia 112 (6): 1230 (2020)

Ahmadea dalanensis Aman \& Khalid, Stud. Fung. 5 (1): 454 (2020)

*Akanthomyces lepidopterorum W.H. Chen, Y.F. Han \& Z.Q. Liang, Phytotaxa 459 (2): 120 (2020)

*Akanthomyces neocoleopterorum W.H. Chen, Y.F. Han, J.D. Liang \& Z.Q. Liang, Phytotaxa 432 (2): 122 (2020)

Akanthomyces noctuidarum Aini, Luangsaard, Mongkols. \& Thanakitp., MycoKeys 71: 9 (2020)

Akanthomyces pyralidarum Aini, Luangsa-ard, Mongkols. \& Thanakitp., MycoKeys 71: 11 (2020)
Akanthomyces tortricidarum Aini, Luangsaard, Mongkols. \& Thanakitp., MycoKeys 71: 13 (2020)

Aklioshbomyces papillarum Hanafy, Lanjekar, Dhakephalkar, T.M. Callaghan, Dagar, G.W Griff., Elshahed \& N.H. Youssef, Mycologia 112 (6): 1231 (2020)

Albatrellopsis flettioides J. Kahn, Sydowia 72: 267 (2020)

Alishanica miscanthi Karun., C.H. Kuo \& K. D. Hyde, Mycosphere 11 (1): 461 (2020)

Allocanariomyces tritici Mehrabi, Asgari \& Zare, Mycol. Progr. 19 (12): 1420 (2020)

Alloconiothyrium camelliae H.A. Ariyaw., I. Tsai \& Thambug., Scientific Reports 10 (no. 12762): 14 (2020)

Allocryptovalsa elaeidis Konta \& K.D. Hyde, Mycosphere 11 (1): 246 (2020)

Allocryptovalsa truncata M. Niranjan \& V.V. Sarma, Mycosphere 11 (1): 572 (2020)

Allodiatrype arengae Konta \& K.D. Hyde, Mycosphere 11 (1): 249 (2020)

Allodiatrype elaeidicola Konta \& K.D. Hyde, Mycosphere 11 (1): 251 (2020)

Allodiatrype elaeidis Konta \& K.D. Hyde, Mycosphere 11 (1): 251 (2020)

Allographa grandis Kalb, Archive For Lichenology 15: 2 (2020)

Allographa medioinspersa Feuerstein \& Aptroot, Archive For Lichenology 16: 4 (2020)

Alloleptosphaeria clematidis Phukhams. \& K.D. Hyde, Fungal Diversity 10.1007/s13225-020-00448-4: 38 (2020)

*Alloneottiosporina thailandica W.J. Li \& K.D. Hyde, Fungal Diversity 100: 327 (2020) *Allophoma alba L.W. Hou, Pfenning, L. Cai \& Crous, Stud. Mycol. 96: 327 (2020)

*Allophoma anatiae L.W. Hou \& O. Yarden, Stud. Mycol. 96: 328 (2020)

Alternaria anthropophila Iturrieta-González, Gené, Alastr.-Izq. \& Dania García, Mycoses 63 (2): 217 (2020) 
Alternaria atrobrunnea Iturrieta-González, Pujol, García \& Gené, Mycoses 63 (2): 219 (2020)

Alternaria cylindrica Jun. Nishikawa \& C. Nakash., Fungal Systematics and Evolution 5: 239 (2020)

Alternaria guarroi Iturrieta-González, García, Pujol \& Gené, Mycoses 63 (2): 219 (2020)

Alternaria mirabibensis Crous, Persoonia 45: 319 (2020)

Alternaria paragomphrenae Jun. Nishikawa \& C. Nakash., Fungal Systematics and Evolution 5: 232 (2020)

Alternaria rumicis Brahmanage, Camporesi \& K.D. Hyde, Mycosphere 11: 2519 (2020)

Alternaria triangularis Jun. Nishikawa \& C. Nakash., Fungal Systematics and Evolution 5: 274 (2020)

Alternosema bostrichidis J.J. Lipa, Y.S. Tokarev \& I.V. Issi, Parasitology Research 119: 921 (2020)

Alyxoria cyanea Aptroot, Archive For Lichenology 17: 2 (2020)

Alyxoria sierramadrensis Ertz, Huereca, Salcedo-Martínez \& Tehler, Lichenologist 52 (6): 419 (2020)

Amandinea bittangabeensis Elix \& P.M. McCarthy, Australas. Lichenol. 86: 30 (2020) Amandinea clearyi Elix \& Øvstedal, Australas. Lichenol. 86: 70 (2020)

Amandinea hypohyalina Elix \& P.M. McCarthy, Australas. Lichenol. 86: 31 (2020) Amandinea madeirensis van den Boom, Elix \& Giralt, Herzogia 33: 422 (2020)

Amandinea mountmeensis Elix \& H.Mayrhofer, Australas. Lichenol. 86: 62 (2020)

Amandinea pilbarensis Elix, Australas. Lichenol. 87: 3 (2020)

*Amanita albolimbata J.E.I. Codjia, N.S. Yorou \& Zhu L. Yang, Frontiers in Microbiology 11 (no. 599047): 5 (2020)

Amanita alseides Hanss, Bull. Soc. mycol. Fr.
133: 101 (2020)

Amanita brunneofolia J.W. Jo, H.S. Kim, Y.N. Kwag \& C.S. Kim, Phytotaxa 451 (1): 28 (2020)

Amanita domingensis Angelini \& Vizzini, Persoonia 45: 327 (2020)

Amanita electra Hanss, Bull. Soc. mycol. Fr. 133: 97 (2020)

Amanita galactica Furci \& Dentinger, Index Fungorum 445: 1 (2020)

Amanita goossensfontanae Mighell \& T.W. Henkel, Mycologia 10.1080/00275514.2020.1816386: 20 (2020) Amanita luteolamellata Mighell \& T.W. Henkel, Mycologia 10.1080/00275514.2020.1816386: 18 (2020) Amanita minima Mighell \& T.W. Henkel, Mycologia

10.1080/00275514.2020.1816386: 17 (2020)

Amanita sabulicola S. Morini, A. Gennari \& Agnello, Riv. Micol. 62 (3): 241 (2020)

Amanita xenokommosis Wartchow, Pl. Biosystems 154 (5): 700 (2020)

Amarenographium ammophilicola Dayarathne, E.B.G. Jones \& K.D. Hyde, Mycosphere 11 (1): 59 (2020)

Amauroderma aurantiaca C. Sharp \& Ryvarden, Syn. Fung. 40: 109 (2020)

Amauroderma velutina Ryvarden, Syn. Fung. 40: 101 (2020)

Amesia khuzestanica Mehrabi-Koushki, Safi \& Farokhinejad, Mycol. Progr. 19 (9): 939 (2020)

Ameson iseebi N. Itoh, A. Kuboyama, M.A. Freeman, M. Katata, T. Yamakawa \& T. Yoshinagaa, J. Invert. Path. 176 (no. 107472): 1 (2020)

Amnocutis rivularis K.H. Larss. \& F.G. Oldervik, Syn. Fung. 41: 10 (2020)

Amoeboradix gromovii Karpov, López-García, Mamkaeva \& Moreira, Index Fungorum 465: 1 (2020)

Amorocoelophoma camelliae H.A. Ariyaw., I. 
Tsai \& Thambug., Scientific Reports 10 (no. 12762): 15 (2020)

*Amphichorda cavernicola Z.F. Zhang \& L. Cai, Fungal Diversity 10.1007/s13225-02000453-7: 67 (2020)

*Amphisphaeria camelliae Samarak., Jian K. Liu \& K.D. Hyde, Journal of Fungi 6 (3): 10 (2020)

Amphisphaeria curvaticonidia Samarak., Promp. \& K.D. Hyde, Journal of Fungi 6 (3): 13 (2020)

*Amphisphaeria micheliae Samarak., Jian K. Liu \& K.D. Hyde, Journal of Fungi 6 (3): 16 (2020)

*Amphisphaeria yunnanensis Dissan., J.C. Kang \& K.D. Hyde, Phytotaxa 446 (3): 147 (2020)

Amphosoma atroolivaceum Baral \& G. Marson, Monogr. Orbiliomycetes: 278 (2020)

Amphosoma macrosporum Baral \& G. Marson, Monogr. Orbiliomycetes: 281 (2020)

Amphosoma natalense Baral, Monogr. Orbiliomycetes: 277 (2020)

Amphosoma resinicola Baral \& G. Marson, Monogr. Orbiliomycetes: 274 (2020)

*Amyloceraceomyces angustisporus S.H. He, Fungal Diversity 10.1007/s13225-02000461-7: 119 (2020)

*Amylocorticium ellipsosporum S.H. He, Fungal Diversity 10.1007/s13225-02000461-7: 119 (2020)

*Anasporidesmiella manifesta Heredia, J. Delgado, K. Zhang, R.F. Castañeda \& Jian Ma, Mycotaxon 135 (4): 724 (2020)

Angustimassarina camporesii D. Pem \& K.D. Hyde, Fungal Diversity 100: 24 (2020)

*Anisomeridium guangdongense S.H. Jiang \& C. Zhang, Phytotaxa 458 (2): 168 (2020)

*Anisomeridium guangxiense S.H. Jiang \& C. Zhang, Phytotaxa 458 (2): 170 (2020)

*Annulatascus thailandensis W. Dong, H.
Zhang \& K.D. Hyde, Mycosphere 11 (1): 418 (2020)

Annulohypoxylon spougei Suwannasai, M.P. Martín, Phosri \& Whalley, Persoonia 44: 353 (2020)

Anthosulcatispora subglobosa Phukhams. \& K.D. Hyde, Fungal Diversity 10.1007/s13225-020-00448-4: 119 (2020)

*Anthracina ramosa L. Su, W. Sun \& M.C. Xiang, Journal of Fungi 6 (4, no. 187): 13 (2020)

*Anthracina saxicola L. Su, W. Sun \& M.C. Xiang, Journal of Fungi 6 (4, no. 187): 14 (2020)

*Antrodia yunnanensis M.L. Han \& Q. An, Phytotaxa 460 (1): 6 (2020)

Antrodiella nigropora Ryvarden, Syn. Fung. 40: 102 (2020)

Antrodiella subnigra Oba, Mossebo \& Ryvarden, Syn. Fung. 40: 97 (2020)

Aphelidium collabens K. Seto, Protist 171 (3, no. 125728): 5 (2020)

*Apiognomonia pseudohystrix W.J. Li, Camporesi \& K.D. Hyde, Fungal Diversity 100: 337 (2020)

Apiotrichum akiyoshidainum M. Takash., Kurakado, O. Cho, K. Kikuchi, Sugiy. \& Sugita, Int. J. Syst. Evol. Microbiol. 10.1099/ijsem.0.004277: 7 (2020)

Apiotrichum chiropterorum M. Takash., Kurakado, O. Cho, K. Kikuchi, Sugiy. \& Sugita, Int. J. Syst. Evol. Microbiol. 10.1099/ijsem.0.004277: 9 (2020)

Apiotrichum coprophilum M. Takash., Kurakado, O. Cho, K. Kikuchi, Sugiy. \& Sugita, Int. J. Syst. Evol. Microbiol. 10.1099/ijsem.0.004277: 9 (2020)

Apiotrichum otae M. Takash., Kurakado, O. Cho, K. Kikuchi, Sugiy. \& Sugita, Int. J. Syst. Evol. Microbiol. 10.1099/ijsem.0.004277: 10 (2020)

Apiotrichum xylopini S.O. Suh, C.F. Lee, Gujjari \& J.J. Zhou ex Kachalkin, Yurkov \& 
Boekhout, Stud. Mycol. 96: 132 (2020)

Aplosporella chromolaenae Mapook \& K.D. Hyde, Fungal Diversity 101: 108 (2020)

Aquadictyospora clematidis Phukhams., D.J. Bhat \& K.D. Hyde, Fungal Diversity 10.1007/s13225-020-00448-4: 17 (2020)

*Aquatospora cylindrica W. Dong, H. Zhang \& K.D. Hyde, Fungal Diversity 10.1007/s13225-020-00463-5: 61 (2020)

*Aquihelicascus songkhlaensis W. Dong, H. Zhang \& K.D. Hyde, Fungal Diversity 10.1007/s13225-020-00463-5: 144 (2020)

*Aquihelicascus yunnanensis W. Dong, H. Zhang \& K.D. Hyde, Fungal Diversity 10.1007/s13225-020-00463-5: 146 (2020)

*Aquimassariosphaeria kunmingensis W. Dong, Doilom \& K.D. Hyde, Fungal Diversity $\quad 10.1007 / \mathrm{s} 13225-020-00463-5$ : 106 (2020)

Arachnopeziza estonica T. Kosonen, Huhtinen \& K. Hansen, Persoonia 46: 39 (2020)

Arachnopeziza ptilidiophila T. Kosonen, Huhtinen \& K. Hansen, Persoonia 46: 43 (2020)

Arboricolonus simplex S. Bien \& Damm, MycoKeys 63: 130 (2020)

Archaeosporites rhyniensis C. Walker, C.J. Harper \& M. Krings, Ann. Bot. 126: 921 (2020)

Arcopilus amazonicus T.F. Sousa \& G.F. Silva, Phytotaxa 456 (2): 150 (2020)

Arrhenia fenicola C.R.J. Hay \& Thorn, Sydowia 73: 146 (2020)

Arrhenia juncorum P.-A. Moreau \& Corriol, Sydowia 73: 150 (2020)

Arrhenia leucotricha P.-A. Moreau \& Corriol, Sydowia 73: 152 (2020)

Arrhenia svalbardensis Gulden, I. Saar \& Lücking, Sydowia 73: 155 (2020)

*Arthrinium bambusicola X. Tang, K.D. Hyde \& J.C. Kang, Biodiversity Data Journal 8 (e58755): 11 (2020)

*Arthrinium chinense C.M. Tian \& N. Jiang,
Sydowia 72: 78 (2020)

Arthrinium chromolaenae Mapook \& K.D. Hyde, Fungal Diversity 101: 138 (2020)

*Arthrinium neogarethjonesii D.Q. Dai \& K.D. Hyde, Mycosphere 11 (1): 424 (2020)

Arthrinium sorghi J.D.P. Bezerra, C.M Gonçalves \& C.M. Souza-Motta, Fungal Diversity 10.1007/s13225-020-00461-7: 73 (2020)

Arthroderma melbournense Hainsworth \& Hubka, Medical Mycol. myaa057: 7 (2020)

Arthrographis grakistii Giraldo López \& Hern.-Restr., Fungal Systematics and Evolution 6: 13 (2020)

*Ascagilis submersa W. Dong, H. Zhang \& K.D. Hyde, Fungal Diversity 10.1007/s13225-020-00463-5: 29 (2020)

*Ascagilis thailandensis W. Dong, H. Zhang \& K.D. Hyde, Fungal Diversity 10.1007/s13225-020-00463-5: 31 (2020)

*Ascochyta benningiorum Hern.-Restr., L.W. Hou, L. Cai \& Crous, MycoKeys 65: 71 (2020)

Ascochyta kamchatica M.M. Gomzhina \& E.L. Gasich, Nova Hedwigia 111 (1-2): 143 (2020)

*Ascochyta neopisi W.J. Li, Camporesi \& K.D. Hyde, Fungal Diversity 100: 351 (2020)

*Ascochyta pilosella L.W. Hou, L. Cai \& Crous, Stud. Mycol. 96: 381 (2020)

Ascotaiwania latericolla Réblová, Hern.-Restr. \& J. Fourn., Stud. Mycol. 95: 444 (2020)

Aspergillus agricola Pummi Singh, K.A. Callicott, M.J. Orbach \& P.J. Cotty, Frontiers in Microbiology 10.3389/fmicb.2020.01236: 7 (2020)

Aspergillus amethystinus F. Sklenar, S.W. Peterson \& Hubka, Mycologia 112 (2): 355 (2020)

Aspergillus banksianus Pitt, Persoonia 44: 355 (2020)

Aspergillus barbosae A.C.R. Barros-Correia, R.N. Barbosa, Houbraken \& Souza-Motta, 
Mycol. Progr. 19 (9): 892 (2020)

Aspergillus burnettii Pitt, Fungal Genetics Biol. 143 (no. 103435): 5 (2020)

Aspergillus coloradensis F. Sklenar, Jurjević \& Hubka, Mycologia 112 (2): 357 (2020)

Aspergillus croceiaffinis F. Sklenar, Jurjević \& Hubka, Mycologia 112 (2): 359 (2020)

Aspergillus curvatus Al-Bedak \& Moubasher, Asian Journal of Mycology 3 (1): 327 (2020)

Aspergillus dipodomyus F. Sklenar, Jurjević \& Hubka, Mycologia 112 (2): 360 (2020)

Aspergillus elsenburgensis Visagie, S.M. Romero \& Houbraken, Stud. Mycol. 95: 275 (2020)

Aspergillus gaarensis Al-Bedak \& Moubasher, Index Fungorum 451: 1 (2020)

Aspergillus heldtiae Visagie, Stud. Mycol. 95: 276 (2020)

Aspergillus hydei Doilom, Frontiers in Microbiology 11 (no. 585215): 4 (2020)

Aspergillus krugeri Visagie, Stud. Mycol. 95: 279 (2020)

Aspergillus kumbius Pitt, Persoonia 44: 359 (2020)

*Aspergillus limoniformis Z.F. Zhang \& L. Cai, Fungal Diversity 10.1007/s13225-02000453-7: 49 (2020)

Aspergillus longistipitatus F. Sklenar, Jurjević \& Hubka, Mycologia 112 (2): 363 (2020)

Aspergillus luteorubrus Pitt, Persoonia 44: 361 (2020)

Aspergillus magaliesburgensis Visagie, Stud. Mycol. 95: 285 (2020)

Aspergillus malvicolor A.D. Hocking, Persoonia 44: 363 (2020)

Aspergillus nanangensis Pitt, Persoonia 44: 365 (2020)

Aspergillus oxumiae C.N. Figueiredo, L.S. Sales, Y.F. Figueiredo, J.P. Andrade \& J.T. De Souza, Persoonia 44: 357 (2020)

*Aspergillus phialiformis Z.F. Zhang \& L. Cai, Fungal Diversity 10.1007/s13225-02000453-7: 51 (2020)
*Aspergillus phialosimplex Z.F. Zhang \& L. Cai, Fungal Diversity 10.1007/s13225-02000453-7: 51 (2020)

Aspergillus purpureocrustaceus Visagie, Stud. Mycol. 95: 285 (2020)

Aspergillus recifensis A.C.R. Barros-Correia, R.N. Barbosa, Houbraken \& Souza-Motta, Mycol. Progr. 19 (9): 895 (2020)

Aspergillus seifertii Visagie \& N. Yilmaz, Stud. Mycol. 95: 289 (2020)

Aspergillus sigurros Visagie, Stud. Mycol. 95: 289 (2020)

Aspergillus stelliformis F. Sklenar, Jurjević \& Hubka, Mycologia 112 (2): 363 (2020)

Aspergillus toxicus Pummi Singh, K.A. Callicott, M.J. Orbach \& P.J. Cotty, Frontiers in Microbiology 10.3389/fmicb.2020.01236: 10 (2020)

Aspergillus vinaceus Ferranti, Iamanaka, Frisvad, O. Puel \& J.J. da Silva, Journal of Fungi 6 (no. 371): 14 (2020)

Aspicilia bauvetiae $\mathrm{Cl}$. Roux, M. Bertrand \& Poumarat, Catalogue des lichens et champignons lichénicoles de France métropolitaine. 3e édition revue et augmentée 1: 1261 (2020)

Aspicilia clercii Cl. Roux \& M. Bertrand, Catalogue des lichens et champignons lichénicoles de France métropolitaine. 3e édition revue et augmentée 1: 1268 (2020)

Aspicilia navarroi $\mathrm{Cl}$. Roux \& $\mathrm{M}$. Bertrand, Catalogue des lichens et champignons lichénicoles de France métropolitaine. 3e édition revue et augmentée 1: 1273 (2020)

Aspicilia stalagmitica Paukov \& Davydov, Turczaninowia 23 (1): 9 (2020)

Asproinocybe daleyae J.A. Cooper, Muelleria 38: 78 (2020)

Asterodiscus mangrovei Dayarathne, E.B.G. Jones \& K.D. Hyde, Mycosphere 11 (1): 86 (2020)

Astrothelium ochraceum Aptroot, Archive For Lichenology 17: 3 (2020) 
Atrocalyx quercus Tibpromma \& K.D. Hyde, Fungal Diversity $10.1007 / \mathrm{s} 13225-020-$ 00458-2: 7 (2020)

Atrophysma cyanomelanos T. Sprib., Lichenologist 52 (2): 85 (2020)

Aureobasidium mustum C. Onetto, S. Schmidt, M. Roach \& A. Borneman, FEMS Yeast Res. 20 (6): foaa052, 4 (2020)

Aureobasidium uvarum C. Onetto, S. Schmidt, M. Roach \& A. Borneman, FEMS Yeast Res. 20 (6): foaa052, 4 (2020)

Aureobasidium vineae C. Onetto, S. Schmidt, M. Roach \& A. Borneman, FEMS Yeast Res. 20 (6): foaa052, 4 (2020)

Aureoboletus garciae Ayala-Vásquez \& Aguirre-Acosta, Sydowia 72: 270 (2020)

Aureoconidiella foliicola Hern.-Restr. \& Crous, Fungal Systematics and Evolution 6: 11 (2020)

*Aurifilum terminaliae Wen Wang \& S.F. Chen, Persoonia 45: 124 (2020)

Austroboletus asper K. Syme, Bonito, T. Lebel, Fechner \& Halling, Persoonia 45: 329 (2020)

*Auxarthron chinense Z.F. Zhang \& L. Cai, Fungal Diversity 10.1007/s13225-02000453-7: 55 (2020)

*Auxarthron guangxiense Z.F. Zhang \& L. Cai, Fungal Diversity 10.1007/s13225-02000453-7: 57 (2020)

*Auxarthronopsis globiasca Z.F. Zhang \& L. Cai, Fungal Diversity 10.1007/s13225-02000453-7: 58 (2020)

*Auxarthronopsis pedicellaris Z.F. Zhang \& L. Cai, Fungal Diversity 10.1007/s13225-02000453-7: 60 (2020)

*Auxarthronopsis pulverea Z.F. Zhang \& L. Cai, Fungal Diversity 10.1007/s13225-02000453-7: 61 (2020)

*Auxarthronopsis stercicola Z.F. Zhang \& L. Cai, Fungal Diversity 10.1007/s13225-02000453-7: 63 (2020)

Babjevia hyphasca A. Yamaz. \& H. Kawas., Int. J. Syst. Evol. Microbiol. 10.1099/ijsem.0.003924: 17 (2020)

Babjevia hyphoforaminiformans A. Yamaz. \& H. Kawas., Int. J. Syst. Evol. Microbiol. 10.1099/ijsem.0.003924: 17 (2020)

Babosia variospora D.G. Knapp, Zagyva, Trappe \& Kovács, Mycologia 112 (4): 811 (2020)

Bacidia gigantensis Lendemer, McCune \& McMullin, Bryologist 123 (2): 220 (2020)

Bacidia thiersiana Lendemer, Bryologist 123 (1): 40 (2020)

Bacidina circumpulla S. Ekman, Lichenologist 52 (2): 88 (2020)

Bacidina pallidocarpa van den Boom \& Magain, Plant and Fungal Systematics 65 (1): 170 (2020)

Bacidina violacea van den Boom \& Magain, Plant and Fungal Systematics 65 (1): 171 (2020)

Backusella australiensis Urquhart \& Douch, Persoonia 46: 14 (2020)

Backusella liffmaniae Urquhart \& Douch, Persoonia 46: 18 (2020)

Backusella luteola Urquhart \& Douch, Persoonia 46: 19 (2020)

Backusella macrospora Urquhart \& Douch, Persoonia 46: 19 (2020)

Backusella mclennaniae Urquhart \& Douch, Persoonia 46: 19 (2020)

Backusella morwellensis Urquhart \& Douch, Persoonia 46: 19 (2020)

Backusella parvicylindrica Urquhart \& Douch, Persoonia 46: 19 (2020)

Backusella psychrophila Urquhart \& Douch, Persoonia 46: 20 (2020)

Backusella tarrabulga Urquhart \& Douch, Persoonia 46: 21 (2020)

Backusella westeae Urquhart \& Douch, Persoonia 46: 22 (2020)

Bactrodesmium pulcherrimum R.F. Castañeda, F. Espinoza \& D. Sosa, Mycotaxon 134 (4): 629 (2020)

Baculifera confusa Elix, Australas. Lichenol. 
87: 4 (2020)

Baidera mauritiana Ertz \& Diederich, Plant and Fungal Systematics 65 (1): 22 (2020)

*Balsamia lishanensis L. Fan \& Y.Y. Xu, MycoKeys 67: 87 (2020)

*Bambusicola aquatica W. Dong, H. Zhang \& K.D. Hyde, Fungal Diversity 10.1007/s13225-020-00463-5: 72 (2020)

Bambusicola ficuum N.I. de Silva \& K.D. Hyde, Mycosphere 11 (1): 2487 ['7'] (2020)

*Bannozyma arctica Vishniac \& M. Takash. ex Q.M. Wang, F.Y. Bai, M. Groenew. \& Boekhout, Stud. Mycol. 96: 132 (2020)

Bapalmuia microspora Kalb, Archive For Lichenology 15: 3 (2020)

Barrmaelia serenoae Crous, Fungal Systematics and Evolution 6: 179 (2020)

Basidiodendron alni Spirin \& Malysheva, Botany 98: 627 (2020)

Basidiodendron caucasicum Spirin, Malysheva \& Kotiranta, Botany 98: 627 (2020)

Basidiodendron gelatinosum G. Gruhn \& T. Rödel, Bull. trimest. Soc. mycol. Fr. 133 (34): 237 (2020)

Basidiodendron globisporum Spirin \& Malysheva, Botany 98: 632 (2020)

Basidiodendron iniquum Alvarenga \& K.H. Larss., Botany 98: 633 (2020)

Basidiodendron microsporum Spirin, Malysheva \& Kotiranta, Botany 98: 634 (2020)

Basidiodendron olivaceum Spirin \& Malysheva, Botany 98: 635 (2020)

Basidiodendron parile Spirin \& Malysheva, Botany 98: 635 (2020)

Basidiodendron pelinum Alvarenga \& K.H. Larss., Botany 98: 636 (2020)

Basidiodendron remotum Spirin, Malysheva \& Kotiranta, Botany 98: 636 (2020)

Basidiodendron salebrosum Spirin \& Malysheva, Botany 98: 636 (2020)

*Basidioradulum mayi Xue W. Wang \& L.W.
Zhou, Scientific Reports 10 (no. 102): 2 (2020)

*Basidioradulum tasmanicum Xue W. Wang \& L.W. Zhou, Scientific Reports 10 (no. 102): 2 (2020)

Batnamyces globulariicola Noumeur, Mycol. Progr. 19 (6): 493 (2020)

Beauveria mimosiformis Khons., Thanakitp., Kobmoo \& Luangsa-ard, Mycol. Progr. 19 (4): 305 (2020)

*Begerowomyces foliicola Q.M. Wang, F.Y. Bai \& A.H. Li, Stud. Mycol. 96: 117 (2020)

Bellamyces quercus Crous, Coppins \& U. Braun, Stud. Mycol. 96: 200 (2020)

*Beltrania dushanensis C.G. Lin, Jian K. Liu \& K.D. Hyde, Asian Journal of Mycology 3 (1): 233 (2020)

*Beltraniella brevis C.G. Lin, Jian K. Liu \& K.D. Hyde, Asian Journal of Mycology 3 (1): 235 (2020)

Beltraniella podocarpi Crous, Persoonia 44: 335 (2020)

*Beltraniella ramosiphora C.G. Lin \& K.D. Hyde, Mycosphere 11 (1): 444 (2020)

Benniella erionia Liber \& Bonito, Fungal Diversity 10.1007/s13225-020-00455-5: 15 (2020)

*Bensingtonia pseudorectispora Q.M. Wang, F.Y. Bai \& A.H. Li, Stud. Mycol. 96: 107 (2020)

*Bensingtonia wuzhishanensis Q.M. Wang, F.Y. Bai \& A.H. Li, Stud. Mycol. 96: 107 (2020)

Bergerella atrofusca Diederich \& Lawrey, Bryologist 123 (2): 159 (2020)

Bhagirathimyces himalayensis S.M. Singh \& S.K. Singh, Fungal Diversity 100: 95 (2020)

Biatoropsis millanesiana Diederich \& Wedin, Plant and Fungal Systematics 65 (1): 25 (2020)

Biatriospora borsei B. Devadatha, V.V. Sarma, Mycosphere 11 (1): 1768 (2020)

Bimuria omanensis Wijesinghe, Wanas., Al- 
Sadi, K.D. Hyde \& Maharachch., Phytotaxa 449 (2): 104 (2020)

Biscogniauxia mangiferae Samarak. \& K.D. Hyde, Mycosphere 11 (1): 611 (2020)

Bjerkandera albocinerea Motato-Vásq., Robledo \& Gugliotta, Plant Ecology and Evolution 153 (2): 234 (2020)

Blackwellomyces aurantiacus Mongkols., Noisrip., Himaman \& Luangsa-ard, Mycol. Progr. 19 (9): 962 (2020)

Blackwellomyces calendulinus Mongkols., Noisrip., Khons. \& Luangsa-ard, Mycol. Progr. 19 (9): 967 (2020)

Blackwellomyces minutus Mongkols., Noisrip., Himaman \& Luangsa-ard, Mycol. Progr. 19 (9): 968 (2020)

Blackwellomyces roseostromatus Mongkols., Noisrip., Khonsanit \& Luangsa-ard, Mycol. Progr. 19 (9): 968 (2020)

Blastenia afroalpina Vondrák, Journal of Systematics and Evolution 58 (3): 313 (2020)

Blastenia anatolica Halıc1, Arup \& Vondrák, Journal of Systematics and Evolution 58 (3): 314 (2020)

Blastenia caucasica I.V. Frolov \& Vondrák, Journal of Systematics and Evolution 58 (3): 315 (2020)

Blastenia gennargentuae Vondrák, Journal of Systematics and Evolution 58 (3): 318 (2020)

Blastenia herbidella subsp. acidophila Urbanavichene \& Vondrák, Journal of Systematics and Evolution 58 (3): 319 (2020)

Blastenia lauri Vondrák, Journal of Systematics and Evolution 58 (3): 319 (2020)

Blastenia monticola Arup \& Vondrák, Journal of Systematics and Evolution 58 (3): 320 (2020)

Blastenia palmae Vondrák, Journal of Systematics and Evolution 58 (3): 320 (2020)

Blastenia psychrophila Halıc1 \& Vondrák, Journal of Systematics and Evolution 58 (3): 320 (2020)

Blastenia purpurea Vondrák, Journal of
Systematics and Evolution 58 (3): 321 (2020)

Blastenia relicta Arup \& Vondrák, Journal of Systematics and Evolution 58 (3): 321 (2020)

Blastenia remota Obermayer \& Vondrák, Journal of Systematics and Evolution 58 (3): 321 (2020)

Blastenia xerothermica Vondrák, Arup \& I.V. Frolov, Journal of Systematics and Evolution 58 (3): 322 (2020)

Blastenia xerothermica subsp. macaronesica Vondrák, Journal of Systematics and Evolution 58 (3): 323 (2020)

*Blastobotrys baotianmanensis F.L. Hui \& Huang, Int. J. Syst. Evol. Microbiol. 70 (7): 4222 (2020)

*Blastobotrys xishuangbannaensis F.L. Hui \& Huang, Int. J. Syst. Evol. Microbiol. 70 (7): 4222 (2020)

Blastomyces emzantsi Maphanga, Govender, Birkhead \& Frean, J. Clin. Microbiol. 58 (3): e01661-19, 14 (2020)

Blumenavia baturitensis Melanda, M.P. Martín \& Baseia, PLoS ONE 15 (5): e0232467, 12 (2020)

Blumenavia crucis-hellenicae G. Coelho, Sulzbacher, Grebenc \& Cortez, PLoS ONE 15 (5): e0232467, 18 (2020)

Blumenavia heroica Melanda, Baseia \& M.P. Martín, PLoS ONE 15 (5): e0232467, 19 (2020)

*Boekhoutia sterigmata Q.M. Wang, F.Y. Bai \& A.H. Li, Stud. Mycol. 96: 110 (2020)

Boeremia maritima Dayarathne, E.B.G. Jones \& K.D. Hyde, Mycosphere 11 (1): 36 (2020)

Bogoriella complexoluminata Aptroot \& Lücking, Fungal Diversity 10.1007/s13225020-00462-6: 165 (2020)

Bolbea parasitica Buaya \& Thines, Fungal Systematics and Evolution 6: 132 (2020)

*Botryosphaeria guttulata Y.Y. Chen, A.J. Dissanayake \& J.K. Liu, Phytotaxa 454 (3): 191 (2020)

*Botryosphaeria puerensis G.Q. Li \& S.F. 
Chen, IMA Fungus 11 (no. 22): 33 (2020)

Botryotrichum iranicum A. Alidadi, Mycol. Progr. 19 (12): 1578 (2020)

*Bradymyces pullus L. Su, W. Sun \& M.C. Xiang, Journal of Fungi 6 (4, no. 187): 15 (2020)

*Bradymyces yunnanensis L. Su, W. Sun \& M.C. Xiang, Journal of Fungi 6 (4, no. 187): $16(2020)$

Brijax amictus M. Krings \& C.J. Harper, Review of Palaeobotany and Palynology 281 (no. 104287): 5 (2020)

Brocchiosphaera bulbiformis K. Yamag., Chuaseehar. \& Nakagiri, Mycoscience 61 (6): 280 (2020)

Brunneoclavispora camporesii Boonmee \& Phookamsak, Fungal Diversity 100: 61 (2020)

Brunneofusispora clematidis Phukhams. \& K.D. Hyde, Fungal Diversity 10.1007/s13225-020-00448-4: 63 (2020)

Bryoclavula phycophila $\mathrm{H}$. Masumoto \& Y. Degawa, Mycol. Progr. 19 (7): 708 (2020)

Bryorbilia arenicola E. Rubio \& Baral, Monogr. Orbiliomycetes: 302 (2020)

Bryostigma huriellum S.Y. Kondr. \& Hur, Acta bot. hung. 62 (3-4): 227 (2020)

Buellia acervicola Elix \& Kantvilas, Australas. Lichenol. 87: 20 (2020)

Buellia arida Elix, Australas. Lichenol. 87: 5 (2020)

Buellia bularmialensis Elix \& H.Mayrhofer, Australas. Lichenol. 86: 63 (2020)

Buellia cravenii Elix, Australas. Lichenol. 87: 6 (2020)

Buellia dayboroana Elix \& H.Mayrhofer, Australas. Lichenol. 86: 62 (2020)

Buellia eldridgei Elix, Australas. Lichenol. 87: 7 (2020)

*Buellia endoflavida Xin Y. Wang \& Li J. Li, Bryologist 123 (3): 435 (2020)

Buellia gibstoneorum Brodo \& Sheard, Opuscula Philolichenum 19: 164 (2020)
Buellia kowenensis Elix \& P.M. McCarthy, Australas. Lichenol. 87: 8 (2020)

Buellia lordhowensis Elix, Australas. Lichenol. 87: 8 (2020)

*Buellia microareolata Xin Y. Wang \& Li S. Wang, Bryologist 123 (3): 437 (2020)

Buellia neohalonia Elix \& H. Mayrhofer, Australas. Lichenol. 86: 64 (2020)

Buellia paradisana Elix \& Kantvilas, Australas. Lichenol. 87: 21 (2020)

Buellia phillipensis Elix, Australas. Lichenol. 87: 9 (2020)

Buellia quarryana Elix \& P.M. McCarthy, Australas. Lichenol. 86: 32 (2020)

*Bulleribasidium cremeum Q.M. Wang, F.Y. Bai \& A.H. Li, Stud. Mycol. 96: 91 (2020)

*Bulleribasidium elongatum Q.M. Wang, F.Y. Bai \& A.H. Li, Stud. Mycol. 96: 93 (2020)

*Bulleribasidium panici Fungsin, M. Takash. \& Nakase ex Xin Zhan Liu, F.Y. Bai, M. Groenew. \& Boekhout, Stud. Mycol. 96: 133 (2020)

*Bulleribasidium phyllophilum Q.M. Wang, F.Y. Bai \& A.H. Li, Stud. Mycol. 96: 91 (2020)

*Bulleribasidium phyllostachydis Q.M. Wang, F.Y. Bai \& A.H. Li, Stud. Mycol. 96: 90 (2020)

*Bulleribasidium pseudopanici Q.M. Wang, F.Y. Bai \& A.H. Li, Stud. Mycol. 96: 91 (2020)

*Bulleribasidium siamense Fungsin, M. Takash. \& Nakase ex Q.M. Wang, F.Y. Bai, Boekhout \& Nakase, Stud. Mycol. 96: 133 (2020)

Burrowsia cataractae Fryday \& I. Medeiros, S. Afr. J. Bot. 132: 473 (2020)

*Byssoloma brunneidiscum W.C. Wang \& J.C. Wei, Lichenologist 52 (5): 391 (2020)

*Byssoloma erythrodiscocarpum W.C. Wang \& J.C. Wei, Lichenologist 52 (5): 393 (2020)

*Byssoloma melanodiscocarpum W.C. Wang \& J.C. Wei, Lichenologist 52 (5): 394 (2020) 
Cadophora africana Damm \& S. Bien, MycoKeys 63: 132 (2020)

Cadophora constrictospora Koukol \& MaciáVicente, IMA Fungus 11 (no. 16): 9 (2020)

Cadophora echinata Koukol \& Maciá-Vicente, IMA Fungus 11 (no. 16): 12 (2020)

Cadophora fallopiae Crous \& Akulov, Fungal Systematics and Evolution 6: 180 (2020)

Cadophora fascicularis Koukol \& MaciáVicente, IMA Fungus 11 (no. 16): 12 (2020) Cadophora ferruginea Koukol \& MaciáVicente, IMA Fungus 11 (no. 16): 13 (2020)

Cadophora gamsii Koukol \& Maciá-Vicente, IMA Fungus 11 (no. 16): 14 (2020)

Cadophora obovata Koukol \& Maciá-Vicente, IMA Fungus 11 (no. 16): 15 (2020)

Cadophora prunicola Damm \& S. Bien, MycoKeys 63: 140 (2020)

Cadophora ramosa Damm \& S. Bien, MycoKeys 63: 142 (2020)

Cadophora variabilis Koukol \& MaciáVicente, IMA Fungus 11 (no. 16): 17 (2020)

*Calenia flavescens Z.F. Jia, J. Fungal Res. 18 (3): 150 (2020)

*Calenia verrucosa Z.F. Jia, J. Fungal Res. 18 (3): 151 (2020)

Caliciopsis sambaibae J.L. Bezerra, M.P. Melo \& Beserra, Mycotaxon 135 (1): 99 (2020)

Callistosporium pseudofelleum Vizzini, Matheny, Consiglio \& M. Marchetti, Fungal Diversity 101: 236 (2020)

Calocybella dicholamellata K.P.D. Latha, K.N.A. Raj \& Manimohan, Cryptog. Mycol. 41 (8): 150 (2020)

Calophoma humulicola Chaiwan, T.C. Bulgakov, Jayaward \& K.D. Hyde, Asian Journal of Mycology 3 (1): 74 (2020)

*Calophoma parvula L.W. Hou, L. Cai \& Crous, Stud. Mycol. 96: 376 (2020)

Caloplaca edwardiana J.R. Hoffm. \& Lendemer, Bryologist 123 (2): 226 (2020)

Caloplaca nigra Bungartz \& Søchting, Plant and Fungal Systematics 65 (2): 529 (2020)
Caloplaca nothocitrina S.Y. Kondr. \& Hur, Acta bot. hung. 62 (3-4): 228 (2020)

Caloplaca nothoholocarpa S.Y. Kondr. \& Hur, Acta bot. hung. 62 (3-4): 231 (2020)

Caloplaca orloviana S.Y. Kondr., Acta bot. hung. 62 (1-2): 71 (2020)

Caloplaca patagoniensis S.Y. Kondr., S.O. Oh \& Hur, Acta bot. hung. 62 (3-4): 234 (2020)

Caloplaca rajasthanica S.Y. Kondr., Upreti \& G.P. Sinha, Acta bot. hung. 62 (3-4): 340 (2020)

Caloplaca ulleungensis S.Y. Kondr., Lökös \& Hur, Acta bot. hung. 62 (3-4): 236 (2020)

Calvatia baixaverdensis R.L. Oliveira, R.J. Ferreira, P. Marinho, M.P. Martín \& Baseia, Persoonia 44: 367 (2020)

Camarosporidiella camporesii Tibpromma \& K.D. Hyde, Fungal Diversity 100: 26 (2020)

Cambaraspora floridanus Bojkoa, Behringer, Molere, Strattond \& Reisingerd, J. Invert. Path. 171 (no. 107345): 8 (2020)

*Camporesiomyces mali D.P. Wei \& K.D. Hyde, Fungal Diversity 100: 139 (2020)

*Camposporium appendiculatum D.F. Bao, Z.L. Luo, K.D. Hyde \& H.Y. Su, Fungal Diversity 100: 77 (2020)

*Camposporium multiseptatum D.F. Bao, Z.L. Luo, K.D. Hyde \& H.Y. Su, Fungal Diversity 100: 82 (2020)

*Camposporium septatum N.G. Liu, J.K. Liu \& K.D. Hyde, Fungal Diversity 100: 85 (2020)

Camptobasidium gelus V. de Garcia, Trochine, Uetake \& Libkind, Int. J. Syst. Evol. Microbiol. 70 (6): 3716 (2020)

*Canalisporium aquaticium J. Yang \& K.D. Hyde, Fungal Diversity 100: 209 (2020)

Candida pellucidi Glushakova, Tomashevskaya \& Kachalkin, Persoonia 44: 369 (2020)

Candolleomyces eurysporus A. Karich, E. Büttner \& R. Ullrich, Öst. Z. Pilzk. 28: 85 (2020) 
Canomyces reticulatus Rahul Sharma \& Shouche, Mycopathologia 185 (1): 78 (2020)

Cantharellus albidosquamosus Buyck, T.W. Henkel \& V. Hofst., Cryptog. Mycol. 41 (4): $74(2020)$

*Cantharellus albus S.P. Jian \& B. Feng, Phytotaxa 470 (2): 137 (2020)

Cantharellus betularum Voitk \& Thorn, Persoonia 45: 331 (2020)

Cantharellus citrinus Buyck, R. Ryoo \& Antonín, MycoKeys 76: 35 (2020)

Cantharellus curvatus Buyck, R. Ryoo \& Antonín, MycoKeys 76: 39 (2020)

Cantharellus violaceoflavescens De Kesel, Guelly, L.A. Parra \& Buyck, Cryptog. Mycol. 41 (10): 172 (2020)

Cantharellus xanthocyaneus Ndolo Ebika \& Buyck, MycoKeys 41 (10): 169 (2020)

Capellomyces elongatus Hanafy, Lanjekar, Dhakephalkar, T.M. Callaghan, Dagar, G.W. Griff., Elshahed \& N.H. Youssef, Mycologia 112 (6): 1232 (2020)

Capellomyces foraminis Hanafy, Lanjekar, Dhakephalkar, T.M. Callaghan, Dagar, G.W. Griff., Elshahed \& N.H. Youssef, Mycologia 112 (6): 1232 (2020)

Capnodium alfenasii Abdollahz. \& Crous, Stud. Mycol. 95: 394 (2020)

Capnodium blackwelliae Abdollahz. \& Crous, Stud. Mycol. 95: 395 (2020)

Capnodium gamsii Abdollahz. \& Crous, Stud. Mycol. 95: 395 (2020)

Capnodium neocoffeicola Abdollahz. \& Crous, Stud. Mycol. 95: 396 (2020)

Capnodium paracoffeicola Abdollahz. \& Crous, Stud. Mycol. 95: 396 (2020)

Carcinomyces arundinariae Fungsin, M. Takash. \& Nakase ex Yurkov, Stud. Mycol. 96: 133 (2020)

*Carlosrosaea foliicola Q.M. Wang, F.Y. Bai \& A.H. Li, Stud. Mycol. 96: 85 (2020)

*Carlosrosaea simaoensis Q.M. Wang, F.Y. Bai \& A.H. Li, Stud. Mycol. 96: 85 (2020)
Carneothele sphagnicola Fryday, M. Svenss. \& Holien, Lichenologist 52 (2): 91 (2020)

*Caryospora submersa W. Dong, H. Zhang \& K.D. Hyde, Fungal Diversity 10.1007/s13225-020-00463-5: 66 (2020)

*Catathelasma laorentou Z.W. Ge, MycoKeys 62: 129 (2020)

*Catathelasma subalpinum Z.W. Ge, MycoKeys 62: 132 (2020)

*Catenuliconidia uniseptata N.G. Liu \& K.D. Hyde, Fungal Diversity 10.1007/s13225020-00461-7: 97 (2020)

Catinaria occidentalis Van den Boom, Ascomycete.org 12 (5): 200 (2020)

Celerioriella umnquma C.F.J. Spies, van Jaarsveld, L. Mostert \& Halleen, Persoonia 45: 203 (2020)

Celoporthe hauoliensis Kamgan, Jol. Roux \& Marinc., MycoKeys 76: 64 (2020)

Celoporthe hawaiiensis Kamgan, Jol. Roux \& Marinc., MycoKeys 76: 66 (2020)

*Celoporthe paradisiaca S.F. Chen \& Marinc., MycoKeys 76: 68 (2020)

Cenangiopsis desae B. Perić, Ascomycete.org 12 (6): 227 (2020)

Cenangiopsis rosae B. Perić, Ascomycete.org 12 (6): 239 (2020)

Ceratocystiopsis synnemata B. Strzałka, R. Jankowiak \& G. Hausner, MycoKeys 68: 38 (2020)

Ceratocystis quercicola D.H. Lee, S.E. Cho, Marinc. \& M.J. Wingf., Mycobiology 48 (4): 248 (2020)

*Ceriporia arbuscula C.C. Chen \& Sheng H. Wu, Mycologia 112 (1): 66 (2020)

Ceriporia globospora Ryvarden, Syn. Fung. 42: 28 (2020)

Ceriporiopsis gabonensis Decock \& Ryvarden, Syn. Fung. 42: 7 (2020)

Ceriporiopsis nigroeffusus Oba, Mossebo \& Ryvarden, Syn. Fung. 40: 100 (2020)

Chaenothecopsis jordaniana Gockman \& Selva, Bryologist 123 (2): 246 (2020) 
Chaenothecopsis penningtonensis Gockman \& Selva, Bryologist 123 (2): 248 (2020)

*Chaetasbolisia argentina L.W. Hou, L. Cai \& Crous, Stud. Mycol. 96: 332 (2020)

Chaetocapnodium indonesiacum Abdollahz. \& Crous, Stud. Mycol. 95: 398 (2020)

Chaetocapnodium insulare Abdollahz. \& Crous, Stud. Mycol. 95: 399 (2020)

Chaetocapnodium summerellii Abdollahz. \& Crous, Stud. Mycol. 95: 400 (2020)

Chaetocapnodium tanzanicum Abdollahz. \& Crous, Stud. Mycol. 95: 401 (2020)

Chaetocapnodium thailandense Abdollahz. \& Crous, Stud. Mycol. 95: 401 (2020)

*Chaetophiophoma sorbi W.J. Li, Camporesi \& K.D. Hyde, Fungal Diversity 100: 382 (2020)

Chaetopsina aurantisalinicola Dayarathne, E.B.G. Jones \& K.D. Hyde, Mycosphere 11 (1): 102 (2020)

Chaetopsina gautengina Crous, Fungal Systematics and Evolution 6: 181 (2020)

Chaetopsina pnagiana Lechat \& J. Fourn., Ascomycete.org 12 (1): 1 (2020)

Chaetosphaeronema clematidicola Phukhams., Ertz, Gerstmans \& K.D. Hyde, Fungal Diversity 10.1007/s13225-02000448-4: 75 (2020)

Chaetosphaeronema clematidis Phukhams., Ertz, Gerstmans \& K.D. Hyde, Fungal Diversity 10.1007/s13225-020-00448-4: 75 (2020)

Chaetothyrina spondiadis Fuentes-Aponte, K. Kim \& Romberg, Persoonia 45: 333 (2020)

Chalciporus pseudopiperatus Klofac \& Krisai-Greilhuber, Öst. Z. Pilzk. 28: 76 (2020)

Chapsa alletii Diederich \& Ertz, Plant and Fungal Systematics 65 (1): 28 (2020)

Chapsa canaimae Kalb, Archive For Lichenology 18: 2 (2020)

Chiodecton xanthonosorediatum Aptroot, Archive For Lichenology 17: 4 (2020)
Chloridium pini Crous \& Akulov, Fungal Systematics and Evolution 6: 182 (2020)

Chloridium salinicola Dayarathne \& E.B.G. Jones, Mycosphere 11 (1): 122 (2020)

*Chloridium submersum Z.L. Luo, K.D. Hyde \& H.Y. Su, Mycosphere 11 (1): 502 (2020)

Chlorophyllum levantinum Loizides, $\mathrm{P}$. Alvarado \& Konstantinou, Mycologia 112 (2): 413 (2020)

Chlorophyllum squamulosum A.K. Dutta, Soumili Bera \& K. Acharya, Phytotaxa 451 (2): 121 (2020)

Chromolaenicola chiangraiensis Mapook \& K.D. Hyde, Fungal Diversity 101: 21 (2020) Chromolaenicola clematidis Phukhams. \& K.D. Hyde, Fungal Diversity 10.1007/s13225-020-00448-4: 31 (2020)

Chromolaenicola lampangensis Mapook \& K.D. Hyde, Fungal Diversity 101: 23 (2020) Chromolaenicola nanensis Mapook \& K.D. Hyde, Fungal Diversity 101: 25 (2020)

Chromolaenicola thailandensis Mapook \& K.D. Hyde, Fungal Diversity 101: 28 (2020) Chromolaenomyces appendiculatus Mapook \& K.D. Hyde, Fungal Diversity 101: 97 (2020)

Chroogomphus pakistanicus M. Kiran \& A.N. Khalid, MycoKeys 66: 28 (2020)

Chroogomphus pruinosus M. Kiran \& A.N. Khalid, MycoKeys 66: 31 (2020)

*Chrysosporium pallidum Z.F. Zhang \& L. Cai, Fungal Diversity 10.1007/s13225-02000453-7: 64 (2020)

Chrysothrix bergeri LaGreca, Plant and Fungal Systematics 65 (2): 510 (2020)

*Chrysozyma cylindrica Q.M. Wang, F.Y. Bai \& A.H. Li, Stud. Mycol. 96: 129 (2020)

*Chrysozyma flava Q.M. Wang, F.Y. Bai \& A.H. Li, Stud. Mycol. 96: 130 (2020)

*Chrysozyma fusiformis Q.M. Wang, F.Y. Bai \& A.H. Li, Stud. Mycol. 96: 129 (2020)

*Chrysozyma iridis Q.M. Wang, F.Y. Bai \& A.H. Li, Stud. Mycol. 96: 127 (2020) 
*Chrysozyma pseudogriseoflava Q.M. Wang, F.Y. Bai \& A.H. Li, Stud. Mycol. 96: 126 (2020)

*Chrysozyma rhododendri Q.M. Wang, F.Y. Bai \& A.H. Li, Stud. Mycol. 96: 127 (2020)

*Chrysozyma sambuci Q.M. Wang, F.Y. Bai \& A.H. Li, Stud. Mycol. 96: 127 (2020)

*Chrysozyma sorbariae Q.M. Wang, F.Y. Bai \& A.H. Li, Stud. Mycol. 96: 129 (2020)

*Ciliosporella italica W.J. Li, Camporesi \& K.D. Hyde, Fungal Diversity 100: 403 (2020)

*Cinereomyces fimbriatus C.L. Zhao, Phytotaxa 459 (1): 55 (2020)

Cintractiella scirpodendri Prychid \& J.J. Bruhl, Fungal Systematics and Evolution 6: 59 (2020)

Circinaria deminuta P.M. McCarthy \& Elix, Australas. Lichenol. 86: 90 (2020)

Circinella lampensis E. Alvarez, C. Muñoz \& I. Fernandez, Persoonia 45: 335 (2020)

Cirrenalia lichenicola Pérez-Ort., Lichenologist 52 (2): 93 (2020)

*Cladocillium musae Chun-Hao Chen \& R. Kirschner, Mycol. Progr. 19 (9): 840 (2020)

Cladophialophora cabanerensis MaciáVicente, Persoonia 44: 371 (2020)

*Cladophialophora nyingchiensis W. Sun, L. Su, M.C. Xiang \& Xing Z. Liu, Journal of Fungi 6 (4, no. 187): 26 (2020)

*Cladophialophora tengchongensis W. Sun, L. Su, M.C. Xiang \& X.Z. Liu, Journal of Fungi 6 (4, no. 187): 27 (2020)

Cladosporium arenosum Gil-Durán \& L. Sanhueza, Persoonia 44: 373 (2020)

Clastoderma confusum K.J. Knight \& Lado, Nuytsia 31: 36 (2020)

Clavaria apulica Agnello \& Papetti, Riv. Micol. 63 (2): 124 (2020)

*Clavariadelphus alpinus J. Zhao \& L.P. Tang, MycoKeys 70: 94 (2020)

*Clavariadelphus amplus J. Zhao, L.P. Tang \& Z.W. Ge, MycoKeys 70: 97 (2020)

*Clavariadelphus aurantiacus P. Zhang,
Mycosystema 39 (9): 1687 (2020)

*Clavariadelphus gansuensis J. Zhao \& L.P. Tang, MycoKeys 70: 104 (2020)

*Clavariadelphus griseoclavus L. Fan \& L. Xia, Phytotaxa 447 (1): 63 (2020)

*Clavariadelphus khinganensis J. Zhao, L.P. Tang \& P. Zhang, MycoKeys 70: 108 (2020)

*Clavariadelphus tenuis P. Zhang, Mycosystema 39 (9): 1688 (2020)

*Claviceps occidentalis M. Liu, Mycologia 112 (5): 984 (2020)

*Claviceps perihumidiphila M. Liu, Mycologia 112 (5): 982 (2020)

*Claviceps quebecensis M. Liu \& J. Cayouette, Mycologia 112 (5): 982 (2020)

*Claviceps ripicola M. Liu, J.G. Menzies \& S.A. Wyka, Mycologia 112 (5): 976 (2020)

*Claviformispora phyllostachydium X.L. Xu \& C.L. Yang, MycoKeys 70: 8 (2020)

Clavispora santaluciensis Drumonde-Neves, Y.R. Domínguez \& Franco-Duarte, Int. J. Syst. Evol. Microbiol. 10.1099/ijsem.0.004531: 5 (2020)

Clavulina sphaeropedunculata E. Pérez-Pazos, M. Villegas-Ríos \& R. Garibay-Orijel, Fungal Diversity 10.1007/s13225-02000461-7: 123 (2020)

Clavulinopsis trigonospora Franchi \& M. Marchetti, Index Fungorum 467: 1 (2020)

*Clitocella orientalis S.P. Jian \& Zhu L. Yang, Mycologia 112 (2): 391 (2020)

Clitocella termitophila T.J. Baroni \& Angelini, Mycol. Progr. 19 (10): 1087 (2020)

*Clitopaxillus dabazi L. Fan \& H. Liu, Mycosystema 39 (9): 1724 (2020)

*Clitopilopsis albida S.P. Jian \& Zhu L. Yang, Mycologia 112 (2): 393 (2020)

*Clitopilus abprunulus S.P. Jian, M. Karadelev \& Zhu L. Yang, Mycol. Progr. 19 (8): 810 (2020)

*Clitopilus brunneiceps S.P. Jian \& Zhu L. Yang, Mycologia 112 (2): 377 (2020)

*Clitopilus rugosiceps S.P. Jian \& Zhu L. 
Yang, Mycologia 112 (2): 379 (2020)

*Clitopilus sinoapalus S.P. Jian \& Zhu L. Yang, Mycologia 112 (2): 385 (2020)

*Clitopilus umbilicatus S.P. Jian \& Zhu L. Yang, Mycologia 112 (2): 386 (2020)

Clitopilus velutinus T.J. Baroni \& Angelini, Mycol. Progr. 19 (10): 1089 (2020)

*Clitopilus yunnanensis S.P. Jian \& Zhu L. Yang, Mycologia 112 (2): 381 (2020)

Clonostachys eriocamporesiana R.H. Perera \& K.D. Hyde, Fungal Diversity 100: 197 (2020)

Clonostachys eriocamporesii R.H. Perera \& K.D. Hyde, Fungal Diversity 100: 199 (2020)

Clonostachys moreaui Lechat, J. Fourn. \& Gasch, Ascomycete.org 12 (2): 35 (2020)

Clonostachys pilosella Lechat \& J. Fourn., Ascomycete.org 12 (3): 61 (2020)

Clonostachys pnagiana Lechat \& J. Fourn., Ascomycete.org 12 (3): 62 (2020)

Clonostachys swieteniae R.H. Perera, E.B.G. Jones \& K.D. Hyde, Mycosphere 11 (1): 2135 (2020)

Clonostachys viticola C. Torcato \& A. Alves, Int. J. Syst. Evol. Microbiol. 10.1099/ijsem.0.004286: 6 (2020)

Codinaea paniculata Réblová \& J. Fourn., MycoKeys 74: 14 (2020)

*Colacogloea aletridis Q.M. Wang, F.Y. Bai \& A.H. Li, Stud. Mycol. 96: 131 (2020)

*Colacogloea hydrangeae Q.M. Wang, F.Y. Bai \& A.H. Li, Stud. Mycol. 96: 132 (2020)

*Colacogloea rhododendri Q.M. Wang, F.Y.

Bai \& A.H. Li, Stud. Mycol. 96: 132 (2020)

Coleophoma eucalyptigena Crous \& R.K. Schumach., Fungal Systematics and Evolution 6: 183 (2020)

Coleophoma podocarpi Crous, Persoonia 44: 325 (2020)

Collemopsidium mauritiae Diederich \& Ertz, Plant and Fungal Systematics 65 (1): 33 (2020)

*Colletotrichum arecicola X.R. Cao, H.Y. Che
\& D.Q. Luo, Pl. Dis. 104 (5): 1371 (2020)

*Colletotrichum citrus-medicae Qian Zhang, Yong Wang bis, Jayawardena \& K.D. Hyde, Fungal Diversity 10.1007/s13225-02000458-2: 23 (2020)

Colletotrichum cycadis Andjic, A. Maxwell \& K. Sm., Persoonia 45: 337 (2020)

*Colletotrichum eriobotryae Damm \& C.J. Huang, Mycol. Progr. 19 (4): 373 (2020)

*Colletotrichum hainanense Wu Zhang \& X.L. Niu, Pl. Dis. 104 (6): 1747 (2020)

Colletotrichum hederiicola Jayaward. Camporesi \& K.D. Hyde, Fungal Diversity 100: 193 (2020)

*Colletotrichum miaoliense P.C. Chung \& H.Y. Wu, Scientific Reports 10 (no. 14664): 6 (2020)

*Colletotrichum neorubicola $\mathrm{Yu} \mathrm{Li,} \mathrm{J.} \mathrm{Gao} \mathrm{\&}$ L.P. Liu, Mycol. Progr. 19 (9): 951 (2020)

Colletotrichum orchidis Jayaward., Camporesi \& K.D. Hyde, Mycosphere 11 (1): 595 (2020) Colletotrichum parthenocissicola Jayawardena, Bulgakov, Huanraleuk \& K.D. Hyde, Fungal Diversity 10.1007/s13225020-00461-7: 88 (2020)

*Collodiscula tubulosa Q.R. Li, J.C. Kang \& K.D. Hyde, Phytotaxa 428 (2): 125 (2020)

Coltricia robusta Vlasák \& Ryvarden, Syn. Fung. 42: 30 (2020)

*Coltricia subcinnamomea L.S. Bian \& Y.C. Dai, Mycol. Progr. 19 (7): 660 (2020)

*Coltricia subverrucata L.S. Bian \& Y.C. Dai, Mycol. Progr. 19 (7): 662 (2020)

Comoclathris europaeae Brahmanage, Camporesi \& K.D. Hyde, Mycosphere 11: 2519 (2020)

Comoclathris flammulae Brahmanage, Camporesi \& K.D. Hyde, Mycosphere 11: 2523 (2020)

Comoclathris galatellae D. Pem, T.S. Bulgakov \& K.D. Hyde, Mycosphere 11 (1): 1983 (2020)

Comoclathris lonicerae Brahmanage, 
Camporesi \& K.D. Hyde, Mycosphere 11: 2525 (2020)

*Conidiobolus bifurcatus B. Huang \& Y. Nie, MycoKeys 73: 139 (2020)

Conidiobolus lunulus D. Goffre, R.A. Humber \& P.J. Folgarait, Mycologia 10.1080/00275514.2020.1816387: 4 (2020)

*Conidiobolus taihushanensis B. Huang \& Y. Nie, MycoKeys 73: 141 (2020)

*Conidiobolus variabilis B. Huang \& Y. Nie, Mycologia 73: 143 (2020)

Conidiocarpus guilanensis Khodap., Mycol. Progr. 19 (2): 161 (2020)

Coniochaeta arenariae Dayarathne \& E.B.G. Jones, Mycosphere 11 (1): 127 (2020)

Coniochaeta krabiensis Dayarathne \& E.B.G. Jones, Mycosphere 11 (1): 128 (2020)

*Coniochaeta vineae S.K. Huang, T.C. Wen \& K.D. Hyde, Fungal Diversity 100: 211 (2020)

*Conioscypha verrucosa J. Yang \& K.D. Hyde, Fungal Diversity 100: 205 (2020)

Coniothyrium triseptatum Dayarathne, Thyagaraja \& K.D. Hyde, Fungal Diversity 10.1007/s13225-020-00461-7: 16 (2020)

Coprinellus ovatus M. Kamran \& S. Jabeen, Mycotaxon 135 (6): 326 (2020)

Coprinopsis paracinerea Bender \& A. Melzer, Z. Mykol. 87 (1): 36 (2020)

Coprinopsis pernambucensis Voto, Boll. Assoc. Micol. Ecol. Romana 109 (1): 12 (2020)

Coprinopsis rubra Örstadius, E. Larss. \& L. Nagy, Persoonia 45: 339 (2020)

Coprinopsis siepei Bender \& A. Melzer, Z. Mykol. 87 (1): 40 (2020)

Cora timucua Dal Forno, Kaminsky \& Lücking, Bryologist 123 (4): 664 (2020)

Cordyceps araneae Mongkols., Tasan., Noisrip., Himaman \& Luangsa-ard, Mycol. Progr. 19 (9): 972 (2020)

Cordyceps brevistroma Mongkols., Tasan., Thanakitp. \& Luangsa-ard, Mycol. Progr. 19 (9): 972 (2020)
*Cordyceps chaetoclavata H. Yu, Y.B. Wang, Y. Wang, Q. Fan \& Zhu L. Yang, Fungal Diversity 103: 14 (2020)

*Cordyceps cocoonihabita H. Yu, Y.B. Wang, Y. Wang, Q. Fan \& Zhu L. Yang, Fungal Diversity 103: 15 (2020)

Cordyceps inthanonensis Mongkols., Tasan., Thanakitp. \& Luangsa-ard, Mycol. Progr. 19 (9): 973 (2020)

Cordyceps neopruinosa Mongkols., Noisrip., Khons. \& Luangsa-ard, Mycol. Progr. 19 (9): 976 (2020)

Cordyceps parvistroma Mongkols., Tasan., Thanakitp. \& Luangsa-ard, Mycol. Progr. 19 (9): 976 (2020)

*Cordyceps shuifuensis H. Yu, Y.B. Wang, Y. Wang \& Zhu L. Yang, Fungal Diversity 103: 17 (2020)

*Cordyceps subtenuipes H. Yu, Y.B. Wang, Y. Wang, D.E. Duan \& Zhu L. Yang, Fungal Diversity 103: 17 (2020)

*Cordyceps yinjiangensis Y.P. Li, W.H. Chen, Y.F. Han \& Z.Q. Liang, Phytotaxa 453 (3): 286 (2020)

Corethromyces gibbosus W. Rossi \& Santam., Cryptog. Mycol. 41 (4): 71 (2020)

Corethromyces marshallii W. Rossi \& Santam., Cryptog. Mycol. 41 (4): 72 (2020)

*Corniculariella rhamni W.J. Li, Camporesi \& K.D. Hyde, Fungal Diversity 100: 420 (2020)

Corticifraga nephromatis Pérez-Ort., Lichenologist 52 (2): 93 (2020)

Cortinarius afropurpurissimus C. Sharp, Syn. Fung. 40: 8 (2020)

Cortinarius ainsworthii Liimat. \& Niskanen, Fungal Diversity 100: 244 (2020)

Cortinarius aurae Niskanen \& Liimat., Fungal Diversity 100: 247 (2020)

Cortinarius azureolamellatus C. Sharp, Syn. Fung. 40: 21 (2020)

Cortinarius brevirobustus C. Sharp, Syn. Fung. 40: 23 (2020) 
Cortinarius britannicus Liimat. \& Niskanen, Fungal Diversity 100: 247 (2020)

Cortinarius bubulus Liimat., Kytov. \& Niskanen, Index Fungorum 438: 5 (2020)

Cortinarius chlorosplendidus Furci, Niskanen, San-Fabian, Liimat. \& Salgado Salomón, Mycologia 10.1080/00275514.2019.1689763: 7 (2020)

Cortinarius clarisordidus Niskanen, Kytov. \& Liimat., Index Fungorum 438: 2 (2020)

Cortinarius diaphorus Soop, A.R. Nilsen \& Orlovich, Mycologia 112 (3): 594 (2020)

Cortinarius fibrillosibrunneus Kytov., Niskanen \& Liimat., Index Fungorum 438: 3 (2020)

Cortinarius flammeouraceus Niskanen, Kytov., Liimat., Ammirati \& Dima, Index Fungorum 438: 5 (2020)

Cortinarius glaucoelotus Brandrud, Dima, Krisai, Ballarà \& Peintner, Persoonia 45: 405 (2020)

Cortinarius heatherae Overall, Fungal Diversity 100: 249 (2020)

Cortinarius hepaticus Kytov., Niskanen \& Liimat., Index Fungorum 438: 3 (2020)

Cortinarius indopurpurascens Dima, Semwal, Brandrud, V. Papp \& V.K. Bhatt, Persoonia 45: 403 (2020)

Cortinarius indorusseus Dima, Semwal, V.K. Bhatt \& Brandrud, Fungal Diversity 10.1007/s13225-020-00461-7: 107 (2020)

*Cortinarius laccariphyllus Y. Li \& M.L. Xie, MycoKeys 69: 97 (2020)

Cortinarius lentus Boccardo, Cleric., Dovana \& Vizzini, Phytotaxa 447 (1): 35 (2020)

Cortinarius lindstroemii Niskanen, Kytov. \& Liimat., Index Fungorum 438: 1 (2020)

Cortinarius mallaensis Kytov., Niskanen \& Liimat., Index Fungorum 438: 4 (2020)

*Cortinarius minorisporus X. Yue Wang, J.A. Cooper, Mycologia 112 (3): 595 (2020)

Cortinarius mtaoensis C. Sharp, Syn. Fung. 40: 31 (2020)
Cortinarius mukuvusiensis C. Sharp \& B. Dima, Syn. Fung. 40: 34 (2020)

*Cortinarius neotorvus Y. Li, M.L. Xie \& T.Z. Wei, MycoKeys 69: 99 (2020)

Cortinarius occidentalisagacitas Liimat., Niskanen, Kytov. \& Ammirati, Index Fungorum 438: 6 (2020)

Cortinarius odoritraganus Niskanen, Liimat. \& Ammirati, Index Fungorum 438: 6 (2020)

Cortinarius olivaceovaginatus Niskanen, SanFabian, Liimat. \& E. Horak, Mycologia 10.1080/00275514.2019.1689763: 9 (2020)

Cortinarius paezii Garrido-Benavent, Ballarà, Liimat. \& Mahiques, Persoonia 44: 379 (2020)

Cortinarius pakistanicus A. Naseer \& A.N. Khalid, MycoKeys 74: 99 (2020)

Cortinarius pallidibrunneus Niskanen, Kytov. \& Liimat., Index Fungorum 438: 2 (2020)

Cortinarius paraxenosma Soop \& Dima, Phytotaxa 438 (4): 231 (2020)

Cortinarius paululus Kokkonen, Karstenia 58 (1): 88 (2020)

Cortinarius paulus Kokkonen, Karstenia 58 (1): 90 (2020)

Cortinarius paurigarhwalensis Semwal, Dima \& Soop, Fungal Diversity 10.1007/s13225020-00461-7: 108 (2020)

Cortinarius pinisquamulosus Kytov., Niskanen \& Liimat., Index Fungorum 438: 1 (2020)

Cortinarius pseudotorvus A. Naseer, J. Khan \& A.N. Khalid, MycoKeys 74: 101 (2020)

*Cortinarius purpureocapitatus X. Yue Wang, J.A. Cooper, A.R. Nilsen \& Orlovich, Mycologia 112 (3): 596 (2020)

Cortinarius rusticelloides Kokkonen, Karstenia 58 (1): 94 (2020)

Cortinarius sagacitas Kytov., Niskanen \& Liimat., Index Fungorum 438: 7 (2020)

Cortinarius sagarum Kokkonen, Karstenia 58 (1): 68 (2020)

Cortinarius scabridipileus Kytov., Liimat. \& 
Niskanen, Index Fungorum 438: 7 (2020)

Cortinarius scoticus Niskanen \& Liimat., Fungal Diversity 100: 251 (2020)

*Cortinarius sinensis L.H. Sun, T.Z. Wei \& Y.J. Yao, Fungal Diversity 104: 109 (2020)

Cortinarius subcosmoxanthus Liimat., SanFabian \& Niskanen, Mycologia 10.1080/00275514.2019.1689763: 9 (2020)

*Cortinarius subfuscoperonatus Y. Li \& M.L.

Xie, MycoKeys 69: 101 (2020)

*Cortinarius subsanguineus T.Z. Wei, M.L.

Xie \& Y.J. Yao, Fungal Diversity 104: 112 (2020)

Cortinarius subsaniosus Liimat. \& Niskanen, Fungal Diversity 100: 252 (2020)

Cortinarius tiffanyae Healy, Ammirati \& Liimat., Index Fungorum 456: 1 (2020)

Cortinarius vienoi Kokkonen, Karstenia 58 (1): 99 (2020)

Cortinarius violaceocystidiatus A.R. Nilsen \& Orlovich, Mycologia 112 (3): 597 (2020)

*Cortinarius xiaojinensis T.Z. Wei, M.L. Xie \& Y.J. Yao, Fungal Diversity 104: 115 (2020)

*Corylicola italica Wijesinghe, Camporesi, Yong Wang bis \& K.D. Hyde, Biodiversity Data Journal 8 (e55957): 8 (2020)

*Corynespora doipuiensis J.F. Li \& Phookamsak, Asian Journal of Mycology 3 (1): 62 (2020)

*Corynespora lignicola Z.L. Luo, H.Y. Su \& K.D. Hyde, Asian Journal of Mycology 3 (1): 65 (2020)

*Corynespora sinensis Jian Ma, X.G. Zhang \& R.F. Castañeda, Mycotaxon 135 (4): 804 (2020)

*Corynespora submersa Z.L. Luo, H.Y. Su \& K.D. Hyde, Asian Journal of Mycology 3 (1): 63 (2020)

*Corynesporopsis hainanensis Z.H. Xu, Jian Ma, X.G. Zhang \& R.F. Castañeda, Mycotaxon 135 (3): 624 (2020)

Cratiria jamesiana Elix \& H. Mayrhofer, Australas. Lichenol. 86: 87 (2020)
*Crepidotus dentatus T. Bau \& Y.P. Ge, Mycosystema 39 (2): 246 (2020)

Crepidotus exilis A.M. Kumar \& C.K. Pradeep, Aust. Syst. Bot. 33: 382 (2020)

Crepidotus globisporus A.M. Kumar \& C.K. Pradeep, Aust. Syst. Bot. 33: 386 (2020)

*Crepidotus heterocystidiosus T. Bau \& Y.P. Ge, Mycosystema 39 (2): 246 (2020)

*Crepidotus pseudomollis T. Bau \& Y.P. Ge, Mycosystema 39 (2): 248 (2020)

*Crepidotus reticulatus T. Bau \& Y.P. Ge, Mycosystema 39 (2): 249 (2020)

*Crepidotus striatus T. Bau \& Y.P. Ge, Mycosystema 39 (2): 251 (2020)

*Crepidotus trichocraspedotus T. Bau \& Y.P. Ge, Mycosystema 39 (2): 252 (2020)

Cresponea graemeannae Kantvilas, Lichenologist 52 (4): 281 (2020)

Cribraria bicolor S.L. Stephenson, Novozh. \& P. Wellman, Nov. sist. Niz. Rast. 54 (2): 381 (2020)

Crinipellis nigrolamellata Antonín, Fiard, Ševčíková, Dumez \& Courtec., Persoonia 45: 341 (2020)

Cryolevonia giraudoae $\mathrm{V}$. de Garcia, Trochine \& Libkind, Int. J. Syst. Evol. Microbiol. 70 (6): 3715 (2020)

Cryolevonia schafbergensis A. Pontes, Ruethi, B. Frey, A. Aires, A. Thomas, D. Overy, B. Halti, R. Kerr \& J.P. Sampaio, Int. J. Syst. Evol. Microbiol. 10.1099/ijsem.0.004040: 3 (2020)

*Cryphognomonia pini C.M. Tian \& N. Jiang, MycoKeys 69: 81 (2020)

Cryptodiscus gassicurtiae Etayo \& Aptroot, Bryologist 123 (3): 486 (2020)

Cryptosphaeria avicenniae Devadatha \& V.V. Sarma, Cryptog. Mycol. 41 (3): 34 (2020)

Cryptosphaeria halophila Dayar. \& K.D. Hyde, Cryptog. Mycol. 41 (3): 40 (2020)

Ctenomyces indicus Rahul Sharma \& Shouche, Mycopathologia 185 (1): 79 (2020)

Cuphophyllus esteriae Voitk, I. Saar \& E. 
Larss., Mycologia 112 (2): 447 (2020)

Cuphophyllus lamarum Voitk, Boertm. \& I. Saar, Mycologia 112 (2): 449 (2020)

Curvularia arcana Hern.-Restr. \& Y. Marín, Mycol. Progr. 19 (6): 564 (2020)

Curvularia austriaca Y. Marín \& Crous, Mycol. Progr. 19 (6): 564 (2020)

Curvularia canadensis Y. Marín \& Crous, Mycol. Progr. 19 (6): 569 (2020)

Curvularia coimbatorensis N. Kiss \& Kocsubé, Pathogens 9 (1, no. 9): 7 (2020)

Curvularia ellisii Y. Marín \& Crous, Mycol. Progr. 19 (6): 572 (2020)

Curvularia iranica Mehrabi-Koushki, Safi \& Farokhinejad, Mycol. Progr. 19 (9): 942 (2020)

Curvularia moringae Crous, Persoonia 45: 323 (2020)

*Curvularia nanningensis Qian Zhang, K.D. Hyde \& Yong Wang bis, MycoKeys 63: 58 (2020)

Curvularia paraverruculosa IturrietaGonzález, Gené \& Dania García, MycoKeys 68: 11 (2020)

Curvularia pseudoclavata Y. Marín \& Crous, Mycol. Progr. 19 (6): 578 (2020)

Curvularia pseudoellisii Y. Marín \& Crous, Mycol. Progr. 19 (6): 579 (2020)

Curvularia pseudointermedia Y. Marín \& Crous, Mycol. Progr. 19 (6): 580 (2020)

Curvularia pseudoprotuberata Y. Marín \& Crous, Mycol. Progr. 19 (6): 581 (2020)

Curvularia siddiquii Y. Marín \& Crous, Mycol. Progr. 19 (6): 582 (2020)

Curvularia suttoniae Iturrieta-González, Wiederhold, Gené \& Dania García, MycoKeys 68: 13 (2020)

Curvularia tamilnaduensis N. Kiss \& Kocsubé, Pathogens 9 (1, no. 9): 8 (2020)

Curvularia tribuli Hern.-Restr. \& Y. Marín, Mycol. Progr. 19 (6): 584 (2020)

Curvularia vietnamensis Iturrieta-González, Gené \& Dania García, MycoKeys 68: 14
(2020)

Cutaneotrichosporon cavernicola M. Takash., Kurakado, O. Cho, K. Kikuchi, Sugiy. \& Sugita, Int. J. Syst. Evol. Microbiol. 10.1099/ijsem.0.004277: 10 (2020)

Cutaneotrichosporon middelhovenii $\mathrm{M}$. Takash., Kurakado, O. Cho, K. Kikuchi, Sugiy. \& Sugita, Int. J. Syst. Evol. Microbiol. 10.1099/ijsem.0.004277: 10 (2020)

Cyathus villosocarpus J.S. Góis, R. Cruz, P.H.G. Nascim. \& Baseia, N.Z. Jl Bot. 10.1080/0028825X.2020.1757469: 3 (2020)

Cyberlindnera dauci A.M. Glushakova, M.A.

Tomashevskaya \& Kachalkin, Persoonia 45: 343 (2020)

Cylindrium corymbiae Crous, Fungal Systematics and Evolution 6: 184 (2020)

Cylindrium magnoliae A. Pintos, M. González, P. Alvarado \& E. Rubio, Persoonia 44: 381 (2020)

Cylindromonium alloxyli Crous, Persoonia 45: 281 (2020)

Cyphellophora vietnamensis IturrietaGonzález, Dania García, Guarro \& Gené, Persoonia 45: 345 (2020)

Cystobasidium alpinum Turchetti, Selbmann, Onofri \& Buzzini, Stud. Mycol. 96: 133 (2020)

*Cystobasidium raffinophilum Q.M. Wang, F.Y. Bai \& A.H. Li, Stud. Mycol. 96: 116 (2020)

*Cystobasidium terricola Q.M. Wang, F.Y. Bai \& A.H. Li, Stud. Mycol. 96: 116 (2020) Cystomyces antheropori Ayawong \& Y. Ono, Mycoscience 61 (4): 180 (2020)

*Cytospora cinnamomea M. Pan \& X.L. Fan, Frontiers in Plant Science 11 (no. 690): 4 (2020)

*Cytospora coryli H.Y. Zhu \& X.L. Fan, MycoKeys 62: 110 (2020)

*Cytospora cotoneastricola M. Pan \& X.L. Fan, Frontiers in Plant Science 11 (no. 690): 5 (2020) 
*Cytospora diopuiensis Q.J. Shang, J.K. Liu \& K.D. Hyde, Mycosphere 11 (1): 202 (2020)

Cytospora fusispora M. Niranjan \& V.V. Sarma, Fungal Diversity 100: 167 (2020)

*Cytospora galegicola Q.J. Shang, E. Camporesi \& K.D. Hyde, Mycosphere 11 (1): 206 (2020)

*Cytospora globosa W.J. Li, Camporesi \& K.D. Hyde, Fungal Diversity 100: 447 (2020)

*Cytospora kuanchengensis C.M. Tian \& N. Jiang, MycoKeys 62: 14 (2020)

*Cytospora mali-spectabilis M. Pan \& X.L. Fan, Frontiers in Plant Science 11 (no. 690): 7 (2020)

*Cytospora ochracea M. Pan \& X.L. Fan, Frontiers in Plant Science 11 (no. 690): 8 (2020)

*Cytospora olivacea M. Pan \& X.L. Fan, Frontiers in Plant Science 11 (no. 690): 9 (2020)

*Cytospora phialidica W.J. Li, Camporesi \& K.D. Hyde, Fungal Diversity 100: 447 (2020) *Cytospora pingbianensis Q.J. Shang, K.D. Hyde \& J.K. Liu, Mycosphere 11 (1): 206 (2020)

*Cytospora pruni-mume M. Pan \& X.L. Fan, Frontiers in Plant Science 11 (no. 690): 12 (2020)

*Cytospora pubescentis Q.J. Shang, E. Camporesi \& K.D. Hyde, Mycosphere 11 (1): 213 (2020)

*Cytospora rosicola M. Pan \& X.L. Fan, Frontiers in Plant Science 11 (no. 690): 13 (2020)

Cytospora rosigena Chaiwan, Wanas., Bulgakov \& K.D. Hyde, Fungal Diversity 100: 174 (2020)

*Cytospora sorbina M. Pan \& X.L. Fan, Frontiers in Plant Science 11 (no. 690): 13 (2020)

*Cytospora spiraeicola H.Y. Zhu \& X.L. Fan, MycoKeys 62: 115 (2020)

*Cytospora tibetensis M. Pan \& X.L. Fan,
Frontiers in Plant Science 11 (no. 690): 13 (2020)

*Cytospora xinglongensis C.M. Tian \& N. Jiang, MycoKeys 62: 18 (2020)

*Cytospora xinjiangensis M. Pan \& X.L. Fan, Frontiers in Plant Science 11 (no. 690): 14 (2020)

Dacryonaema macrosporum J.C. Zamora \& S. Ekman, Persoonia 44: 194 (2020)

Dactylonectria riojana Berlanas, AndrésSodupe, Ojeda \& Gramaje, Pl. Dis. 104 (1): 98 (2020)

Daldinia chiangdaoensis Srikit., Wongkan., M. Stadler \& Luangsa-ard, Mycol. Progr. 19 (10): 1120 (2020)

Daldinia flavogranulata Srikit., Wongkan., M. Stadler \& Luangsa-ard, Mycol. Progr. 19 (10): 1125 (2020)

Daldinia phadaengensis Srikit., Wongkan., M. Stadler \& Luangsa-ard, Mycol. Progr. 19 (10): 1124 (2020)

Davidhawksworthia quintiniae Crous, Persoonia 45: 279 (2020)

Deconica esperancensis Ram.-Cruz \& Glez.Adame, Mycol. Progr. 19 (11): 1322 (2020)

Deconica milvispora Ram.-Cruz \& Matheny, Mycol. Progr. 19 (11): 1322 (2020)

*Dendrographium multiseptatum L.G. Ma \& J.S. Qi, Mycotaxon 134 (4): 634 (2020)

Dendrostoma luteum L.A. Shuttlew., A.J. Lewis, C. Gorton \& Pérez-Sierra, Persoonia 44: 383 (2020)

*Dendryphion hydei J.F. Li, Phookamsak \& Jeewon, PLoS ONE 15 (2): e0228067, 6 (2020)

*Derxomyces bifurcus Q.M. Wang, F.Y. Bai \& A.H. Li, Stud. Mycol. 96: 95 (2020)

*Derxomyces cylindricus F.Y. Bai, Q.M. Wang \& M. Takash. ex F.Y. Bai \& Q.M. Wang, Stud. Mycol. 96: 133 (2020)

*Derxomyces elongatus Q.M. Wang, F.Y. Bai \& A.H. Li, Stud. Mycol. 96: 95 (2020)

*Derxomyces hubeiensis F.Y. Bai, Q.M. 
Wang \& M. Takash. ex F.Y. Bai \& Q.M. Wang, Stud. Mycol. 96: 133 (2020)

*Derxomyces longicylindricus Q.M. Wang, F.Y. Bai \& A.H. Li, Stud. Mycol. 96: 98 (2020)

*Derxomyces longiovatus Q.M. Wang, F.Y. Bai \& A.H. Li, Stud. Mycol. 96: 94 (2020)

*Derxomyces melastomatis Q.M. Wang, F.Y. Bai \& A.H. Li, Stud. Mycol. 96: 97 (2020)

*Derxomyces nakasei F.Y. Bai, Q.M. Wang \& M. Takash. ex F.Y. Bai \& Q.M. Wang, Stud. Mycol. 96: 133 (2020)

*Derxomyces napiformis Q.M. Wang, F.Y. Bai \& A.H. Li, Stud. Mycol. 96: 95 (2020)

*Derxomyces ovatus Q.M. Wang, F.Y. Bai \& A.H. Li, Stud. Mycol. 96: 98 (2020)

*Derxomyces polymorphus Q.M. Wang, F.Y. Bai \& A.H. Li, Stud. Mycol. 96: 93 (2020)

*Derxomyces pseudoboekhoutii Q.M. Wang, F.Y. Bai \& A.H. Li, Stud. Mycol. 96: 93 (2020)

*Derxomyces pseudoyunnanensis Q.M. Wang, F.Y. Bai \& A.H. Li, Stud. Mycol. 96: 94 (2020)

*Derxomyces taiwanicus Q.M. Wang, F.Y. Bai \& A.H. Li, Stud. Mycol. 96: 97 (2020)

*Derxomyces xingshanicus Q.M. Wang, F.Y. Bai \& A.H. Li, Stud. Mycol. 96: 94 (2020)

Diabolocovidia claustri Crous, Persoonia 44: 331 (2020)

*Diaporthe acuta Y.S. Guo \& G.P. Wang, Persoonia 45: 140 (2020)

*Diaporthe albosinensis C.M. Tian \& Q. Yang, MycoKeys 67: 9 (2020)

Diaporthe araucanorum M. Zapata, M.A. Palma, M.J. Anninat \& E. Piontelli, Int. J. Syst. Evol. Microbiol. 10.1099/ijsem.0.004183: 6 (2020)

*Diaporthe arezzoensis W.J. Li, Camporesi \& K.D. Hyde, Fungal Diversity 100: 472 (2020)

*Diaporthe australiana R.G. Shivas, Akinsanmi \& Y.P. Tan, Pl. Path. 69 (5): 916 (2020)
Diaporthe camporesii Manawasinghe \& K.D. Hyde, Fungal Diversity 100: 177 (2020)

*Diaporthe chongqingensis Y.S. Guo \& G.P. Wang, Persoonia 45: 146 (2020)

Diaporthe chromolaenae Mapook \& K.D. Hyde, Fungal Diversity 101: 130 (2020)

Diaporthe clematidina Phukhams., M.V. de Bult \& K.D. Hyde, Fungal Diversity 10.1007/s13225-020-00448-4: 160 (2020)

*Diaporthe constrictospora Y.Y. Chen, A.J. Dissanayake \& Jian K. Liu, Journal of Fungi 6 (no. 251): 14 (2020)

*Diaporthe coryli C.M. Tian \& Q. Yang, MycoKeys 67: 11 (2020)

Diaporthe crousii S. Hilário, L. Santos \& A. Alves, Mycologia 112 (2): 299 (2020)

Diaporthe delonicis R.H. Perera, E.B.G. Jones \& K.D. Hyde, Mycosphere 11 (1): 2129 (2020)

*Diaporthe drenthii Y.P. Tan, Akinsanmi \& R.G. Shivas, Pl. Path. 69 (5): 916 (2020)

Diaporthe durionigena L.D. Thao, L.T. Hien, N.V. Liem, H.M. Thanh \& T.N. Khanh, Persoonia 44: 385 (2020)

*Diaporthe ellipsospora Y.Y. Chen, A.J. Dissanayake \& Jian K. Liu, Journal of Fungi 6 (no. 251): 17 (2020)

Diaporthe foikelawen M. Zapata, M.A. Palma, M.J. Anninat \& E. Piontelli, Int. J. Syst. Evol. Microbiol. 10.1099/ijsem.0.004183: 7 (2020)

*Diaporthe fulvicolor Y.S. Guo \& G.P. Wang, Persoonia 45: 146 (2020)

*Diaporthe guttulata Y.Y. Chen, A.J. Dissanayake \& Jian K. Liu, Journal of Fungi 6 (no. 251): 19 (2020)

*Diaporthe irregularis Y.Y. Chen, A.J. Dissanayake \& Jian K. Liu, Journal of Fungi 6 (no. 251): 20 (2020)

*Diaporthe lenispora Y.Y. Chen, A.J. Dissanayake \& Jian K. Liu, Journal of Fungi 6 (no. 251): 20 (2020)

*Diaporthe macadamiae Y.P. Tan, Akinsanmi \& R.G. Shivas, Pl. Path. 69 (5): 916 (2020) 
Diaporthe marina Dayarathne, Mycosphere 11 (1): 99 (2020)

Diaporthe mediterranea M. León, RodríguezReina \& Armengol, Agronomy 10 (8, no. 1062): 17 (2020)

*Diaporthe minima Y.Y. Chen, A.J. Dissanayake \& Jian K. Liu, Journal of Fungi 6 (no. 251): 22 (2020)

*Diaporthe minusculata Y.Y. Chen, A.J. Dissanayake \& Jian K. Liu, Journal of Fungi 6 (no. 251): 22 (2020)

Diaporthe nigra Brahmanage \& K.D. Hyde, Fungal Diversity 100: 185 (2020)

*Diaporthe parva Y.S. Guo \& G.P. Wang, Persoonia 45: 154 (2020)

Diaporthe patagonica M. Zapata, M.A. Palma, M.J. Anninat \& E. Piontelli, Int. J. Syst. Evol. Microbiol. 10.1099/ijsem.0.004183: 9 (2020)

Diaporthe phillipsii S. Hilário, L. Santos \& A. Alves, Mycologia 112 (2): 301 (2020)

Diaporthe pimpinellae Abeywickrama, Camporesi, Dissanayake \& K.D. Hyde, Fungal Diversity 10.1007/s13225-02000461-7: 77 (2020)

Diaporthe rosiphthora C.M. Pereira, B.W. Ferreira \& R.W. Barreto, Crop Protection 139 (no. 105365): 6 (2020)

Diaporthe rossmaniae S. Hilário, I. Amaral, L. Santos \& A. Alves, Mycologia 112 (2): 302 (2020)

*Diaporthe searlei R.G. Shivas, Akinsanmi \& Y.P. Tan, Pl. Path. 69 (5): 918 (2020)

*Diaporthe shaanxiensis C.M. Tian \& Q. Yang, MycoKeys 67: 13 (2020)

*Diaporthe spinosa Y.S. Guo \& G.P. Wang, Persoonia 45: 154 (2020)

Diaporthe tarchonanthi Crous, Fungal Systematics and Evolution 6: 185 (2020)

Diaporthe vacuae S. Hilário, L. Santos \& A. Alves, Mycologia 112 (2): 303 (2020)

*Diaporthe zaobaisu Y.S. Guo \& G.P. Wang, Persoonia 45: 156 (2020)

Diatrype mangrovei Dayar. \& K.D. Hyde,
Cryptog. Mycol. 41 (3): 46 (2020)

Diatrypella elaeidis Konta \& K.D. Hyde, Mycosphere 11 (1): 256 (2020)

Diatrypella yunnanensis Brahamanage, Thyagaraja \& K.D. Hyde, Fungal Diversity 100: 226 (2020)

Dictyoarthrinium musae Samarakoon, Chomnunti \& K.D. Hyde, MycoKeys 71: 109 (2020)

Dictyochaeta coryli R.H. Perera, E.B.G. Jones \& K.D. Hyde, Mycosphere 11 (1): 2120 (2020)

Dictyochaeta lithocarpi R.H. Perera, E.B.G. Jones \& K.D. Hyde, Mycosphere 11 (1): 2124 (2020)

Dictyocheirospora clematidis Phukhams., D.J. Bhat \& K.D. Hyde, Fungal Diversity 10.1007/s13225-020-00448-4: 17 (2020)

*Dictyocheirospora thailandica X.D. Yu, W. Dong \& H. Zhang, Fungal Diversity 10.1007/s13225-020-00463-5: 82 (2020)

*Dictyosporella guizhouensis J. Yang \& K.D. Hyde, Fungal Diversity 10.1007/s13225020-00461-7: 93 (2020)

Dictyosporium marinum Dayarathne \& E.B.G. Jones, Mycosphere 11 (1): 33 (2020)

*Dictyosporium muriformis N.G. Liu, K.D. Hyde \& J.K. Liu, Fungal Diversity 100: 33 (2020)

*Didymella aloeicola L.W. Hou, L. Cai \& Crous, Stud. Mycol. 96: 352 (2020)

Didymella camporesii Manawasinghe \& K.D. Hyde, Fungal Diversity 100: 38 (2020)

Didymella chromolaenae Mapook \& K.D. Hyde, Fungal Diversity 101: 16 (2020)

Didymella corylicola Voglmayr, Scarpari, Di Giambattista, Vitale \& Luongo, Mycol. Progr. 19 (4): 323 (2020)

*Didymella degraaffiae Hern.-Restr., L.W. Hou, L. Cai \& Crous, MycoKeys 65: 73 (2020)

*Didymella guttulata L.W. Hou, L. Cai \& Crous, Stud. Mycol. 96: 353 (2020) 
*Didymella indica L.W. Hou, L. Cai \& Crous, Stud. Mycol. 96: 355 (2020)

*Didymella kooimaniorum Hern.-Restr., L.W. Hou, L. Cai \& Crous, MycoKeys 65: 74 (2020)

*Didymella mitis L.W. Hou, L. Cai \& Crous, Stud. Mycol. 96: 356 (2020)

*Didymella prolaticolla L.W. Hou, L. Cai \& Crous, Stud. Mycol. 96: 357 (2020)

*Didymella subglobispora L.W. Hou, L. Cai \& Crous, Stud. Mycol. 96: 359 (2020)

*Didymella subrosea L.W. Hou, L. Cai \& Crous, Stud. Mycol. 96: 361 (2020)

*Didymella variabilis L.W. Hou, L. Cai \& Crous, Stud. Mycol. 96: 362 (2020)

*Digitothyrea subdivergens X.L. Wei, Mycosystema 39 (4): 3 (2020)

Diorygma cameroonense Kalb, Archive For Lichenology 15: 4 (2020)

Diorygma isidiolichexanthonicum Aptroot, Archive For Lichenology 16: 5 (2020)

*Dioszegia heilongjiangensis Q.M. Wang, F.Y. Bai \& A.H. Li, Stud. Mycol. 96: 89 (2020)

*Dioszegia kandeliae Q.M. Wang, F.Y. Bai, L.D. Guo \& A.H. Li, Stud. Mycol. 96: 90 (2020)

*Dioszegia maotaiensis Q.M. Wang, F.Y. Bai \& A.H. Li, Stud. Mycol. 96: 90 (2020)

*Dioszegia milinica Q.M. Wang, F.Y. Bai \& A.H. Li, Stud. Mycol. 96: 89 (2020)

*Dioszegia ovata Q.M. Wang, F.Y. Bai \& A.H. Li, Stud. Mycol. 96: 89 (2020)

*Dioszegia zsoltii F.Y. Bai, M. Takash. \& Nakase, Stud. Mycol. 96: 133 (2020)

Diphymyces torresii W. Rossi \& Santam., Cryptog. Mycol. 41 (4): 73 (2020)

Diplodia torilicola Harishchandra, Camporesi, A.J.L. Phillips \& K.D. Hyde, Fungal Diversity 10.1007/s13225-020-00461-7: 11 (2020)

Diplomitoporus densiporus Decock \& Ryvarden, Syn. Fung. 42: 8 (2020)
Diplomitoporus gabonensis Decock \& Ryvarden, Syn. Fung. 42: 9 (2020)

Diplomitoporus minutoporus Ryvarden, Syn. Fung. 40: 102 (2020)

Diplomitoporus nigrus Ryvarden, Syn. Fung. 40: 102 (2020)

Dirinaria hypoleuca Kalb, Archive For Lichenology 18: 3 (2020)

*Distoseptispora bambusae Y.R. Sun, I.D. Goonasekara, Yong. Wang bis \& K.D. Hyde, Biodiversity Data Journal 8 (e53678): 6 (2020)

Distoseptispora clematidis Phukhams., M.V. de Bult \& K.D. Hyde, Fungal Diversity 10.1007/s13225-020-00448-4: 168 (2020)

Distoseptispora hydei Monkai \& Phookamsak, Phytotaxa 459 (2): 97 (2020)

*Distoseptispora longispora H.Y. Song \& D.M. Hu, Mycotaxon 135 (3): 516 (2020)

*Distoseptispora rayongensis J. Yang \& K.D. Hyde, Mycosphere 11 (1): 579 (2020)

Diutina bernalii Haelew., Pfliegler, Horváth \& Imre, Fungal Diversity 100: 239 (2020)

Diutina sipiczkii Pfliegler, Haelew., Horváth \& Imre, Fungal Diversity 100: 239 (2020)

Dothiora aloidendri Crous, Persoonia 45: 297 (2020)

Dothiorella ostryae Manawasinghe, Camporesi \& K.D. Hyde, Fungal Diversity 10.1007/s13225-020-00462-6: 37 (2020)

Dyfrolomyces chromolaenae Mapook \& K.D. Hyde, Fungal Diversity 101: 118 (2020)

Dyfrolomyces distoseptatus M. Niranjan \& V.V. Sarma, Fungal Diversity 10.1007/s13225-020-00462-6: 57 (2020)

Dyfrolomyces neothailandicus Dayarathne, Jones E.B.G. \& K.D. Hyde, Mycosphere 11 (1): 78 (2020)

*Echinoderma flavidoasperum Y.J. Hou \& Z.W. Ge, Phytotaxa 447 (4): 223 (2020)

*Efibula yunnanensis C.L. Zhao, Phytotaxa 451: 242 (2020)

Elaphomyces bucholtzii Saitta, A. Paz, E. 
Otsing \& Tedersoo, Persoonia 44: 387 (2020)

Elaphomyces dalemurphyi Castellano \& R.A. Healy, Fungal Systematics and Evolution 7: 114 (2020)

Elaphomyces dunlapii Castellano, D. Mitchell \& Crabtree, Fungal Systematics and Evolution 7: 117 (2020)

Elaphomyces fuscus M. Shirakawa, Mycoscience 61 (6): 319 (2020)

Elaphomyces holstii Castellano \& R.A. Healy, Fungal Systematics and Evolution 7: 117 (2020)

Elaphomyces lougehrigii Castellano, R.A. Healy \& Marzitelli, Fungal Systematics and Evolution 7: 121 (2020)

Elaphomyces marmoratus M. Shirakawa, Mycoscience 61 (6): 316 (2020)

Elaphomyces miketroutii Castellano, Fungal Systematics and Evolution 7: 123 (2020)

Elaphomyces nemoreus Jeppson, Molia \& E. Larss., Persoonia 45: 347 (2020)

Elaphomyces roodyi Castellano \& D. Mitchell, Fungal Systematics and Evolution 7: 123 (2020)

Elaphomyces stevemilleri Castellano, Fungal Systematics and Evolution 7: 126 (2020)

Elaphomyces wazhazhensis Crabtree \& Castellano, Fungal Systematics and Evolution 7: 128 (2020)

Elongaticollum hedychii D.S. Tennakoon, C.H. Kuo \& K.D. Hyde, MycoKeys 70: 70 (2020)

*Elongaticonidia rosae W.J. Li \& Camporesi \& K.D. Hyde, Fungal Diversity 100: 516 (2020)

Elsinoe eucalyptorum Crous \& Summerell, Fungal Systematics and Evolution 6: 186 (2020)

Endococcus epiacarosporus Cl. Roux, Catalogue des lichens et champignons lichénicoles de France métropolitaine. 3e édition revue et augmentée 1: 1279 (2020)

Endoconidioma euphorbiae Crous, Persoonia
45: 301 (2020)

Endogone botryocarpa Koh. Yamam., Degawa \& A. Yamada, Mycologia 112 (2): 316 (2020)

Enterographa dokdoensis S.Y. Kondr., Lökös, B.G. Lee \& Hur, Acta bot. hung. 62 (3-4): 239 (2020)

Enterographa kinabaluensis Sparrius \& Kalb, Plant and Fungal Systematics 65 (1): 185 (2020)

Enterographa serusiauxii Lebreton \& Aptroot, Plant and Fungal Systematics 65 (1): 131 (2020)

Entoloma atricolor O.V. Morozova, Noordel., E.S. Popov \& A.V. Alexandrova, Index Fungorum 459: 1 (2020)

Entoloma canadense Noordel., G.M. Jansen \& Dima, Sydowia 72: 274 (2020)

Entoloma frigidum Noordel., E. Larss., Bendiksen, G.M. Jansen \& Dima, Sydowia 72: 277 (2020)

*Entoloma lushanense J. Wang, J.Q. Yan \& G.H. Huo, Phytotaxa 464 (1): 97 (2020)

Entoloma porphyroleucum O.V. Morozova, Noordel. \& Dima, Sydowia 72: 278 (2020)

Entoloma sequestratum T.F. Elliott, S.L. Stephenson, Karun. \& D. Nelsen, Fungal Systematics and Evolution 6: 254 (2020)

Entoloma vilaboense Blanco-Dios, Yesca 32: 70 (2020)

*Epichloe sinensis P. Tian, C.J. Li \& Z.B. Nan, Mycol. Progr. 19 (10): 1072 (2020)

*Epicoccum brahmanense L.W. Hou, L. Cai \& Crous, Stud. Mycol. 96: 340 (2020)

*Epicoccum dickmanii L.W. Hou \& O. Yarden, Stud. Mycol. 96: 341 (2020)

*Epicoccum longiostiolatum L.W. Hou, L. Cai \& Crous, Stud. Mycol. 96: 343 (2020)

*Epicoccum multiceps L.W. Hou, L. Cai \& Crous, Stud. Mycol. 96: 344 (2020)

*Epicoccum polychromum L.W. Hou, L. Cai \& Crous, Stud. Mycol. 96: 346 (2020)

*Epicoccum variabile L.W. Hou, L. Cai \& 
Crous, Stud. Mycol. 96: 349 (2020)

Epigloea diversispora McCune, Bryologist 123 (3): 535 (2020)

Eriocamporesia aurantia R.H. Perera, Samarak. \& K.D. Hyde, Fungal Diversity 100: 165 (2020)

Eriomyces heveae Huanraluek, Thambugala \& K.D. Hyde, Fungal Diversity 100: 147 (2020) Ernakulamia tanakae Rajeshkumar \& K.D. Hyde, Fungal Diversity 100: 111 (2020)

Erysiphe lonicerina S. Takam., Fungal Systematics and Evolution 7: 58 (2020)

Erysiphe medicaginis L. Kiss, L. Kelly \& Vaghefi, Persoonia 44: 389 (2020)

Erysiphe parvifoliae R. Kirschner, Phytotaxa 447 (4): 277 (2020)

Erysiphe pseudoviburni Y.J. Choi, H.D. Shin \& S. Takam., Mycologia 112 (3): 624 (2020)

Erysiphe viburniphila M. Bradshaw, Mycologia 112 (3): 621 (2020)

Erythrophylloporus flammans O.V. Morozova, T.H.G. Pham \& E.S. Popov, Sydowia 72: 281 (2020)

Eucasphaeria proteae Crous, Persoonia 45: 311 (2020)

*Exidia reflexa F. Wu, L.F. Fan \& S.Y. Ye, Mycol. Progr. 19 (9): 862 (2020)

*Exidia subglandulosa F. Wu, L.F. Fan \& S.Y. Ye, Mycol. Progr. 19 (9): 862 (2020)

*Exidia yadongensis $\mathrm{F}$. Wu, Qi Zhao, Zhu L. Yang \& Y.C. Dai, Mycosystema 39 (7): 8 (2020)

*Exophiala cinerea W. Sun, M.C. Xiang \& Xing Z. Liu, Journal of Fungi 6 (4, no. 187): 28 (2020)

*Exophiala clavispora W. Sun, M.C. Xiang \& Xing Z. Liu, Journal of Fungi 6 (4, no. 187): 29 (2020)

*Exophiala ellipsoidea W. Sun, L. Su, M.C. Xiang \& X.Z. Liu, Journal of Fungi 6 (4, no. 187): 29 (2020)

Exophiala embothrii Sand.-Den. \& A. Giraldo, Persoonia 45: 349 (2020)
Exophiala frigidotolerans Rodr.-Andr., Cano \& Stchigel, Persoonia 44: 391 (2020)

*Exophiala macquariensis C.D. Zhang \& Ferrari, Index Fungorum 461: 1 (2020)

Exophiala mali Crous, Persoonia 45: 285 (2020)

*Exophiala nagquensis W. Sun, L. Su, M.C. Xiang \& X.Z. Liu, Journal of Fungi 6 (4, no. 187): 30 (2020)

Exophiala prostantherae Crous, Persoonia 45: 291 (2020)

Exophiala quercina Crous \& R.K. Schumach., Fungal Systematics and Evolution 6: 187 (2020)

Exserticlava aquatica L.T. Carmo, C.R. Silva, Careli, S.M. Leão, Feletti \& Gusmão, Mycotaxon 134 (4): 732 (2020)

Falciformispora uttaraditensis Boonmee \& Huanraluek, Fungal Diversity 100: 127 (2020)

Farysia magdalenana Albu, Rush, Kijporn. \& Aime, Mycol. Progr. 19 (9): 929 (2020)

*Fasciatispora cocoes S.N. Zhang, K.D. Hyde \& J.K. Liu, Fungal Diversity 100: 231 (2020)

Fasciodontia brasiliensis Yurchenko \& Riebesehl, Mycol. Progr. 19 (2): 178 (2020)

*Filobasidium dingjieense Q.M. Wang, F.Y. Bai \& A.H. Li, Stud. Mycol. 96: 100 (2020) *Filobasidium globosum Q.M. Wang, F.Y. Bai \& A.H. Li, Stud. Mycol. 96: 100 (2020)

*Filobasidium mali Q.M. Wang, F.Y. Bai \& A.H. Li, Stud. Mycol. 96: 100 (2020)

*Filobasidium mucilaginum Q.M. Wang, F.Y. Bai \& A.H. Li, Stud. Mycol. 96: 103 (2020)

*Filsoniana lhasanensis X.M. Wen, Shahidin \& A. Abbas, Mycotaxon 134 (4): 669 (2020)

Fissurina excavatisorediosa Aptroot, Archive For Lichenology 16: 5 (2020)

Fissuroma arengae Konta \& K.D. Hyde, Acta Bot. Brasilica 34 (2): 388 (2020)

*Fissuroma palmae S.N. Zhang, K.D. Hyde \& J.K. Liu, Mycosphere 11 (1): 279 (2020)

Fissuroma wallichiae Konta \& K.D. Hyde, 
Acta Bot. Brasilica 34 (2): 388 (2020)

*Flavocillium bifurcatum H. Yu, Y.B. Wang, Y. Wang, Q. Fan \& Zhu L. Yang, Fungal Diversity 103: 20 (2020)

Floricola clematidis Phukhams., Camporesi \& K.D. Hyde, Fungal Diversity 10.1007/s13225-020-00448-4: 122 (2020)

Foliophoma camporesii D. Pem \& K.D. Hyde, Fungal Diversity 100: 28 (2020)

Follmannia suborthoclada S.Y. Kondr. \& Hur, Acta bot. hung. 62 (3-4): 243 (2020)

Fomitiporia bambusipileata Alves-Silva, Drechsler-Santos \& R.M.B. Silveira, Mycologia 112 (3): 637 (2020)

Fomitiporia conyana Alves-Silva \& Drechsler-Santos, Mycol. Progr. 19 (8): 781 (2020)

Fomitiporia ignea A.A. Brown, D.P. Lawr. \& K. Baumgartner, Pl. Path. 69 (2): 212 (2020)

Fomitiporia murrillii Alves-Silva, R.M. Silveira \& Drechsler-Santos, Mycol. Progr. 19 (8): 783 (2020)

Fomitiporia nubicola Alves-Silva, Bittencourt \& Drechsler-Santos, Mycol. Progr. 19 (8): 782 (2020)

Fomitiporia tsitsikamensis Tchotet, M.P.A. Coetzee, Rajchenb. \& Jol. Roux, Mycologia 112 (4): 728 (2020)

*Foraminispora yinggelingensis Y.F. Sun \& B.K. Cui, Persoonia 44: 220 (2020)

*Fraxinicola europaea Crous, M. Shen \& Y. Zhang ter, Stud. Mycol. 96: 217 (2020)

*Fraxinicola italica Crous, M. Shen \& Y. Zhang ter, Stud. Mycol. 96: 221 (2020)

Fulvifomes elaeodendri Tchotet, M.P.A. Coetzee, Rajchenb. \& Jol. Roux, Mycologia 112 (4): 732 (2020)

Fulvophyton serusiauxii Sparrius \& Tehler, Plant and Fungal Systematics 65 (1): 186 (2020)

Funneliformis pilosus A. Guillén, F.J. Serrano-Tamay, J.B. Peris \& I. Arrillaga, Mycologia 112 (4): 825 (2020)
Fusarium californicum D.P. Lawr., A. Stack, T.R. Gordon \& R.M. Bostock, Fungal Systematics and Evolution 6: 188 (2020)

Fusarium cassiae R.H. Perera, E.B.G. Jones \& K.D. Hyde, Mycosphere 11 (1): 2138 (2020)

Fusarium madaense C.N. Ezekiel, Sand.-Den., Houbraken \& Crous, MycoKeys 67: 112 (2020)

Fusarium magnoliae-champaca R.H. Perera, E.B.G. Jones \& K.D. Hyde, Mycosphere 11 (1): 2140 (2020)

Fusarium massalimae A.D. Cavalcanti, A.C.S. Santos, J.D.P. Bezerra \& O.M.C. Magalhães, Mycol. Progr. 19 (10): 1137 (2020)

Fusarium rekanum Lynn \& Marinc., Antonie van Leeuwenhoek 10.1007/s10482-02001392-8: 14 (2020)

Fusarium spartum S. Gargouri, V. Balmas, I. Laraba \& O'Donnell, Mycologia 112 (4): 799 (2020)

*Fusarium xiangyunense F. Zhang, X.Y. Yang \& K.D. Hyde, Phytotaxa 450 (3): 278 (2020)

Fusarium xyrophilum I. Laraba, K.J. Wurdack, Aime \& O'Donnell, Mycologia 112 (1): 45 (2020)

Fuscidea muskeg Tønsb. \& M. Zahradn., Lichenologist 52 (2): 94 (2020)

Fuscopannaria dillmaniae T. Sprib., Lichenologist 52 (2): 96 (2020)

*Fuscoporia australasiatica Q. Chen, F. Wu \& Y.C. Dai, Mycosphere 11 (1): 1482 (2020)

*Fuscoporia australiana Q. Chen, F. Wu \& Y.C. Dai, Mycosphere 11 (1): 1485 (2020)

*Fuscoporia bambusae Q. Chen, F. Wu \& Y.C. Dai, Mycosphere 11 (1): 1488 (2020)

*Fuscoporia chinensis Q. Chen, F. Wu \& Y.C. Dai, Mycosphere 11 (1): 1491 (2020)

*Fuscoporia eucalypti Q. Chen, F. Wu \& Y.C. Dai, Mycosphere 11 (1): 1493 (2020)

*Fuscoporia karsteniana Q. Chen, F. Wu \& Y.C. Dai, Mycosphere 11 (1): 1495 (2020)

Fuscoporia marquesiana Gibertoni \& C.R.S. 
de Lira, Fungal Diversity 10.1007/s13225020-00461-7: 129 (2020)

*Fuscoporia plumeriae Q. Chen, F. Wu \& Y.C. Dai, Mycosphere 11 (1): 1496 (2020)

Fuscoporia pulviniformis Tchotet, M.P.A. Coetzee, Rajchenb. \& Jol. Roux, Mycologia 112 (4): 734 (2020)

Fuscoporia semiarida Lima-Júnior, C.R.S. de Lira \& Gibertoni, Fungal Diversity 10.1007/s13225-020-00461-7: 132 (2020)

*Fuscoporia shoreae Q. Chen, F. Wu \& Y.C. Dai, Mycosphere 11 (1): 1497 (2020)

*Fuscoporia subchrysea Q. Chen, F. Wu \& Y.C. Dai, Mycosphere 11 (1): 1501 (2020)

Fuscostagonospora camporesii D.S Tennakoon, K.D Hyde, Fungal Diversity 100: 60 (2020)

Fusicolla cassiae-fistulae R.H. Perera, E.B.G. Jones \& K.D. Hyde, Mycosphere 11 (1): 2146 (2020)

Fusicolla gigantispora Dayarathne \& K.D. Hyde, Mycosphere 11 (1): 105 (2020)

Fusicolla siamensis R.H. Perera, E.B.G. Jones \& K.D. Hyde, Mycosphere 11 (1): 2148 (2020)

*Fusiformiseptata crocea W. Dong, H. Zhang \& K.D. Hyde, Fungal Diversity 10.1007/s13225-020-00463-5: 195 (2020)

Fusiformispora clematidis Phukhams. \& K.D. Hyde, Fungal Diversity 10.1007/s13225020-00448-4: 12 (2020)

*Gamszarea humicola Z.F. Zhang \& L. Cai, Fungal Diversity 10.1007/s13225-02000453-7: 73 (2020)

*Gamszarea lunata Z.F. Zhang \& L. Cai, Fungal Diversity 10.1007/s13225-02000453-7: 74 (2020)

*Gamszarea microspora Z.F. Zhang \& L. Cai, Fungal Diversity 10.1007/s13225-02000453-7: 75 (2020)

Ganoderma cinnamomea Ryvarden, Syn. Fung. 40: 103 (2020)

Ganoderma gabonensis Decock \& Ryvarden,
Syn. Fung. 42: 6 (2020)

Gassicurtia azorica Van den Boom, Acta bot. hung. 62 (3-4): 420 (2020)

Gassicurtia restingiana D.S. Andrade, M. Cáceres \& Aptroot, Bryologist 123 (1): 77 (2020)

Gastrosporium gossypinum T. Kasuya, S. Hanawa \& K. Hosaka, Truffology 2 (1): 11 (2020)

Geastrum calycicoriaceum Freitas-Neto, J.O. Sousa, Ovrebo, M.P. Martín \& Baseia, Persoonia 44: 393 (2020)

Genea coronata P. Alvarado, J.-B. Pérez \& M. Scholler, Sydowia 73: 2 (2020)

*Genolevuria bromeliarum Landell \& P. Valente ex Xin Zhan Liu, F.Y. Bai, M. Groenew. \& Boekhout, Stud. Mycol. 96: 133 (2020)

*Genolevuria pseudoamylolytica Q.M. Wang, F.Y. Bai \& A.H. Li, Stud. Mycol. 96: 83 (2020)

Geoglossum jirinae V. Kučera, Ševčíková \& Slovák, Persoonia 45: 351 (2020)

*Geomyces obovatus Zhi Y. Zhang, Y.F. Han \& Z.Q. Liang, Frontiers in Microbiology 11 (no. 572596): 5 (2020)

Ghazallomyces constrictus Hanafy, Lanjekar, Dhakephalkar, T.M. Callaghan, Dagar, G.W Griff., Elshahed \& N.H. Youssef, Mycologia 112 (6): 1233 (2020)

Gibellula cebrennini Tasan., Kuephadungphan \& Luangsa-ard, MycoKeys 72: 21 (2020)

Gibellula fusiformispora Tasan., Kuephadungphan \& Luangsa-ard, MycoKeys 72: 26 (2020)

Gibellula pigmentosinum Tasan., Kuephadungphan \& Luangsa-ard, MycoKeys 72: 27 (2020)

Gibellula scorpioides Tasan., Khons., Kuephadungphan \& Luangsa-ard, MycoKeys 72: 30 (2020)

Glaciozyma martinii Turchetti, Connell, 
Thomas-Hall \& Boekhout, Stud. Mycol. 96: 134 (2020)

Glaciozyma watsonii Thomas-Hall, Connell, Boekhout \& Turchetti, Stud. Mycol. 96: 134 (2020)

$\begin{array}{lcr}\text { Gliocephalotrichum } & \text { abrachium } & \text { R.A. } \\ \text { Fernandes, C.P. } & \text { Almeida \& } & \text { Pinho, } \\ \text { Mycologia 112(5): } 1010(2020) & \\ \text { Gliocephalotrichum } & \text { brasiliense } & \text { R.A. }\end{array}$ Fernandes, C.P. Almeida \& Pinho, Mycologia 112 (5): 1011 (2020)

Gliocephalotrichum caryocaris R.A. Fernandes, C.P. Almeida \& Pinho, Mycologia 112 (5): 1011 (2020)

Gliocladiopsis swieteniae R.H. Perera, E.B.G. Jones \& K.D. Hyde, Mycosphere 11 (1): 2153 (2020)

Globosporidium paramecii Y. Yakovleva, E. Nassonova, N. Lebedeva, O. Lanzoni, G. Petroni, A. Potekhin, E. Sabaneyeva, Parasitology 147 (9): 957-971 (2020)

Gloeandromyces hilleri Haelew. \& Pfliegler, Mycologia 112 (6): 1196 (2020)

*Gloeodontia yunnanensis C.L. Zhao, Phytotaxa 432 (2): 115 (2020)

Gloiothele olearia K.H. Larss., Melo \& Salcedo, Syn. Fung. 41: 13 (2020)

Glomus ibericum A. Guillén, F.J. SerranoTamay, J.B. Peris \& I. Arrillaga, Mycologia 112 (4): 822 (2020)

Glotzia balkanensis L.G. Valle \& D. Stoianova, MycoKeys 67: 59 (2020)

*Glugea eda L. Mansour, J.Y. Zhang, H.M. Abdel-Haleem, A.B. Darwish, S. AlQuraishy \& A.-A.S. Abdel-Baki, Acta Tropica 204 (no. 105331): 1 (2020)

Glyphis frischiana Kalb, Archive For Lichenology 15: 5 (2020)

*Gnomoniopsis chinensis C.M. Tian \& N. Jiang, MycoKeys 67: 24 (2020)

*Gnomoniopsis xunwuensis C.M. Tian \& Q. Yang, MycoKeys 69: 82 (2020)

Gobabebomyces vachelliae Crous, Persoonia
45: 273 (2020)

Gomphidius pseudoglutinosus K. Das, Hembrom, A. Parihar \& Vizzini, Cryptog. Mycol. 41 (4): 78 (2020)

Gongronella eborensis M.R. Martins, C. Santos, C. Soares, Cl. Santos \& N. Lima, Int. J. Syst. Evol. Microbiol. 70 (5): 3480 (2020) Gongronella hydei Doilom, Frontiers in Microbiology 11 (no. 585215): 7 (2020)

Gongronella namwonensis Hyang B. Lee, A.L. Santiago \& H.J. Lim, Persoonia 44: 395 (2020)

Gongronella pedratalhadensis L.W.S. Freitas, H.B. Lee \& A.L. Santiago, Sydowia 73: 65 (2020)

Gowardia zebrina T. Goward \& L. Myllys, Plant and Fungal Systematics 65: 223 (2020)

Graminopassalora geissorhizae Crous, Persoonia 45: 275 (2020)

Graphilbum acuminatum R. Jankowiak \& H. Solheim, Mycologia 112 (6): 1250 (2020)

Graphilbum carpaticum R. Jankowiak \& H. Solheim, Mycologia 112 (6): 1252 (2020)

Graphilbum curvidentis R. Jankowiak \& H. Solheim, Mycologia 112 (6): 1253 (2020)

Graphilbum furuicola R. Jankowiak \& H. Solheim, Mycologia 112 (6): 1254 (2020)

Graphilbum gorcense Marinc. \& R. Jankowiak, Mycologia 112 (6): 1257 (2020)

Graphilbum interstitiale R. Jankowiak \& H. Solheim, Mycologia 112 (6): 1255 (2020)

Graphilbum roseum Musvuugwa, L.L. Dreyer \& F. Roets, Mycol. Progr. 19 (1): 89 (2020)

Graphilbum sexdentatum R. Jankowiak \& H. Solheim, Mycologia 112 (6): 1256 (2020)

Graphis norsorediata Aptroot, Archive For Lichenology 16: 6 (2020)

Graphis tricolor Aptroot \& Feuerstein, Archive For Lichenology 16: 7 (2020)

Greeneria kielmeyerae C.P. Nicolli, F.S. Carmo, Inácio, P.A.S. Marbach \& J.T. De Souza, Persoonia 44: 397 (2020)

Grigorovia jiainica C.F. Lee \& Chun H. Liu ex 
Gouliamova \& Dimitrov, Comptes Rendus de l'Académie Bulgare des Sciences 73 (1): 55 (2020)

*Grosmannia tibetensis Z. Wang \& Q. Lu, Mycoscience 61 (6): 286 (2020)

*Grosmannia zekuensis R.L. Chang \& Z.W. de Beer, IMA Fungus 11 (no. 15): 11 (2020)

Gyalecta perithecioidea Aptroot, Archive For Lichenology 17: 6 (2020)

Gyalecta uniseptata Aptroot, Archive For Lichenology 17: 7 (2020)

Gyalidea nambourensis P.M. McCarthy, Australas. Lichenol. 87: 31 (2020)

Gymnascella minnisii J.D. Adam, A.E. ReaIreland, Smyth \& Overton, Persoonia 45: 353 (2020)

*Gymnoascus flavus Z.F. Zhang \& L. Cai, Fungal Diversity 10.1007/s13225-02000453-7: 54 (2020)

Gymnographopsis corticicola R. Miranda, Herrera-Camp. \& Lücking, Bryologist 123 (2): 132 (2020)

Gymnopilus dunensis H. Bashir, Jabeen \& Khalid, Phytotaxa 428 (1): 52 (2020)

Gymnopilus orientispectabilis Nagas., Malloch \& Thorn, Botany 98 (6): 304 (2020) Gymnopilus speciosissimus Y. Lamoureux, Malloch \& Thorn, Botany 98 (6): 307 (2020) Gymnopilus voitkii Malloch \& Thorn, Botany 98 (6): 295 (2020)

Gymnopus brunneiniger César, Bandala \& Montoya, Mycol. Progr. 19 (10): 1026 (2020)

Gymnopus brunneodiscus Antonín, Ryoo \& Ka, Mycobiology 48 (4): 257 (2020)

Gymnopus dryophiloides Antonín, Ryoo \& Ka, Mycobiology 48 (4): 255 (2020)

*Gymnosporangium kanas P. Zhao \& L. Cai, Persoonia 45: 97 (2020)

*Gymnosporangium lachrymiforme $\mathrm{P}$. Zhao \& L. Cai, Persoonia 45: 87 (2020)

*Gymnosporangium lianhuaense Y.M. Liang, B. Cao \& S.Q. Tao, Mycologia 112 (5): 956 (2020)
*Gymnosporangium shennongjiaense P. Zhao \& L. Cai, Persoonia 45: 93 (2020)

*Gymnosporangium spinulosum $\mathrm{P}$. Zhao \& L.

Cai, Persoonia 45: 93 (2020)

*Gymnosporangium tiankengense P. Zhao \&

L. Cai, Persoonia 45: 94 (2020)

* Gyromitra venenata Hai J. Li, Z.H. Chen \& Zhu L. Yang, Mycosystema 39 (9): 1712 (2020)

Gyroporus borealis Davoodian, O. Asher, Sturgeon, Ammirati \& Delaney, Phytotaxa 434: 214 (2020)

Gyroporus madagascariensis Buyck, O. Asher \& Davoodian, Phytotaxa 434: 212 (2020)

Gyroporus smithii Davoodian, Phytotaxa 434: 216 (2020)

Gyrothrix encephalarti Crous, Persoonia 44: 343 (2020)

*Haematomma pluriseptatum R. Tang, Mycotaxon 134 (4): 638 (2020)

*Haematomma rubidum R. Tang \& Z.T. Zhao, Mycotaxon 135 (2): 426 (2020)

Hafellia nortetrapla Aptroot, Oliveira Junior \& M. Cáceres, Bryologist 123 (4): 619 (2020)

Halecania athallina Fryday, Lichenologist 52 (2): 98 (2020)

Halocryptovalsa salicorniae Dayar. \& K.D. Hyde, Cryptog. Mycol. 41 (3): 52 (2020)

Halorosellinia krabiensis Dayarathne \& K.D. Hyde, Mycosphere 11 (1): 158 (2020)

Halorosellinia xylocarpi Dayarathne \& K.D. Hyde, Mycosphere 11 (1): 158 (2020)

Halotestudina muriformis Dayarathne \& K.D. Hyde, Mycosphere 11 (1): 69 (2020)

Hamatocanthoscypha podocarpi Crous, Persoonia 44: 327 (2020)

Hansenopezia decora Matočec, I. Kušan \& Jadan, Fungal Diversity 10.1007/s13225020-00461-7: 65 (2020)

Hantamomyces aloidendri Crous, Persoonia 45: 297 (2020)

Hebeloma adherens Monedero \& P. Alvarado, Yesca: 60 (2020) 
Hebeloma parvisporum Sparre Pedersen, Læssøe, Beker \& U. Eberh., Mycologia 112 (1): 179 (2020)

Hebeloma sagarae T. Kasuya, Mikami, Beker \& U. Eberh., Phytotaxa 456 (2): 134 (2020)

Heimioporus subcostatus Vadthanarat, Raspé \& Lumyong, Phytotaxa 475 (1): 21 (2020)

Heiomasia annamariae Kalb, Archive For Lichenology 15: 7 (2020)

Heiomasia siamensis Kalb, Archive For Lichenology 15: 8 (2020)

*Heitmania cylindrica Q.M. Wang, F.Y. Bai \& A.H. Li, Stud. Mycol. 96: 125 (2020)

*Heitmania tridendata Q.M. Wang, F.Y. Bai \& A.H. Li, Stud. Mycol. 96: 124 (2020)

*Helicoarctatus thailandicus D.F. Bao, Z.L. Luo, K.D. Hyde \& H.Y. Su, Fungal Diversity 10.1007/s13225-020-00461-7: 40 (2020)

Helicoascotaiwania lacustris Réblová \& J. Fourn., Stud. Mycol. 95: 453 (2020)

*Helicosporium thailandense $\mathrm{H}$. Zhang, W. Dong \& K.D. Hyde, Fungal Diversity 10.1007/s13225-020-00463-5: 206 (2020)

Heliocephala variabilis Iturrieta-González, Gené \& Dania García, Microorganisms 8 (4, no. 48): 7 (2020)

Helvella fuscolacunosa Skrede \& $\mathrm{T}$. Schumach., Fungal Systematics and Evolution 6: 80 (2020)

Helvella hispanica Skrede \& T. Schumach., Fungal Systematics and Evolution 6: 81 (2020)

Helvella iberica Skrede \& T. Schumach., Fungal Systematics and Evolution 6: 82 (2020)

Helvella inexpectata Skrede \& T. Schumach., Fungal Systematics and Evolution 6: 82 (2020)

Helvella neopallescens Skrede \& $\mathrm{T}$. Schumach., Fungal Systematics and Evolution 6: 85 (2020)

Helvella phlebophoroides Skrede \& $\mathrm{T}$.
Schumach., Fungal Systematics and Evolution 6: 86 (2020)

Helvella poculiformis Skrede \& T. Schumach., Fungal Systematics and Evolution 6: 88 (2020)

Helvella retinervis Skrede \& T. Schumach., Fungal Systematics and Evolution 6: 90 (2020)

*Helvella subtinta M. Zeng, Q. Zhao \& K.D. Hyde, Fungal Diversity 100: 160 (2020)

Helvella terricola Skrede \& T. Schumach., Fungal Systematics and Evolution 6: 91 (2020)

Hemileucoglossum kelabitense V. Kučera, Fedosova \& Sochorová, Persoonia 44: 399 (2020)

Hemitrichia crassifila A. Ronikier \& Lado, Mycologia 112 (4): 763 (2020)

Hermatomyces clematidis Phukhams., D.J. Bhat \& K.D. Hyde, Fungal Diversity 10.1007/s13225-020-00448-4: 34 (2020)

Herpomyces spegazzinii A.C. Gut. \& D. Haelew., Mycologia 112 (6): 1186 (2020)

Hesperomyces halyziae Haelew. \& De Kesel, MycoKeys 71: 31 (2020)

Heterocephalacria septentrionalis Kachalkin, Tomashevskaya \& Pankratov, Persoonia 44: 401 (2020)

*Heteroradulum yunnanense C.L. Zhao, Phytotaxa 437 (2): 57 (2020)

Heterosporicola beijingensis Brahmanage \& K.D. Hyde, Phytopath. Mediterr. 59 (2): 221 (2020)

*Hirsutella hongheensis D.P. Wei \& K.D. Hyde, Fungal Diversity 10.1007/s13225020-00461-7: 87 (2020)

Hirticrusta amazonica Matozaki, T. Hatt. \& Sotome, Mycoscience 61 (5): 245 (2020)

*Holtermannia saccardoi Q.M. Wang, F.Y. Bai \& A.H. Li, Stud. Mycol. 96: 99 (2020)

*Hongkongmyces aquaticus W. Dong, H. Zhang \& K.D. Hyde, Fungal Diversity 10.1007/s13225-020-00463-5: 105 (2020) 
*Hortiboletus arduinus N.K. Zeng, H.J. Xie \& W.F. Lin, Mycol. Progr. 19 (11): 1381 (2020)

*Hortiboletus napaeus N.K. Zeng, H.J. Xie, S. Jiang \& Zhi Q. Liang, Mycol. Progr. 19 (11): $1382(2020)$

Humidicutis brunneovinacea R. GaribayOrijel, Fungal Diversity 10.1007/s13225020-00461-7: 116 (2020)

*Huntiella hellenica F.F. Liu, Marinc. \& M.J. Wingf., MycoKeys 69: 38 (2020)

*Huntiella krugeri F.F. Liu, Marinc. \& M.J. Wingf., MycoKeys 69: 45 (2020)

Huriella falkusii Wilk, Lichenologist 52 (1): 39-45 (2020)

Huriella flakusii Wilk, Lichenologist 52 (1): 39 (2020)

Huriella upretiana S.Y. Kondr., G.K. Mishra, Nayaka \& A. Thell, Acta bot. hung. 62 (3-4): 351 (2020)

*Hyalinostachys cylindrospora C.G. Lin \& K.D. Hyde, Mycosphere 11 (1): 878 (2020)

Hyalorbilia caucasica Baral \& E. Weber, Monogr. Orbiliomycetes: 327 (2020)

*Hyalorbilia fagi E. Weber, Baral \& J.W. Guo, Monogr. Orbiliomycetes: 368 (2020)

Hyalorbilia fomentarii Baral, Monogr. Orbiliomycetes: 392 (2020)

Hyalorbilia helicospora Baral \& G. Marson, Monogr. Orbiliomycetes: 372 (2020)

Hyalorbilia herbicola Baral, Priou \& Perz, Monogr. Orbiliomycetes: 438 (2020)

Hyalorbilia hergiswiliana Baral, Polhorský, D. Savić \& E. Weber, Monogr. Orbiliomycetes: 433 (2020)

*Hyalorbilia japonica Baral, M.L. Wu \& Y.C. Su, Monogr. Orbiliomycetes: 354 (2020)

Hyalorbilia latispora Baral, G. Marson \& P. Perz, Monogr. Orbiliomycetes: 431 (2020)

*Hyalorbilia macrohelicospora Baral \& Hong Y. Su, Monogr. Orbiliomycetes: 376 (2020)

Hyalorbilia multiguttulata Baral \& B. Declercq, Monogr. Orbiliomycetes: 414 (2020)
Hyalorbilia orbiliicola Baral, G. Marson, Polhorský \& E. Weber, Monogr. Orbiliomycetes: 424 (2020)

Hyalorbilia pleioerythrostigma Baral, Monogr. Orbiliomycetes: 430 (2020)

Hyalorbilia puertoricensis Baral, Monogr. Orbiliomycetes: 334 (2020)

Hyalorbilia resinae Baral, Monogr. Orbiliomycetes: 437 (2020)

Hyalorbilia rotifera Baral \& E. Batten, Monogr. Orbiliomycetes: 440 (2020)

Hyalorbilia sibirica E. Weber \& Baral, Monogr. Orbiliomycetes: 375 (2020)

Hyalorbilia subfusispora Baral, G. Marson \& P. Perz, Monogr. Orbiliomycetes: 401 (2020) Hyalorbilia texensis Baral \& G. Marson, Monogr. Orbiliomycetes: 333 (2020)

*Hyalorbilia tortuosa Baral, E. Weber, Y.C. Su \& M.L. Wu, Monogr. Orbiliomycetes: 364 (2020)

Hyalorbilia ulicicola Baral \& Priou, Monogr. Orbiliomycetes: 322 (2020)

Hyalorbilia ulmi Baral \& E. Batten, Monogr. Orbiliomycetes: 436 (2020)

Hydrophilomyces deflexus Santam., Nova Hedwigia 110 (3-4): 350 (2020)

Hydrophilomyces riberae Santam., Nova Hedwigia 110 (3-4): 352 (2020)

Hydropisphaera angelicae Lechat, J. Fourn. \& Rubio, Ascomycete.org 12 (4): 175 (2020)

Hydropisphaera cirsii Lechat \& J. Fourn., Ascomycete.org 12 (2): 39 (2020)

Hydropisphaera pseudoarenula Lechat \& J. Fourn., Ascomycete.org 12 (2): 40 (2020)

Hydropisphaera saulensis Lechat \& J. Fourn., Ascomycete.org 12 (2): 45 (2020)

Hydropunctaria alaskana Thüs \& Pérez-Ort., Lichenologist 52 (2): 99 (2020)

Hygroaster andasibensis Ralaiv., Niskanen \& Liimat., Mycol. Progr. 19: 1295 (2020)

Hygroaster madagascarensis Ralaiv., Niskanen \& Liimat., Mycol. Progr. 19: 1297 (2020) 
*Hygrocybe debilipes C.Q. Wang \& T.H. Li, MycoKeys 75: 151 (2020)

*Hygrocybe griseonigricans C.Q. Wang \& T.H. Li, MycoKeys 75: 154 (2020)

*Hygrocybe rubroconica C.Q. Wang \& T.H. Li, MycoKeys 75: 156 (2020)

Hygrophorus betulae K. Bendiksen \& E. Larss., Karstenia 58: 4 (2020)

*Hygrophorus deliciosus C.Q. Wang \& T.H. Li, Phytotaxa 449 (3): 235 (2020)

Hygrophorus neoerubescens Papetti, Peintner \& Simonini, Riv. Micol. 62 (3): 201 (2020)

Hymenochaete boddingii Hembrom, A. Parihar, K. Das, A. Ghosh, Cryptog. Mycol. 41 (4): 83 (2020)

Hymenochaete longisterigmata Nav. Kaur, Avn. P. Singh \& Dhingra, Mycotaxon 135 (3): 632 (2020)

Hymenotorrendiella communis Crous \& P.R. Johnst., Persoonia 44: 349 (2020)

Hymenotorrendiella indonesiana Crous \& P.R. Johnst., Persoonia 44: 349 (2020)

Hyperphyscia lucida van der Pluijm, Lindbergia 43 (linbg.01138): 1 (2020)

*Hyphodermella aurantiaca C.L. Zhao, Ann. bot. fenn. 58: 61-68 (2020)

Hyphopichia lachancei Brysch-Herzb., M. Groenew., Dlauchy, M. Seidel \& G. Péter, Antonie van Leeuwenhoek 113 (6): 777 (2020)

Hypomontagnella spongiphila Kuhnert, Fungal Diversity 10.1007/s13225-02000447-5: 17 (2020)

Hypomyces gamsii Crous \& Akulov, Fungal Systematics and Evolution 6: 192 (2020)

*Hypomyces pseudolactifluorum F.M. Yu, Q. Zhao \& K.D. Hyde, Biodiversity Data Journal 8 (e53490): 11 (2020)

Hypotrachyna appalachensis Lendemer \& J.L. Allen, Bryologist 123 (2): 296 (2020)

*Hypotrachyna corallifera Xin Y. Wang \& Li S. Wang, Cryptog. Mycol. 41 (12): 198 (2020)
Hypotrachyna kauffmaniana Lendemer \& J.L. Allen, Bryologist 123 (2): 305 (2020)

Hypotrachyna longicilia, Cryptog. Mycol. 41 (12): 200 (2020)

Hypotrachyna mcmulliniana Lendemer \& J.L. Allen, Bryologist 123 (2): 307 (2020)

Hypotrachyna puerensis, Cryptog. Mycol. 41 (12): 202 (2020)

Hypotrachyna yunnana, Cryptog. Mycol. 41 (12): 205 (2020)

Hypoxylon aurantium Dayarathne, E.B.G. Jones \& K.D. Hyde, Mycosphere 11 (1): 151 (2020)

Hypoxylon baruense Cedeño-Sanchez, L. Wendt \& L.C. Mejía, Mycosphere 11 (1): 1464 (2020)

Hypoxylon bellicolor Cedeño-Sanchez, L. Wendt \& L.C. Mejía, Mycosphere 11 (1): 1464 (2020)

Hypoxylon delonicis R.H. Perera, E.B.G. Jones \& K.D. Hyde, Mycosphere 11 (1): 2169 (2020)

Hypoxylon guilanense Pourmogh. \& C. Lamb., MycoKeys 66: 112 (2020)

Hypoxylon hepaticolor J. Fourn. \& A.N. Mill., Persoonia 45: 357 (2020)

Hypoxylon mangrovei Dayarathne, E.B.G. Jones \& K.D. Hyde, Mycosphere 11 (1): 151 (2020)

Hypoxylon sporistriatatunicum CedeñoSanchez, L. Wendt \& L.C. Mejía, Mycosphere 11 (1): 1466 (2020)

*Hypoxylon wujiangense Y.H. Pi \& Q.R. Li, Phytotaxa 455 (1): 27 (2020)

Hysterangium bonobo M.E. Sm. \& T.F. Elliott, Mycologia 10.1080/00275514.2020.1790234: 5 (2020)

Ikaeria serusiauxii Sipman, Plant and Fungal Systematics 65 (1): 122 (2020)

*Ilyonectria changbaiensis X. Lu \& W. Gao, Journal of Ginseng Research 44: 513 (2020)

*Ilyonectria communis X. Lu \& W. Gao, Journal of Ginseng Research 44: 514 (2020) 
*Ilyonectria qitaiheensis X. Lu \& W. Gao, Journal of Ginseng Research 44: 514 (2020)

Ilyonectria vivaria Berlanas, LópezManzanares, Bujanda \& Gramaje, Pl. Dis. 104 (1): 98 (2020)

Imleria floridana A. Farid, A.R. Franck \& J. Bolin, Mycologia 112 (2): 425 (2020)

Induratia brasiliensis L.C. Pena, Servienski \& V. Kava ex Samarak., Thongbai, K.D. Hyde \& M. Stadler, Fungal Diversity 101: 193 (2020)

Induratia darjeelingensis Meshram, N. Kapoor \& S. Saxena ex Samarak., Thongbai, K.D. Hyde \& M. Stadler, Fungal Diversity 101: 193 (2020)

Induratia fengyangensis Chu L. Zhang ex Samarak., Thongbai, K.D. Hyde \& M. Stadler, Fungal Diversity 101: 193 (2020)

Induratia heveae Siri-Udom \& Lumyong ex Samarak., Thongbai, K.D. Hyde \& M. Stadler, Fungal Diversity 101: 193 (2020)

Induratia kashay Meshram, N. Kapoor \& S. Saxena ex Samarak., Thongbai, K.D. Hyde \& M. Stadler, Fungal Diversity 101: 193 (2020)

Induratia thailandica Samarak., Thongbai, K.D. Hyde \& M. Stadler, Fungal Diversity 101: 194 (2020)

Induratia tigerensis S. Saxena, Meshram \& N. Kapoor ex Samarak., Thongbai, K.D. Hyde \& M. Stadler, Fungal Diversity 101: 196 (2020)

Induratia yunnanensis C.L. Zhang ex Samarak., Thongbai, K.D. Hyde \& M. Stadler, Fungal Diversity 101: 196 (2020)

Induratia ziziphi Samarak., Thongbai, K.D. Hyde \& M. Stadler, Fungal Diversity 101: 196 (2020)

Infundibulicybe macrospora M. Ali, J. Khan, Niazi \& Khalid, Phytotaxa 452 (4): 271 (2020)

Inocephalus appressus Largent, Mycotaxon 135 (4): 792 (2020)
Inocybe amelandica Bandini \& B. Oertel, Mycol. bavarica 20: 23 (2020)

Inocybe antoniniana E. Sesli, Bandini \& Krisai, Sydowia 72: 98 (2020)

Inocybe astraiana Bandini \& B. Oertel, Mycol. bavarica 20: 27 (2020)

Inocybe athenana Bandini, B. Oertel \& U. Eberh., Mycol. bavarica 20: 33 (2020)

Inocybe castorina Bandini \& B. Oertel, Mycol. bavarica 20: 38 (2020)

Inocybe cavalcantiae Wartchow, Stud. Fung. 5 (1): 2 (2020)

Inocybe cuniculina Bandini \& B. Oertel, Mycol. bavarica 20: 42 (2020)

Inocybe digitula Bandini, Christan \& B. Oertel, Mycol. bavarica 20: 45 (2020)

*Inocybe gansuensis T. Bau \& Y.G. Fan, Mycosystema 39 (9): 1701 (2020)

Inocybe globulina Bandini \& B. Oertel, Mycol. bavarica 20: 49 (2020)

Inocybe ionolepis Cullington \& E. Larss., Persoonia 45: 359 (2020)

Inocybe laurina Bandini, B. Oertel \& C. Hahn, Mycol. bavarica 20: 65 (2020)

Inocybe lechiana Bandini, B. Oertel \& U. Eberh., Mycol. bavarica 20: 71 (2020)

Inocybe messapica Cervini, Phytotaxa 480 (2): 175 (2020)

Inocybe mimeana Bandini \& B. Oertel, Mycol. bavarica 20: 76 (2020)

*Inocybe muricellatoides T. Bau \& Y.G. Fan, Mycosystema 39 (9): 1700 (2020)

Inocybe perchtana Bandini \& B. Oertel, Mycol. bavarica 20: 80 (2020)

Inocybe pluppiana Bandini, B. Oertel \& U. Eberh., Mycol. bavarica 20: 86 (2020)

Inocybe vestalis Bandini, Weholt \& B. Oertel, Mycol. bavarica 20: 90 (2020)

Inocybe woglindeana Bandini, Vauras \& Weholt, Karstenia 58 (1): 47 (2020)

Inonotus novoguineensis Ryvarden, Syn. Fung. 40: 112 (2020)

Inonotus vitis A.A. Brown, D.P. Lawr. \& K. 
Baumgartner, Pl. Path. 69 (2): 213 (2020)

Ioplaca rinodinoides S.Y. Kondr., K.K. Ingle, D.K. Upreti \& S. Nayaka, Acta bot. hung. 62 (1-2): 77 (2020)

*Jattaea reniformis Z.F. Zhang \& L. Cai, Fungal Diversity 10.1007/s13225-02000453-7: 95 (2020)

Jimgerdemannia ambigua Koh. Yamam., Degawa \& A. Yamada, Mycologia 112 (2): 318 (2020)

*Jirovecia sinensis X.H. Liu, M.Q. Wen, Y.L. Zhao, A.H. Li \& J.Y. Zhang, J. Invert. Path. 173: 10.1016/j.jip.2020.107368: 4 (2020)

Joblinomyces apicalis Hanafy, Lanjekar, Dhakephalkar, T.M. Callaghan, Dagar, G.W. Griff., Elshahed \& N.H. Youssef, Mycologia 112 (6): 1233 (2020)

Juncomyces californiensis Crous, Persoonia 44: 333 (2020)

Juncomyces patwiniorum Crous, Persoonia 45: 277 (2020)

*Junghuhnia austrosinensis $\mathrm{F}$. Wu, P. Du \& X.M. Tian, MycoKeys 72: 5 (2020)

Junghuhnia mininitida Ryvarden, Syn. Fung. 40: 103 (2020)

*Junghuhnia nandinae F. Wu, P. Du \& X.M. Tian, MycoKeys 72: 8 (2020)

*Junghuhnia subcollabens F. Wu, P. Du \& X.M. Tian, MycoKeys 72: 10 (2020)

*Juxtiphoma kolkmaniarum Hern.-Restr., L.W. Hou, L. Cai \& Crous, MycoKeys 65: 76 (2020)

Kaarikia abrahamsonii C. Mayers, T.C. Harr. \& Roeper, Mycologia 112 (6): 1126 (2020)

Kalmusia araucariae Crous, Fungal Systematics and Evolution 6: 195 (2020)

Kalmusia erioi Samarak., Thambugala \& K.D. Hyde, Fungal Diversity 100: 47 (2020)

Kazachstania bozae Gouliamova \& Dimitrov, Index Fungorum 432: 1 (2020)

Kazachstania chrysolinae Gouliamova \& Dimitrov, Index Fungorum 432: 1 (2020)

Keissleriella camporesiana Phukhams.,
Camporesi. \& K.D. Hyde, Fungal Diversity 100: 63 (2020)

*Keissleriella camporesii C.G. Lin \& K.D. Hyde, Fungal Diversity 100: 67 (2020)

Keissleriella italica Brahmanage, Camporesi \& K.D. Hyde, Mycosphere 11 (1): 2490 (2020)

*Kernia anthracina Lei Su, H. Zhu \& C. Qin, Scientific Reports 10 (no. 10302): 7 (2020)

Khoyollomyces ramosus Hanafy, Lanjekar, Dhakephalkar, T.M. Callaghan, Dagar, G.W. Griff., Elshahed \& N.H. Youssef, Mycologia 112 (6): 1234 (2020)

Kiliophora clavatophora J.S. Monteiro, Cantillo \& Gusmão, Nova Hedwigia 110: 178 (2020)

*Knufia calcicola L. Su, W. Sun \& M.C. Xiang, Journal of Fungi 6 (4, no. 187): 18 (2020)

*Knufia separata L. Su, W. Sun \& M.C. Xiang, Journal of Fungi 6 (4, no. 187): 19 (2020)

*Kockovaella haikouensis Q.M. Wang, F.Y. Bai \& A.H. Li, Stud. Mycol. 96: 81 (2020)

*Kockovaella ischaemi Q.M. Wang, F.Y. Bai \& A.H. Li, Stud. Mycol. 96: 81 (2020)

*Kockovaella mexicana Lopandić, O. Molnár \& Prillinger ex ex Xin Zhan Liu, F.Y. Bai, M. Groenew. \& Boekhout, Stud. Mycol. 96: 134 (2020)

*Kockovaella nitrophila Q.M. Wang, F.Y. Bai \& A.H. Li, Stud. Mycol. 96: 83 (2020)

*Kondoa arboricola Q.M. Wang, F.Y. Bai \& A.H. Li, Stud. Mycol. 96: 104 (2020)

*Kondoa chamaenerii Q.M. Wang, F.Y. Bai \& A.H. Li, Stud. Mycol. 96: 104 (2020)

*Kondoa cylindrica Q.M. Wang, F.Y. Bai \& A.H. Li, Stud. Mycol. 96: 103 (2020)

*Kondoa daliangziensis Q.M. Wang, F.Y. Bai \& A.H. Li, Stud. Mycol. 96: 105 (2020)

*Kondoa foliicola Q.M. Wang, F.Y. Bai \& A.H. Li, Stud. Mycol. 96: 104 (2020)

*Kondoa lulangica Q.M. Wang, F.Y. Bai \& A.H. Li, Stud. Mycol. 96: 105 (2020)

*Kondoa myxariophila J.P. Samp., Q.M. 
Wang \& F.Y. Bai, Stud. Mycol. 96: 106 (2020)

*Kondoa rhododendri Q.M. Wang, F.Y. Bai \&

A.H. Li, Stud. Mycol. 96: 107 (2020)

*Kondoa ribitophobia Q.M. Wang, F.Y. Bai \&

A.H. Li, Stud. Mycol. 96: 105 (2020)

*Kondoa thailandica Fungsin, Hamam. \& Nakase ex Q.M. Wang, M. Groenew., F.Y. Bai \& Boekhout, Stud. Mycol. 96: 134 (2020)

Kosmimatomyces alatophylus Bianchin., Reinoso-Fuentealba, Rodr.-Andr., Cano \& Stchigel, Persoonia 44: 403 (2020)

Kukwaea pubescens Motiej. \& Zhurb., Phytotaxa 459 (1): 41 (2020)

Kwanghwana miscanthi Karun., C.H. Kuo \& K.D. Hyde, Cryptog. Mycol. 41 (6): 124 (2020)

*Kwoniella newhampshirensis K. Sylvester, Q.M. Wang \& Hittinger, Stud. Mycol. 96: 134 (2020)

*Kwoniella ovata Q.M. Wang, F.Y. Bai \& A.H. Li, Stud. Mycol. 96: 86 (2020)

*Kwoniella shandongensis R. Chen, Yuan M. Jiang \& S.C. Wei ex M. Groenew. \& Q.M. Wang, Stud. Mycol. 96: 134 (2020)

Laboulbenia assingii W. Rossi \& M. Leonardi, Cryptog. Mycol. 41 (1): 2 (2020)

Laboulbenia brachymetrae A. Weir, W. Rossi \& Kaishian, Mycologia 112 (3): 571 (2020)

Laboulbenia chionophila Santam., Nova Hedwigia 110 (3-4): 353 (2020)

Laboulbenia cylindrostethi A. Weir, W. Rossi \& Kaishian, Mycologia 112 (3): 572 (2020)

Laboulbenia halophila Santam. \& CuestaSegura, Nova Hedwigia 110 (3-4): 356 (2020)

Laboulbenia neogerris A. Weir, W. Rossi \& Kaishian, Mycologia 112 (3): 574 (2020)

Laboulbenia quarantenae Haelew. \& De Kesel, MycoKeys 71: 35 (2020)

Laboulbenia rugosa W. Rossi \& M. Leonardi, Cryptog. Mycol. 41 (1): 2 (2020)

Laboulbenia tachygerris A. Weir, W. Rossi \&
Kaishian, Mycologia 112 (3): 574 (2020)

Laccaria dallingii Corrales, Ovrebo, A.W. Wilson \& G.M. Mueller, Frontiers in Microbiology: 4 (2020)

*Laccaria fengkaiensis Fang Li, Mycol. Progr. 19 (5): 530 (2020)

Laccaria fortunensis Corrales, Ovrebo, A.W. Wilson \& G.M. Mueller, Frontiers in Microbiology 11 (no. 1597): 7 (2020)

Laccaria griseolilacina H.J. Cho \& Y.W. Lim, Mycobiology 48 (4): 291 (2020)

Laccaria macrobasidia H.J. Cho \& Y.W. Lim, Mycobiology 48 (4): 290 (2020)

Laccaria nitrophila Corrales, Ovrebo, A.W. Wilson \& G.M. Mueller, Frontiers in Microbiology 11 (no. 1597): 6 (2020)

*Laccaria prava Fang Li, Mycol. Progr. 19 (5): 533 (2020)

Lacrima galapagoensis Bungartz \& Søchting, Plant and Fungal Systematics 65 (2): 538 (2020)

Lacrymaria malayana S.M.L. Lee \& Voto, Rivista Micologica Romana, Boll. AMER 110 (2): 90 (2020)

Lacrymaria splendens S.M.L. Lee \& Voto, Rivista Micologica Romana, Boll. AMER 110 (2): 90 (2020)

Lacrymaria verrucispora S.M.L. Lee \& Voto, Rivista Micologica Romana, Boll. AMER 110 (2): 90 (2020)

Lactifluus albopicrus T. Lebel \& L. Tegart, Persoonia 44: 405 (2020)

Lactifluus austropiperatus T. Lebel \& L. Tegart, Persoonia 44: 407 (2020)

Lactifluus catarinensis J. Duque, M.A. Neves \& M. Jaegger, Syst. Biodiv. 18 (4): 353 (2020)

Lactifluus guadeloupensis Delgat \& Courtec., Persoonia 44: 288 (2020)

Lactifluus lactiglaucus P.L. Leonard \& J. Dearnaley, Persoonia 45: 361 (2020)

Lactifluus lepus Delgat \& Courtec., Persoonia 44: 292 (2020) 
Lactifluus marielleae J. Duque \& M.A. Neves, Syst. Biodiv. 18 (4): 354 (2020)

Lactifluus marmoratus Delgat, Persoonia 44: 288 (2020)

Lactifluus umbilicatus Silva-Filho, D.L. Komura \& Wartchow, Phytotaxa 436 (3): 224 (2020)

Lactifluus venosellus Silva-Filho, Sá \& Wartchow, Phytotaxa 436 (3): 227 (2020)

Lambiella aliphatica T. Sprib. \& Resl, Lichenologist 52 (2): 101 (2020)

Lanspora cylindrospora Devadatha, V.V. Sarma \& E.B.G. Jones, Fungal Diversity 100: $189(2020)$

Lasiodiplodia krabiensis Dayarathne, Mycosphere 11 (1): 75 (2020)

Lasiodiplodia mitidjana A. Alves, A.E. Mahamedi \& A. Berraf-Tebbal, PLoS ONE 15 (5): $\mathrm{e} 0232448,8$ (2020)

Lasioloma corticola P.M. McCarthy, Australas. Lichenol. 87: 58 (2020)

Lecania hydrophobica T. Sprib. \& Fryday, Lichenologist 52 (2): 103 (2020)

Lecanicillium gracile Ponizovskaya, A.A. Grum-Grzhim., Georgieva \& Bilanenko, Phytotaxa 443 (3): 268 (2020)

*Lecanicillium magnisporum Z.F. Zhang \& L. Cai, Fungal Diversity 10.1007/s13225-02000453-7: 75 (2020)

Lecanicillium praecognitum Gorczak \& Kisło, Persoonia 44: 409 (2020)

Lecanographa atlantica Ertz \& van den Boom, Phytotaxa 472 (2): 152 (2020)

Lecanora atro-ocellata Bungartz, Phytotaxa 431 (1): 26 (2020)

Lecanora baekdudaeganensis B.G. Lee \& Hur, MycoKeys 70: 46 (2020)

Lecanora cactacea Bungartz \& Elix, Phytotaxa 431 (1): 40 (2020)

Lecanora cerebriformis Bungartz \& Aptroot, Phytotaxa 431 (1): 23 (2020)

Lecanora cerebrosorediata Aptroot \& Bungartz, Phytotaxa 431 (1): 28 (2020)
Lecanora confusoides Bungartz \& Printzen, Phytotaxa 431 (1): 62 (2020)

Lecanora darwiniana Bungartz \& Elix, Phytotaxa 431 (1): 42 (2020)

Lecanora kalbii Bungartz \& Elix, Phytotaxa 431 (1): 48 (2020)

Lecanora malagae Bungartz \& Elix, Phytotaxa 431 (1): 52 (2020)

Lecanora muscigena Øvstedal \& Fryday, N.Z. Jl Bot. 58: 146 (2020)

Lecanora ombligulata Kalb, Bungartz \& Elix, Phytotaxa 431 (1): 53 (2020)

Lecanora pyrrhosporoides Bungartz, Elix \& Printzen, Phytotaxa 431 (1): 65 (2020)

Lecanora subaureoides Aptroot \& Bungartz, Phytotaxa 431 (1): 69 (2020)

Lecanora terpenoidea Bungartz \& Elix, Phytotaxa 431 (1): 71 (2020)

Lecanora viridipruinosa M. Svenss. \& T. Sprib., Lichenologist 52 (2): 105 (2020)

Lecidea buellielloides S.Y. Kondr. \& Hur, Acta bot. hung. 62 (3-4): 246 (2020)

Lecidea griseomarginata Fryday, Lichenologist 52 (2): 106 (2020)

Lecidea streveleri T. Sprib., Lichenologist 52 (2): 107 (2020)

Lecophagus ellipsoideus Baral \& G. Marson, Monogr. Orbiliomycetes: 291 (2020)

Lecophagus pini Baral, Monogr. Orbiliomycetes: 295 (2020)

Lecophagus subglobosus Baral, B. Coué \& M. Hairaud, Monogr. Orbiliomycetes: 296 (2020)

Lectera sambuci Crous \& Bulgakov, Fungal Systematics and Evolution 6: 196 (2020)

Legaliana subsaniosa Donadini \& Van Vooren, Cahiers de la FMBDS 7: 61 (2020)

Lentaria gossypina R. Salas-Lizana, M. Villegas \& E. Pérez-Pazos, Fungal Diversity 10.1007/s13225-020-00461-7: 124 (2020)

Lentaria variabilis M. Villegas, R. GaribayOrijel \& N. Matías-Ferrer, Fungal Diversity 10.1007/s13225-020-00461-7: 126 (2020) 
*Lentistoma aquaticum W. Dong, H. Zhang \& K.D. Hyde, Fungal Diversity 10.1007/s13225-020-00463-5: 127 (2020)

*Lentithecium kunmingense W. Dong, H. Zhang \& K.D. Hyde, Fungal Diversity 10.1007/s13225-020-00463-5: 96 (2020)

Lepiota cholistanensis H. Bashir, Usman \& Khalid, Phytotaxa 455 (4): 273 (2020)

*Lepiota omninoflava Y.J. Hou \& Z.W. Ge, Phytotaxa 447 (4): 226 (2020)

Lepiota punaensis Stallman, Mycotaxon 135 (3): 477 (2020)

Lepra arida A.W. Archer \& Elix, Australas. Lichenol. 86: 14 (2020)

Lepra elatinica A.W. Archer \& Elix, Australas. Lichenol. 86: 15 (2020)

Lepra perlacericans A.W. Archer \& Elix, Australas. Lichenol. 86: 16 (2020)

Leprocaulon beechingii Lendemer, Bryologist 123 (1): 6 (2020)

*Lepteutypa qujingensis Dissan., J.C. Kang \& K.D. Hyde, Phytotaxa 446 (3): 150 (2020)

Leptobacillium symbioticum Okane, Nonaka, Kurihara, J.P. Abe \& Yamaoka, Mycoscience 61 (4): 167 (2020)

Leptographium alni B. Strzałka, R. Jankowiak \& G. Hausner, MycoKeys 68: 39 (2020)

Leptographium doddsii Marinc., Z.W. de Beer \& M.J. Wingf., Persoonia 45: 185 (2020)

Leptographium gordonii Marinc., Z.W. de Beer \& M.J. Wingf., Persoonia 45: 185 (2020)

Leptographium owenii Marinc., Z.W. de Beer \& M.J. Wingf., Persoonia 45: 185 (2020)

Leptographium raffai Marinc., Z.W. de Beer \& M.J. Wingf., Persoonia 45: 188 (2020)

Leptographium seifertii Marinc., Z.W. de Beer \& M.J. Wingf., Persoonia 45: 187 (2020)

*Leptographium wushanense Y. Pan, J. Lu \& H. Ye, Mycoscience 61 (1): 45 (2020)

Leptosillia cordylines Senan. \& K.D. Hyde, Phytotaxa 435 (3): 217 (2020)

Leptosphaeria regiae D. Pem, Selcuk, Jeewon
\& K.D. Hyde, Frontiers in Microbiology 11 (no. 1303): 7 (2020)

*Leptosphaerulina obtusispora L.W. Hou, L. Cai \& Crous, Stud. Mycol. 96: 384 (2020)

*Leptosphaerulina sisyrinchiicola L.W. Hou,

L. Cai \& Crous, Stud. Mycol. 96: 385 (2020)

Leptospora chromolaenae Mapook \& K.D. Hyde, Fungal Diversity 101: 65 (2020)

Leptospora clematidis Phukhams., Ertz, Gerstmans \& K.D. Hyde, Fungal Diversity 10.1007/s13225-020-00448-4: 77 (2020)

Leptospora phraeana Mapook \& K.D. Hyde, Fungal Diversity 101: 65 (2020)

Leptosporella elaeidis Konta \& K.D. Hyde, Mycosphere 11 (1): 683 (2020)

Leptoxyphium citri Abdollahz. \& Crous, Stud. Mycol. 95: 402 (2020)

Letrouitia assamana S.Y. Kondr., G.K. Mishra \& D.K. Upreti, Acta bot. hung. 62 (12): 79 (2020)

Leucaenicola camelliae H.A. Ariyaw., I. Tsai \& Thambug., Scientific Reports 10 (no. 12762): 12 (2020)

Leucaenicola osmanthi Ariyaw., I. Tsai \& Thambugala, Phytotaxa 437 (1): 28 (2020)

Leucaenicola taiwanensis H.A. Ariyaw., I. Tsai \& Thambug., Scientific Reports 10 (no. 12762): 13 (2020)

Leucangium microspermum Koh. Yamam., Hir. Sasaki, Ohmae \& Orihara, Truffology 3 (1): 5 (2020)

Leucoagaricus brunneus Z. Ullah, Jabeen \& Khalid, Mycotaxon 134 (4): 603 (2020)

Leucoagaricus callainitinctus K.P.D. Latha, K.N.A. Raj \& Manim., Phytotaxa 442 (2): 113 (2020)

Leucoagaricus rhodelephantinus Boisselet \& Eyssart., Bull. Soc. mycol. Fr. 133 (3-4): 265 (2020)

Leucocoprinus domingensis Justo, Bizzi, Angelini \& Vizzini, Mycol. Progr. 19 (12): 1452 (2020)

Leucocoprinus parvipileus Justo, Bizzi, 
Angelini \& Vizzini, Mycol. Progr. 19 (12): 1449 (2020)

Leucocoprinus tephrolepis Justo, Bizzi, Angelini \& Vizzini, Mycol. Progr. 19 (12): $1451(2020)$

*Liangia sinensis H. Yu, Y.B. Wang, Y. Wang, Z.H. Chen \& Zhu L. Yang, Fungal Diversity 103: 22 (2020)

Lichenopeltella mizerniana G. Worobiec, Mycol. Progr. 19 (8): 800 (2020)

*Ligninsphaeriopsis thailandica Phukhams. \& J.K. Feng, Mycosphere 11 (1): 1866 (2020) Lignosphaeria diospyrosa Bhunjun, Phukhams. \& K.D.

Hyde, Fungal Diversity 10.1007/s13225-02000458-2: 9 (2020)

Lilapila jurana Stöckli, G. Marson, Baral, U. Graf, Gilgen, Wergen \& E. Weber, Monogr. Orbiliomycetes: 269 (2020)

Lilapila oculispora Baral \& G. Marson, Monogr. Orbiliomycetes: 264 (2020)

Lilapila oculisporella G. Marson, Baral \& E. Weber, Monogr. Orbiliomycetes: 267 (2020)

*Lindgomyces aquaticus W. Dong, H. Zhang \& K.D. Hyde, Fungal Diversity 10.1007/s13225-020-00463-5: 110 (2020)

Linocarpon calami Konta \& K.D. Hyde, Mycosphere 11 (1): 686 (2020)

Linteromyces quintiniae Crous, Persoonia 45: 287 (2020)

Lipomyces amatsuensis A. Yamaz., Lorliam \& H. Kawas., Int. J. Syst. Evol. Microbiol. 10.1099/ijsem.0.003924: 21 (2020)

Lipomyces chibensis A. Yamaz., Lorliam \& $\mathrm{H}$. Kawas., Int. J. Syst. Evol. Microbiol. 10.1099/ijsem.0.003924: 20 (2020)

Lipomyces haiminakanus A. Yamaz. \& H. Kawas., Int. J. Syst. Evol. Microbiol. 10.1099/ijsem.0.003924: 23 (2020)

Lipomyces iriomotensis A. Yamaz. \& $\mathrm{H}$. Kawas., Int. J. Syst. Evol. Microbiol. 10.1099/ijsem.0.003924: 22 (2020)
Lipomyces kamogawensis A. Yamaz., Lorliam \& H. Kawas., Int. J. Syst. Evol. Microbiol. 10.1099/ijsem.0.003924: 20 (2020)

Lipomyces kiyosumicus A. Yamaz., Lorliam \& H. Kawas., Int. J. Syst. Evol. Microbiol. 10.1099/ijsem.0.003924: 18 (2020)

Lipomyces komiensis A. Yamaz. \& H. Kawas., Int. J. Syst. Evol. Microbiol. 10.1099/ijsem.0.003924: 23 (2020)

Lipomyces melibiosiraffinosiphilus A. Yamaz., Lorliam \& H. Kawas., Int. J. Syst. Evol. Microbiol. 10.1099/ijsem.0.003924: 18 (2020)

Lipomyces nakamensis A. Yamaz. \& H. Kawas., Int. J. Syst. Evol. Microbiol. 10.1099/ijsem.0.003924: 24 (2020)

Lipomyces sakishimensis A. Yamaz. \& H. Kawas., Int. J. Syst. Evol. Microbiol. 10.1099/ijsem.0.003924: 24 (2020)

Lipomyces taketomicus A. Yamaz. \& $\mathrm{H}$. Kawas., Int. J. Syst. Evol. Microbiol. 10.1099/ijsem.0.003924: 21 (2020)

Lipomyces yaeyamensis A. Yamaz. \& $\mathrm{H}$. Kawas., Int. J. Syst. Evol. Microbiol. 10.1099/ijsem.0.003924: 22 (2020)

*Lithohypha catenulata L. Su, W. Sun \& M.C. Xiang, Journal of Fungi 6 (4, no. 187): 20 (2020)

Lobothallia gangwondoana S.Y. Kondr., J.J. Woo \& Hur, Acta bot. hung. 62 (1-2): 81 (2020)

Longiappendispora chromolaenae Mapook \& K.D. Hyde, Fungal Diversity 101: 141 (2020)

*Longipedicellata aquatica W. Dong, H. Zhang \& K.D. Hyde, Fungal Diversity 10.1007/s13225-020-00463-5: 117 (2020)

Longiseptatispora curvata Crous \& Bulgakov, Fungal Systematics and Evolution 6: 198 (2020)

Longispora clematidis Phukhams. \& K.D. Hyde, Fungal Diversity 10.1007/s13225020-00448-4: 81 (2020)

Longistriata flava Sulzbacher, Orihara, 
Grebenc, M.P. Martín \& Baseia, MycoKeys 62: 60 (2020)

Lonicericola fuyuanensis Yasanthika, L.S. Dissan., Monkai J, K.D. Hyde, Phytotaxa 446 (2): 109 (2020)

Lopharia erubescens V. Xavier de Lima, Mycol. Progr. 19: 34 (2020)

Lophotrichus medusoides Calvert, McTaggart \& R.G. Shivas, Persoonia 45: 363 (2020)

Loratospora arezzoensis Bundhun, Wanas., R. Jeewon \& K.D. Hyde, Fungal Diversity 100: 97 (2020)

*Luteoporia citriniporia Z.B. Liu \& Yuan Yuan, Phytotaxa 46 (1): 36 (2020)

Lycoperdon lahorense N. Yousaf \& A.N. Khalid, Fungal Diversity 10.1007/s13225020-00461-7: 101 (2020)

Lycoperdon pseudocurtisii N. Yousaf \& A.N. Khalid, Fungal Diversity 10.1007/s13225020-00461-7: 103 (2020)

*Lylea obclavata L. Qiu, Jian Ma, X.G. Zhang \& R.F. Castañeda, Mycotaxon 135 (4): 786 (2020)

*Lyomyces bambusinus C.L. Zhao, MycoKeys 65: 105 (2020)

*Lyomyces cremeus C.L. Zhao, MycoKeys 65: 108 (2020)

*Lyomyces macrosporus C.L. Zhao, MycoKeys 65: 108 (2020)

*Lyomyces wuliangshanensis C.L. Zhao, MycoKeys 65: 111 (2020)

Macalpinomyces collinsiae J. Kruse, M.N. Lyons, McTaggart \& R.G. Shivas, Persoonia 44: 411 (2020)

*Macroascochyta grandis L.W. Hou, L. Cai \& Crous, Stud. Mycol. 96: 333 (2020)

Macroconstrictolumina megalateralis Aptroot, Fungal Diversity 10.1007/s13225020-00462-6: 169 (2020)

Macrocybe sardoa Vizzini, Consiglio, M. Marchetti, Fungal Diversity 101: 247 (2020)

*Macroventuria angustispora L.W. Hou, L. Cai \& Crous, Stud. Mycol. 96: 362 (2020)
*Macroventuria terrestris L.W. Hou, L. Cai \& Crous, Stud. Mycol. 96: 364 (2020)

Magnaporthiopsis cynodontis P.L. Vines \& Tom.-Pet., Mycologia 112 (1): 60 (2020)

Magnopulchromyces scorpiophorus L.B. Conc., Gusmão \& R.F. Castañeda, Fungal Diversity 10.1007/s13225-020-00461-7: 24 (2020)

Mallocybe crassivelata Ferisin, Bizio, EsteveRav.,Vizzini \& Dovana, Persoonia 44: 413 (2020)

Marantokordyana boliviana M. Piepenbr., Maike Hartmann, T.A. Hofm. \& M. Lutz, Mycol. Progr. 19 (4): 360 (2020)

Marantokordyana oberwinkleriana M. Piepenbr., Maike Hartmann, T.A. Hofm. \& M. Lutz, Mycol. Progr. 19 (4): 358 (2020)

Marasmiellus boreoorientalis Kiyashko, Sydowia 72: 282 (2020)

Marasmiellus diaphanus César, Bandala \& Montoya, Mycol. Progr. 19 (10): 1022 (2020) Marasmiellus longistipes Muh. Ali, Niazi, Khalid, Sydowia 72: 287 (2020)

Marasmius alienigenus J.S. Oliveira, Pl. Syst. Evol. 306 (no. 31): 31 (2020)

Marasmius altoribeirensis J.S. Oliveira, Persoonia 44: 251 (2020)

Marasmius ambicellularis J.S. Oliveira, Persoonia 44: 259 (2020)

Marasmius avellaneus J.S. Oliveira, Pl. Syst. Evol. 306 (no. 31): 8 (2020)

Marasmius gracilis J.S. Oliveira, Pl. Syst. Evol. 306 (no. 31): 10 (2020)

Marasmius hobbitorum J.S. Oliveira, Persoonia 44: 265 (2020)

Marasmius longibasidiatus J.S. Oliveira, Pl. Syst. Evol. 306 (no. 31): 29 (2020)

Marasmius luteoolivaceus J.S. Oliveira, Persoonia 44: 254 (2020)

Marasmius neocrinis-equi R.A. Koch \& Aime, Mycologia 112 (6): 1098 (2020)

Marasmius neotropicalis J.S. Oliveira, Persoonia 44: 254 (2020) 
Marasmius nidus-avis R.A. Koch, N. Siegel \& Aime, Mycologia 112 (6): 1092 (2020)

Marasmius pallidibrunneus J.S. Oliveira, Persoonia 44: 245 (2020)

Marasmius pseudoniveoaffinis J.S. Oliveira, Persoonia 44: 250 (2020)

Marasmius rhabarbarinoides J.S. Oliveira, Persoonia 44: 267 (2020)

Marasmius subputtemansii J.S. Oliveira, Pl. Syst. Evol. 306 (no. 31): 38 (2020)

Marasmius subvigintifolius J.S. Oliveira, Pl. Syst. Evol. 306 (no. 31): 13 (2020)

Marasmius venatifolius J.S. Oliveira, Persoonia 44: 258 (2020)

Marasmius ypyrangensis J.S. Oliveira, Pl. Syst. Evol. 306 (no. 31): 41 (2020)

Mariannaea atlantica A.L. Alves, A.C.S. Santos \& P.V. Tiago, Fungal Diversity 100: 203 (2020)

Massariosphaeria clematidis Phukhams., Wanas. \& K.D. Hyde, Fungal Diversity 10.1007/s13225-020-00448-4: 15 (2020)

Massjukiella rusavskioides S.Y. Kondr. \& Hur, Acta bot. hung. 62 (3-4): 249 (2020)

Mazosia corticola Kantvilas, Plant and Fungal Systematics 65 (2): 261 (2020)

*Melampsora salicis-michelsonii C.M. Tian \& L.L. Wang, Phytotaxa 435 (4): 287 (2020)

Melanconis larissae Jaklitsch \& Voglmayr, MycoKeys 63: 86 (2020)

Melanconis pacifica Jaklitsch \& Voglmayr, MycoKeys 63: 103 (2020)

*Melanographium phoenicis S.N. Zhang, K.D. Hyde \& J.K. Liu, Fungal Diversity 100: 235 (2020)

Melanoleuca dominicana Angelini, Para \& Vizzini, Persoonia 45: 365 (2020)

*Melanops fagicola W.J. Li, Camporesi \& K.D. Hyde, Fungal Diversity 100: 577 (2020)

Meliola elaeocarpicola Lini K. Mathew, J. Threatened Taxa 12 (5): 15672 (2020)

*Melnikomyces longisporus T.P. Wei \& Y.L. Jiang, Phytotaxa 471 (1): 64 (2020)
Melnikomyces thailandicus Giraldo López, Fungal Systematics and Evolution 6: 17 (2020)

Melomastia clematidis Phukhams. \& K.D. Hyde, Fungal Diversity 10.1007/s13225020-00448-4: 139 (2020)

Melomastia fulvicomae Phukhams. \& K.D. Hyde, Fungal Diversity 10.1007/s13225020-00448-4: 141 (2020)

Memnoniella chromolaenae Mapook \& K.D. Hyde, Fungal Diversity 101: 135 (2020)

* Mendogia calami H.B. Jiang, Phookamsak \& K.D. Hyde, Mycol. Progr. 19: 47 (2020)

*Mendogia chiangraiensis H.B. Jiang, Phookamsak \& K.D. Hyde, Mycol. Progr. 19: 45 (2020)

*Mendogia yunnanensis H.B. Jiang, Phookamsak \& K.D. Hyde, Mycol. Progr. 19: 48 (2020)

*Meniscomyces layueensis Q.M. Wang, F.Y. Bai \& A.H. Li, Stud. Mycol. 96: 114 (2020) *Meruliopsis leptocystidiata C.C. Chen \& Sheng H. Wu, Mycologia 112 (1): 72 (2020)

*Meruliopsis parvispora C.C. Chen \& Sheng H. Wu, Mycologia 112 (1): 76 (2020)

*Mesocorynespora sinensis Jian Ma, X.G. Zhang \& R.F. Castañeda, Mycotaxon 135 (3): 618 (2020)

Metarhizium biotecense Luangsa-ard, Khons., Thanakitp. \& Samson, Stud. Mycol. 95: 204 (2020)

Metarhizium candelabrum Luangsa-ard, Mongkols., Thanakitp. \& Samson, Stud. Mycol. 95: 207 (2020)

Metarhizium cercopidarum Luangsa-ard, Mongkols., Thanakitp. \& Samson, Stud. Mycol. 95: 209 (2020)

Metarhizium cicadae Luangsa-ard, Tasan., Thanakitp., \& Samson, Stud. Mycol. 95: 212 (2020)

Metarhizium clavatum Luangsa-ard, Mongkols., Lamlertthon, Thanakitp. \& Samson, Stud. Mycol. 95: 212 (2020) 
Metarhizium culicidarum Luangsa-ard, Khons., Thanakitp. \& Samson, Stud. Mycol. 95: 214 (2020)

Metarhizium eburneum Luangsa-ard, Noisrip., Thanakitp. \& Samson, Stud. Mycol. 95: 214 (2020)

Metarhizium ellipsoideum Luangsa-ard, Khons., Thanakitp. \& Samson, Stud. Mycol. 95: 216 (2020)

Metarhizium flavum Luangsa-ard, Mongkols., Thanakitp. \& Samson, Stud. Mycol. 95: 217 (2020)

Metarhizium fusoideum Luangsa-ard, Mongkols., Thanakitp. \& Samson, Stud. Mycol. 95: 217 (2020)

Metarhizium gryllidicola Khons., Thanakitp. \& Luangsa-ard, Persoonia 44: 150 (2020)

Metarhizium huainamdangense Luangsa-ard, Mongkols., Thanakitp. \& Samson, Stud. Mycol. 95: 222 (2020)

Metarhizium megapomponiae Luangsa-ard, Tasan., Thanakitp. \& Samson, Stud. Mycol. 95: 226 (2020)

Metarhizium niveum Luangsa-ard, Tasan., Thanakitp. \& Samson, Stud. Mycol. 95: 226 (2020)

Metarhizium nornnoi Luangsa-ard, Khons., Thanakitp. \& Samson, Stud. Mycol. 95: 227 (2020)

Metarhizium ovoidosporum Luangsa-ard, Khons., Thanakitp. \& Samson, Stud. Mycol. 95: 227 (2020)

Metarhizium phasmatodeae Khons., Thanakitp. \& Luangsa-ard, Persoonia 44: 151 (2020)

Metarhizium phuwiangense Luangsa-ard, Mongkols., Himaman, Thanakitp. \& Samson, Stud. Mycol. 95: 232 (2020)

Metarhizium purpureonigrum Luangsa-ard, Tasan., Thanakitp. \& Samson, Stud. Mycol. 95: 233 (2020)

Metarhizium purpureum Luangsa-ard, Mongkols., Lamlertthon, Thanakitp. \&
Samson, Stud. Mycol. 95: 234 (2020)

Metarhizium sulphureum Luangsa-ard, Khons., Thanakitp. \& Samson, Stud. Mycol. 95: 237 (2020)

Metschnikowia amazonensis A.R.O. Santos, C.A. Rosa \& Lachance, Yeast 37 (3): 259 (2020)

Metschnikowia lacustris Lachance, Antonie van Leeuwenhoek 113 (6): 760 (2020)

Micarea crassa P.M. McCarthy \& Elix, Australas. Lichenol. 87: 63 (2020)

Micarea minuta van den Boom, GuzowKrzem. \& Kukwa, Plant and Fungal Systematics 65 (1): 194 (2020)

Micarea pseudotsugae van den Boom, GuzowKrzem. \& Kukwa, Plant and Fungal Systematics 65 (1): 197 (2020)

Micarea queenslandica P.M. McCarthy \& Elix, Australas. Lichenol. 87: 65 (2020)

Micarea rubiformis P.M. McCarthy \& Elix, Australas. Lichenol. 87: 26 (2020)

*Microascus collaris Z.F. Zhang \& L. Cai, Fungal Diversity 10.1007/s13225-02000453-7: 83 (2020)

*Microascus levis Z.F. Zhang \& L. Cai, Fungal Diversity 10.1007/s13225-020-00453-7: 83 (2020)

*Microascus sparsimycelialis Z.F. Zhang \& L. Cai, Fungal Diversity 10.1007/s13225-02000453-7: 85 (2020)

*Microascus superficialis Z.F. Zhang \& L. Cai, Fungal Diversity 10.1007/s13225-02000453-7: 86 (2020)

*Microascus trigonus Z.F. Zhang \& L. Cai, Fungal Diversity 10.1007/s13225-02000453-7: 86 (2020)

*Microbotryozyma swertiae Q.M. Wang, F.Y. Bai \& A.H. Li, Stud. Mycol. 96: 125 (2020)

*Microdochium dawsoniorum C. Lock, Vitelli, Holdom, Y.P. Tan \& R.G. Shivas, Persoonia 44: 417 (2020)

*Microdochium indocalami S.T. Huang, J.W. Xia, X.G. Zhang, W.X. Sun \& Z. Li, 
MycoKeys 72: 100 (2020)

*Microdochium yunnanense S.T. Huang, J.W. Xia, X.G. Zhang, W.X. Sun \& Z. Li, MycoKeys 72: 101 (2020)

Microporus nigroglaber Decock \& Ryvarden, Syn. Fung. 42: 9 (2020)

*Microsphaeropsis fusca L.W. Hou, L. Cai \& Crous, Stud. Mycol. 96: 371 (2020)

*Microsphaeropsis taxicola L.W. Hou, L. Cai \& Crous, Stud. Mycol. 96: 373 (2020)

*Microsphaeropsis viridis L.W. Hou, L. Cai \& Crous, Stud. Mycol. 96: 374 (2020)

*Microsporomyces ellipsoideus Q.M. Wang, F.Y. Bai \& A.H. Li, Stud. Mycol. 96: 114 (2020)

*Microsporomyces pseudomagnisporus Q.M. Wang, F.Y. Bai \& A.H. Li, Stud. Mycol. 96: 115 (2020)

*Microsporomyces rubellus Q.M. Wang, F.Y. Bai \& A.H. Li, Stud. Mycol. 96: 115 (2020) Milesina thailandica Y. Ono, Unartngam \& Okane, Mycol. Progr. 19 (2): 151 (2020)

Minutiella pruni-avium S. Bien \& Damm, MycoKeys 63: 144 (2020)

Minutiella simplex C. Kraus, Damm, S. Bien, Vögele \& M. Fisch., Fungal Systematics and Evolution 6: 145 (2020)

Miriquidica gyrizans Fryday, Lichenologist 52 (2): 110 (2020)

Modicella albostipitata J.A. Cooper, Phytotaxa 453 (3): 294 (2020)

*Moelleriella gracilispora Jun Z. Qiu \& Y.X. Chen, Fungal Diversity 10.1007/s13225020-00461-7: 78 (2020)

Moelleriella puertoricoensis Mongkols., Noisrip. \& Luangsa-ard, Persoonia 45: 367 (2020)

*Moelleriella sinensis Jun Z. Qiu \& Y.X. Chen, Phytotaxa 429 (4): 292 (2020)

Mollisia diesbachiana Tanney \& Seifert, Stud. Mycol. 95: 331 (2020)

Mollisia gibbospora I. Kušan, Matočec, Pošta, Tkalčec \& Mešić, Persoonia 44: 419 (2020)
Mollisia monilioides Tanney \& Seifert, Stud. Mycol. 95: 331 (2020)

Mollisia novobrunsvicensis Tanney \& Seifert, Stud. Mycol. 95: 335 (2020)

Mollisia prismatica Tanney \& Seifert, Stud. Mycol. 95: 336 (2020)

Mollisia rava Tanney \& Seifert, Stud. Mycol. 95: 341 (2020)

Moniliophthora mayarum Lodge, Aime \& Niveiro, MycoKeys 66: 43 (2020)

Monoicomyces drusillae Santam., Nova Hedwigia 110 (3-4): 359 (2020)

Montagnula camporesii Phukhams., Camporesi. \& K.D. Hyde, Fungal Diversity 100: 51 (2020)

Montagnula chiangraiensis Mapook \& K.D. Hyde, Fungal Diversity 101: 29 (2020)

Montagnula chromolaenae Mapook \& K.D. Hyde, Fungal Diversity 101: 34 (2020)

Montagnula chromolaenicola Mapook \& K.D. Hyde, Fungal Diversity 101: 35 (2020)

Montagnula cylindrospora Valenz.-Lopez, Cano, Guarro \& Stchigel, Persoonia 44: 421 (2020)

Montagnula thailandica Mapook \& K.D. Hyde, Fungal Diversity 101: 35 (2020)

Morchella iberica Marcos Martínez, Sanjaume \& Clowez, Ascomycete.org 12 (1): 11 (2020) Moringomyces phantasmae Crous, Persoonia 45: 323 (2020)

Moristroma germanicum C. Kraus, Damm, S. Bien, Vögele \& M. Fisch., Fungal Systematics and Evolution 6: 148 (2020)

Moristroma palatinum C. Kraus, Damm, S. Bien, Vögele \& M. Fisch., Fungal Systematics and Evolution 6: 148 (2020)

Mortierella multispora Tibpromma, Karun., Karasaki \& P.E. Mortimer, Emerging Microbes \& Infections 9 (1): 1558 (2020)

Mortierella rhinolophicola Tibpromma, Karun., Karasaki \& P.E. Mortimer, Emerging Microbes \& Infections 9 (1): 1559 (2020) 
Mortierella yunnanensis Tibpromma, Karun., Karasaki \& P.E. Mortimer, Emerging Microbes \& Infections 9 (1): 1561 (2020)

Mrakia montana $\mathrm{B}$. Turchetti \& P. Buzzini, Int. J. Syst. Evol. Microbiol. 70 (8): 4709 (2020)

*Mrakia panshiensis R.R. Jia \& F.L. Hui, MycoKeys 74: 82 (2020)

Mrakia stelviica B. Turchetti \& P. Buzzini, Int. J. Syst. Evol. Microbiol. 70 (8): 4707 (2020) *Mucispora infundibulata J. Yang \& K.D. Hyde, Mycosphere 11 (1): 591 (2020)

Multiclavula petricola $\mathrm{H}$. Masumoto \& Y. Degawa, Mycoscience 61 (4): 157 (2020)

\section{Murichromolaenicola chiangraiensis}

Mapook \& K.D. Hyde, Fungal Diversity 101: 72 (2020)

Murichromolaenicola chromolaenae Mapook \& K.D. Hyde, Fungal Diversity 101: 72 (2020)

Murinectria murispora M. Niranjan \& V.V. Sarma, Mycosphere 11 (1): 738 (2020)

Muriphila oklahomaensis Jurjević, Čmoková \& Hubka, Persoonia 44: 423 (2020)

Muyocopron chromolaenae Mapook \& K.D. Hyde, Fungal Diversity 101: 121 (2020)

Muyocopron chromolaenicola Mapook \& K.D. Hyde, Fungal Diversity 101: 123 (2020) Muyocopron thailandica S. Hongsanan, N. Huanraluek, K. Thambugala, Phytotaxa 456 (2): 198 (2020)

Mycena confinationis Ibarretxe, Fern.-Vic. \& Arnedo, Index Fungorum 463: 1 (2020)

*Mycena jingyinga C.C. Chang, C.Y. Chen, W.W. Lin \& H.W. Kao, Taiwania 65 (3): 399 (2020)

Mycena juniperifoliae Ibarretxe, Fern.-Vic. \& Arnedo, Index Fungorum 463: 1 (2020)

*Mycena luguensis C.C. Chang, C.Y. Chen, W.W. Lin \& H.W. Kao, Taiwania 65 (3): 401 (2020)

Mycena pradensis A. Tacconi, L. Setti, G. Maraia, Boll. Assoc. Micol. Ecol. Romana 109 (1): 4 (2020)
Mycena pulchra P.L. Leonard, Persoonia 45: 369 (2020)

*Mycena venus C.C. Chang, C.Y. Chen, W.W. Lin \& H.W. Kao, MycoKeys 65 (3): 403 (2020)

Mycoenterolobium aquadictyosporium M.S. Calabon, Boonmee, E.B.G. Jones \& K.D. Hyde, Mycol. Progr. 19 (10): 1035 (2020)

Mycorrhaphium ursinum Decock \& Ryvarden, Syn. Fung. 42: 26 (2020)

Mycosphaerangium quercinum Voglmayr, Jaklitsch \& Tello, Mycol. Progr. 19 (11): 1338 (2020)

Myriotrema robertianum Kalb, Archive For Lichenology 18: 4 (2020)

Myriotrema squamiferum Kalb, Archive For Lichenology 15: 11 (2020)

Myriotrema subzollingeri Kalb, Archive For Lichenology 18: 5 (2020)

Myrmecridium phragmiticola Crous \& Akulov, Persoonia 44: 329 (2020)

Naganishia onofrii Turchetti, Selbmann \& Zucconi ex Yurkov, Stud. Mycol. 96: 134 (2020)

Naganishia vaughanmartiniae Turchetti, Blanchette \& Arenz ex Yurkov, Stud. Mycol. 96: 134 (2020)

*Natonodosa speciosa Heredia, R.F. Castañeda \& D.W. Li, Mycol. Progr. 19 (1): 17 (2020)

Naviculispora terrestris Stchigel, Y. Marín, Cano \& Guarro, Microorganisms 8 (9, no. 1430): 15 (2020)

Navisporus laccatus C. Sharp \& Ryvarden, Syn. Fung. 40: 110 (2020)

Navisporus obscurus Ryvarden, Syn. Fung. 40: 104 (2020)

Nectria marina Dayarathne \& K.D. Hyde, Mycosphere 11 (1): 109 (2020)

Nemania phetchaburiensis Dayarathne, E.B.G. Jones \& K.D. Hyde, Mycosphere 11 (1): 165 (2020)

Nemania viridis Dayarathne \& K.D. Hyde, 
Mycosphere 11 (1): 162 (2020)

Neoantennariella phylicae Abdollahz. \& Crous, Stud. Mycol. 95: 406 (2020)

Neoasbolisia phylicae Abdollahz. \& Crous, Stud. Mycol. 95: 407 (2020)

*Neoascochyta dactylidis W.J. Li, Camporesi \& K.D. Hyde, Fungal Diversity 100: 604 (2020)

Neoascochyta fuci M. Gonçalves \& A. Alves, Int. J. Syst. Evol. Microbiol. 10.1099/ijsem.0.004410: 6 (2020)

*Neoascochyta fusiformis L.W. Hou, L. Cai \& Crous, Stud. Mycol. 96: 389 (2020)

*Neoascochyta humicola L.W. Hou, L. Cai \& Crous, Stud. Mycol. 96: 389 (2020)

*Neoascochyta longispora L.W. Hou, L. Cai \& Crous, Stud. Mycol. 96: 390 (2020)

*Neoascochyta mortariensis L.W. Hou, L. Cai \& Crous, Stud. Mycol. 96: 391 (2020)

*Neoastrosphaeriella phoenicis S.N. Zhang, E.B.G. Jones \& J.K. Liu, Mycosphere 11 (1): 279 (2020)

Neobrownliella salyangensis S.Y. Kondr. \& Hur, Acta bot. hung. 62 (3-4): 254 (2020)

Neobyssosphaeria clematidis Wanas., E.B.G. Jones \& K.D. Hyde, Fungal Diversity 10.1007/s13225-020-00448-4: 57 (2020)

Neocalonectria tristaniopsidis Crous, Persoonia 45: 271 (2020)

Neocamarosporium artemisiae Dayarathne \& E.B.G. Jones, Mycosphere 11 (1): 39 (2020) Neocamarosporium leipoldtiae Crous, Persoonia 45: 295 (2020)

Neocamarosporium maritimae Dayarathne \& E.B.G. Jones, Mycosphere 11 (1): 42 (2020) Neocladosporium osteospermi Crous, Persoonia 45: 309 (2020)

Neocladosporium syringae Crous \& Akulov, Fungal Systematics and Evolution 6: 203 (2020)

*Neocoleroa cameroonensis Crous, M. Shen \& Y. Zhang ter, Stud. Mycol. 96: 203 (2020) Neocosmospora magnoliae R.H. Perera,
E.B.G. Jones \& K.D. Hyde, Mycosphere 11 (1): 2158 (2020)

Neocosmospora pallidimors Tibpromma, Karun., Karasaki \& P.E. Mortimer, Emerging Microbes \& Infections 9 (1): 1562 (2020)

Neocosmospora rhizophorae Dayarathne, Mycosphere 11 (1): 112 (2020)

*Neocryphonectria carpini C.M. Tian, N. Jiang \& Crous, Mycologia 112 (2): 283 (2020)

*Neocryphonectria chinensis C.M. Tian, N. Jiang \& Crous, Mycologia 112 (2): 284 (2020)

*Neodactylaria simaoensis H. Zheng \& Z.F. Yu, MycoKeys 73: 76 (2020)

*Neodermea rossica W.J. Li, D.J. Bhat \& K.D. Hyde, Fungal Diversity 100: 613 (2020)

Neodevriesia aestuarina M. Gonçalves \& A. Alves, Persoonia 44: 425 (2020)

Neodevriesia manglicola Devadatha, V.V. Sarma \& E.B.G. Jones, Fungal Diversity 10.1007/s13225-020-00461-7: 16 (2020)

Neodidymelliopsis camporesii D. Pem \& K.D. Hyde, Fungal Diversity 100: 41 (2020)

Neodidymelliopsis salviae Brahamanage, Camporesi \& K.D. Hyde, Fungal Diversity 10.1007/s13225-020-00461-7: 20 (2020)

*Neodidymelliopsis tiliae L.W. Hou, L. Cai \& Crous, Stud. Mycol. 96: 387 (2020)

Neodidymelliopsis urticae Manawas., Camporesi \& K.D. Hyde, Fungal Diversity 10.1007/s13225-020-00461-7: 21 (2020)

Neodothiora populina Crous, G.C. Adams \& Winton, Persoonia 45: 325 (2020)

Neoerysiphe sechii Gregorio-Cipriano \& D. González, Botany 98 (4): 188 (2020)

Neofabraea eucalyptorum Crous, Persoonia 44: 337 (2020)

*Neofavolus americanus J.H. Xing, J.L. Zhou \& B.K. Cui, Mycol. Progr. 19 (5): 475 (2020)

*Neofavolus squamatus J.H. Xing, J.L. Zhou \& B.K. Cui, Mycol. Progr. 19 (5): 476 (2020) 
*Neofusicoccum dianense G.Q. Li \& S.F. Chen, IMA Fungus 11 (no. 22): 34 (2020)

*Neofusicoccum magniconidium G.Q. Li \& S.F. Chen, IMA Fungus 11 (no. 22): 36 (2020)

*Neofusicoccum ningerense G.Q. Li \& S.F. Chen, IMA Fungus 11 (no. 22): 37 (2020)

*Neofusicoccum parviconidium G.Q. Li \& S.F. Chen, IMA Fungus 11 (no. 22): 38 (2020)

*Neofusicoccum yunnanense G.Q. Li \& S.F. Chen, IMA Fungus 11 (no. 22): 39 (2020)

*Neogloeosporidina pruni W.J. Li, Camporesi \& K.D. Hyde, Fungal Diversity 100: 623 (2020)

*Neohelicascus submersus H. Yang, W. Dong, K.D. Hyde \& H. Zhang, Fungal Diversity 10.1007/s13225-020-00463-5: 150 (2020)

*Neohelicomyces dehongensis H. Zhang, W. Dong \& K.D. Hyde, Fungal Diversity 10.1007/s13225-020-00463-5: 212 (2020)

*Neohelicomyces thailandicus H. Zhang, W. Dong \& K.D. Hyde, Fungal Diversity 10.1007/s13225-020-00463-5: 212 (2020)

*Neohelicosporium submersum $\mathrm{H}$. Zhang, W. Dong \& K.D. Hyde, Fungal Diversity 10.1007/s13225-020-00463-5: 214 (2020)

*Neokalmusia kunmingensis H.B. Jiang, Phookamsak \& K.D. Hyde, Fungal Diversity 100: 51 (2020)

Neolamproconium silvestre Crous \& Akulov, Fungal Systematics and Evolution 6: 204 (2020)

Neoleptosporella clematidis Phukhams., Konta \& K.D. Hyde, Fungal Diversity 10.1007/s13225-020-00448-4: 151 (2020)

Neomassarina chromolaenae Mapook \& K.D. Hyde, Fungal Diversity 101: 52 (2020)

Neometulocladosporiella seifertii Crous, Persoonia 45: 303 (2020)

Neomicrosphaeropsis juglandis D. Pem, R. Jeewon, Selcuk \& K.D. Hyde, Frontiers in Microbiology 11 (no. 1303): 9 (2020)
*Neomonodictys muriformis Y.Z. Lu, C.G. Lin \& K.D. Hyde, Fungal Diversity 100: 207 (2020)

Neomyrmecridium asymmetricum R.F. Castañeda, Serrano \& D. Sosa, Mycotaxon 135 (1): 157 (2020)

*Neomyrmecridium guizhouense N.G. Liu, K.D. Hyde \& J.K. Liu, Fungal Diversity 100: 187 (2020)

Neoophiobolus chromolaenae Mapook \& K.D Hyde, Fungal Diversity 101: 75 (2020)

*Neopestalotiopsis acrostichi Norphanphoun, T.C. Wen \& K.D. Hyde, Mycosphere 10 (1): 540 (2020)

*Neopestalotiopsis brachiata Norphanphoun, T.C. Wen \& K.D. Hyde, Mycosphere 10 (1): 543 (2020)

*Neopestalotiopsis nebuloides C. Lock, Vitelli, Holdom, Y.P. Tan \& R.G. Shivas, Persoonia 44: 427 (2020)

*Neopestalotiopsis petila Norphanphoun, T.C. Wen \& K.D. Hyde, Mycosphere 10 (1): 543 (2020)

*Neopestalotiopsis rhizophorae Norphanphoun, T.C. Wen \& K.D. Hyde, Mycosphere 10 (1): 545 (2020)

*Neopestalotiopsis sonneratae Norphanphoun, T.C. Wen \& K.D. Hyde, Mycosphere 10 (1): 547 (2020)

Neopestalotiopsis thailandica Norphanphoun \& K.D. Hyde, Mycosphere 10 (1): 550 (2020) Neophaeomoniella constricta C. Kraus, Damm, S. Bien, Vögele \& M. Fisch., Fungal Systematics and Evolution 6: 149 (2020)

Neophaeomoniella ossiformis C. Kraus, Damm, S. Bien, Vögele \& M. Fisch., Fungal Systematics and Evolution 6: 151 (2020)

Neophysopella doipuiensis Okane \& Y. Ono, Mycol. Progr. 19 (9): 909 (2020)

Neophysopella sriphumensis Okane \& Y. Ono, Mycol. Progr. 19 (9): 913 (2020)

Neophysopella tropicalis Y. Ono, S. Chatasiri, Pota \& Okane, Mycol. Progr. 19 (9): 914 
(2020)

*Neopyrenochaeta annellidica W.J. Li, Z.H. Zhang \& K.D. Hyde, Fungal Diversity 100: 625 (2020)

*Neopyrenochaeta chiangraiensis Z.L. Luo, W.J. Li \& K.D. Hyde, Fungal Diversity 100: $626(2020)$

Neopyrenochaeta chromolaenae Mapook \& K.D. Hyde, Fungal Diversity 101: 60 (2020)

*Neopyrenochaeta maesuayensis Z.L. Luo, W.J. Li \& K.D. Hyde, Fungal Diversity 100: 628 (2020)

Neopyrenochaeta thailandica Mapook \& K.D. Hyde, Fungal Diversity 101: 62 (2020)

Neopyrenochaeta triseptatispora Mapook \& K.D. Hyde, Fungal Diversity 101: 63 (2020)

Neoroussoella clematidis Phukhams. \& K.D. Hyde, Fungal Diversity 10.1007/s13225020-00448-4: 105 (2020)

Neoroussoella fulvicomae Phukhams. \& K.D. Hyde, Fungal Diversity 10.1007/s13225020-00448-4: 105 (2020)

Neoroussoella lignicola A. Poli, E. Bovio, Prigione \& Varese, Diversity 12 (4, no. 144): 11 (2020)

Neoroussoella magnoliae N.I. de Silva \& K.D. Hyde, Fungal Diversity 10.1007/s13225020-00461-7: 32 (2020)

*Neosetophoma camporesii Q. Tian \& K.D. Hyde, Fungal Diversity 100: 99 (2020)

Neosetophoma hnaniceana Spetik, Eichmeier \& Berraf-Tebbal, Persoonia 44: 429 (2020)

Neoshiraia camelliae H.A. Ariyaw., I. Tsai \& Thambug., Scientific Reports 10 (no. 12762): 11 (2020)

Neoshiraia taiwanensis H.A. Ariyaw., I. Tsai \& Thambug., Scientific Reports 10 (no. 12762): 11 (2020)

Neosorocybe pini Crous \& Akulov, Fungal Systematics and Evolution 6: 207 (2020)

Neostictis nigricans Ekanayaka, Camporesi \& K.D. Hyde, Fungal Diversity 10.1007/s13225-020-00448-4: 143 (2020)
Neotorrubiella chinghridicola Tasan., Thanakitp. \& Luangsa-ard, Persoonia 44: 155 (2020)

*Neottiella gigaspora M. Zeng, Q. Zhao \& K.D. Hyde, Fungal Diversity 10.1007/s13225-020-00461-7: 70 (2020)

Neovaginatispora clematidis Phukhams. \& K.D. Hyde, Fungal Diversity 10.1007/s13225-020-00448-4: 45 (2020)

Neoxylaria arengae Konta \& K.D. Hyde, Mycosphere 11 (1): 2638 (2020)

Nephroma orvoi Timdal, M. Westb., Haugan, Hofton, Holien, Speed, Tønsberg \& Bendiksby, Graphis Scripta 32 (4): 78 (2020)

*Nielozyma formosana Nakase, Tsuzuki, F.L. Lee \& M. Takash. ex Xin Zhan Liu, F.Y. Bai, M. Groenew. \& Boekhout, Stud. Mycol. 96: 135 (2020)

*Nielozyma melastomatis Nakase, Tsuzuki, F.L. Lee \& M. Takash. ex Xin Zhan Liu, F.Y. Bai, M. Groenew. \& Boekhout, Stud. Mycol. 96: 135 (2020)

Niesslia peltigerae Pérez-Ort., Lichenologist 52 (2): 112 (2020)

*Nigrograna aquatica W. Dong, H. Zhang \& K.D. Hyde, Fungal Diversity 10.1007/s13225-020-00463-5: 74 (2020)

Nigrograna chromolaenae Mapook \& K.D. Hyde, Fungal Diversity 101: 50 (2020)

Nigrograna rhizophorae Dayarathne, E.B.G. Jones \& K.D. Hyde, Mycosphere 11 (1): 48 (2020)

Nigrograna samueliana Devadatha, V.V. Sarma \& E.B.G. Jones, Mycosphere 11 (1): 43 (2020)

*Nigrospora globosa Z.F. Zhang \& L. Cai, Fungal Diversity 10.1007/s13225-02000453-7: 97 (2020)

Nolanea albertinae Karstedt \& Capelari, Mycotaxon 135 (3): 596 (2020)

Nolanea atropapillata Karstedt \& Capelari, Mycotaxon 135 (3): 600 (2020)

Nolanea pallidosalmonea Karstedt \& Capelari, 
Mycotaxon 135 (3): 602 (2020)

Nolanea parvispora Karstedt \& Capelari, Mycotaxon 135 (3): 603 (2020)

Nolanea tricholomatoidea Karstedt \& Capelari, Mycotaxon 135 (3): 606 (2020)

*Nothophoma brennandiae Hern.-Restr., L.W. Hou, L. Cai \& Crous, MycoKeys 65: 77 (2020)

Nothophoma chromolaenae Mapook \& K.D. Hyde, Fungal Diversity 101: 18 (2020)

*Nothophoma infuscata L.W. Hou, L. Cai \& Crous, Stud. Mycol. 96: 368 (2020)

*Nothophoma nullicana L.W. Hou, L. Cai \& Crous, Stud. Mycol. 96: 369 (2020)

*Nothophoma spiraeae L.X. Zhang \& X.L. Fan, Phytotaxa 430 (3): 150 (2020)

Nothoseiridium podocarpi Crous, Persoonia 44: 323 (2020)

Nyungwea pyneei Ertz \& Diederich, Plant and Fungal Systematics 65 (1): 45 (2020)

*Oberwinklerozyma dicranopteridis Q.M. Wang, F.Y. Bai \& A.H. Li, Stud. Mycol. 96: 126 (2020)

*Oberwinklerozyma nepetae Q.M. Wang, F.Y. Bai \& A.H. Li, Stud. Mycol. 96: 126 (2020) *Oberwinklerozyma silvestris Scorzetti \& Golubev ex Q.M. Wang, F.Y. Bai, M. Groenew. \& Boekhout, Stud. Mycol. 96: 135 (2020)

*Oberwinklerozyma straminea Golubev \& Scorzetti ex Q.M. Wang, F.Y. Bai, M. Groenew. \& Boekhout, Stud. Mycol. 96: 135 (2020)

*Occultibambusa kunmingensis C.X. Liu, H. Zhang \& K.D. Hyde, Fungal Diversity 10.1007/s13225-020-00463-5: 153 (2020)

Oceanoplaca chemoisidiosa Søchting \& Bungartz, Plant and Fungal Systematics 65 (2): 540 (2020)

Oceanoplaca sideritoides Søchting \& Bungartz, Plant and Fungal Systematics 65 (2): 544 (2020)

Ocellularia jutaratiae Kalb, Archive For
Lichenology 18: 6 (2020)

Ocellularia macrocrocea Kalb, Archive For Lichenology 15: 14 (2020)

Ocellularia subnatashae Kalb, Archive For Lichenology 18: 7 (2020)

Ochraceocephala foeniculi Voglmayr \& Aiello, MycoKeys 66: 14 (2020)

Ochroconis ferulica Z. Tazik \& K. Rahnama, Nova Hedwigia 110 (3-4): 374 (2020)

*Ochroconis terricola Xin Zhang \& Y.L. Jiang, Mycotaxon 135 (1): 146 (2020)

Ochrolechia cooperi T. Sprib., Lichenologist 52 (2): 113 (2020)

Ochropsora staphyleae Y. Ono, S. Chatasir, E. Tanaka, Mycoscience 61 (2): 63 (2020)

Octospora doebbeleri Sochorová \& Eckstein, Sydowia 73: 239 (2020)

Okeanomyces marinus Calabon, E.B.G. Jones, Boonmee \& K.D. Hyde, Fungal Diversity 10.1007/s13225-020-00458-2: 28 (2020)

Ophiobolus lathyri Brahmanage, Camporesi \& K.D. Hyde, Mycosphere 11: 2515 (2020)

Ophiocordyceps campes Tasan., Thanakitp. \& Luangsa-ard, Mycol. Progr. 19 (10): 1045 (2020)

Ophiocordyceps corriemoreauae J.P.M. Araújo, H.C. Evans \& D.P. Hughes, Mycologia 112 (6): 1151 (2020)

Ophiocordyceps diabolica J.P.M. Araújo, H.C. Evans \& D.P. Hughes, Mycologia 112 (6): 1143 (2020)

Ophiocordyceps kobayasii Mongkols., Thanakitp., Luangsa-ard \& Hywel-Jones, Persoonia 44: 156 (2020)

Ophiocordyceps krachonicola Tasan., Thanakitp. \& Luangsa-ard, Persoonia 44: 158 (2020)

Ophiocordyceps longistromata Tasan., Thanakitp. \& Luangsa-ard, Mycol. Progr. 19 (10): 1047 (2020)

Ophiocordyceps odontomachi J.P.M. Araújo, H.C. Evans \& D.P. Hughes, Mycologia 112 (6): 1154 (2020) 
Ophiocordyceps paltothyrei J.P.M. Araújo, H.C. Evans \& D.P. Hughes, Mycologia 112 (6): 1143 (2020)

Ophiocordyceps phuwiangensis Tasan., Thanakitp., Phommavong \& Luangsa-ard, Mycol. Progr. 19 (10): 1052 (2020)

Ophiocordyceps pseudomyrmicis J.P.M. Araújo, H.C. Evans \& D.P. Hughes, Mycologia 112 (6): 1151 (2020)

Ophiosphaerella chiangraiensis Phookamsak \& K.D. Hyde, Fungal Diversity 10.1007/s13225-020-00461-7: 29 (2020)

Ophiosphaerella taiwanensis D.S. Tennakoon, C.H. Kuo \& K.D. Hyde, MycoKeys 70: 73 (2020)

*Ophiostoma genhense Z. Wang \& Q. Lu, IMA Fungus 11 (no. 3): 11 (2020)

Ophiostoma gilletteae Marinc., Z.W. de Beer \& M.J. Wingf., Persoonia 45: 189 (2020)

*Ophiostoma hongxingense Z. Wang \& Q. Lu, IMA Fungus 11 (no. 3): 15 (2020)

*Ophiostoma kunlunense R.L. Chang \& Z.W. de Beer, IMA Fungus 11 (no. 15): 10 (2020)

*Ophiostoma lotiforme Z. Wang \& Q. Lu, IMA Fungus 11 (no. 3): 17 (2020)

*Ophiostoma manchongi R.L. Chang \& Z.W. de Beer, IMA Fungus 11 (no. 15): 10 (2020)

*Ophiostoma multisynnematum Z. Wang \& Q. Lu, IMA Fungus 11 (no. 3): 18 (2020)

*Ophiostoma peniculi Z. Wang \& Q. Lu, IMA Fungus 11 (no. 3): 19 (2020)

*Ophiostoma pseudobicolor Z. Wang \& Q. Lu, IMA Fungus 11 (no. 3): 20 (2020)

Ophiostoma shanxiense Marinc., Z.W. de Beer \& M.J. Wingf., Persoonia 45: 189 (2020)

*Ophiostoma subelongati Z. Wang \& Q. Lu, IMA Fungus 11 (no. 3): 21 (2020)

*Ophiostoma xinganense Z. Wang \& Q. Lu, IMA Fungus 11 (no. 3): 22 (2020)

Orbilia acaciae Baral \& G. Marson, Monogr. Orbiliomycetes: 1072 (2020)

*Orbilia acicularis Baral \& Hong Y. Su,
Monogr. Orbiliomycetes: 1009 (2020)

Orbilia aethiopica Baral \& U. Lindem., Monogr. Orbiliomycetes: 912 (2020)

Orbilia albidorosea Baral \& G. Marson, Monogr. Orbiliomycetes: 939 (2020)

Orbilia albovinosa Baral, Monogr. Orbiliomycetes: 945 (2020)

Orbilia allantoobliqua Baral \& G. Marson, Monogr. Orbiliomycetes: 762 (2020)

Orbilia alpigena Baral \& E. Weber, Monogr. Orbiliomycetes: 595 (2020)

Orbilia amberina Baral \& G. Marson, Monogr. Orbiliomycetes: 1260 (2020)

Orbilia angiosubvinosa Baral, R. Tena \& E. Weber, Monogr. Orbiliomycetes: 620 (2020)

Orbilia anguliobliqua Baral \& G. Marson, Monogr. Orbiliomycetes: 758 (2020)

Orbilia angustoaristata Baral \& G. Marson, Monogr. Orbiliomycetes: 700 (2020)

Orbilia angustoobliqua Baral \& G. Marson, Monogr. Orbiliomycetes: 764 (2020)

Orbilia anigozanthi Baral \& G. Marson, Monogr. Orbiliomycetes: 1619 (2020)

Orbilia arachnopus Baral \& G. Marson, Monogr. Orbiliomycetes: 1305 (2020)

Orbilia arachnovinosa Baral \& E. Weber, Monogr. Orbiliomycetes: 622 (2020)

Orbilia aradi Baral \& G. Marson, Monogr. Orbiliomycetes: 777 (2020)

Orbilia arizonensis Baral \& G. Marson, Monogr. Orbiliomycetes: 553 (2020)

Orbilia astrovinosa Baral \& G. Marson, Monogr. Orbiliomycetes: 629 (2020)

Orbilia asturiensis Baral, E. Rubio \& J. Linde, Monogr. Orbiliomycetes: 686 (2020)

Orbilia atlantis Baral, Spooner \& Hairaud, Monogr. Orbiliomycetes: 1442 (2020)

Orbilia atriplicis Baral, Monogr. Orbiliomycetes: 1276 (2020)

Orbilia atrolentiformis Baral \& G. Marson, Monogr. Orbiliomycetes: 464 (2020)

Orbilia aureocrenulata Baral, Monogr. Orbiliomycetes: 1416 (2020) 
Orbilia australiensis Baral \& G. Marson, Monogr. Orbiliomycetes: 556 (2020)

Orbilia austrocylindrica Baral \& E. Weber, Monogr. Orbiliomycetes: 1317 (2020)

Orbilia austroobtusispora Baral \& G. Marson, Monogr. Orbiliomycetes: 1065 (2020)

Orbilia austroocculta Baral \& G. Marson, Monogr. Orbiliomycetes: 733 (2020)

Orbilia austropleiomicrosoma Baral \& G. Marson, Monogr. Orbiliomycetes: 1304 (2020)

Orbilia austroregalis Baral, Monogr. Orbiliomycetes: 776 (2020)

Orbilia aviaristata Baral \& G. Marson, Monogr. Orbiliomycetes: 740 (2020)

Orbilia aviceps Baral \& G. Marson, Monogr. Orbiliomycetes: 742 (2020)

Orbilia aviflagellata Baral \& G. Marson, Monogr. Orbiliomycetes: 738 (2020)

Orbilia bambusina Baral, Monogr. Orbiliomycetes: 1656 (2020)

Orbilia barrowensis Baral \& G. Marson, Monogr. Orbiliomycetes: 1199 (2020)

Orbilia basiflexa Baral, Monogr. Orbiliomycetes: 1279 (2020)

Orbilia battenii Baral \& E. Weber, Monogr. Orbiliomycetes: 1333 (2020)

Orbilia beatricis Baral, Monogr. Orbiliomycetes: 1322 (2020)

Orbilia bicknellensis Baral \& G. Marson, Monogr. Orbiliomycetes: 1129 (2020)

*Orbilia binchuanensis Baral \& Hong Y. Su, Monogr. Orbiliomycetes: 844 (2020)

Orbilia brachychitonis Baral \& G. Marson, Monogr. Orbiliomycetes: 784 (2020)

Orbilia brettii O. Ceska, Baral, G. Marson \& E. Weber, Monogr. Orbiliomycetes: 1323 (2020)

Orbilia breviaristata Baral, Priou \& G. Marson, Monogr. Orbiliomycetes: 724 (2020)

Orbilia breviclava Baral, Monogr. Orbiliomycetes: 689 (2020)

Orbilia cactacearum Baral \& G. Marson,
Monogr. Orbiliomycetes: 1143 (2020)

Orbilia calyptrata Baral \& G. Marson, Monogr. Orbiliomycetes: 1187 (2020)

Orbilia canadensis Baral \& G. Marson, Monogr. Orbiliomycetes: 1251 (2020)

Orbilia carminorosea Baral, Monogr. Orbiliomycetes: 906 (2020)

Orbilia carnegieae Baral \& G. Marson, Monogr. Orbiliomycetes: 792 (2020)

Orbilia caudimaeandrina Baral \& G. Marson, Monogr. Orbiliomycetes: 1168 (2020)

Orbilia caulicola Baral \& G. Marson, Monogr. Orbiliomycetes: 803 (2020)

Orbilia cercidicola Baral, G. Marson \& E.S. Popov, Monogr. Orbiliomycetes: 501 (2020)

Orbilia cercocarpi Baral \& G. Marson, Monogr. Orbiliomycetes: 1213 (2020)

Orbilia cisti Baral \& E. Weber, Monogr. Orbiliomycetes: 1217 (2020)

Orbilia clavipisca Baral \& G. Marson, Monogr. Orbiliomycetes: 1283 (2020)

Orbilia clavuliaristata Baral \& E. Weber, Monogr. Orbiliomycetes: 706 (2020)

Orbilia clavuliformis Baral \& G. Marson, Monogr. Orbiliomycetes: 680 (2020)

Orbilia cocois Baral, Monogr. Orbiliomycetes: 1474 (2020)

Orbilia colombiana Baral \& Priou, Monogr. Orbiliomycetes: 679 (2020)

Orbilia commarosa Baral \& G. Marson, Monogr. Orbiliomycetes: 1195 (2020)

Orbilia concoloris Baral \& G. Marson, Monogr. Orbiliomycetes: 672 (2020)

Orbilia coniferarum Baral \& E. Weber, Monogr. Orbiliomycetes: 1163 (2020)

Orbilia coronohesperidea Baral, Monogr. Orbiliomycetes: 583 (2020)

Orbilia crenatofalcata Baral \& Tello, Monogr. Orbiliomycetes: 883 (2020)

*Orbilia crenatonemaspora Baral \& Hong Y. Su, Monogr. Orbiliomycetes: 862 (2020)

Orbilia crenatovinosa Baral \& Friebes, Monogr. Orbiliomycetes: 643 (2020) 
Orbilia cryptogena Baral \& G. Marson, Monogr. Orbiliomycetes: 662 (2020)

Orbilia cucumispora Baral \& G. Marson, Monogr. Orbiliomycetes: 494 (2020)

Orbilia cupressi Baral \& E. Weber, Monogr. Orbiliomycetes: 1479 (2020)

Orbilia cupularis Baral \& G. Marson, Monogr. Orbiliomycetes: 1253 (2020)

Orbilia curvatimyriella Baral \& G. Marson, Monogr. Orbiliomycetes: 1234 (2020)

Orbilia curvatinavajoana Baral \& G. Marson, Monogr. Orbiliomycetes: 575 (2020)

Orbilia curvatiobliqua Baral, Monogr. Orbiliomycetes: 761 (2020)

Orbilia curvatitrapeziformis Baral, G. Marson \& Quijada, Monogr. Orbiliomycetes: 1101 (2020)

Orbilia curvativitalbae Baral \& G. Marson, Monogr. Orbiliomycetes: 1193 (2020)

Orbilia cylindrosoma Baral, E. Weber \& G. Marson, Monogr. Orbiliomycetes: 1068 (2020)

Orbilia cylindrospora Baral, E. Weber \& G. Marson, Monogr. Orbiliomycetes: 1314 (2020)

Orbilia dalmatica Baral, Monogr. Orbiliomycetes: 1332 (2020)

Orbilia delphinus Baral \& G. Marson, Monogr. Orbiliomycetes: 1105 (2020)

Orbilia denticulata Baral, R. Galán \& G. Marson, Monogr. Orbiliomycetes: 798 (2020)

Orbilia desertorum Baral \& Priou, Monogr. Orbiliomycetes: 1507 (2020)

Orbilia dixiensis Baral \& G. Marson, Monogr. Orbiliomycetes: 1177 (2020)

Orbilia edulis Baral \& E. Weber, Monogr. Orbiliomycetes: 1102 (2020)

Orbilia epilobii Baral \& E. Weber, Monogr. Orbiliomycetes: 1468 (2020)

Orbilia eremaeae Baral, Monogr. Orbiliomycetes: 1285 (2020)

Orbilia fabacearum Baral \& G. Marson,
Monogr. Orbiliomycetes: 1423 (2020)

Orbilia farnesianae Baral, Monogr. Orbiliomycetes: 923 (2020)

Orbilia ficicola G. Marson, Baral \& E. Weber, Monogr. Orbiliomycetes: 892 (2020)

Orbilia filiformis Baral, E. Weber \& P. Perz, Monogr. Orbiliomycetes: 1083 (2020)

Orbilia fimbriata Baral \& G. Marson, Monogr. Orbiliomycetes: 728 (2020)

Orbilia flavovacuolata Baral \& E. Weber, Monogr. Orbiliomycetes: 1639 (2020)

Orbilia flexisoma Baral \& G. Marson, Monogr. Orbiliomycetes: 1095 (2020)

Orbilia floridensis Baral, Monogr. Orbiliomycetes: 927 (2020)

Orbilia foliicola Baral \& E. Weber, Monogr. Orbiliomycetes: 482 (2020)

Orbilia frangulae G. Marson, Baral \& E. Weber, Monogr. Orbiliomycetes: 1345 (2020)

Orbilia fraxini Baral \& P. Perz, Monogr. Orbiliomycetes: 1480 (2020)

Orbilia frullaniae Baral \& Priou, Monogr. Orbiliomycetes: 704 (2020)

*Orbilia fusiformis Baral \& Hong Y. Su, Monogr. Orbiliomycetes: 998 (2020)

Orbilia geijerae Baral, Monogr. Orbiliomycetes: 1274 (2020)

Orbilia gemma Baral \& G. Marson, Monogr. Orbiliomycetes: 934 (2020)

Orbilia graminis Baral \& G. Marson, Monogr. Orbiliomycetes: 750 (2020)

Orbilia gregorii Baral, Monogr. Orbiliomycetes: 1289 (2020)

Orbilia guyanensis Baral, Monogr. Orbiliomycetes: 1380 (2020)

Orbilia halimi Baral \& E. Weber, Monogr. Orbiliomycetes: 1091 (2020)

Orbilia helicoobliqua Baral \& G. Marson, Monogr. Orbiliomycetes: 760 (2020)

Orbilia helicovinosa Baral, Monogr. Orbiliomycetes: 630 (2020)

*Orbilia hoana Baral, Hong Y. Su \& Y.C. Su, 
Monogr. Orbiliomycetes: 987 (2020)

Orbilia idahoensis Baral \& G. Marson, Monogr. Orbiliomycetes: 1094 (2020)

Orbilia jacaensis Baral, Priou \& E. Weber, Monogr. Orbiliomycetes: 1133 (2020)

*Orbilia jinguangsiensis Baral \& Hong Y. Su, Monogr. Orbiliomycetes: 1002 (2020)

Orbilia jurana Baral, Monogr. Orbiliomycetes: 1190 (2020)

Orbilia kingsiana Baral \& G. Marson, Monogr. Orbiliomycetes: 793 (2020)

Orbilia lacrimispora Baral \& G. Marson, Monogr. Orbiliomycetes: 1053 (2020)

Orbilia lamarcheae Baral, Monogr. Orbiliomycetes: 1485 (2020)

Orbilia lanternae Baral \& G. Marson, Monogr. Orbiliomycetes: 511 (2020)

Orbilia lentiformis Baral \& G. Marson, Monogr. Orbiliomycetes: 470 (2020)

Orbilia lilacina Baral \& G. Marson, Monogr. Orbiliomycetes: 1265 (2020)

Orbilia liliputiana Baral, Quijada \& E. Weber, Monogr. Orbiliomycetes: 1331 (2020)

Orbilia livistonae Baral, Monogr. Orbiliomycetes: 1120 (2020)

Orbilia lobeliae Baral, Monogr. Orbiliomycetes: 899 (2020)

Orbilia macroasca Baral \& G. Marson, Monogr. Orbiliomycetes: 507 (2020)

Orbilia macrocarpa Baral \& G. Marson, Monogr. Orbiliomycetes: 568 (2020)

Orbilia macrodelphinus Baral \& G. Marson, Monogr. Orbiliomycetes: 1113 (2020)

Orbilia macrohesperidea Baral \& G. Marson, Monogr. Orbiliomycetes: 590 (2020)

Orbilia macroserpens Baral \& G. Marson, Monogr. Orbiliomycetes: 1215 (2020)

Orbilia macrotrapeziformis Baral \& E. Weber, Monogr. Orbiliomycetes: 1098 (2020)

Orbilia maeandrina Baral \& G. Marson, Monogr. Orbiliomycetes: 1075 (2020)

Orbilia magnifica Baral \& G. Marson, Monogr. Orbiliomycetes: 509 (2020)
Orbilia mali Baral, Monogr. Orbiliomycetes: 781 (2020)

Orbilia mammifera Baral, Monogr. Orbiliomycetes: 1043 (2020)

Orbilia martinicensis Baral, Priou \& Lechat, Monogr. Orbiliomycetes: 995 (2020)

Orbilia megahesperidea Baral \& G. Marson, Monogr. Orbiliomycetes: 591 (2020)

Orbilia megaocculta Baral, Monogr. Orbiliomycetes: 727 (2020)

Orbilia menageshae Baral \& U. Lindem., Monogr. Orbiliomycetes: 1558 (2020)

Orbilia mesaverdiana Baral \& G. Marson, Monogr. Orbiliomycetes: 1148 (2020)

Orbilia microlentiformis Baral, Monogr. Orbiliomycetes: 1300 (2020)

Orbilia microserpens Baral, Monogr. Orbiliomycetes: 1223 (2020)

Orbilia microsoma Baral, G. Marson \& E. Weber, Monogr. Orbiliomycetes: 1298 (2020)

Orbilia mirabilis Baral, G. Marson \& E. Weber, Monogr. Orbiliomycetes: 1311 (2020)

Orbilia mongolica Baral, Monogr. Orbiliomycetes: 1041 (2020)

Orbilia montigena Baral \& E. Weber, Monogr. Orbiliomycetes: 592 (2020)

Orbilia multiaustraliensis Baral \& G. Marson, Monogr. Orbiliomycetes: 563 (2020)

Orbilia multiaustrocylindrica Baral, Monogr. Orbiliomycetes: 1321 (2020)

Orbilia multicercocarpi Baral \& G. Marson, Monogr. Orbiliomycetes: 1214 (2020)

Orbilia multicreosoteris Baral \& G. Marson, Monogr. Orbiliomycetes: 1275 (2020)

Orbilia multicurvula Baral \& G. Marson, Monogr. Orbiliomycetes: 636 (2020)

Orbilia multidelphinus Baral, Monogr. Orbiliomycetes: 1116 (2020)

Orbilia multigambelii Baral \& G. Marson, Monogr. Orbiliomycetes: 1209 (2020)

Orbilia multihamulata Baral \& G. Marson, Monogr. Orbiliomycetes: 1054 (2020) 
Orbilia multimaeandrina Baral \& G. Marson, Monogr. Orbiliomycetes: 1078 (2020)

Orbilia multinanosoma Baral, Monogr. Orbiliomycetes: 1308 (2020)

Orbilia multiphanosoma Baral \& G. Marson, Monogr. Orbiliomycetes: 515 (2020)

Orbilia multiserpens Baral \& G. Marson, Monogr. Orbiliomycetes: 1227 (2020)

Orbilia multitrapezoidea Baral \& G. Marson, Monogr. Orbiliomycetes: 1117 (2020)

Orbilia multiurosperma Baral \& G. Marson, Monogr. Orbiliomycetes: 1137 (2020)

Orbilia multivinosa Baral \& G. Marson, Monogr. Orbiliomycetes: 633 (2020)

Orbilia multivirgula Baral \& G. Marson, Monogr. Orbiliomycetes: 1236 (2020)

Orbilia myriella Baral \& G. Marson, Monogr. Orbiliomycetes: 1233 (2020)

Orbilia myrioauris Baral \& G. Marson, Monogr. Orbiliomycetes: 670 (2020)

Orbilia myrioaustraliensis Baral \& E. Weber, Monogr. Orbiliomycetes: 564 (2020)

Orbilia myrioeuonymi Baral \& G. Marson, Monogr. Orbiliomycetes: 538 (2020)

Orbilia myrioflexa Baral, Monogr. Orbiliomycetes: 1211 (2020)

Orbilia myriofusiclava Baral \& G. Marson, Monogr. Orbiliomycetes: 653 (2020)

Orbilia myriofusoidea Baral, Monogr. Orbiliomycetes: 1236 (2020)

Orbilia myriohesperidea Baral, Monogr. Orbiliomycetes: 589 (2020)

Orbilia myriolentiformis Baral \& G. Marson, Monogr. Orbiliomycetes: 494 (2020)

Orbilia myriolilacina Baral \& G. Marson, Monogr. Orbiliomycetes: 1269 (2020)

Orbilia myriomuscula Baral \& G. Marson, Monogr. Orbiliomycetes: 666 (2020)

Orbilia myrionamibica Baral, Monogr. Orbiliomycetes: 1239 (2020)

Orbilia myrionanosoma Baral \& G. Marson, Monogr. Orbiliomycetes: 1309 (2020)

Orbilia myrioobliqua Baral \& G. Marson,
Monogr. Orbiliomycetes: 753 (2020)

Orbilia myrioolneyae Baral \& G. Marson, Monogr. Orbiliomycetes: 1231 (2020)

Orbilia myriophanosoma Baral \& G. Marson, Monogr. Orbiliomycetes: 517 (2020)

Orbilia myriopseudoregalis Baral \& G. Marson, Monogr. Orbiliomycetes: 1313 (2020)

Orbilia myriosphaera Baral \& E. Weber, Monogr. Orbiliomycetes: 523 (2020)

Orbilia myriourosperma Baral \& G. Marson, Monogr. Orbiliomycetes: 1141 (2020)

Orbilia namibica Baral \& G. Marson, Monogr. Orbiliomycetes: 1238 (2020)

Orbilia nanosperma Baral \& G. Marson, Monogr. Orbiliomycetes: 737 (2020)

Orbilia naumburgensis Baral \& E. Weber, Monogr. Orbiliomycetes: 1640 (2020)

Orbilia navajoana Baral \& G. Marson, Monogr. Orbiliomycetes: 571 (2020)

Orbilia navicularis S. Tello, Baral \& E. Weber, Monogr. Orbiliomycetes: 936 (2020)

*Orbilia nemaspora Baral, Bin Liu, A.I. Romero, Healy \& Pfister, Monogr. Orbiliomycetes: 858 (2020)

*Orbilia neocomma Baral, S.F. Li, J.W. Guo, Z.F. Yu \& G. Marson, Monogr. Orbiliomycetes: 817 (2020)

Orbilia nothoaprilis Baral, Monogr. Orbiliomycetes: 640 (2020)

Orbilia nothovinosa Baral, Monogr. Orbiliomycetes: 627 (2020)

Orbilia obtusispora Baral \& E. Weber, Monogr. Orbiliomycetes: 1061 (2020)

Orbilia ocellata Baral, G. Marson \& E. Weber, Monogr. Orbiliomycetes: 476 (2020)

Orbilia octocercocarpi Baral \& E. Weber, Monogr. Orbiliomycetes: 1212 (2020)

Orbilia octocorculispora $\mathrm{R}$. Tena, Baral \& E. Weber, Monogr. Orbiliomycetes: 660 (2020) Orbilia octoserpentina Baral \& G. Marson, Monogr. Orbiliomycetes: 1122 (2020)

Orbilia octosporoides Baral \& G. Marson, 
Monogr. Orbiliomycetes: 1280 (2020)

Orbilia olivacea Baral \& G. Marson, Monogr. Orbiliomycetes: 1290 (2020)

Orbilia ophiosoma Baral \& G. Marson, Monogr. Orbiliomycetes: 1050 (2020)

Orbilia osteospermae Baral \& G. Marson, Monogr. Orbiliomycetes: 1082 (2020)

Orbilia ovalis Baral \& G. Marson, Monogr. Orbiliomycetes: 498 (2020)

Orbilia ovoidea Baral \& G. Marson, Monogr. Orbiliomycetes: 1254 (2020)

Orbilia palmicola Baral, Monogr. Orbiliomycetes: 567 (2020)

Orbilia paloverdensis Baral \& G. Marson, Monogr. Orbiliomycetes: 1169 (2020)

Orbilia paracaudata Baral \& G. Marson, Monogr. Orbiliomycetes: 925 (2020)

Orbilia paracylindrospora Baral \& E. Weber, Monogr. Orbiliomycetes: 1343 (2020)

Orbilia paradoxoides Baral, Monogr. Orbiliomycetes: 831 (2020)

Orbilia paramontigena Baral, Bometón \& E. Weber, Monogr. Orbiliomycetes: 599 (2020)

Orbilia paraobliqua Baral \& G. Marson, Monogr. Orbiliomycetes: 757 (2020)

Orbilia paravitalbae Baral, E. Weber, Priou \& Tena, Monogr. Orbiliomycetes: 910 (2020)

Orbilia parviclava Baral, Monogr. Orbiliomycetes: 688 (2020)

Orbilia patellarioides Baral \& G. Marson, Monogr. Orbiliomycetes: 461 (2020)

Orbilia phanosoma Baral \& G. Marson, Monogr. Orbiliomycetes: 514 (2020)

Orbilia phragmitis Baral, Monogr. Orbiliomycetes: 1093 (2020)

Orbilia pileosoma Baral \& G. Marson, Monogr. Orbiliomycetes: 468 (2020)

Orbilia pisciculus Baral \& G. Marson, Monogr. Orbiliomycetes: 1282 (2020)

Orbilia pleioalbidorosea Baral, Monogr. Orbiliomycetes: 943 (2020)

Orbilia pleioaustraliensis Baral \& G. Marson, Monogr. Orbiliomycetes: 560 (2020)
Orbilia pleioaustrocylindrica Baral, G. Marson \& E. Weber, Monogr. Orbiliomycetes: 1319 (2020)

Orbilia pleiocoronohesperidea Baral \& G. Marson, Monogr. Orbiliomycetes: 586 (2020)

Orbilia pleiocrescens Baral \& G. Marson, Monogr. Orbiliomycetes: 1227 (2020)

Orbilia pleioeuonymi Baral, G. Marson \& Priou, Monogr. Orbiliomycetes: 529 (2020)

Orbilia pleiogambelii Baral, Monogr. Orbiliomycetes: 1205 (2020)

Orbilia pleiohesperidea Baral, Monogr. Orbiliomycetes: 582 (2020)

Orbilia pleiolentiformis Baral \& G. Marson, Monogr. Orbiliomycetes: 484 (2020)

Orbilia pleiomesaverdiana Baral \& G. Marson, Monogr. Orbiliomycetes: 1149 (2020)

Orbilia pleiomicrosoma Baral \& G. Marson, Monogr. Orbiliomycetes: 1301 (2020)

Orbilia pleionavajoana Baral \& G. Marson, Monogr. Orbiliomycetes: 573 (2020)

Orbilia pleioobtusispora Baral, Monogr. Orbiliomycetes: 1067 (2020)

Orbilia pleioquaestiformis Baral \& G. Marson, Monogr. Orbiliomycetes: 1157 (2020)

Orbilia pleioserpens Baral \& G. Marson, Monogr. Orbiliomycetes: 1219 (2020)

Orbilia pleiostomachia Baral, Monogr. Orbiliomycetes: 519 (2020)

Orbilia pleioungulata Baral, Quijada \& R. Tena, Monogr. Orbiliomycetes: 1184 (2020) Orbilia pleiourosperma Baral \& G. Marson, Monogr. Orbiliomycetes: 1136 (2020)

Orbilia pleiovinosa Baral \& G. Marson, Monogr. Orbiliomycetes: 632 (2020)

Orbilia pleiovitalbae Baral, Monogr. Orbiliomycetes: 1191 (2020)

Orbilia pleistoeuonymi Baral \& P. Perz, Monogr. Orbiliomycetes: 539 (2020)

Orbilia pleistolilacina Baral, G. Marson \& E. Weber, Monogr. Orbiliomycetes: 1271 (2020) 
Orbilia pleistoobliqua Baral \& G. Marson, Monogr. Orbiliomycetes: 755 (2020)

Orbilia pleistosphaera S. Tello \& Baral, Monogr. Orbiliomycetes: 526 (2020)

Orbilia pleistovitalbae Baral \& G. Marson, Monogr. Orbiliomycetes: 1192 (2020)

Orbilia plurilentiformis Baral \& G. Marson, Monogr. Orbiliomycetes: 492 (2020)

Orbilia plurililacina Baral, G. Marson \& E. Weber, Monogr. Orbiliomycetes: 1267 (2020)

Orbilia pluristomachia Baral \& G. Marson, Monogr. Orbiliomycetes: 520 (2020)

Orbilia plurivacuolata Baral, G. Marson \& E. Weber, Monogr. Orbiliomycetes: 1347 (2020)

Orbilia poitevinica Baral, Monogr. Orbiliomycetes: 913 (2020)

Orbilia ponderosae Baral \& G. Marson, Monogr. Orbiliomycetes: 1286 (2020)

Orbilia pseudeuphorbiae Baral, Quijada \& Beltran-Tej., Monogr. Orbiliomycetes: 1476 (2020)

Orbilia pseudoaristata Baral \& G. Marson, Monogr. Orbiliomycetes: 947 (2020)

Orbilia pseudocylindrospora Baral \& G. Marson, Monogr. Orbiliomycetes: 798 (2020)

Orbilia pseudoflagellispora Baral \& G. Marson, Monogr. Orbiliomycetes: 951 (2020)

*Orbilia pseudopolybrocha Z.F. Yu \& M. Qiao, Int. J. Syst. Evol. Microbiol. 70 (4): 2666 (2020)

Orbilia pubescens Baral \& G. Marson, Monogr. Orbiliomycetes: 936 (2020)

Orbilia purshiae Baral \& G. Marson, Monogr. Orbiliomycetes: 466 (2020)

Orbilia puyae Baral \& Priou, Monogr. Orbiliomycetes: 796 (2020)

Orbilia pyrenaica Baral, J.P. Priou \& E. Weber, Monogr. Orbiliomycetes: 1100 (2020)

Orbilia quaestiformis Baral \& G. Marson,
Monogr. Orbiliomycetes: 1151 (2020)

Orbilia quercus-ilicis Baral \& E. Weber, Monogr. Orbiliomycetes: 1162 (2020)

Orbilia rehmii Baral, Monogr. Orbiliomycetes: 904 (2020)

Orbilia rhamni Baral \& Priou, Monogr. Orbiliomycetes: 1483 (2020)

Orbilia rubrovacuolata Baral, Priou \& E. Weber, Monogr. Orbiliomycetes: 1587 (2020)

Orbilia saguarina Baral \& G. Marson, Monogr. Orbiliomycetes: 1221 (2020)

Orbilia sarcobati Baral \& G. Marson, Monogr. Orbiliomycetes: 1287 (2020)

Orbilia sarothamni Baral, Monogr. Orbiliomycetes: 668 (2020)

Orbilia scandens Baral \& G. Marson, Monogr. Orbiliomycetes: 1042 (2020)

Orbilia sedonensis Baral \& G. Marson, Monogr. Orbiliomycetes: 1142 (2020)

Orbilia siculispora Baral, E. Weber, Friebes \& G. Marson, Monogr. Orbiliomycetes: 886 (2020)

Orbilia solidaginis Baral, Monogr. Orbiliomycetes: 1048 (2020)

Orbilia somedana Baral \& E. Weber, Monogr. Orbiliomycetes: 904 (2020)

Orbilia sonorensis Baral \& G. Marson, Monogr. Orbiliomycetes: 1145 (2020)

Orbilia spathulata Baral \& G. Marson, Monogr. Orbiliomycetes: 1112 (2020)

Orbilia spermoides Baral \& G. Marson, Monogr. Orbiliomycetes: 734 (2020)

Orbilia sphaerospora Baral \& G. Marson, Monogr. Orbiliomycetes: 1246 (2020)

Orbilia spirillospora Baral \& G. Marson, Monogr. Orbiliomycetes: 866 (2020)

Orbilia spirospora Baral \& E. Weber, Monogr. Orbiliomycetes: 1384 (2020)

Orbilia stansburyanae Baral, Monogr. Orbiliomycetes: 1272 (2020)

Orbilia stilbospora Baral, Monogr. Orbiliomycetes: 807 (2020) 
Orbilia subalbovinosa Baral \& G. Marson, Monogr. Orbiliomycetes: 946 (2020)

Orbilia subaristata Baral, G. Marson \& Matočec, Monogr. Orbiliomycetes: 719 (2020)

Orbilia subclavuliformis Baral, E. Weber \& Priou, Monogr. Orbiliomycetes: 690 (2020)

Orbilia subcryptogena Baral \& G. Marson, Monogr. Orbiliomycetes: 665 (2020)

Orbilia subcylindrospora Baral \& E. Weber, Monogr. Orbiliomycetes: 1277 (2020)

Orbilia subdelphinus Baral, Monogr. Orbiliomycetes: 1111 (2020)

Orbilia suberis Baral \& Galán, Monogr. Orbiliomycetes: 895 (2020)

Orbilia subfabacearum Baral \& G. Marson, Monogr. Orbiliomycetes: 1426 (2020)

Orbilia subocellata Baral \& E. Weber, Monogr. Orbiliomycetes: 481 (2020)

Orbilia subovoidea Baral, Matočec \& E. Weber, Monogr. Orbiliomycetes: 1257 (2020)

Orbilia subsiculispora G. Marson \& Baral, Monogr. Orbiliomycetes: 890 (2020)

Orbilia subsphaerospora Baral, Monogr. Orbiliomycetes: 1249 (2020)

Orbilia subtrapeziformis Baral, E. Weber \& G. Marson, Monogr. Orbiliomycetes: 1088 (2020)

Orbilia subuliformis Baral, Monogr. Orbiliomycetes: 1001 (2020)

Orbilia subulivinosa G. Marson, Baral \& E. Weber, Monogr. Orbiliomycetes: 634 (2020)

Orbilia subvinosa G. Marson, Baral \& E. Weber, Monogr. Orbiliomycetes: 616 (2020)

Orbilia subvitalbae Baral \& E. Weber, Monogr. Orbiliomycetes: 1179 (2020)

*Orbilia tenuispora E. Weber, Baral, Hong Y. Su, M.L. Wu, Y.C. Su \& Bin Liu, Monogr. Orbiliomycetes: 1007 (2020)

*Orbilia tonghaiensis Z.F. Yu, Baral \& E. Weber, Int. J. Syst. Evol. Microbiol. 70 (4): 2669 (2020)
Orbilia trapeziformis Baral \& G. Marson, Monogr. Orbiliomycetes: 1055 (2020)

Orbilia tremuloidis Baral \& E. Weber, Monogr. Orbiliomycetes: 512 (2020)

Orbilia triangulispora Baral, Monogr. Orbiliomycetes: 744 (2020)

Orbilia ungulata Baral, G. Marson \& Matočec, Monogr. Orbiliomycetes: 1180 (2020)

Orbilia urosperma Baral \& G. Marson, Monogr. Orbiliomycetes: 1135 (2020)

Orbilia velutina Baral \& G. Marson, Monogr. Orbiliomycetes: 638 (2020)

Orbilia vermiculati Baral \& G. Marson, Monogr. Orbiliomycetes: 1166 (2020)

Orbilia vibrioides Baral, Priou \& G. Marson, Monogr. Orbiliomycetes: 747 (2020)

Orbilia wannerooensis Baral \& G. Marson, Monogr. Orbiliomycetes: 1104 (2020)

Orbilia xanthoflexa Baral, Pfister \& Healy, Monogr. Orbiliomycetes: 1442 (2020)

Orbilia yuccae Baral \& G. Marson, Monogr. Orbiliomycetes: 1291 (2020)

*Oxydothis phoenicis S.N. Zhang, K.D. Hyde \& J.K. Liu, Mycosphere 11 (1): 770 (2020)

*Pachyphlodes atropurpurea J.W. Liu, S.P. Wan \& F.Q. Yu, Mycologia 464 (1): 87 (2020)

*Pachyphlodes excavata J.W. Liu, S.P. Wan \& F.Q. Yu, Phytotaxa 464 (1): 89 (2020)

Paecilomyces penicilliformis Jurjević \& Hubka, Persoonia 44: 431 (2020)

Palmeiromyces chamaeropicola D.R.S. Pereira \& A.J.L. Phillips, Phytopath. Mediterr. 59 (2): 360 (2020)

*Panaeolus axfordii Y.W. Hu, S.C. Karunarathna, P.E. Mortimer \& J.C. Xu, Phytotaxa 434 (1): 26 (2020)

Papiliotrema baii Yurkov, M.A. Guerreiro \& Á. Fonseca ex Yurkov, Stud. Mycol. 96: 135 (2020)

Papiliotrema frias V. de García, Zalar, Brizzio, Gunde-Cim. \& van Broock ex Yurkov, Stud. Mycol. 96: 135 (2020) 
Papiliotrema hoabinhensis D.T. Luong, M. Takash., Ty, Dung \& Nakase ex Yurkov, Stud. Mycol. 96: 135 (2020)

*Papiliotrema japonica J.P. Samp., Fonseca \& Fell ex Xin Zhan Liu, F.Y. Bai, M. Groenew. \& Boekhout, Stud. Mycol. 96: 135 (2020)

*Papiliotrema terrestris Crestani, Landell, Faganello, Vainstein, Vishniac \& P. Valente ex Xin Zhan Liu, F.Y. Bai, M. Groenew. \& Boekhout, Stud. Mycol. 96: 135 (2020)

*Papiliotrema wisconsinensis $\mathrm{K}$. Sylvester, Q.M. Wang \& Hittinger ex Xin Zhan Liu, F.Y. Bai, M. Groenew. \& Boekhout, Stud. Mycol. 96: 136 (2020)

*Parabambusicola aquatica W. Dong, H. Zhang \& K.D. Hyde, Fungal Diversity 10.1007/s13225-020-00463-5: 161 (2020)

*Paraboeremia rekkeri Hern.-Restr., L.W. Hou, L. Cai \& Crous, MycoKeys 65: 79 (2020)

*Paraboeremia taiwanensis H.A. Ariyaw., W.Y. Chuang \& J.I. Yang, Mycol. Progr. 19 (1): 102 (2020)

*Paraboeremia truiniorum Hern.-Restr., L.W. Hou, L. Cai \& Crous, MycoKeys 65: 81 (2020)

Paraconiothyrium camelliae H.A. Ariyaw., I. Tsai \& Thambug., Scientific Reports 10 (no. 12762): 16 (2020)

Paraconiothyrium iridis Crous \& Akulov, Fungal Systematics and Evolution 6: 210 (2020)

Paraconiothyrium salinum M. Gonçalves \& A. Alves, Int. J. Syst. Evol. Microbiol. 10.1099/ijsem.0.004410: 7 (2020)

*Paracremonium apiculatum Z.F. Zhang \& L. Cai, Fungal Diversity 10.1007/s13225-02000453-7: 79 (2020)

*Paracremonium ellipsoideum Z.F. Zhang \& L. Cai, Fungal Diversity 10.1007/s13225020-00453-7: 80 (2020)

*Parafuscosporella aquatica H. Yang \& H. Zhang, Phytotaxa 441 (1): 24 (2020)
*Parafuscosporella pyriformis $\mathrm{H}$. Yang, W. Dong \& H. Zhang, Phytotaxa 441 (1): 27 (2020)

Paragaeumannomyces abietinus Réblová, J. Fourn. \& A.N. Mill., MycoKeys 74: 21 (2020)

Paragaeumannomyces elegans Réblová \& A.N. Mill., MycoKeys 74: 26 (2020)

Paragaeumannomyces granulatus Réblová \& A.N. Mill., MycoKeys 74: 29 (2020)

Paragaeumannomyces sabinianus Réblová \& A.N. Mill., MycoKeys 74: 35 (2020)

Paragaeumannomyces smokiensis Réblová \& A.N. Mill., MycoKeys 74: 38 (2020)

Paraleptospora chromolaenae Mapook \& K.D. Hyde, Fungal Diversity 101: 76 (2020)

Paraleptospora chromolaenicola Mapook \& K.D. Hyde, Fungal Diversity 101: 77 (2020)

Paraloratospora camporesii Bundhun, Jeewon \& K.D. Hyde, Fungal Diversity 100: 105 (2020)

Paralulworthia gigaspora A. Poli, E. Bovio, L. Ranieri, G.C. Varese \& V. Prigione, Frontiers in Microbiology 11 (no. 933): 9 (2020)

Paralulworthia posidoniae A. Poli, E. Bovio, L. Ranieri, G.C. Varese \& V. Prigione, Frontiers in Microbiology 11 (no. 933): 9 (2020)

*Paramicrosphaeropsis ellipsoidea L.W. Hou, L. Cai \& Crous, Stud. Mycol. 96: 376 (2020) *Paramonodictys solitarius N.G. Liu, K.D. Hyde \& J.K. Liu, Fungal Diversity 100: 91 (2020)

Paramycetinis austrobrevipes R.H. Petersen, Mycotaxon 135: 9 (2020)

Paramycetinis caulocystidiatus R.H. Petersen, Mycotaxon 135: 21 (2020)

Paramyrothecium pituitipietianum Crous, Persoonia 45: 313 (2020)

Paraophiobolus torilicola Brahmanage, Camporesi \& K.D. Hyde, Mycosphere 11: 2515 (2020) 
Paraphaeosphaeria camelliae H.A. Ariyaw., I. Tsai \& Thambug., Scientific Reports 10 (no. 12762): 15 (2020)

*Paraphaeosphaeria hydei Z.F. Zhang \& L. Cai, Fungal Diversity 10.1007/s13225-02000453-7: 47 (2020)

Paraphoma melnikii Gomzhina \& Gasich, Mycol. Progr. 19: 192 (2020)

Paraphyton cutaneum Hubka, Kucerova, Gibas, Kubátová \& Hamal, Persoonia 44: 433 (2020)

Pararoussoella quercina Crous \& Akulov, Fungal Systematics and Evolution 6: 211 (2020)

Parasarocladium tasmanniae Crous, Persoonia 45: 283 (2020)

Parastagonospora dactylidicola Brahmanage, Camporesi \& K.D. Hyde, Mycosphere 11: 2512 (2020)

Parasulcatispora clematidis Phukhams. \& K.D. Hyde, Fungal Diversity 10.1007/s13225-020-00448-4: 121 (2020)

Parathyridaria clematidis Phukhams., Camporesi \& K.D. Hyde, Fungal Diversity 10.1007/s13225-020-00448-4: 129 (2020)

Parathyridaria flabelliae E. Bovio, A. Poli, Prigione \& Varese, Diversity 12 (4, no. 144): 10 (2020)

Parathyridaria serratifoliae Phukhams., Ertz, Gerstmans \& K.D. Hyde, Fungal Diversity 10.1007/s13225-020-00448-4: 131 (2020)

Parathyridaria tyrrhenica A. Poli, Prigione, E. Bovio \& Varese, Diversity 12 (4, no. 144): 9 (2020)

Parathyridaria virginianae Phukhams., Ertz, Gerstmans \& K.D. Hyde, Fungal Diversity 10.1007/s13225-020-00448-4: 132 (2020)

Parathyridariella dematiacea Prigione, A. Poli, E. Bovio \& Varese, Diversity 12 (4, no. 144): 8 (2020)

Parmelia rojoi A. Crespo, V.J. Rico \& Divakar, Lichenologist 52 (5): 372 (2020)

Parmotrema austromaculatum A.A. Spielm.
\& Marcelli, Plant and Fungal Systematics 65 (2): 418 (2020)

Parmotrema bifidum A.A. Spielm. \& Marcelli, Plant and Fungal Systematics 65 (2): 420 (2020)

Parmotrema clercianum A.A. Spielm., Plant and Fungal Systematics 65 (2): 426 (2020)

*Parvomorbus eucalypti Wen Wang \& S.F. Chen, Persoonia 45: 121 (2020)

*Parvomorbus guangdongensis Wen Wang \& S.F. Chen, Persoonia 45: 122 (2020)

Patellaria apiculatae Dayarathne \& K.D. Hyde, Mycosphere 11 (1): 82 (2020)

Patellaria chromolaenae Mapook \& K.D. Hyde, Fungal Diversity 101: 127 (2020)

Patellaria microspora Ekanayaka \& K.D. Hyde, Fungal Diversity 10.1007/s13225020-00462-6: 115 (2020)

Peltigera hydrophila W.R. Buck, J. Miadlikowska \& N. Magain, Plant and Fungal Systematics 65 (1): 212 (2020)

Peltigera serusiauxii Magain, Miadl., Goffinet $\&$ Ant. Simon, Plant and Fungal Systematics 65 (1): 143 (2020)

Penicillium labradorum Gibas, Wiederh., C. Sanders, Rothacker, E.R. Rogers \& Fales, Medical Mycology 58 (8): 1061 (2020)

Penicillium rotoruae O'Callahan \& Vaidya, Curr. Microbiol. 77: 4131 (2020)

Penicillium saanichanum Visagie, Assabgui \& Seifert, Persoonia 45: 373 (2020)

*Penicillium soli Doilom, C.F. Liao \& D. Pem, Frontiers in Microbiology 11 (no. 585215): 13 (2020)

Penicillium taurinense S. Prencipe, Houbraken \& D. Spadaro, Persoonia 44: 435 (2020)

Penicillium vallebormidaense Houbraken \& Di Piazza, Persoonia 45: 371 (2020)

Penicillium vascosobrinhous R.N. Barbosa \& J.D.P. Bezerra, Acta Bot. Brasilica 34 (2): 412 (2020)

*Peniophorella aspersa Yurchenko \& Sheng. 
H. Wu, Nova Hedwigia 111 (3-4): 481 (2020)

*Peniophorella cremea C.L. Zhao, Phytotaxa 464 (2): 179 (2020)

*Peniophorella crystallifera Yurchenko, Sheng H. Wu \& N. Maek., Nova Hedwigia 111 (3-4): 485 (2020)

*Peniophorella fissurata C.L. Zhao, Mycol. Progr. 19 (4): 400 (2020)

*Peniophorella reticulata Yurchenko \& Sheng.

H. Wu, Nova Hedwigia 111 (3-4): 488 (2020)

*Perenniporia eugeissonae $\mathrm{P}$. Du \& Chao G.

Wang, Phytotaxa 449 (1): 80 (2020)

*Perenniporia pseudotephropora Chao G. Wang \& F. Wu, MycoKeys 69: 56 (2020)

Perenniporia reflexa Ryvarden, Syn. Fung. 40: 104 (2020)

*Perenniporia subcorticola Chao G. Wang \& F. Wu, MycoKeys 69: 62 (2020)

Perichaena patagonica A. Ronikier \& Lado, Mycologia 112 (4): 766 (2020)

*Periconia palmicola J.F. Li \& Phookamsak, Fungal Diversity 100: 93 (2020)

Periconia salina Dayarathne \& E.B.G. Jones, Mycosphere 11 (1): 50 (2020)

Periconia verrucosa Phukhams., Ertz, Gerstmans \& K.D. Hyde, Fungal Diversity 10.1007/s13225-020-00448-4: 71 (2020)

Periplasma isogametum W.W. Martin \& A. Warren, Mycologia 112 (5): 998 (2020)

Peroneutypa indica Devadatha, V.V. Sarma \& E.B.G Jones, Mycosphere 11 (1): 145 (2020)

Peroneutypa polysporae Devadatha, V.V. Sarma \& E.B.G Jones, Mycosphere 11 (1): 146 (2020)

Peronospora choii Hoffmeister, W. Maier \& Thines, Fungal Systematics and Evolution 6: 45 (2020)

Peronospora salviae-pratensis Hoffmeister, W. Maier \& Thines, Fungal Systematics and Evolution 6: 45 (2020)

Pertusaria alloisidiosa A.W. Archer \& Elix, Australas. Lichenol. 86: 17 (2020)

Pertusaria copiofructa A.W. Archer \& Elix,
Australas. Lichenol. 86: 17 (2020)

Pertusaria macroides A.W. Archer \& Elix, Australas. Lichenol. 86: 17 (2020)

*Pestalotiopsis etonensis C. Lock, Vitelli, Holdom, Y.P. Tan \& R.G. Shivas, Persoonia 44: 437 (2020)

Pestalotiopsis kandelicola Norph., C.H. Kuo \& K.D. Hyde, Fungal Diversity 10.1007/s13225-020-00458-2: 16 (2020)

Pestalotiopsis pini A.C. Silva, E. Diogo \& H. Bragança, Forests 11 (8/850): 9 (2020)

*Pestalotiopsis rhizophorae Norphanphoun, T.C. Wen \& K.D. Hyde, Mycosphere 10 (1): 552 (2020)

Pestalotiopsis thailandica Norphanphoun, Doilom \& K.D. Hyde, Mycosphere 10 (1): 555 (2020)

Petchia siamensis Thanakitp., Mongkols. \& Luangsa-ard, Persoonia 44: 154 (2020)

Petrakia greenei Beenken, Andr. Gross \& Queloz, Mycol. Progr. 19 (5): 431 (2020)

Petrakia liobae Beenken, Andr. Gross \& Queloz, Mycol. Progr. 19 (5): 432 (2020)

*Pezicula italica W.J. Li, Camporesi \& K.D. Hyde, Fungal Diversity 100: 639 (2020)

Peziza granularis Donadini ex Van Vooren, Die Pilze Deutschlands 7: 56 (2020)

Peziza martinicensis Van Vooren \& F. Lopez, Ascomycete.org 12 (2): 58 (2020)

Peziza subvesiculosa Donadini \& Van Vooren, Cahiers de la FMBDS 7: 59 (2020)

Phacidiella alsophilae Crous, Persoonia 44: 319 (2020)

*Phacidium italicum W.J. Li, Camporesi \& K.D. Hyde, Fungal Diversity 100: 643 (2020) Phaeocandelabrum pseudocallisporum Careli \& Gusmão, Mycotaxon 135 (4): 760 (2020)

Phaeoclavulina alboapiculata Franchi \& M. Marchetti, Index Fungorum 467: 1 (2020)

Phaeoclavulina carovinacea Franchi \& M. Marchetti, Index Fungorum 467: 2 (2020)

Phaeoclavulina caroviridula Franchi \& M. Marchetti, Index Fungorum 467: 2 (2020) 
Phaeoclavulina coniferarum Franchi \& M. Marchetti, Index Fungorum 467: 3 (2020)

Phaeoclavulina minutispora Franchi \& M. Marchetti, Index Fungorum 467: 3 (2020)

Phaeoclavulina nigricans E. Campo, Franchi \& M. Marchetti, Index Fungorum 467: 4 (2020)

Phaeodothis mori D.S. Tennakoon, C.H. Kuo \& K.D. Hyde, Phytotaxa 428 (3): 247 (2020)

Phaeoplaca tortuca Søchting \& Bungartz, Plant and Fungal Systematics 65 (2): 546 (2020)

Phaeoseptum carolshearerianum Devadatha, V.V. Sarma \& E.B.G. Jones, Mycosphere 11 (1): 53 (2020)

Phaeoseptum hydei Wanas., Senwanna \& Mortimer, Phytotaxa 449 (2): 153 (2020)

Phaeoseptum manglicola Devadatha, V.V. Sarma \& E.B.G. Jones, Mycosphere 11 (1): 56 (2020)

Phaeosphaeriopsis beaucarneae D.S. Tennakoon, C.H. Kuo \& K.D. Hyde, MycoKeys 70: 75 (2020)

Phaeospora australiensis P.M. McCarthy \& Elix, Australas. Lichenol. 87: 48 (2020)

*Phaeotremella lactea Q.M. Wang, F.Y. Bai \& A.H. Li, Stud. Mycol. 96: 98 (2020)

*Phaeotremella ovata Q.M. Wang, F.Y. Bai \& A.H. Li, Stud. Mycol. 96: 98 (2020)

*Phaeotremella yunnanensis L.F. Fan, F. Wu \& Y.C. Dai, Fungal Diversity 10.1007/s13225-020-00461-7: 245 (2020)

Phaeoxyphiella australiana Abdollahz. \& Crous, Stud. Mycol. 95: 408 (2020)

Phaeoxyphiella phylicae Abdollahz. \& Crous, Stud. Mycol. 95: 408 (2020)

*Phaffia aurantiaca Q.M. Wang, F.Y. Bai \& A.H. Li, Stud. Mycol. 96: 103 (2020)

Phaffia australis David-Palma, Libkind, P. Gonç. \& J.P. Samp., Microorganisms 8 (11, no. 1651): 10 (2020)

Phaffia tasmanica David-Palma, Libkind, P. Gonç. \& J.P. Samp., Microorganisms 8 (11, no. 1651): 11 (2020)

*Phallus dongsun T.H. Li, T. Li, Chun Y. Deng, W.Q. Deng \& Zhu L. Yang, Phytotaxa 443 (1): 29 (2020)

*Phallus lutescens T.H. Li, T. Li \& W.Q. Deng, Phytotaxa 443 (1): 30 (2020)

*Phanerochaete burdsallii Y.L. Xu, Nakasone \& S.H. He, Mycosphere 11 (1): 1531 (2020) *Phanerochaete cinerea Y.L. Xu \& S.H. He, Mycosphere 11 (1): 1535 (2020)

*Phanerochaete hymenochaetoides Y.L. Xu \& S.H. He, Mycosphere 11 (1): 1537 (2020)

*Phanerochaete leptocystidiata Y.L. Xu \& S.H. He, Mycosphere 11 (1): 1538 (2020)

*Phanerochaete metuloidea Y.L. Xu \& S.H. He, Mycosphere 11 (1): 1540 (2020)

*Phanerochaete minor Y.L. Xu \& S.H. He, Mycosphere 11 (1): 1542 (2020)

*Phanerochaete sinensis Y.L. Xu, C.C. Chen \& S.H. He, Mycosphere 11 (1): 1543 (2020)

*Phanerochaete subrosea Y.L. Xu \& S.H. He, Mycosphere 11 (1): 1544 (2020)

*Phanerochaete yunnanensis Y.L. Xu \& S.H. He, Mycosphere 11 (1): 1546 (2020)

Phellinus guttiformis Tchotet, M.P.A. Coetzee, Rajchenb. \& Jol. Roux, Mycologia 112 (4): 735 (2020)

Phialemonium pulveris Crous \& Decock, Fungal Systematics and Evolution 6: 212 (2020)

Phialocephala amethystea Tanney \& Seifert, Stud. Mycol. 95: 343 (2020)

Phialocephala biguttulata Tanney \& Seifert, Stud. Mycol. 95: 345 (2020)

Phialocephala collarifera Tanney \& Seifert, Stud. Mycol. 95: 346 (2020)

Phialocephala helenae Tanney \& Seifert, Stud. Mycol. 95: 346 (2020)

Phialocephala melitaea Matočec, I. Kušan, Pošta, Tkalčec \& Mešić, Persoonia 45: 375 (2020)

Phialocephala vermiculata Tanney \& Seifert, Stud. Mycol. 95: 348 (2020) 
*Phialolunulospora vermispora Z.F. Yu \& R.F. Castañeda, MycoKeys 76: 23 (2020)

Phialophoropsis hubbardii C. Mayers, T.C. Harr., McNew \& Roeper, Mycologia 112 (6): 1125 (2020)

Phialophoropsis leachii C. Mayers, T.C. Harr., McNew \& Roeper, Mycologia 112 (6): 1125 (2020)

Phialophoropsis nunbergii C. Mayers \& T.C. Harr., Mycologia 112 (6): 1124 (2020)

*Phlebia fuscotuberculata C.L. Zhao, Mycol. Progr. 19: 761 (2020)

*Phlebia nigrodontea C.L. Zhao \& R.X. Huang, Phytotaxa 458 (3): 200 (2020)

*Phlebia tomentopileata C.L. Zhao, Mycol. Progr. 19: 762 (2020)

*Phlebia tongxiniana C.L. Zhao, Mycol. Progr. 19: 763 (2020)

*Phlebiopsis lacerata C.L. Zhao, Phytotaxa 440 (4): 274 (2020)

*Phlyctema coronillae W.J. Li, Camporesi \& K.D. Hyde, Fungal Diversity 100: 656 (2020)

Pholiotina pleurocystidiata A. Hausknecht \& I. Krisai-Greilhuber, Öst. Z. Pilzk. 28: 14 (2020)

Pholiotina pseudoampullaceocystis Karich, Boletus 41 (2): 102 (2020)

*Phomatodes pilosa L.W. Hou, L. Cai \& Crous, Stud. Mycol. 96: 381 (2020)

Phomatospora uniseriata Phukhams., M.V. de Bult \& K.D. Hyde, Fungal Diversity 10.1007/s13225-020-00448-4: 167 (2020)

*Phragmidium jiangxiense Y.M. Liang \& Y. Liu, Mycologia 112 (4): 747 (2020)

*Phragmidium leucoaecium Y.M. Liang \& Y. Liu, Mycologia 112 (4): 747 (2020)

Phycophthorum isakeiti Hassett, J. Eukary. Microbiol. 67: 482 (2020)

*Phylloporia alyxiae Sheng H. Wu, Mycol. Progr. 19 (8): 746 (2020)

*Phylloporia mori Sheng H. Wu, Mycol. Progr. 19 (8): 747 (2020)

*Phylloporia murrayae Sheng H. Wu, Mycol.
Progr. 19 (8): 748 (2020)

*Phylloporia rubiacearum Sheng $\mathrm{H}$. Wu, Mycol. Progr. 19 (8): 748 (2020)

Phyllopsora dodongensis S.Y. Kondr. \& Hur, Acta bot. hung. 62 (1-2): 85 (2020)

Phyllosticta rhizophorae Norph. \& K.D. Hyde, Mycosphere 11 (1): 2562 (2020)

*Phyllozyma aceris Q.M. Wang, F.Y. Bai \& A.H. Li, Stud. Mycol. 96: 113 (2020)

*Phyllozyma jiayinensis Q.M. Wang, F.Y. Bai \& A.H. Li, Stud. Mycol. 96: 113 (2020)

Physarum australiense S.L. Stephenson, Novozh. \& I.S. Prikhodko, Nov. sist. Niz. Rast. 54 (2): 400 (2020)

Physcia microphylla Aptroot \& M.F. Souza, Archive For Lichenology 20: 1 (2020)

Physcia occidentalis Essl. \& McCune, Bryologist 123 (2): 206 (2020)

Physcia rhizinata Essl. \& McCune, Bryologist 123 (2): 209 (2020)

Physciella neotropica M.F. Souza \& Aptroot, Archive For Lichenology 20: 3 (2020)

*Phytophthora acaciuvora T.Q. Pham, T.I. Burgess \& Q.N. Dang, Fungal Systematics and Evolution 6: 246 (2020)

Phytophthora alpina Bregant, Montecchio \& Linald., Forests 11 (8, no. 848): 8 (2020)

Phytophthora aquae-cooljarloo Mostowf. \& T.I. Burgess, Persoonia 45: 377 (2020)

Phytophthora aysenensis M. Zapata, M.C. Asenjo \& M. Gut., Persoonia 44: 439 (2020) *Phytophthora cathayensis C. MoralesRodríguez, Y. Wang \& A. Vannini, Fungal Systematics and Evolution 7: 102 (2020)

Phytophthora personensis Z.G. Abad, W. Gut. \& T.I. Burgess, Persoonia 44: 441 (2020)

Phytopythium paucipapillatum Langenhoven, W.J. Botha \& L. Mostert, Persoonia 45: 379 (2020)

*Piskurozyma fildesensis T.T. Zhang \& Li Y. Yu ex Yurkov, Stud. Mycol. 96: 136 (2020) *Piskurozyma taiwanensis Nakase, Tsuzuki \& M. Takash. ex Xin Zhan Liu, F.Y. Bai, M. 
Groenew. \& Boekhout, Stud. Mycol. 96: 136 (2020)

Pithoascus persicus Z. Tazik \& K. Rahnama, Mycoscience 61 (3): 149 (2020)

Placynthium glaciale Fryday \& T. Sprib., Lichenologist 52 (2): 114 (2020)

Plenodomus triseptatus Wijesinghe, Bulgakov \& K.D. Hyde, Fungal Diversity 100: 71 (2020)

Pleopunctum clematidis Phukhams., D.J. Bhat \& K.D. Hyde, Fungal Diversity 10.1007/s13225-020-00448-4: 73 (2020)

Pleurotheciella erumpens Réblová \& J. Fourn., Stud. Mycol. 95: 456 (2020)

Plicatura alba Henkel \& Ryvarden, Syn. Fung. 40: 106 (2020)

Pluteus atypicus E.F. Malysheva \& A.V. Alexandrova, Phytotaxa 461 (2): 93 (2020)

Pluteus brunneovelutinus E.F. Malysheva \& O.V. Morozova, Phytotaxa 461 (2): 104 (2020)

Pluteus castaneorugosus E.F. Malysheva \& A.V. Alexandrova, Phytotaxa 461 (2): 95 (2020)

Pluteus cutefractus Ferisin, Dovana \& Justo, Sydowia 71: 285 (2020)

Pluteus flavidus E.F. Malysheva \& A.V. Alexandrova, Phytotaxa 461 (2): 96 (2020)

Pluteus keselakii Ševčíková, P.-A. Moreau \& Borovička, Phytotaxa 432 (2): 184 (2020)

Pluteus olivaceofibrillosus E.F. Malysheva \& A.V. Alexandrova, Phytotaxa 461 (2): 84 (2020)

Pluteus pallidosquamulosus E.F. Malysheva \& A.V. Alexandrova, Phytotaxa 461 (2): 85 (2020)

Pluteus podospilloides E.F. Malysheva \& O.V. Morozova, Phytotaxa 461 (2): 100 (2020)

Pluteus pygmaeus E.F. Malysheva, Phytotaxa 461 (2): 102 (2020)

Pluteus squamulososulcatus E.F. Malysheva \& O.V. Morozova, Phytotaxa 461 (2): 99 (2020)
Pluteus tenebromarginatus G. Corriol, Bull. Féd. Assoc. Mycol. Méditerr. 57: 4 (2020)

Pluteus varius E.F. Malysheva, O.V. Morozova \& A.V. Alexandrova, Phytotaxa 461 (2): 89 (2020)

*Pneumocystis canis C. Weissenbacher-Lang, L. Ma, O.H. Cissé \& J.A. Kovacs, Index Fungorum 450: 1 (2020)

Poaceascoma filiforme Crous, Persoonia 44: 321 (2020)

Podosphaera ampla Meeboon, S. Takam. \& U. Braun, Mycologia 112 (2): 247 (2020)

Podosphaera pruni-avium Meeboon, S. Takam. \& U. Braun, Mycologia 112 (2): 248 (2020)

Podosphaera pruni-cerasoidis Meeboon, S. Takam. \& U. Braun, Mycologia 112 (2): 250 (2020)

Podosphaera prunigena Meeboon, S. Takam. \& U. Braun, Mycologia 112 (2): 252 (2020) Podosphaera pruni-japonicae Meeboon, S. Takam. \& U. Braun, Mycologia 112 (2): 253 (2020)

Podosphaera pruni-lusitanicae Meeboon, S. Takam. \& U. Braun, Mycologia 112 (2): 254 (2020)

Podosphaera prunina Meeboon, S. Takam. \& U. Braun, Mycologia 112 (2): 255 (2020)

Podosphaera thermopsidicola S. Yadav, S.K. Verma \& Raghv. Singh, Phytotaxa 453 (2): 110 (2020)

*Podosphaera yulii S.Y. Liu \& P.L. Qiu, Fungal Diversity 10.1007/s13225-02000461-7: 51 (2020)

*Polycephalomyces elaphomyceticola W.Y. Chuang, H.A. Ariyaw., J.I. Yang \& Stadler, Mycol. Progr. 19 (1): 102 (2020)

Polycoccum parmotrematis Van den Boom, Acta bot. hung. 62 (3-4): 422 (2020)

Polyporus culmicola C. Sharp \& Ryvarden, Syn. Fung. 40: 110 (2020)

Polyporus gabonensis Decock \& Ryvarden, Syn. Fung. 42: 11 (2020) 
Polyporus laetiporoides Vlasák \& Ryvarden, Syn. Fung. 42: 32 (2020)

Polyscytalum pini-canariensis Crous, Persoonia 45: 315 (2020)

Polyscytalum pinicola Crous, Fungal Systematics and Evolution 6: 214 (2020)

Porina collina Orange, Palice \& Klepsland, Lichenologist 52: 272 (2020)

Porina florensii Diederich \& Ertz, Plant and Fungal Systematics 65 (1): 53 (2020)

*Porodaedalea mongolica Y.D. Wu \& Y. Yuan, Mycosystema 39 (2): 259 (2020)

Porpidia seakensis Fryday, Lichenologist 52 (2): 116 (2020)

Porpidinia brevispora L.S. Yakovchenko \& E.A. Davydov, Phytotaxa 459 (1): 76 (2020) Preussia octosymmetrica Chalange, Bull. Soc. mycol. Fr. 133 (3-4): 251 (2020)

Preussia procaviae Crous, Persoonia 45: 321 (2020)

Proliferodiscus ingens S. Bien \& Damm, MycoKeys 63: 146 (2020)

Pronectria etayoi E. Zimm. \& F. Berger, Herzogia 33: 480 (2020)

Protographum luttrellii Le Renard, Upchurch, Stockey \& Berbee, Mycologia 112 (3): 511 (2020)

Protoparmeliopsis ertzii Bungartz \& Elix, Phytotaxa 431 (1): 72 (2020)

Psammina filamentosa Kolk \& Earl.-Benn., Lichenologist 52 (5): 337 (2020)

Psathyrella atlantica V. Coimbra \& Wartchow, Acta Bot. Brasilica 34 (2): 395 (2020)

Psathyrella cystoindica Voto, Boll. Assoc. Micol. Ecol. Romana 109 (1): 13 (2020)

Psathyrella tierramayorae Voto, Boll. Assoc. Micol. Ecol. Romana 109 (1): 13 (2020)

*Pseudoasteromassaria aquatica W. Dong, $\mathrm{H}$. Zhang \& K.D. Hyde, Fungal Diversity 10.1007/s13225-020-00463-5: 93 (2020)

*Pseudoastrosphaeriella aquatica W. Dong, H. Zhang \& K.D. Hyde, Fungal Diversity 10.1007/s13225-020-00463-5: 168 (2020)
*Pseudobactrodesmium aquaticum W. Dong, H. Zhang \& K.D. Hyde, Frontiers in Microbiology 11 (no. 565): 5 (2020)

*Pseudobactrodesmium chiangmaiensis X.D. Yu, W. Dong \& K.D. Hyde, Frontiers in Microbiology 11 (no. 565): 5 (2020)

*Pseudobensingtonia fusiformis Q.M. Wang, F.Y. Bai \& A.H. Li, Stud. Mycol. 96: 109 (2020)

*Pseudocamarosporium camporesii Q. Tian \& K.D. Hyde, Fungal Diversity 100: 54 (2020)

Pseudocapulatispora clematidis Phukhams. \& K.D. Hyde, Fungal Diversity 10.1007/s13225-020-00448-4: 47 (2020)

Pseudocapulatispora longiappendiculata Mapook \& K.D. Hyde, Fungal Diversity 101: 48 (2020)

Pseudocercospora diospyri-japonicae U. Braun, Fungal Systematics and Evolution 6: 101 (2020)

Pseudocercospora diospyriphila H.D. Shin \& U. Braun, Fungal Systematics and Evolution 6: 103 (2020)

Pseudocercospora ershadii M. Bakhshi, U. Braun \& Zare, Fungal Systematics and Evolution 6: 105 (2020)

Pseudocercospora hamiltoniani Raghvendra Singh, Sanjeet Kumar Verma, Sanjay Yadav, Puja Bhojak \& Shambhu Kumar, Phytotaxa 458 (4): 283 (2020)

Pseudocercospora kakiicola C. Nakash., Fungal Systematics and Evolution 6: 113 (2020)

Pseudocercospora kobayashiana C. Nakash., Fungal Systematics and Evolution 6: 115 (2020)

*Pseudocercospora maetaengensis J.F. Li \& R. Phookamsak, Fungal Diversity 100: 16 (2020)

Pseudocercospora pseudomusae Crous \& Carlier, Fungal Systematics and Evolution 7: 15 (2020) 
Pseudocercospora seropedicensis Andrade, Medeiros \& Inácio, Mycotaxon 135 (1): 121 (2020)

Pseudocercospora solani-cernui Rembinski, Oliveira \& Inácio, Mycotaxon 135 (1): 124 (2020)

Pseudocercospora tessellata U. Braun, Fungal Systematics and Evolution 6: 117 (2020)

Pseudocercospora xiancao R. Kirschner, Pl. Dis. 104 (9): 2393 (2020)

*Pseudochaetosphaeronema kunmingense D.P. Wei, Wanas. \& K.D. Hyde, Fungal Diversity 100: 75 (2020)

Pseudocoleophoma clematidis Phukhams. \& K.D. Hyde, Fungal Diversity 10.1007/s13225-020-00448-4: 21 (2020)

*Pseudocoleophoma rusci W.J. Li, Camporesi \& K.D. Hyde, Fungal Diversity 100: 676 (2020)

*Pseudoconlarium punctiforme N.G. Liu, K.D. Hyde \& J.K. Liu, Fungal Diversity 100: 191 (2020)

Pseudocosmospora hypoxylicola Lechat \& J. Fourn., Ascomycete.org 12 (2): 6 (2020)

*Pseudodactylaria camporesiana W. Dong, Doilom \& K.D. Hyde, Fungal Diversity 100: 217 (2020)

*Pseudogymnoascus guizhouensis Zhi Y. Zhang, Y.F. Han \& Z.Q. Liang, Frontiers in Microbiology 11 (no. 572596): 5 (2020)

Pseudogymnoascus palmeri A.E. Rea-Ireland, Smyth, D.L. Lindner \& Overton, Persoonia 45: 355 (2020)

*Pseudogymnoascus shaanxiensis $\mathrm{Zhi}$ Y. Zhang, Y.F. Han \& Z.Q. Liang, Frontiers in Microbiology 11 (no. 572596): 9 (2020)

*Pseudogymnoascus sinensis Zhi Y. Zhang, Y.F. Han \& Z.Q. Liang, Frontiers in Microbiology 11 (no. 572596): 11 (2020)

*Pseudohydnum brunneiceps Y.L. Chen, M.S. Su \& L.P. Zhang, Phytotaxa 441 (1): 91 (2020)

*Pseudohyphozyma hydrangeae Q.M. Wang,
F.Y. Bai \& A.H. Li, Stud. Mycol. 96: 130 (2020)

*Pseudohyphozyma lulangensis Q.M. Wang, F.Y. Bai \& A.H. Li, Stud. Mycol. 96: 130 (2020)

*Pseudoleucosporidium fasciculatum Babeva \& Lisichk. ex M. Groenew. \& Q.M. Wang, Stud. Mycol. 96: 136 (2020)

Pseudolophiostoma chiangraiense Phukhams. \& K.D. Hyde, Fungal Diversity 10.1007/s13225-020-00448-4: 49 (2020)

Pseudolophiostoma clematidis Phukhams. \& K.D. Hyde, Fungal Diversity 10.1007/s13225-020-00448-4: 51 (2020)

Pseudomarasmius efibulatus R.H. Petersen, Mycotaxon 135: 31 (2020)

Pseudomarasmius obscurus R.H. Petersen, Mycotaxon 135: 50 (2020)

Pseudomarasmius patagonianus R.H. Petersen, Mycotaxon 135: 68 (2020)

Pseudomarasmius quercophylloides R.H. Petersen, Mycotaxon 135: 73 (2020)

Pseudomassarina clematidis Phukhams, Camporesi \& K.D. Hyde, Fungal Diversity 10.1007/s13225-020-00448-4: 101 (2020)

*Pseudomicrothyrium thailandicum X.Y. Zeng, Hongsanan \& K.D. Hyde, Fungal Diversity 10.1007/s13225-020-00462-6: 79 (2020)

Pseudomurilentithecium camporesii Mapook \& K.D. Hyde, Fungal Diversity 100: 69 (2020)

Pseudomurilentithecium clematidis Brahmanage, Camporesi \& K.D. Hyde, Mycosphere 11 (1): 2492 (2020)

Pseudoomphalina anticostica Lebeuf, Kennedy \& I. Saar, Botany 98 (2): 95 (2020) Pseudoophiobolus pseudoitalicus Senan. \& K.D. Hyde, Asian Journal of Mycology 3 (1): 121 (2020)

Pseudopalawania siamensis Mapook \& K.D. Hyde, Biomolecules 10 (no. 569): 12 (2020) Pseudopaucispora hyalinospora Samarak. \& 
K.D. Hyde, Mycosphere 11: 2502 (2020)

Pseudopenidiella vietnamensis IturrietaGonzález, Gené \& Dania García, Microorganisms 8 (4, no. 48): 8 (2020)

*Pseudopestalotiopsis avicenniae Norphanphoun, T.C. Wen \& K.D. Hyde, Mycosphere 10 (1): 558 (2020)

*Pseudopestalotiopsis curvatispora Norphanphoun, T.C. Wen \& K.D. Hyde, Mycosphere 10 (1): 558 (2020)

*Pseudopestalotiopsis rhizophorae Norphanphoun, T.C. Wen \& K.D. Hyde, Mycosphere 10 (1): 560 (2020)

Pseudopestalotiopsis thailandica

Norphanphoun \& K.D. Hyde, Mycosphere 10 (1): 562 (2020)

Pseudopezicula betulae Crous, Persoonia 44: 341 (2020)

Pseudophaeomoniella globosa C.F.J. Spies, Carlucci, Moyo, van Jaarsveld, Halleen \& L. Mostert, Persoonia 45: 206 (2020)

*Pseudoplectania sinica Qiao Zhang \& Jie Zhang, Mycosystema 39 (8): 1478 (2020)

Pseudopyricularia javanii A. Pordel \& G. Ghorbani, Persoonia 45: 381 (2020)

*Pseudorobillarda parasiamensis $\mathrm{N}$. Tangthirasunun, W.J. Li \& K.D. Hyde, Fungal Diversity 100: 693 (2020)

Pseudoroussoella chromolaenae Mapook \& K.D. Hyde, Fungal Diversity 101: 88 (2020) *Pseudoscopulariopsis asperispora Z.F. Zhang \& L. Cai, Fungal Diversity 10.1007/s13225-020-00453-7: 88 (2020)

Pseudosperma amabile Bandini, B. Oertel \& Wehr, Czech Mycol. 72 (2): 232 (2020)

Pseudosperma amoris Bandini \& B. Oertel, Czech Mycol. 72 (2): 236 (2020)

Pseudosperma brunneoumbonatum Saba \& Khalid, MycoKeys 69: 11 (2020)

*Pseudosperma citrinostipes Y.G. Fan \& W.J. Yu, Phytotaxa 450 (1): 10 (2020)

Pseudosperma conviviale Cervini, Bizio \& $\mathrm{P}$. Alvarado, Index Fungorum 441: 1 (2020)
Pseudosperma flavorimosum Jabeen \& Khalid, Mycotaxon 135 (1): 187 (2020)

Pseudosperma melleum Cervini, Bizio \& P. Alvarado, Index Fungorum 441: 1 (2020)

Pseudosperma napaeanum Bandini \& B. Oertel, Czech Mycol. 72 (2): 241 (2020)

Pseudosperma pinophilum Saba \& Khalid, MycoKeys 69: 18 (2020)

Pseudosperma ponderosum Cervini, Bizio \& P. Alvarado, Index Fungorum 441: 2 (2020)

Pseudosperma salentinum Cervini, Bizio \& P. Alvarado, Index Fungorum 441: 1 (2020)

Pseudosperma triaciculare Saba \& Khalid, MycoKeys 69: 20 (2020)

Pseudostaurosphaeria chromolaenae Mapook \& K.D. Hyde, Fungal Diversity 101: 81 (2020)

Pseudostaurosphaeria chromolaenicola Mapook \& K.D. Hyde, Fungal Diversity 101: 82 (2020)

*Pseudosterigmatospora motuoensis Q.M. Wang, F.Y. Bai \& A.H. Li, Stud. Mycol. 96: 111 (2020)

Pseudosubramaniomyces septatus TorresGarcia, Gené \& Dania García, Persoonia 45: 383 (2020)

Pseudotetraploa rajmachiensis Rajeshkumar, K.D. Hyde \& Wijayaw., Fungal Diversity 100: 116 (2020)

Pseudothyridariella chromolaenae Mapook \& K.D. Hyde, Fungal Diversity 101: 98 (2020)

Pseudotremella lacticolor Satoh \& Makimura ex Yurkov, Stud. Mycol. 96: 136 (2020)

Pseudotricharina lanigera Healy, D. Torres, Pfister \& M.E. Sm., Ascomycete.org 12 (2): 34 (2020)

Pseudotrichia ambigua A. Bell \& D.P. Mahoney, Ascomycete.org 12 (6): 216 (2020)

Pseudotruncatella camporesii Goonas. \& Hyde, Fungal Diversity 100: 221 (2020)

*Pseudoxylomyces aquaticus W. Dong, H. Zhang \& K.D. Hyde, Fungal Diversity 
10.1007/s13225-020-00463-5: 119 (2020)

Pseudozeugandromyces tachypori De Kesel \& Haelew., Sydowia 72: 291 (2020)

*Psoroma capense Elvebakk, S.G. Hong \& Rämä, Lichenologist 52 (5): 346 (2020)

Psoroma esterhuyseniae Elvebakk, Lichenologist 52 (5): 348 (2020)

Pterygiopsis densisidiata Aptroot, Oliveira Junior \& M. Cáceres, Bryologist 123 (4): $628(2020)$

Puccinia dimorphothecae-cuneatae Berndt, Mycol. Progr. 19 (4): 248 (2020)

Puccinia feliciicola Berndt, Mycol. Progr. 19 (4): 249 (2020)

*Puccinia xinyuanensis J.G. Song, Aime \& B. Xu, Phytotaxa 459 (1): 88 (2020)

*Purpureofaciens aquatica W. Dong, H. Zhang \& K.D. Hyde, Fungal Diversity 10.1007/s13225-020-00463-5: 57 (2020)

Purpureomyces maesotensis Luangsa-ard, Noisrip. Thanakitp. \& Samson, Stud. Mycol. 95: 242 (2020)

Purpureomyces pyriformis Luangsa-ard, Noisrip., Himaman, Mongkols. \& Thanakitp. \& Samson, Stud. Mycol. 95: 242 (2020)

Pusillomyces spinulosus César, Bandala \& Montoya, Mycol. Progr. 19 (10): 1024 (2020)

*Pygmaeomyces pinuum E. Walsh \& N. Zhang, Mycologia 10.1080/00275514.2020.1803649: 8 (2020)

*Pygmaeomyces thomasii E. Walsh \& N. Zhang, Mycologia 10.1080/00275514.2020.1803649: 8 (2020)

Pyrenochaetopsis chromolaenae Mapook \& K.D. Hyde, Fungal Diversity 101: 87 (2020) Pyrenochaetopsis rajhradensis Spetik, Eichmeier \& Berraf-Tebbal, Persoonia 45: 385 (2020)

Pyrenodesmia vernadskii S.Y. Kondr., T.O. Kondr. \& Parnikoza, Acta bot. hung. 62 (34): 257 (2020)

Pyrenula muriciliata Diederich \& Ertz, Plant and Fungal Systematics 65 (1): 57 (2020)
Pyrenula rubroacutispora Aptroot, Archive For Lichenology 17: 8 (2020)

*Pyrrhulomyces amariceps E.J. Tian \& Matheny, Mycologia 10.1080/00275514.2020.1816067: 13 (2020)

Pythium iranense Badali, Abrinbana \& Abdollahz., Cryptog. Mycol. 41 (11): 185 (2020)

Pythium monoclinum Abrinbana, Abdollahz. \& Badali, Cryptog. Mycol. 41 (11): 185 (2020)

*Quaeritorhiza haematococci Longcore, D.R. Simmons, T.Y. James \& S. Qin, Mycologia 112 (3): 611 (2020)

Raffaelea borbonica M. Procter, M.J. Wingf. \& Marinc., Fungal Systematics and Evolution 6: 309 (2020)

Ramalina krogiae Guissard \& Sérus., MycoKeys 73: 36 (2020)

Ramaria atractospora Franchi \& M. Marchetti, Index Fungorum 467: 4 (2020)

Ramaria brunneolilacina Franchi \& M. Marchetti, Index Fungorum 467: 4 (2020)

Ramaria gracilioides Franchi \& M. Marchetti, Index Fungorum 467: 5 (2020)

Ramaria ichnusensis Franchi, A. Gennari \& M. Marchetti, Index Fungorum 467: 5 (2020)

Ramaria kafaensis Gminder \& Christan, Z. Mykol. 86 (2): 222 (2020)

Ramaria luteoaurantiaca Franchi \& M. Marchetti, Index Fungorum 433: 1 (2020)

Ramaria ossolana Franchi \& M. Marchetti, Index Fungorum 467: 5 (2020)

Ramaria paraconcolor Franchi \& M. Marchetti, Index Fungorum 467: 6 (2020)

Ramaria thindii K. Das, Hembrom, A. Parihar \& A. Ghosh, Cryptog. Mycol. 41 (4): 88 (2020)

Ramariopsis hirtipes Franchi \& M. Marchetti, Index Fungorum 467: 7 (2020)

Ramiphialis ronuroensis F.R. Barbosa, Fiúza \& R.F. Castañeda, Mycotaxon 135 (2): 294 (2020) 
Ramonia xylophila Aptroot, Archive For Lichenology 17: 9 (2020)

Ramusculicola clematidis Phukhams. \& K.D. Hyde, Fungal Diversity 10.1007/s13225020-00448-4: 127 (2020)

Ravenelia doidgeae $\mathrm{M}$. Ebinghaus, Begerow \& W. Maier, Mycol. Progr. 19 (4): 272 (2020)

Ravenelia dumeti $\mathrm{M}$. Ebinghaus, W. Maier \& Begerow, Mycol. Progr. 19 (4): 273 (2020)

Ravenelia modjadji M. Ebinghaus, W. Maier \& Begerow, Mycol. Progr. 19 (4): 273 (2020)

Ravenelia molopa M. Ebinghaus, W. Maier \& Begerow, Mycol. Progr. 19 (4): 271 (2020)

Ravenelia moloto $\mathrm{W}$. Maier, M. Ebinghaus \& Begerow, Mycol. Progr. 19 (4): 266 (2020)

Ravenelia spinifera W. Maier, M. Ebinghaus \& Begerow, Mycol. Progr. 19 (4): 270 (2020)

Redingeria uniseptata Kalb, Archive For Lichenology 18: 9 (2020)

Redonographa parvispora R. Miranda, Barcenas-Peña \& Lücking, Bryologist 123 (2): 134 (2020)

Rehmanniella poeltiana S.Y. Kondr. \& Hur, Acta bot. hung. 62 (3-4): 267 (2020)

*Remotididymella brunnea L.W. Hou, L. Cai \& Crous, Stud. Mycol. 96: 336 (2020)

*Remotididymella humicola L.W. Hou, L. Cai \& Crous, Stud. Mycol. 96: 338 (2020)

Resupinatus odoratus C.K. Pradeep, C. Bijeesh \& A.M. Kumar, Phytotaxa 464 (2): 170 (2020)

*Retiboletus ater Yan C. Li \& T. Bau, MycoKeys 67: 37 (2020)

* Retiboletus sinogriseus Yan C. Li \& T. Bau, MycoKeys 67: 40 (2020)

Rhachomyces latellae W. Rossi \& M. Leonardi, Cryptog. Mycol. 41 (1): 4 (2020)

Rhachomyces spinosus Santam. \& CuestaSegura, Nova Hedwigia 110 (3-4): 362 (2020)

Rhachomyces spiralis W. Rossi \& M. Leonardi, Cryptog. Mycol. 41 (1): 4 (2020)

Rhachomyces toledanoi W. Rossi \& M.
Leonardi, Cryptog. Mycol. 41 (1): 4 (2020)

Rhagadodidymellopsis endocarpi Fern.-Brime, Gaya, Llimona \& Nav.-Ros., Plant and Fungal Systematics 65: 177 (2020)

*Rhamphoriopsis sympodialis C.G. Lin, K.D. Hyde \& Jian K. Liu, Fungal Diversity 10.1007/s13225-020-00458-2: 31 (2020)

Rhexocercosporidium senecionis Phutthacharoen \& K.D. Hyde, Fungal Diversity 100: 155 (2020)

Rhizocarpon austroalpinum P.M. McCarthy, Elix \& Kantvilas, Australas. Lichenol. 86: 37 (2020)

Rhizocarpon exiguum P.M. McCarthy, Elix \& Kantvilas, Australas. Lichenol. 86: 39 (2020)

Rhizocarpon haidense Brodo \& Fryday, Lichenologist 52 (2): 116 (2020)

Rhizocarpon torquatum P.M. McCarthy, J.A. Elix \& G. Kantvilas, Australas. Lichenol. 86: 44 (2020)

Rhizophydium jobii Hassett, Mycologia 112 (4): 788 (2020)

Rhodocybe dominicana T.J. Baroni \& Angelini, Mycol. Progr. 19 (10): 1091 (2020) Rhodocybe fusipes Silva-Filho, D.L. Komura $\&$ Wartchow, Sydowia 72: 164 (2020)

Rhodocybe pseudoalutacea T.J. Baroni, Lodge \& Lacey, Mycol. Progr. 19 (10): 1092 (2020)

*Rhodofomitopsis pseudofeei B.K. Cui \& Shun Liu, Fungal Diversity 10.1007/s13225020-00461-7: 138 (2020)

Rhodophana griseobrunnea E. Musumeci, Errotari 17: 227 (2020)

*Rhodophana guandishanensis L. Fan \& C. Yang, Phytotaxa 455 (2): 176 (2020)

*Rhodosporidiobolus fuzhouensis Q.M. Wang, F.Y. Bai \& A.H. Li, Stud. Mycol. 96: 124 (2020)

*Rhodosporidiobolus jianfalingensis Q.M. Wang, F.Y. Bai \& A.H. Li, Stud. Mycol. 96: 123 (2020)

*Rhodosporidiobolus platycladi Q.M. Wang, F.Y. Bai \& A.H. Li, Stud. Mycol. 96: 123 
(2020)

*Rhomboidia wuliangshanensis C.L. Zhao, Mycotaxon 134 (4): 656 (2020)

* Rhynchogastrema nanyangensis F.L. Hui \& Q.H. Niu ex Xin Zhan Liu, F.Y. Bai, M. Groenew., Boekhout \& Yurkov, Stud. Mycol. 96: 136 (2020)

Rhytidhysteron bruguierae Dayarathne, Mycosphere 11 (1): 20 (2020)

Rhytidhysteron camporesii Ekanayaka \& K.D. Hyde, Fungal Diversity 100: 18 (2020)

Rhytidhysteron chromolaenae Mapook \& K.D. Hyde, Fungal Diversity 101: 11 (2020)

Rhytidhysteron erioi Ekanayaka \& K.D. Hyde, Fungal Diversity 100: 21 (2020)

Rhytidhysteron magnoliae N.I. de Silva \& K.D. Hyde, Asian Journal of Mycology 3 (1): 298 (2020)

Rhytidhysteron neohysterinum CobosVillagran, Hern.-Rodr., R. Valenz. \& Raymundo, Acta Bot. Mexicana 127: e1675, 3 (2020)

*Rigidoporus juniperinus Gafforov, L.W. Zhou \& E. Langer, Fungal Diversity 10.1007/s13225-020-00461-7: 135 (2020)

Rigidoporus subvinctus Ryvarden, Syn. Fung. 40: 105 (2020)

Rigidoporus subvinctus Ryvarden, Syn. Fung. 42: 29 (2020)

Rimaconus multiguttulatus Dayarathne \& K.D. Hyde, Mycosphere 11 (1): 126 (2020)

Rinodina austroisidiata Elix \& Kantvilas, Australas. Lichenol. 87: 82 (2020)

Rinodina fineranii Elix, C. Edler \& $\mathrm{H}$. Mayrhofer, Australas. Lichenol. 86: 95 (2020)

Rinodina gerhardii H. Mayrhofer \& Elix, Australas. Lichenol. 87: 73 (2020)

Rinodina heronensis H. Mayrhofer \& Elix, Australas. Lichenol. 87: 74 (2020)

Rinodina klauskalbii H. Mayrhofer \& Elix, Australas. Lichenol. 87: 75 (2020)

Rinodina lecideopsis Sheard \& C.A. Morse,
Bryologist 123 (1): 31 (2020)

Rinodina malcolmii Elix, C. Edler \& $\mathrm{H}$. Mayrhofer, Australas. Lichenol. 86: 96 (2020)

Rinodina subcolobina Van den Boom, Acta bot. hung. 62 (3-4): 425 (2020)

*Robertozyma ningxiaensis Q.M. Wang, F.Y. Bai \& A.H. Li, Stud. Mycol. 96: 117 (2020)

Robillarda sohagensis Abdel-Wahab, AbulEzz \& Bakhit, Sydowia 72: 293 (2020)

Roridomyces pseudoirritans A.A. Kiyashko, Persoonia 44: 443 (2020)

* Rosettozyma cystopteridis Q.M. Wang \& F.Y. Bai, Stud. Mycol. 96: 119 (2020)

*Rosettozyma motuoensis Q.M. Wang \& F.Y. Bai, Stud. Mycol. 96: 119 (2020)

*Rosettozyma petaloides Q.M. Wang, F.Y. Bai \& A.H. Li, Stud. Mycol. 96: 119 (2020)

*Roussoella aquatica W. Dong, H. Zhang \& K.D. Hyde, Fungal Diversity 10.1007/s13225-020-00463-5: 172 (2020)

Roussoella arundinacea Crous \& R.K. Schumach., Fungal Systematics and Evolution 6: 217 (2020)

Roussoella margidorensis E. Bovio, Prigione, A. Poli \& Varese, Diversity 12 (4, no. 144): 12 (2020)

Roussoella mediterranea A. Poli, E. Bovio, Prigione \& Varese, Diversity 12 (4, no. 144): 13 (2020)

Roussoella padinae Prigione, E. Bovio, A. Poli \& Varese, Diversity 12 (4, no. 144): 14 (2020)

Rufoplaca aesanensis S.Y. Kondr. \& Hur, Acta bot. hung. 62 (3-4): 272 (2020)

*Ruinenia bangxiensis Q.M. Wang, F.Y. Bai \& A.H. Li, Stud. Mycol. 96: 109 (2020)

*Ruinenia diospyri Nakase, Tsuzuki, F.L. Lee, Jindam. \& M. Takash. ex Q.M. Wang, F.Y. Bai, M. Groenew. \& Boekhout, Stud. Mycol. 96: 137 (2020)

*Ruinenia fanjingshanensis Q.M. Wang, F.Y. Bai \& A.H. Li, Stud. Mycol. 96: 109 (2020) 
*Ruinenia lunata Q.M. Wang, F.Y. Bai \& A.H. Li, Stud. Mycol. 96: 110 (2020)

*Ruinenia pyrrosiae Nakase, Tsuzuki, F.L. Lee, Jindam. \& M. Takash. ex Q.M. Wang, F.Y. Bai, M. Groenew. \& Boekhout, Stud. Mycol. 96: 137 (2020)

Rusavskia drevlyanica S.Y. Kondr. \& O.O. Orlov, Acta bot. hung. 62 (1-2): 88 (2020)

Rusavskia indochinensis S.Y. Kondr., G.K. Mishra, S. Nayaka \& D.K. Upreti, Acta bot. hung. 62 (1-2): 94 (2020)

Russula antsikana Buyck \& Randrianjohany, Cryptog. Mycol. 41 (4): 92 (2020)

Russula ashihoi K. Das, A. Ghosh, Buyck \& Hembrom, Nordic Jl Bot. 38 (11): 3 (2020)

Russula baniyakundensis A. Ghosh, K. Das \& D. Chakr., Phytotaxa 483 (3): 249 (2020)

Russula benghalensis S. Paloi \& K. Acharya, Fungal Diversity 10.1007/s13225-02000461-7: 146 (2020)

*Russula byssina G.J. Li \& C.Y. Deng, Mycosystema 39 (4): 626 (2020)

*Russula cremicolor G.J. Li \& C.Y. Deng, Mycosystema 39 (4): 628 (2020)

*Russula glutinosoides Buyck \& X.H. Wang, Mycosphere 11 (1): 296 (2020)

Russula indonigra K. Das, A. Ghosh, Buyck \& Hembrom, Nordic Jl Bot. 38 (11): 7 (2020)

*Russula latolamellata Y. Song \& L.H. Qiu, Cryptog. Mycol. 41 (14): 222 (2020)

*Russula nigrocarpa S.Y. Zhou, Y. Song \& L.H. Qiu, Cryptog. Mycol. 41 (14): 224 (2020)

*Russula ochrobrunnea S.Y. Zhou, Y. Song \& L.H. Qiu, Cryptog. Mycol. 41 (14): 232 (2020)

Russula orientipurpurea Wisitr., H. Lee \& Y.W. Lim, MycoKeys 75: 15 (2020)

*Russula pseudocrustosa G.J. Li \& C.Y. Deng, Mycosystema 39 (9): 1670 (2020)

Russula rubricolor Jabeen, Naseer \& Khalid, Mycotaxon 135 (4): 769 (2020)

Russula shanglaensis S. Ullah, Khalid \& Fiaz,
Turkish Journal of Botany 44 (1): 87 (2020)

Russula shawarensis Kiran \& Khalid, Persoonia 45: 387 (2020)

*Russula subpunctipes J. Song, Phytotaxa 459 (1): 19 (2020)

Sagiolechia phaeospora Fryday \& T. Sprib., Lichenologist 52 (2): 119 (2020)

*Saitozyma paraflava Golubev \& J.P. Samp. ex Xin Zhan Liu, F.Y. Bai, M. Groenew. \& Boekhout, Stud. Mycol. 96: 137 (2020)

*Saitozyma pseudoflava Q.M. Wang, F.Y. Bai \& A.H. Li, Stud. Mycol. 96: 87 (2020)

Sajamaea mycophila Flakus, Piątek \& Rodr. Flakus, Mycol. Progr. 19 (1): 6 (2020)

*Sakaguchia melibiophila M. Groenew., Q.M. Wang \& F.Y. Bai, Stud. Mycol. 96: 114 (2020)

Salsuginea rhizophorae Dayarathne, E.B.G. Jones \& K.D. Hyde, Mycosphere 11 (1): 67 (2020)

*Samsoniella alpina H. Yu, Y.B. Wang, Y. Wang \& Zhu L. Yang, Fungal Diversity 103: 24 (2020)

*Samsoniella antleroides H. Yu, Y.B. Wang, Y. Wang, Q. Fan \& Zhu L. Yang, Fungal Diversity 103: 25 (2020)

*Samsoniella cardinalis H. Yu, Y.B. Wang, Y. Wang, Q. Fan \& Zhu L. Yang, Fungal Diversity 103: 27 (2020)

*Samsoniella coleopterorum W.H. Chen, Y.F. Han \& Z.Q. Liang, MycoKeys 74: 9 (2020)

*Samsoniella cristata H. Yu, Y.B. Wang, Y. Wang, Q. Fan \& Zhu L. Yang, Fungal Diversity 103: 27 (2020)

*Samsoniella hymenopterorum W.H. Chen, Y.F. Han \& Z.Q. Liang, MycoKeys 74: 10 (2020)

*Samsoniella kunmingensis $\mathrm{H}$. Yu, Y.B. Wang, Y. Wang, Q. Fan \& Zhu L. Yang, Fungal Diversity 103: 32 (2020)

*Samsoniella lanmaoa H. Yu, Y.B. Wang, Y. Wang, Q. Fan \& Zhu L. Yang, Fungal Diversity 103: 32 (2020) 
*Samsoniella lepidopterorum W.H. Chen, Y.F. Han \& Z.Q. Liang, MycoKeys 74: 11 (2020) *Samsoniella ramosa $\mathrm{H}$. Yu, Y.B. Wang, Y. Wang, Q. Fan \& Zhu L. Yang, Fungal Diversity 103: 35 (2020)

*Samsoniella tortricidae H. Yu, Y.B. Wang, Y. Wang, Q. Fan \& Zhu L. Yang, Fungal Diversity 103: 36 (2020)

*Samsoniella yunnanensis H. Yu, Y.B. Wang, Y. Wang, D.E. Duan \& Zhu L. Yang, Fungal Diversity 103: 38 (2020)

*Sanguinoderma flavovirens Y.F. Sun \& B.K. Cui, Persoonia 44: 227 (2020)

*Sanguinoderma laceratum Y.F. Sun \& B.K. Cui, Persoonia 44: 228 (2020)

*Sanguinoderma microporum Y.F. Sun \& B.K. Cui, Persoonia 44: 230 (2020)

*Sanguinoderma reniforme Y.F. Sun \& B.K. Cui, Persoonia 44: 231 (2020)

*Sanguinoderma sinuosum Y.F. Sun \& B.K. Cui, Persoonia 44: 235 (2020)

*Sarcodon coactus Y.H. Mu \& H.S. Yuan, MycoKeys 66: 89 (2020)

*Sarcodon grosselepidotus Y.H. Mu \& H.S. Yuan, MycoKeys 66: 93 (2020)

*Sarcodon lidongensis Y.H. Mu \& H.S. Yuan, MycoKeys 66: 96 (2020)

*Sarcogyne alcesensis K. Knudsen, J.N. Adams, Kocourk. \& Y. Wang, Bryologist 123 (1): 18 (2020)

*Sarcogyne bernardinensis K. Knudsen, J.N. Adams, Kocourk. \& Y. Wang, Bryologist 123 (1): 18 (2020)

*Sarcogyne convexa K. Knudsen, J.N. Adams, Kocourk. \& Y. Wang, Bryologist 123 (1): 23 (2020)

Sarcogyne molongloensis P.M. McCarthy \& Elix, Australas. Lichenol. 86: 74 (2020)

Sarcogyne paradoxa Kocourk. \& K. Knudsen, Mycotaxon 135 (1): 458 (2020)

Sarcogyne porphyricola P.M. McCarthy \& Elix, Australas. Lichenol. 86: 76 (2020)

Sarcogyne terrulenta P.M. McCarthy \& Elix,
Australas. Lichenol. 86: 78 (2020)

*Sarcogyne wheeleri K. Knudsen, J.N. Adams, Kocourk. \& Y. Wang, Bryologist 123 (1): 25 (2020)

Sarcopodium durantae R.H. Perera, E.B.G. Jones \& K.D. Hyde, Mycosphere 11 (1): 2161 (2020)

Sarocladium clematidis Phukhams., Ertz, Gerstmans \& K.D. Hyde, Mycosphere 10.1007/s13225-020-00448-4: 183 (2020)

Sarocladium graminicola R.M. Anjos, S.I. Moreira, S.S. Costa \& P.G. Cardoso, Mycol. Progr. 19 (6): 610 (2020)

*Sarocladium sparsum J.H. Ou, G.C. Lin \& C.Y. Chen, Mycol. Progr. 19 (1): 75 (2020)

*Sarocladium spirale J.H. Ou, G.C. Lin \& C.Y. Chen, Mycol. Progr. 19 (1): 76 (2020)

Satchmopsis metrosideri Crous, Persoonia 44: 345 (2020)

Satchmopsis pini Crous, Persoonia 44: 347 (2020)

Savitreea pentosicarens Sakpuntoon, Angchuan, Boonmak, Khunnamw., N. Jacques, Grondin, Casareg. \& Srisuk, Int. J. Syst. Evol. Microbiol. 70 (11): 5669 (2020)

Saxiloba hawaiiensis Lücking, Moncada \& Flynn, Plant and Fungal Systematics 65 (2): 582 (2020)

* Scheffersomyces anoplophorae C.Y. Chai \& F.L. Hui, MycoKeys 71: 95 (2020)

*Scheffersomyces jinghongensis C.Y. Chai \& F.L. Hui, MycoKeys 71: 92 (2020)

*Scheffersomyces paraergatensis C.Y. Chai \& F.L. Hui, MycoKeys 71: 93 (2020)

Sclerenchymomyces clematidis Phukhams. \& K.D. Hyde, Fungal Diversity 10.1007/s13225-020-00448-4: 41 (2020)

Sclerococcum fissurinae Pérez-Ort., Lichenologist 52 (2): 120 (2020)

Scleroderma nastii Raut, Stud. Fung. 5 (1): 53 (2020)

*Scleroramularia vermispora G.Y. Sun \& L. Gao, Fungal Diversity 10.1007/s13225-020- 
00461-7: 47 (2020)

Scolecoxyphium blechni Abdollahz. \& Crous, Stud. Mycol. 95: 409 (2020)

Scolecoxyphium blechnicola Abdollahz. \& Crous, Stud. Mycol. 95: 409 (2020)

Scolecoxyphium leucadendri Abdollahz. \& Crous, Stud. Mycol. 95: 409 (2020)

Scolecoxyphium phylicae Abdollahz. \& Crous, Stud. Mycol. 95: 410 (2020)

Scoliciosporum fabisporum Fryday \& I. Medeiros, S. Afr. J. Bot. 132: 475 (2020)

Scorias aphidis Abdollahz. \& Crous, Stud. Mycol. 95: 410 (2020)

Scorias camelliae Abdollahz. \& Crous, Stud. Mycol. 95: 410 (2020)

Scytinopogon minisporus J. AlvarezManjarrez, M. Villegas \& R. Garibay-Orijel, Fungal Diversity 10.1007/s13225-02000461-7: 241 (2020)

*Scytinostroma yunnanense C.L. Zhao, Phytotaxa 451 (2): 150 (2020)

Septoglomus mediterraneum A. Guillén, F.J. Serrano-Tamay, J.B. Peris \& I. Arrillaga, Mycologia 112 (4): 823 (2020)

Septoriella camporesii Goonas. \& Hyde, Fungal Diversity 100: 108 (2020)

Serendipita restingae Y. Fritsche, M.A. Selosse \& M.P. Guerra, Mycorrhiza 10.1007/s00572-020-01000-7: 5 (2020)

*Serendipita sacchari L. Xie, Y.Y. Long \& Y.L. Chen, Mycotaxon 135 (3): 582 (2020)

Serendipita whamiae Dearnaley, T.W. May \& Linde, Persoonia 45: 389 (2020)

Serusiauxia inexpectata Ertz \& Diederich, Plant and Fungal Systematics 65 (1): 61 (2020)

*Serusiauxiella filifera S.H. Jiang, Lücking \& J.C. Wei, Fungal Diversity 102: 275 (2020)

*Serusiauxiella flagellata S.H. Jiang, Lücking \& J.C. Wei, Fungal Diversity 102: 277 (2020)

*Serusiauxiella sinensis S.H. Jiang, Lücking \& J.C. Wei, Fungal Diversity 102: 277 (2020) Setoapiospora thailandica Mapook \& K.D.
Hyde, Fungal Diversity 100: 135 (2020)

Setoarthopyrenia chromolaenae Mapook \& K.D. Hyde, Fungal Diversity 101: 92 (2020)

*Setophaeosphaeria microspora Z.F. Zhang \& L. Cai, Fungal Diversity 10.1007/s13225020-00453-7: 47 (2020)

*Shrungabeeja aquatica W. Dong, G.N. Wang \& H. Zhang, Fungal Diversity 10.1007/s13225-020-00463-5: 180 (2020)

*Sidera minutissima Y.C. Dai, F. Wu, G.M. Gates \& Rui Du, MycoKeys 68: 122 (2020)

*Sidera parallela Y.C. Dai, F. Wu, G.M. Gates \& Rui Du, Mycosphere 68: 123 (2020)

*Sidera srilankensis Y.C. Dai, F. Wu, G.M. Gates \& Rui Du, MycoKeys 68: 127 (2020)

*Sidera tenuis Y.C. Dai, F. Wu, G.M. Gates \& Rui Du, MycoKeys 68: 128 (2020)

Sigarispora clematidicola Phukhams., Camporesi \& K.D. Hyde, Fungal Diversity 10.1007/s13225-020-00448-4: 53 (2020)

Sigarispora clematidis Phukhams. \& K.D. Hyde, Fungal Diversity 10.1007/s13225020-00448-4: 53 (2020)

Sigarispora montanae Phukhams., Sue \& K.D. Hyde, Fungal Diversity 10.1007/s13225020-00448-4: 55 (2020)

Similitrichoconis wongii R.F. Castañeda, M. Vera \& D. Sosa, Mycotaxon 135 (4): 833 (2020)

*Simplicillium album Z.F. Zhang \& L. Cai, Fungal Diversity 10.1007/s13225-02000453-7: 77 (2020)

*Simplicillium humicola Z.F. Zhang \& L. Cai, Fungal Diversity 10.1007/s13225-02000453-7: 78 (2020)

*Simplicillium yunnanense H. Yu, Y.B. Wang, Y. Wang \& Zhu L. Yang, Fungal Diversity 103: 40 (2020)

*Skeletocutis subalbomarginata Rui Du \& Y.C. Dai, Mycosystema 39 (4): 642 (2020)

*Slooffia globosa Q.M. Wang, F.Y. Bai \& A.H.

Li, Stud. Mycol. 96: 131 (2020)

*Solicoccozyma gelidoterrea Q.M. Wang, F.Y. 
Bai \& A.H. Li, Stud. Mycol. 96: 99 (2020)

*Solomyces sinensis Zhi Y. Zhang, Y.F. Han \& Z.Q. Liang, Frontiers in Microbiology 11 (no. 572596): 11 (2020)

Sordaria clematidis Phukhams. \& K.D. Hyde, Fungal Diversity 10.1007/s13225-02000448-4: 153 (2020)

*Spathaspora elongata C.Y. Chai \& F.L. Hui, MycoKeys 75: 36 (2020)

*Spathaspora jiuxiensis C.Y. Chai \& F.L. Hui, MycoKeys 75: 40 (2020)

*Spathaspora mengyangensis C.Y. Chai \& F.L. Hui, MycoKeys 75: 38 (2020)

*Spathaspora parajiuxiensis C.Y. Chai \& F.L. Hui, MycoKeys 75: 41 (2020)

*Spathaspora rosae C.Y. Chai \& F.L. Hui, MycoKeys 75: 43 (2020)

Spegazzinia musae Samarakoon, Phookamsak, Wanas., Chomnunti \& K.D. Hyde, MycoKeys 70: 27 (2020)

Spermospora arrhenatheri Crous, Fungal Systematics and Evolution 7: 90 (2020)

Spermospora loliiphila Crous, Fungal Systematics and Evolution 7: 92 (2020)

Spermospora zeae Crous, Fungal Systematics and Evolution 7: 93 (2020)

Sphaeropsis chromolaenicola Mapook \& K.D. Hyde, Fungal Diversity 101: 113 (2020)

Sphaerulina neoaceris Crous \& Bulgakov, Fungal Systematics and Evolution 6: 218 (2020)

Sphaerulina salicicola Crous \& Bulgakov, Fungal Systematics and Evolution 6: 219 (2020)

Spilonema maritimum T. Sprib. \& Fryday, Lichenologist 52 (2): 121 (2020)

*Sporidesmium dulongense Z.L. Luo, K.D. Hyde \& H.Y. Su, Mycosphere 11 (1): 864 (2020)

*Sporobolomyces cellobiolyticus Q.M. Wang, F.Y. Bai \& A.H. Li, Stud. Mycol. 96: 121 (2020)

*Sporobolomyces ellipsoideus Q.M. Wang,
F.Y. Bai \& A.H. Li, Stud. Mycol. 96: 121 (2020)

*Sporobolomyces primogenomicus Q.M. Wang \& F.Y. Bai, Stud. Mycol. 96: 123 (2020)

*Sporobolomyces reniformis Q.M. Wang, F.Y. Bai \& A.H. Li, Stud. Mycol. 96: 121 (2020) Sporothrix oleae Musvuugwa, L.L. Dreyer \& F. Roets, Mycol. Progr. 19 (1): 87 (2020)

Squamulea chelonia Bungartz \& Søchting, Plant and Fungal Systematics 65 (2): 563 (2020)

Squamulea coreana S.Y. Kondr. \& Hur, Acta bot. hung. 62 (3-4): 274 (2020)

Squamulea humboldtiana Bungartz \& Søchting, Plant and Fungal Systematics 65 (2): 656 (2020)

Squamulea oceanica Bungartz \& Søchting, Plant and Fungal Systematics 65 (2): 566 (2020)

Squamulea osseophila Søchting \& Bungartz, Plant and Fungal Systematics 65 (2): 566 (2020)

Squamulea uttarkashiana S.Y. Kondr., Upreti, Nayak \& A. Thell, Acta bot. hung. 62 (3-4): 376 (2020)

Srinivasanomyces kangrensis S. Rana \& S.K. Singh, Fungal Diversity 100: 159 (2020)

Stagonospora poaceicola Tennakoon, Phookamsak \& K.D Hyde, Index Fungorum 11: 2505 (2020)

*Stagonosporopsis cucumeris L.W. Hou, L. Cai \& Crous, Stud. Mycol. 96: 324 (2020)

*Stagonosporopsis sambucella L.W. Hou, L. Cai \& Crous, Stud. Mycol. 96: 326 (2020)

*Stagonosporopsis stuijvenbergii Hern.-Restr., L.W. Hou, L. Cai \& Crous, MycoKeys 65: 82 (2020)

*Stagonosporopsis weymaniae Hern.-Restr., L.W. Hou, L. Cai \& Crous, MycoKeys 65: 84 (2020)

Starmera foglemanii J.D. Moreira, A.R.O. Santos, Lachance \& C.A Rosa, Int. J. Syst. 
Evol. Microbiol. 10.1099/ijsem.0.004300: 4 (2020)

Starmera ilhagrandensis C.G. Morais, A.R.O. Santos, Lachance \& C.A. Rosa, Int. J. Syst. Evol. Microbiol. 10.1099/ijsem.0.004300: 5 (2020)

Starmerella vitis Čadež, Lachance, DrumondeNeves, Sipiczki \& G. Péter, Antonie van Leeuwenhoek 113: 1295 (2020)

Stemonitis pseudoflavogenita A. Vlasenko \& Novozh., Phytotaxa 447 (2): 139 (2020)

Stemphylium artemisiae Brahmanage, Camporesi \& K.D. Hyde, Mycosphere 11: 2526 (2020)

Stemphylium carpobroti Crous, Persoonia 45: 307 (2020)

Stemphylium clematidis Wanas., Camporesi \& K.D. Hyde, Fungal Diversity 10.1007/s13225-020-00448-4: 91 (2020)

Stereum ellipticum Ryvarden, Syn. Fung. 40: 52 (2020)

*Sterigmatospora layueensis Q.M. Wang, F.Y. Bai \& A.H. Li, Stud. Mycol. 96: 111 (2020)

*Sterila eucalypti Crous, M. Shen \& Y. Zhang ter, Stud. Mycol. 96: 212 (2020)

Sticta acyphellata Moncada \& Lücking, Plant and Fungal Systematics 65 (1): 106 (2020)

Sticta antoniana Moncada \& Lücking, Plant and Fungal Systematics 65 (1): 108 (2020)

Sticta borinquensis Merc.-Díaz \& Lücking, Taxon 69 (5): 865 (2020)

Sticta corymbosa Merc.-Díaz \& Lücking, Taxon 69 (5): 867 (2020)

Sticta densiphyllidiata Merc.-Díaz \& Lücking, Taxon 69 (5): 869 (2020)

Sticta emmanueliana Moncada, Lücking \& Lumbsch, Plant and Fungal Systematics 65 (1): 109 (2020)

Sticta flynnii Moncada \& Lücking, Plant and Fungal Systematics 65 (1): 110 (2020)

Sticta guilartensis Merc.-Díaz \& Lücking, Taxon 69 (5): 871 (2020)

Sticta harrisii Merc.-Díaz \& Lücking, Taxon
69 (5): 873 (2020)

Sticta hawaiiensis Moncada \& Lücking, Plant and Fungal Systematics 65 (1): 111 (2020)

Sticta parvilobata Merc.-Díaz \& Lücking, Taxon 69 (5): 875 (2020)

Sticta riparia Merc.-Díaz \& Lücking, Taxon 69 (5): 877 (2020)

Sticta smithii Moncada \& Lücking, Plant and Fungal Systematics 65 (1): 113 (2020)

Sticta tainorum Merc.-Díaz \& Lücking, Taxon 69 (5): 879 (2020)

Sticta waikamoi Moncada \& Lücking, Plant and Fungal Systematics 65 (1): 114 (2020)

Stigmatomyces ferrugineus W. Rossi \& M. Leonardi, Cryptog. Mycol. 41 (1): 6 (2020)

Stigmidium anguinellicola Etayo \& Aptroot, Bryologist 123 (3): 488 (2020)

Stigmidium fellhanerae Van den Boom, Ascomycete.org 12 (5): 202 (2020)

Stigmidium pyrenulae Van den Boom, Acta bot. hung. 62 (3-4): 427 (2020)

Stilbohypoxylon elaeidis Konta \& K.D. Hyde, Mycosphere 11 (1): 2642 (2020)

Stouffera gilkeyae D.G. Knapp, Zagyva, Trappe \& Kovács, Mycologia 112 (4): 814 (2020)

Striatosphaeria castanea Réblová \& J. Fourn., MycoKeys 74: 40 (2020)

Strigula depressa J.J. Woo, Lücking \& Hur, Phytotaxa 443 (1): 4 (2020)

Strigula multiformis J.J. Woo, Lücking \& Hur, Phytotaxa 443 (1): 8 (2020)

Strigula pyrenuloides Aptroot, Archive For Lichenology 20: 4 (2020)

Strongwellsea acerosa Eilenberg \& Humber, J. Invert. Path. 175 (no. 107444): 3 (2020)

Strongwellsea tigrinae Eilenberg \& Humber, J. Invert. Path. 175 (no. 107444): 3 (2020)

*Stylonectria qilianshanensis Z.Q. Zeng \& W.Y. Zhuang, MycoKeys 71: 126 (2020)

*Submersispora variabilis W. Dong, H. Zhang \& K.D. Hyde, Fungal Diversity 10.1007/s13225-020-00463-5: 122 (2020) 
Subplenodomus meldolanus Brahmanage, Camporesi \& K.D. Hyde, Mycosphere 11: 2499 (2020)

Subplenodomus urticae D. Pem, Camporesi, Jeewon \& K.D. Hyde, Frontiers in Microbiology 11 (no. 1303): 9 (2020)

Suhomyces drosophilae R. Jadhav, S. Tiwari, R. Avchar, M. Groenew. \& A. Baghela, Int. J. Syst. Evol. Microbiol. 10.1099/ijsem.0.004358: 4 (2020)

Suttonomyces cephalophylli Crous, Persoonia 45: 299 (2020)

*Symmetrospora rhododendri Q.M. Wang, F.Y. Bai \& A.H. Li, Stud. Mycol. 96: 115 (2020)

Symphytocarpus macrosporus A. Vlasenko, Karstenia 58 (2): 395 (2020)

Synarthonia leproidica Ertz, Aptroot \& Diederich, Lichenologist 52: 263 (2020)

Synarthonia xanthosarcographoides Aptroot, Archive For Lichenology 17: 10 (2020)

Syncephalastrum contaminatum A.S. Urquhart \& A. Idnurm, Mycoscience 61: 114 (2020)

Syncephalis latigena R.K. Benj. \& Benny, Mycologia 112 (3): 561 (2020)

Tahromyces munnarensis Hanafy, Lanjekar, Dhakephalkar, T.M. Callaghan, Dagar, G.W. Griff., Elshahed \& N.H. Youssef, Mycologia 112 (6): 1235 (2020)

*Talaromyces albisclerotius B.D. Sun, A.J. Chen, Houbraken \& Samson, MycoKeys 68: 88 (2020)

*Talaromyces aspriconidius B.D. Sun, A.J. Chen, Houbraken \& Samson, MycoKeys 68: 90 (2020)

*Talaromyces brevis B.D. Sun, A.J. Chen, Houbraken \& Samson, MycoKeys 68: 92 (2020)

*Talaromyces guizhouensis B.D. Sun, A.J. Chen, Houbraken \& Samson, MycoKeys 68: 94 (2020)

Talaromyces pulveris Crous, Persoonia 45:
293 (2020)

*Talaromyces rufus B.D. Sun, A.J. Chen, Houbraken \& Samson, MycoKeys 68: 99 (2020)

*Talaromyces tenuis B.D. Sun, A.J. Chen, Houbraken \& Samson, MycoKeys 68: 86 (2020)

*Talaromyces yunnanensis Doilom \& C.F. Liao, Frontiers in Microbiology 11 (no. 585215): 18 (2020)

*Tapellaria parvimuriformis W.C. Wang \& J.C. Wei, Lichenologist 52 (5): 380 (2020)

Telimena trichiliae Mardones \& M. Piepenbr., Mycol. Progr. 19 (12): 1596 (2020)

*Tenuitholiascus porinoides S.H. Jiang \& J.C. Wei, IMA Fungus 11 (no. 1): 11 (2020)

Termitomyces acriumbonatus Usman \& Khalid, Phytotaxa 477 (2): 221 (2020)

*Termitomyces floccosus S.M. Tang, Raspé \& S.H. Li, Phytotaxa 439 (3): 234 (2020)

Termitomyces sheikhupurensis Izhar, Khalid \& H. Bashir, Turkish Journal of Botany 44: 696 (2020)

*Termitomyces upsilocystidiatus S.M. Tang, Raspé \& K.D. Hyde, Phytotaxa 439 (3): 236 (2020)

*Terrestriporia alba Y.C. Dai, B.K. Cui, F. Wu, Y. Yuan \& Jia J. Chen, Mycosphere 11 (1): 2761 (2020)

*Terriera guizhouensis C.L. Hou \& S.R. Cai, Mycol. Progr. 19 (9): 828 (2020)

*Terriera houjiazhuangensis C.L. Hou \& S.R. Cai, Mycol. Progr. 19 (9): 831 (2020)

*Terriera ilicis C.L. Hou \& S.R. Cai, Mycol. Progr. 19 (9): 832 (2020)

Tetramelas flindersianus Elix, Australas. Lichenol. 87: 10 (2020)

Tetramelas gariwerdensis Elix, Australas. Lichenol. 87: 11 (2020)

Tetramelas oreophilus Elix \& Kantvilas, Australas. Lichenol. 87: 22 (2020)

Tetraploa dwibahubeeja Rajeshkumar, K.D. Hyde \& S. Lad, Fungal Diversity 100: 117 
(2020)

Tetraploa pseudoaristata Rajeshkumar, K.D. Hyde \& G. Anand, Fungal Diversity 100: 119 (2020)

*Tetraploa puzheheiensis W. Dong, H. Yang \& H. Zhang, Fungal Diversity 10.1007/s13225-020-00463-5: 183 (2020)

Tetraploa thrayabahubeeja Rajeshkumar, K.D. Hyde \& G. Anand, Fungal Diversity 100: $120(2020)$

*Tetraploa yunnanensis W. Dong, H. Yang \& H. Zhang, Fungal Diversity 10.1007/s13225-020-00463-5: 184 (2020)

Tetrapyrgos brevicystidiata D.L. Komura, J.S. Oliveira \& Moncalvo, Phytotaxa 440 (3): 199 (2020)

Tetrapyrgos cerebrata D.L. Komura, J.S. Oliveira \& Moncalvo, Phytotaxa 440 (3): 201 (2020)

Tetrapyrgos crassicystidiata D.L. Komura, J.S. Oliveira \& Moncalvo, Phytotaxa 440 (3): 203 (2020)

Tetrapyrgos griseibrunnea D.L. Komura, J.S. Oliveira \& Moncalvo, Phytotaxa 440 (3): 205 (2020)

Tetrapyrgos novinigripes D.L. Komura, J.S. Oliveira \& Moncalvo, Phytotaxa 440 (3): 207 (2020)

Tetrapyrgos similinigripes D.L. Komura, J.S. Oliveira \& Moncalvo, Phytotaxa 440 (3): 209 (2020)

*Teunia betulae K. Sylvester, Q.M. Wang \& Hittinger ex Q.M. Wang, F.Y. Bai \& A.H. Li, Stud. Mycol. 96: 86 (2020)

*Teunia globosa Q.M. Wang, F.Y. Bai \& A.H. Li, Stud. Mycol. 96: 87 (2020)

*Teunia helanensis Q.M. Wang, F.Y. Bai \& A.H. Li, Stud. Mycol. 96: 86 (2020)

*Teunia korlaensis Q.M. Wang, F.Y. Bai \& A.H. Li, Stud. Mycol. 96: 87 (2020)

Teunia siamensis Khunnamw. \& Limtong, Int. J. Syst. Evol. Microbiol. 10.1099/ijsem.0.004219: 5 (2020)
*Teunia tronadorensis V. de García, Zalar, Brizzio, Gunde-Cim. \& van Broock ex Q.M. Wang, F.Y. Bai \& A.H. Li, Stud. Mycol. 96: 86 (2020)

Thalloloma intermedium Kalb, Archive For Lichenology 18: 10 (2020)

Thaxteriellopsis obliqua M. Niranjan \& V.V. Sarma, Fungal Diversity 10.1007/s13225020-00461-7: 43 (2020)

Theleporus africanus Decock \& Ryvarden, Syn. Fung. 42: 12 (2020)

Thelidium carbonaceum P.M. McCarthy \& Kantvilas, Australas. Lichenol. 86: 109 (2020)

Thelocarpon immersum Fryday, Lichenologist 52 (2): 124 (2020)

Thelopsis spinulosa Aptroot, Archive For Lichenology 20: 5 (2020)

*Thozetella bambusicola H.B. Jiang, Phookamsak \& K.D. Hyde, Fungal Diversity 10.1007/s13225-020-00458-2: 21 (2020)

Thyrostroma alhagi D. Pem, Gafforov \& K.D. Hyde, Fungal Diversity 10.1007/s13225020-00458-2: 6 (2020)

Thysanorea cantrelliae Hern.-Restr., R. van Doorn \& Crous, Fungal Systematics and Evolution 6: 18 (2020)

Thysanorea seifertii Hern.-Restr., R. van Doorn \& Crous, Fungal Systematics and Evolution 6: 18 (2020)

Thysanorea yunnanensis Hern.-Restr. \& Crous, Fungal Systematics and Evolution 6: 21 (2020)

*Tingoldiago clavata D.F. Bao, L. Xu \& H.Y. Su, MycoKeys 65: 128 (2020)

*Tingoldiago hydei D.F. Bao, Z.L. Luo \& H.Y. Su, MycoKeys 65: 126 (2020)

Toensbergia blastidiata T. Sprib. \& Tønsberg, Lichenologist 52 (2): 125 (2020)

Tolypocladium flavonigrum Noisrip., Tasan., Khons. \& Luangsa-ard, Persoonia 45: 393 (2020)

*Tomentella asiae-orientalis H.S. Yuan, X. Lu 
\& Y.C. Dai, Fungal Diversity 10.1007/s13225-020-00461-7: 152 (2020)

*Tomentella atrobadia H.S. Yuan \& Y.C. Dai, Fungal Diversity 10.1007/s13225-02000461-7: 153 (2020)

*Tomentella atrocastanea H.S. Yuan \& Y.C. Dai, Fungal Diversity 10.1007/s13225-02000461-7: 154 (2020)

*Tomentella aureomarginata H.S. Yuan \& Y.C. Dai, Fungal Diversity 10.1007/s13225020-00461-7: 156 (2020)

*Tomentella brevis H.S. Yuan \& Y.C. Dai, Fungal Diversity 10.1007/s13225-02000461-7: 156 (2020)

*Tomentella brunneoflava H.S. Yuan \& Y.C. Dai, Fungal Diversity 10.1007/s13225-02000461-7: 158 (2020)

*Tomentella brunneogrisea H.S. Yuan \& Y.C. Dai, Fungal Diversity 10.1007/s13225-02000461-7: 159 (2020)

*Tomentella capitatocystidiata H.S. Yuan, X. $\mathrm{Lu} \&$ Y.C. Dai, Fungal Diversity 10.1007/s13225-020-00461-7: 161 (2020)

*Tomentella changbaiensis H.S. Yuan, X. Lu \& Y.C. Dai, Fungal Diversity 10.1007/s13225-020-00461-7: 163 (2020)

*Tomentella citrinocystidiata H.S. Yuan \& Y.C. Dai, Fungal Diversity 10.1007/s13225020-00461-7: 163 (2020)

*Tomentella coffeae H.S. Yuan \& Y.C. Dai, Fungal Diversity 10.1007/s13225-02000461-7: 165 (2020)

*Tomentella conclusa H.S. Yuan, X. Lu \& Y.C. Dai, Fungal Diversity 10.1007/s13225020-00461-7: 166 (2020)

*Tomentella cystidiata H.S. Yuan \& Y.C. Dai, Fungal Diversity 10.1007/s13225-02000461-7: 167 (2020)

*Tomentella dimidiata H.S. Yuan, X. Lu \& Y.C. Dai, Fungal Diversity 10.1007/s13225020-00461-7: 169 (2020)

*Tomentella duplexa H.S. Yuan, X. Lu \& Y.C. Dai, Fungal Diversity 10.1007/s13225-020-
00461-7: 171 (2020)

*Tomentella efibulata H.S. Yuan \& Y.C. Dai, Fungal Diversity 10.1007/s13225-02000461-7: 171 (2020)

*Tomentella efibulis H.S. Yuan, X. Lu \& Y.C. Dai, Fungal Diversity 10.1007/s13225-02000461-7: 173 (2020)

*Tomentella farinosa H.S. Yuan \& Y.C. Dai, Fungal Diversity 10.1007/s13225-02000461-7: 175 (2020)

*Tomentella flavidobadia H.S. Yuan \& Y.C. Dai, Fungal Diversity 10.1007/s13225-02000461-7: 177 (2020)

*Tomentella fuscocrustosa H.S. Yuan, X. Lu \& Y.C. Dai, Fungal Diversity 10.1007/s13225-020-00461-7: 181 (2020)

*Tomentella fuscofarinosa H.S. Yuan, X. Lu \& Y.C. Dai, Fungal Diversity 10.1007/s13225-020-00461-7: 181 (2020)

*Tomentella fuscogranulosa H.S. Yuan \& Y.C. Dai, Fungal Diversity 10.1007/s13225020-00461-7: 183 (2020)

*Tomentella fuscopelliculosa H.S. Yuan, X. $\mathrm{Lu} \&$ Y.C. Dai, Fungal Diversity 10.1007/s13225-020-00461-7: 185 (2020)

*Tomentella globospora H.S. Yuan \& Y.C. Dai, Fungal Diversity 10.1007/s13225-02000461-7: 187 (2020)

*Tomentella gloeocystidiata H.S. Yuan \& Y.C. Dai, Fungal Diversity 10.1007/s13225-02000461-7: 187 (2020)

*Tomentella griseocastanea H.S. Yuan, X. Lu \& Y.C. Dai, Fungal Diversity 10.1007/s13225-020-00461-7: 191 (2020)

*Tomentella griseofusca H.S. Yuan \& Y.C. Dai, Fungal Diversity 10.1007/s13225-02000461-7: 193 (2020)

*Tomentella griseomarginata H.S. Yuan, X. $\mathrm{Lu} \&$ Y.C. Dai, Fungal Diversity 10.1007/s13225-020-00461-7: 195 (2020)

*Tomentella inconspicua H.S. Yuan \& Y.C. Dai, Fungal Diversity 10.1007/s13225-02000461-7: 199 (2020) 
*Tomentella incrustata H.S. Yuan, X. Lu \& Y.C. Dai, Fungal Diversity 10.1007/s13225020-00461-7: 201 (2020)

*Tomentella interrupta H.S. Yuan \& Y.C. Dai, Fungal Diversity 10.1007/s13225-02000461-7: 203 (2020)

*Tomentella liaoningensis H.S. Yuan \& Y.C. Dai, Fungal Diversity 10.1007/s13225-02000461-7: 206 (2020)

*Tomentella longechinulata H.S. Yuan, X. Lu \& Y.C. Dai, Fungal Diversity 10.1007/s13225-020-00461-7: 211 (2020)

*Tomentella longiaculeifera H.S. Yuan \& Y.C. Dai, Fungal Diversity 10.1007/s13225-02000461-7: 209 (2020)

*Tomentella megaspora H.S. Yuan, X. Lu \& Y.C. Dai, Fungal Diversity 10.1007/s13225020-00461-7: 213 (2020)

*Tomentella olivacea H.S. Yuan \& Y.C. Dai, Fungal Diversity 10.1007/s13225-02000461-7: 215 (2020)

*Tomentella olivaceobrunnea H.S. Yuan, X. $\mathrm{Lu} \&$ Y.C. Dai, Fungal Diversity 10.1007/s13225-020-00461-7: 217 (2020)

*Tomentella pallidobrunnea H.S. Yuan, X. Lu \& Y.C. Dai, Fungal Diversity 10.1007/s13225-020-00461-7: 221 (2020)

*Tomentella pallidomarginata H.S. Yuan, X. Lu \& Y.C. Dai, Fungal Diversity 10.1007/s13225-020-00461-7: 223 (2020)

*Tomentella parvispora H.S. Yuan \& Y.C. Dai, Fungal Diversity 10.1007/s13225-02000461-7: 225 (2020)

*Tomentella pertenuis H.S. Yuan \& Y.C. Dai, Fungal Diversity 10.1007/s13225-02000461-7: 227 (2020)

*Tomentella qingyuanensis H.S. Yuan \& Y.C. Dai, Fungal Diversity 10.1007/s13225-02000461-7: 229 (2020)

*Tomentella segregata H.S. Yuan, Xu Lu \& Y.C. Dai, Fungal Diversity 10.1007/s13225020-00461-7: 233 (2020)

*Tomentella separata H.S. Yuan, X. Lu \& Y.C.
Dai, Fungal Diversity 10.1007/s13225-02000461-7: 235 (2020)

*Tomentella stipitata H.S. Yuan, X. Lu \& Y.C. Dai, Fungal Diversity 10.1007/s13225-02000461-7: 236 (2020)

*Tomentella storea H.S. Yuan \& Y.C. Dai, Fungal Diversity 10.1007/s13225-02000461-7: 237 (2020)

*Torula camporesii Phookamsak, E.F. Yang \& K.D. Hyde, Fungal Diversity 100: 123 (2020)

*Torula hydei J.F. Li, Phookamsak \& Jeewon, PLoS ONE 15 (2): e0228067, 8 (2020)

*Torula thailandica N.G. Liu, Jian K. Liu \& K.D. Hyde, Mycosphere 11 (1): 2045 (2020)

Trametes lamelloporus Ryvarden, Syn. Fung. 42: 92 (2020)

Trametes parvispora Olou, Yorou \& Langer, MycoKeys 65: 38 (2020)

Trapelia atrocarpa Elix \& P.M. McCarthy, Australas. Lichenol. 86: 102 (2020)

Trapelia kosciuszkoensis Elix, Australas. Lichenol. 86: 103 (2020)

Trapelia occidentalis Elix, Australas. Lichenol. 87: 40 (2020)

Trapelia pruinosa Elix \& P.M. McCarthy, Australas. Lichenol. 86: 105 (2020)

Trapelia rosettiformis Elix \& P.M. McCarthy, Australas. Lichenol. 87: 41 (2020)

Trapelia terrestris Elix \& P.M. McCarthy, Australas. Lichenol. 87: 43 (2020)

Trechispora copiosa Meiras-Ottoni \& Gibertoni, Mycologia 20 (2): 210 (2020)

Trechispora hondurensis Schoutteten \& Haelew., Sydowia 72: 296 (2020)

Trechispora mollis Chikowski \& K.H. Larss., Mycol. Progr. 19 (12): 1406 (2020)

Trechispora torrendii Chikowski \& K.H. Larss., Mycol. Progr. 19 (12): 1408 (2020)

Tremateia camporesii Samarak. \& K.D. Hyde, Fungal Diversity 100: 56 (2020)

Tremateia chiangraiensis Mapook \& K.D. Hyde, Fungal Diversity 101: 38 (2020)

Tremateia chromolaenae Mapook \& K.D. 
Hyde, Fungal Diversity 101: 40 (2020)

Tremateia lamiacearum Samarak. \& K.D. Hyde, Fungal Diversity 100: 57 (2020)

Tremateia thailandensis Mapook \& K.D. Hyde, Fungal Diversity 101: 44 (2020)

Trematosphaeria magenta A. Alidadi, Mycol. Progr. 19 (12): 1578 (2020)

*Tremella basidiomaticola Xin Zhan Liu \& F.Y. Bai, Stud. Mycol. 96: 137 (2020)

Tremella imshaugiae Diederich, Coppins, R.C. Harris, Millanes \& Wedin, Bull. Soc. Nat. luxemb. 122: 242 (2020)

*Tremella shuangheensis Q.M. Wang, F.Y. Bai \& A.H. Li, Stud. Mycol. 96: 84 (2020)

Tremella tubulosae Diederich, Coppins, J.C. Zamora, Millanes \& Wedin, Bull. Soc. Nat. luxemb. 122: 243 (2020)

Triadelphia hexaformispora Chuaseehar., Somrith. \& Boonyuen, Mycol. Progr. 19 (2): 134 (2020)

*Trichoderma arenarium F. Cai, M.Y. Ding \& I. S. Druzhinina, Journal of Applied Microbiology 10.1111/jam.14751: 9 (2020)

Trichoderma azevedoi M.C. Valadares-Inglis \& P.W. Inglis, PLoS ONE 15 (3): e0228485, $12(2020)$

*Trichoderma ceratophylli Z.F. Yu \& X. Du, Fungal Diversity 10.1007/s13225-02000461-7: 85 (2020)

*Trichoderma dorothopsis A.A. Tomah \& J.Z. Zhang, Biological Control 145 (no. 104261): 6 (2020)

*Trichoderma gansuanum Z.Q. Zeng \& W.Y. Zhuang, MycoKeys 71: 127 (2020)

*Trichoderma lentinulae Jing Z. Sun \& X.Z. Liu, MycoKeys 73: 116 (2020)

Trichoderma peberdyi M.C. Valadares-Inglis \& P.W. Inglis, PLoS ONE 15 (3): e0228485, 12 (2020)

*Trichoderma vermifimicola Jing Z. Sun \& X.Z. Liu, MycoKeys 73: 118 (2020)

*Trichoderma xixiacum Jing Z. Sun \& X.Z. Liu, MycoKeys 73: 120 (2020)
*Trichoderma zelobreve Jing Z. Sun \& X.Z. Liu, MycoKeys 73: 121 (2020)

Tricholoma kenanii I. Acar, S. Aldemir \& A. Dizkirici, Sydowia 72: 302 (2020)

*Tricholoma orienticolossum X. Xu, Y.Y. Cui \& Zhu L. Yang, Phytotaxa 443 (2): 159 (2020)

*Tricholoma orientifulvum X. Xu, Y.Y. Cui \& Zhu L. Yang, Phytotaxa 443 (2): 161 (2020)

Tricholomopsis alborufescens Larue, Bull. Soc. mycol. Fr. 133 (1-2): 179 (2020)

*Tricholomopsis rubroaurantiaca Hosen \& T.H. Li, Mycoscience 61 (6): 343 (2020)

*Tricholyophyllum brunneum Qing Cai, G. Kost \& Zhu L. Yang, Mycosystema 39 (9): 1734 (2020)

Trichomerium camporesii Marasinghe \& K.D. Hyde, Asian Journal of Mycology 3 (1): 179 (2020)

*Trichomerium cicatricatum L. Su, W. Sun \& M.C. Xiang, Journal of Fungi 6 (4, no. 187): 21 (2020)

*Trichomerium flexuosum W. Sun, X.Z. Liu \& M.C. Xiang, Journal of Fungi 6 (4, no. 187): 23 (2020)

*Trichomerium lapideum L. Su, W. Sun \& M.C. Xiang, Journal of Fungi 6 (4, no. 187): 24 (2020)

*Trichomerium leigongense W. Sun, L. Su \& M.C. Xiang, Journal of Fungi 6 (4, no. 187): 25 (2020)

Trichomerium syzygii Crous, Fungal Systematics and Evolution 6: 224 (2020)

Trichophoma cylindrospora Magaña-Dueñas, Cano \& Stchigel, Persoonia 44: 445 (2020)

Trichophyton africanum Čmoková \& Hubka, Fungal Diversity 104: 369 (2020)

Trichophyton europaeum Čmoková \& Hubka, Fungal Diversity 104: 360 (2020)

Trichophyton indotineae R. Kano, U. Kimura, M. Kakurai, J. Hiruma, H. Kamata, Y. Suga \& K. Harada, Mycopathologia 10.1007/s11046-020-00455-8: 11 (2020) 
Trichophyton japonicum Čmoková \& Hubka, Fungal Diversity 104: 362 (2020)

Trimmatostroma commonii Diederich, Zhurb. $\&$ van den Boom, Folia cryptog. Estonica 57: $34(2020)$

*Trimorphomyces sakaeraticus Fungsin, M. Takash. \& Nakase ex Xin Zhan Liu, F.Y. Bai, M. Groenew. \& Boekhout, Stud. Mycol. 96: 137 (2020)

Triseptata sexualis Boonmee \& Phookamsak, Phytotaxa 447 (4): 257 (2020)

Tritirachium batistae J.D.P. Bezerra \& C.M. Souza-Motta, Acta Bot. Brasilica 34 (2): 296 (2020)

Troglomyces twitteri Santam., Enghoff \& Reboleira, MycoKeys 67: 48 (2020)

Tropicoporus texanus A.A. Brown, D.P. Lawr. \& K. Baumgartner, Pl. Path. 69 (2): 216 (2020)

Trypethelium aureornatum Aptroot, Archive For Lichenology 17: 11 (2020)

Trypethelium endoflavum Aptroot, Archive For Lichenology 17: 12 (2020)

Trypethelium xanthostiolornatum Aptroot, Archive For Lichenology 17: 13 (2020)

*Tubakia koreana H.Y. Yun, Mycotaxon 135 (1): 225 (2020)

Tuber alcaracense Ant. Rodr. \& Morte, Persoonia 44: 447 (2020)

Tuber buendiae Ant. Rodr. \& Morte, Persoonia 44: 449 (2020)

Tuber iranicum F. Puliga, M. Illice, M. Iotti, D. Baldo \& A. Zambonelli, Mycologia 112 (5): 935 (2020)

Tuber luomae Trappe, Eberhart, Piña Páez \& Bonit, Fungal Systematics and Evolution 6: 300 (2020)

Tuber lusitanicum Ant. Rodr. \& MuñozMohedano, Persoonia 45: 395 (2020)

*Tubulicrinis xantha C.L. Zhao, Mycoscience 61 (4): 186 (2020)

*Tubulicrinis yunnanensis C.L. Zhao, Mycoscience 61 (4): 187 (2020)
Tubulinosema suzukii S. Biganski, J.T. Wennmann, C.R. Vossbrinck, R. Kaur, J.A. Jehle, R.G. Kleespies, J. Invert. Path. 174 (no. 107440): 10 (2020)

Tulasnella brigadeiroensis E.F.S. Freitas, Meir. Silva \& M.C.M. Kasuya, Scientific Reports 10 (no. 7069): 2 (2020)

Tulasnella hadrolaeliae E.F.S. Freitas, Meir. Silva \& M.C.M. Kasuya, Scientific Reports 10 (no. 7069): 4 (2020)

Tulasnella orchidis E.S. Cruz, E.F.S. Freitas, Meir. Silva \& M.C.M. Kasuya, Scientific Reports 10 (no. 7069): 5 (2020)

Tulasnella zygopetali E.S. Cruz, E.F.S. Freitas, Meir. Silva \& M.C.M. Kasuya, Scientific Reports 10 (no. 7069): 6 (2020)

Tylocliostomum viridifarinosum van den Boom \& Magain, Plant and Fungal Systematics 65 (1): 173 (2020)

Tylopilus hayatae Rodríguez-Ramírez \& Luna-Vega, Phytotaxa 441 (1): 37 (2020)

Tylopilus subotsuensis T.H.G. Pham, A.V. Alexandrova \& O.V. Morozova, Persoonia 45: 397 (2020)

*Tyrannosorus lichenicola Crous, M. Shen \&Y. Zhang ter, Stud. Mycol. 96: 224 (2020) Tyrannosorus pini-sylvestris Crous \& R.K. Schumach., Stud. Mycol. 96: 228 (2020)

Tyromyces chlamydosporus Oba, Mossebo \& Ryvarden, Syn. Fung. 42: 21 (2020)

Tyromyces microsporus Decock \& Ryvarden, Syn. Fung. 42: 12 (2020)

*Tyromyces minutulus Y.C. Dai \& C.L. Zhao, Fungal Diversity 10.1007/s13225-02000461-7: 142 (2020)

Umbelopsis heterosporus C.A. de Souza, D.X. Lima, A.L. Santiago, Fungal Diversity 10.1007/s13225-020-00461-7: 8 (2020)

Umbilicaria orientalis Davydov, Lichenologist 52 (5): 357 (2020)

Unilacryma bispora J.C. Zamora \& S. Ekman, Persoonia 44: 198 (2020)

Uredo myricae Berndt, Mycol. Progr. 19 (4): 


$$
253 \text { (2020) }
$$

Urocystis cumminsii K.G. Savchenko, Carris \& Castl., Mycotaxon 134 (4): 595 (2020)

Urocystis piptatheri N.S. Afshan, A. Anwar, M. Riaz, Sirajuddin \& Khalid, Phytotaxa 444 (2): 82 (2020)

Uromyces hessii Berndt, Mycol. Progr. 19 (4): 252 (2020)

Uromyces rebeccae Bruckart, M. Abbasi \& Aime, Mycologia 112 (3): 547 (2020)

Usnea arianae P. Clerc, E. Caviró \& A. Gerlach, Plant and Fungal Systematics 65 (2): 290 (2020)

Usnea flabelliformis A. Gerlach \& P. Clerc, Plant and Fungal Systematics 65 (2): 283 (2020)

Usnea rubropallens A. Gerlach \& P. Clerc, Plant and Fungal Systematics 65 (2): 286 (2020)

Usnea stipitata A. Gerlach \& P. Clerc, Plant and Fungal Systematics 65 (2): 295 (2020)

*Usnea sulphuridiscoidea S.Y. Guo \& L.F. Han, Phytotaxa 472 (1): 26 (2020)

Usnea tenuicorticata P. Clerc \& A. Gerlach, Plant and Fungal Systematics 65 (2): 188 (2020)

Uzbekistanica pruni Chaiwan, Wanas., Bulgakov \& K.D. Hyde, Fungal Diversity 100: 88 (2020)

Uzbekistanica vitis-viniferae Crous \& Akulov, Fungal Systematics and Evolution 6: 225 (2020)

Vainionora nugrae Bungartz \& Elix, Phytotaxa 431 (1): 75 (2020)

Vanderaaea ammophilae Crous, Fungal Systematics and Evolution 7: 94 (2020)

*Vanderbylia cinnamomea C.L. Zhao, Mycotaxon 135 (1): 375 (2020)

*Vanderwaltozyma huisunica C.F. Lee \& Chin F. Chang, Antonie van Leeuwenhoek 113 (6): 673 (2020)

*Vanderwaltozyma meishanica C.F. Lee \& Chin F. Chang, Antonie van Leeuwenhoek

$$
113 \text { (6): } 671 \text { (2020) }
$$

*Vanderwaltozyma molinica C.F. Lee \& Chin F. Chang, Antonie van Leeuwenhoek 113 (6): 674 (2020)

*Vandijckomycella joseae Hern.-Restr., L.W. Hou, L. Cai \& Crous, MycoKeys 65: 86 (2020)

*Vandijckomycella snoekiae Hern.-Restr., L.W. Hou, L. Cai \& Crous, MycoKeys 65: 88 (2020)

Vanrija meifongana C.F. Lee ex Kachalkin, Yurkov \& Boekhout, Stud. Mycol. 96: 137 (2020)

Vanrija nantouana C.F. Lee ex Kachalkin, Yurkov \& Boekhout, Stud. Mycol. 96: 137 (2020)

Vanrija thermophila Vogelmann, S. Chaves \& C. Hertel ex Kachalkin, Yurkov \& Boekhout, Stud. Mycol. 96: 137 (2020)

*Varioseptispora chinensis L. Qiu, Jian Ma, R.F. Castañeda \& X.G. Zhang, Mycotaxon 135 (4): 754 (2020)

Veloboletus limbatus Fechner \& Halling, Persoonia 45: 399 (2020)

*Venturia albae Crous, M. Shen \&Y. Zhang ter, Stud. Mycol. 96: 230 (2020)

*Venturia australiana Crous, M. Shen \&Y. Zhang ter, Stud. Mycol. 96: 238 (2020)

*Venturia caesiae Crous, M. Shen \&Y. Zhang ter, Stud. Mycol. 96: 243 (2020)

*Venturia finlandica Crous, M. Shen \&Y. Zhang ter, Stud. Mycol. 96: 259 (2020)

Venturia paralias G.C. Hunter, I. Zeil-Rolfe, M. Jourdan \& L. Morin, Persoonia 44: 451 (2020)

*Venturia quebecensis Crous, M. Shen \&Y. Zhang ter, Stud. Mycol. 96: 277 (2020)

Vermiculariopsiella eucalyptigena Crous, Fungal Systematics and Evolution 6: 226 (2020)

Vermilacinia breviloba Spjut \& Sérus., MycoKeys 73: 41 (2020)

Vermilacinia lacunosa Spjut \& Sérus., 
MycoKeys 73: 43 (2020)

Vermilacinia pustulata Spjut \& Sérus., MycoKeys 73: 46 (2020)

Vermilacinia reticulata Spjut \& Sérus., MycoKeys 73: 48 (2020)

Verrucaria bifurcata Pykälä, Kantelinen \& Myllys, MycoKeys 72: 54 (2020)

Verrucaria cavernarum Pykälä \& Myllys, MycoKeys 72: 57 (2020)

Verrucaria difficilis Pykälä \& Myllys, MycoKeys 72: 60 (2020)

Verrucaria fuscozonata Pykälä, Kantelinen \& Myllys, MycoKeys 72: 63 (2020)

Verrucaria kowenensis P.M. McCarthy, Australas. Lichenol. 86: 3 (2020)

Verrucaria kuusamoensis Pykälä, Kantelinen \& Myllys, MycoKeys 72: 65 (2020)

Verrucaria pallidomurina Orange, Phytotaxa 459 (1): 11 (2020)

Verrucaria subdevergens Pykälä \& Myllys, MycoKeys 72: 67 (2020)

Verrucaria tephromela Wahlenb. ex Orange, Phytotaxa 459 (1): 12 (2020)

Verrucaria vacillans Pykälä \& Myllys, MycoKeys 72: 72 (2020)

Verrucocladosporium carpobroti Crous, Persoonia 45: 305 (2020)

Verruconis mangrovei Devadatha V.V. Sarma \& E.B.G. Jones, Fungal Diversity 100: 145 (2020)

Verruconis thailandica Giraldo López \& Crous, Fungal Systematics and Evolution 6: 21 (2020)

Vinositunica ingens Koh. Yamam., Degawa \& A. Yamada, Mycologia 112 (2): 322 (2020)

Vinositunica radiata Koh. Yamam., Degawa \& A. Yamada, Mycologia 112 (2): 320 (2020)

Vishniacozyma alagoana C.R. Félix, D.A. Andrade, J.H. Almeida, H.M. Navarro, Fell \& Landell, Int. J. Syst. Evol. Microbiol. 10.1099/ijsem.0.004193: 3 (2020)

*Vishniacozyma europaea Q.M. Wang, F.Y. Bai \& A.H. Li, Stud. Mycol. 96: 85 (2020)
*Vishniacozyma foliicola Q.M. Wang \& F.Y. Bai ex Yurkov, Stud. Mycol. 96: 137 (2020)

*Vishniacozyma heimaeyensis Vishniac ex Xin Zhan Liu, F.Y. Bai, M. Groenew. \& Boekhout, Stud. Mycol. 96: 137 (2020)

*Vishniacozyma melezitolytica Q.M. Wang, F.Y. Bai \& A.H. Li, Stud. Mycol. 96: 84 (2020)

Vishniacozyma phoenicis Kachalkin, A.S. Venzhik \& M.A. Tomashevskaya, Persoonia 44: 453 (2020)

*Vishniacozyma pseudopenaeus Q.M. Wang, F.Y. Bai \& A.H. Li, Stud. Mycol. 96: 84 (2020)

Vishniacozyma psychrotolerans V. de García, Zalar, Brizzio, Gunde-Cim. \& van Broock ex Yurkov, Stud. Mycol. 96: 137 (2020)

*Vishniacozyma taibaiensis Q.M. Wang \& F.Y. Bai ex Yurkov, Stud. Mycol. 96: 138 (2020)

*Vishniacozyma tephrensis Vishniac ex Xin Zhan Liu, F.Y. Bai, M. Groenew. \& Boekhout, Stud. Mycol. 96: 138 (2020)

Volutella delonicis R.H. Perera, E.B.G. Jones \& K.D. Hyde, Mycosphere 11 (1): 2164 (2020)

Volvariella paludosa Kapitonov \& E.F. Malysheva, Persoonia 44: 455 (2020)

Volvariella turcica O. Kaygusuz \& $\mathrm{H}$. Knudsen, Mycologia 112 (3): 580 (2020)

Vredendaliella oleae C.F.J. Spies, Moyo, Halleen \& L. Mostert, Persoonia 45: 206 (2020)

*Wardomycopsis dolichi Z.F. Zhang \& L. Cai, Fungal Diversity 10.1007/s13225-02000453-7: 91 (2020)

*Wardomycopsis ellipsoconidiophora Z.F. Zhang \& L. Cai, Fungal Diversity 10.1007/s13225-020-00453-7: 91 (2020)

*Wardomycopsis fusca Z.F. Zhang, F. Liu \& L. Cai, Fungal Diversity 10.1007/s13225-02000453-7: 91 (2020)

*Westerdykella aquatica H.Y. Song \& D.M. 
Hu, Mycotaxon 135 (2): 287 (2020)

*Wickerhamiella osmotolerans Sakpuntoon, Angchuan, Boonmak, Chin F. Chang, S.M. Liu, C.F. Lee, Limtong \& Srisuk, Int. J. Syst. Evol. Microbiol. 10.1099/ijsem.0.004075: 3 (2020)

*Wickerhamiella tropicalis Sakpuntoon, Angchuan, Boonmak, Chin F. Chang, S.M. Liu, C.F. Lee, Limtong \& Srisuk, Int. J. Syst. Evol. Microbiol. 10.1099/ijsem.0.004075: 5 (2020)

Wickerhamiella verensis Belloch, A.I. Pelaez, Menendez-Vega, J. Sanchez \& Kurtzman, Int. J. Syst. Evol. Microbiol. 70 (4): 2424 (2020)

Wicklowia phuketensis M.S. Calabon, S. Boonmee, E.B.G Jones \& K.D. Hyde, Phytotaxa 542 (1): 61 (2020)

*Wilcoxina verruculosa M. Zeng, Q. Zhao \& K.D. Hyde, Fungal Diversity 100: 163 (2020)

Wojnowiciella clematidis Phukhams. \& K.D. Hyde, Fungal Diversity 10.1007/s13225020-00448-4: 85 (2020)

Wrightoporia grandipora Decock \& Ryvarden, Syn. Fung. 42: 13 (2020)

*Xanthagaricus siamensis Yuan S. Liu \& S. Lumyong, Phytotaxa 437 (1): 17 (2020)

Xanthomendoza leoncita Bungartz \& Søchting, Plant and Fungal Systematics 65 (2): 569 (2020)

Xanthoria ibizaensis S.Y. Kondr. \& A.S. Kondr., Acta bot. hung. 62 (1-2): 96 (2020)

*Xenasmatella rhizomorpha C.L. Zhao, Phytotaxa 489 (2): 115 (2020)

*Xenasmatella tenuis C.L. Zhao, Phytotaxa 489 (2): 116 (2020)

*Xenasmatella xinpingensis C.L. Zhao, Phytotaxa 489 (2): 118 (2020)

Xenoacremonium brunneosporum Dayarathne, E.B.G. Jones \& K.D. Hyde, Mycosphere 11 (1): 113 (2020)

Xenoanthostomella chromolaenae Mapook \& K.D. Hyde, Fungal Diversity 100: 237 (2020)
Xenocylindrosporium margaritarum C.F.J. Spies, van Jaarsveld, Halleen \& L. Mostert, Persoonia 45: 210 (2020)

Xenodidymella camporesii D. Pem, Doilom \& K.D. Hyde, Fungal Diversity 100: 45 (2020) Xenodidymella clematidis Phukhams., Camporesi \& K.D. Hyde, Fungal Diversity 10.1007/s13225-020-00448-4: 30 (2020)

*Xenodidymella glycyrrhizicola L.W. Hou, L. Cai \& Crous, Stud. Mycol. 96: 388 (2020)

*Xenodidymella weymaniae Hern.-Restr., L.W. Hou, L. Cai \& Crous, MycoKeys 65: 89 (2020)

Xenomassariosphaeria clematidis Wanas., Phukhams., Camporesi \& K.D. Hyde, Fungal Diversity 10.1007/s13225-02000448-4: 67 (2020)

Xenomonodictys iranica Hern.-Restr., Karimi, Alizadeh \& T. Ghanbary, Persoonia 45: 401 (2020)

Xenonectriella nephromatis Pérez-Ort., Lichenologist 52 (2): 127 (2020)

Xenonectriella physciacearum F. Berger, E. Zimm. \& Brackel, Herzogia 33: 483 (2020)

Xenonectriella zimmermanni F. Berger \& Brackel, Herzogia 33: 486 (2020)

Xenoplectosphaerella clematidis Phukhams., Jayaward. \& K.D. Hyde, Fungal Diversity 10.1007/s13225-020-00448-4: 179 (2020)

Xenoroussoella triseptata Mapook \& K.D. Hyde, Fungal Diversity 101: 95 (2020)

Xerocomellus atropurpureus J.L. Frank, N. Siegel \& C.F. Schwarz, Fungal Systematics and Evolution 6: 276 (2020)

Xerocomellus diffractus N. Siegel, C.F. Schwarz \& J.L. Frank, Fungal Systematics and Evolution 6: 278 (2020)

Xerocomellus salicicola C.F. Schwarz, N. Siegel \& J.L. Frank, Fungal Systematics and Evolution 6: 283 (2020)

Xerophorus dominicanus Angelini, Vizzini \& Bizzi, Fungal Diversity 101: 243 (2020)

Xyladictyochaeta tristaniopsidis Crous, 
Persoonia 45: 289 (2020)

*Xylaria acericola H.X. Ma, Lar.N. Vasilyeva \& Yu Li, Phytotaxa 436 (1): 37 (2020)

*Xylaria ellisii J.B. Tanney, Seifert \& Y.M. Ju, Scientific Reports 10 (no. 4599): 9 (2020)

Xylaria fabacearum R.H. Perera, E.B.G. Jones \& K.D. Hyde, Mycosphere 11 (1): 2175 (2020)

Xylaria fabaceicola R.H. Perera, E.B.G. Jones \& K.D. Hyde, Mycosphere 11 (1): 2179 (2020)

Xylaria friabilis J. Fourn. \& Lechat, Ascomycete.org 12 (3): 110 (2020)

*Xylaria insolita Y.M. Ju, H.M. Hsieh \& J.C. Chou, Bot. Studies 61 (no. 11): 2 (2020)

Xylaria martinicensis J. Fourn. \& Lechat, Ascomycete.org 12 (3): 120 (2020)

Xylaria pallidocylindracea J. Fourn. \& Lechat, Ascomycete.org 12 (3): 137 (2020)

*Xylaria subescharoidea Y.M. Ju, H.M. Hsieh \& J.C. Chou, Bot. Studies 61 (no. 11): 4 (2020)

*Xylaria vinosa J. Fourn., Y.M. Ju \& Lechat, Ascomycete.org 12 (3): 156 (2020)

*Xylolentia reniformis C.G. Lin, K.D. Hyde \&
J.K. Liu, Fungal Diversity 10.1007/s13225020-00461-7: 93 (2020)

*Yamadamyces rosulatus Golubev \& Scorzetti ex Q.M. Wang, F.Y. Bai, M. Groenew. \& Boekhout, Stud. Mycol. 96: 138 (2020)

*Yamadamyces terricola Q.M. Wang, F.Y. Bai \& A.H. Li, Stud. Mycol. 96: 125 (2020)

Yamadazyma cocois Maksim., Glushakova, Thanh \& Kachalkin, Int. J. Syst. Evol. Microbiol. 10.1099/ijsem.0.004203: 5 (2020) Ypsilina buttingtonensis Crous, Wainhouse \& B. Douglas, Persoonia 44: 339 (2020)

Yunnanensis chromolaenae Mapook \& K.D. Hyde, Fungal Diversity 101: 84 (2020)

*Yurkovia longicylindrica Q.M. Wang, F.Y. Bai \& A.H. Li, Stud. Mycol. 96: 131 (2020)

Zanclospora bicolorata R.F. Castañeda, M. Villav. \& D. Sosa, Mycotaxon 135 (4): 896 (2020)

Zhuliangomyces pakistanicus Usman \& Khalid, Phytotaxa 443 (2): 202 (2020)

Zygotorulaspora cariocana J.D. Moreira, A.R.O. Santos, P.B. Morais, M.A. Lachance \& C.A. Rossa, Int. J. Syst. Evol. Microbiol. 70 (4): 2680 (2020)

\section{亚种 Subspecies}

Diatomophthora perforans subsp. destruens Buaya \& Thines, Mycol. Progr. 19 (5): 451 (2020)

Diatomophthora perforans subsp. norvegica Buaya \& Thines, Mycol. Progr. 19 (5): 451 (2020)

Diatomophthora
pleurosigmae
Progr. 19 (5): 451 (2020)

Melanconis marginalis subsp. europaea Jaklitsch \& Voglmayr, MycoKeys 63: 89 (2020)

Melanconis marginalis subsp. tirolensis Jaklitsch \& Voglmayr, MycoKeys 63: 99 (2020)

\section{变种 Varieties}

Cortinarius brunneocarpus var. microsporus

J. Khan, Pl. Syst. Evol. 306 (no. 76): 5 (2020)

Cortinarius natalis var. albocaeruleus E. Campo, Journal des JEC 22: 4 (2020)
Inocybe pararubens var. padjelantae E. Larss. \& Vauras, Karstenia 58 (1): 35 (2020)

Leptonia cyanea var. occidentalis Largent, Mycotaxon 135 (4): 794 (2020) 
Pertusaria microstoma var. deficiens A.W. Archer \& Elix, Australas. Lichenol. 86: 18 (2020)

Phaeoclavulina flaccida var. fraceolens Franchi \& M. Marchetti, Index Fungorum 467: 3 (2020)

Rhodocybe luteocinnamomea var. fulva T.J. Baroni \& Angelini, Mycol. Progr. 19 (10): 1093 (2020)

Trichophyton benhamiae var. luteum
Čmoková \& Hubka, Fungal Diversity 104: 356 (2020)

Tuber brumale var. gorii Della Maggiora, Micologia Toscana 2: 52 (2020)

Xylaria martinicensis var. martinicensis $\mathrm{J}$. Fourn. \& Lechat, Ascomycete.org 12 (3): 120 (2020)

Xylaria martinicensis var. microspora J. Fourn. \& Lechat, Ascomycete.org 12 (3): 123 (2020)

\section{变型 Forms}

Amanita albogrisescens f. subglobispora Hanss, Bull. Soc. mycol. Fr. 133: 107 (2020) Artomyces pyxidatus $\mathrm{f}$. luteovirescens Franchi \& M. Marchetti, Index Fungorum 467: 1 (2020)

Clavulina coralloides f. coriobrunnescens Franchi \& M. Marchetti, Index Fungorum 467: 1 (2020)

Clavulina coralloides f. griseorosata Franchi \& M. Marchetti, Index Fungorum 467: 1 (2020)

Ramaria luteoaurantiaca f. luteoaurantiaca Franchi \& M. Marchetti, Index Fungorum
433: 1 (2020)

Ramaria pallida f. lactea Franchi \& M. Marchetti, Index Fungorum 467: 6 (2020)

Ramaria schildii f. griseoavellanea E. Campo, Franchi \& M. Marchetti, Index Fungorum 467: 6 (2020)

Ramaria stricta f. sambucina Franchi \& M. Marchetti, Index Fungorum 467: 7 (2020)

Suillus americanus f. pseudosibiricus Klofac, Öst. Z. Pilzk. 28: 20 (2020)

Xerocomus ferrugineus f. aurantiiporus Klofac \& Krisai, Öst. Z. Pilzk. 28: 10 (2020)

\section{组合 Combinations}

*Acarospora bolleana (H. Magn.) K. Knudsen, J.N. Adams, Kocourk. \& Y. Wang, Bryologist 123 (1): 27 (2020)

*Acaulium peruvianum (Udagawa \& Furuya) Lei Su, Scientific Reports 10 (no. 10302): 4 (2020)

*Acaulium retardatum (Udagawa \& T. Muroi) Lei Su, Scientific Reports 10 (no. 10302): 6 (2020)

Acidotalaromyces lignorum (Stolk) Houbraken, Frisvad \& Samson, Stud. Mycol. 95: 86 (2020)

Acrocordia endobrya (Döbbeler \& Poelt) Lücking \& Aptroot, Fungal Diversity 10.1007/s13225-020-00462-6: 86 (2020)

Actinomortierella ambigua (B.S. Mehrotra) Vandepol \& Bonito, Fungal Diversity 10.1007/s13225-020-00455-5: 13 (2020)

Actinomortierella capitata (Marchal) Vandepol \& Bonito, Fungal Diversity 10.1007/s13225-020-00455-5: 13 (2020)

Actinomortierella wolfii (B.S. Mehrotra \& Baijal) Vandepol \& Bonito, Fungal Diversity 10.1007/s13225-020-00455-5: 13 (2020)

*Akanthomyces pissodis (Kope \& I. Leal) W.H. Chen, Y.F. Han \& Z.Q. Liang, Phytotaxa 459 (2): 120 (2020) 
Albocoprinus ealaensis (Beeli) Voto, Rivista Micologica Romana, Boll. AMER 110 (2): 90 (2020)

Allodiatrype thailandica (R.H. Perera, Jian K. Liu \& K.D. Hyde) Konta \& K.D. Hyde, Mycosphere 11 (1): 253 (2020)

Alloleptosphaeria iridicola (Crous \& Denman) Voglmayr, MycoKeys 66: 14 (2020)

Alyxoria xerica (Torrente \& Egea) Van Haluwyn \& Cl. Roux, Catalogue des lichens et champignons lichénicoles de France métropolitaine. 3e édition revue et augmentée 1: 1285 (2020)

Amanita albogrisescens f. cinereifolia (Contu) Hanss, Bull. Soc. mycol. Fr. 133: 107 (2020)

Amanita albogrisescens f. pseudolactea (Contu) Hanss, Bull. Soc. mycol. Fr. 133: 106 (2020)

\section{Amanita brunneofuliginea f.} ochraceomaculata (Neville, Poumarat \& Fraiture) P.-A. Moreau, Bull. Soc. mycol. Fr. 133: 119 (2020)

Amanita brunneofuliginea f. ochraceopallida (Contu) Hanss, Bull. Soc. mycol. Fr. 133: 119 (2020)

Amanita inzengae (Neville \& Poumarat) Di Rita, Atzeni, Tudino, Tatti \& P. Alvarado, Index Fungorum 455: 1 (2020)

Amanita lividopallescens f. malleata (Piane ex Bon) Hanss, Bull. Soc. mycol. Fr. 133: 125 (2020)

Amaurodermellus ovisporum (Gomes-Silva, Ryvarden \& Gibertoni) Costa-Rezende, Drechsler-Santos \& Góes-Neto, Mycol. Progr. 19 (8): 733 (2020)

Amphisphaeria fuckelii (G.H. Otth) Samarak., Maharachch. \& K.D. Hyde, Journal of Fungi 6 (3): 16 (2020)

Amphisphaeria qujingensis (Dissan., J.C. Kang \& K.D. Hyde) Samarak., Maharachch. \& K.D. Hyde, Journal of Fungi 6 (3): 19 (2020)

Amphisphaeria sambuci (Jaklitsch \&
Voglmayr) Samarak., Maharachch. \& K.D. Hyde, Journal of Fungi 6 (3): 19 (2020)

Amphisphaeria uniseptata (K.M. Tsui, K.D. Hyde \& Hodgkiss) Samarak., Maharachch. \& K.D. Hyde, Journal of Fungi 6 (3): 20 (2020)

*Anasporidesmiella angustobasilaris (Hol.Jech.) K. Zhang, R.F. Castañeda, Heredia \& Jian Ma, Mycotaxon 135 (4): 724 (2020)

Angiactis banksiae (Müll. Arg.) Kantvilas \& Stajsic, Muelleria 38: 72 (2020)

Angiopsora apoda (Har. \& Pat.) Aime \& McTaggart, Fungal Systematics and Evolution 7: 41 (2020)

Angiopsora chusqueae (Pardo-Card.) Aime \& McTaggart, Fungal Systematics and Evolution 7: 41 (2020)

Angiopsora paspalicola (Henn.) Aime \& McTaggart, Fungal Systematics and Evolution 7: 41 (2020)

Anthodidymella clematidis (Woudenb., Spiers \& Gruyter) Phukhams. \& K.D. Hyde, Fungal Diversity 10.1007/s13225-020-00448-4: 26 (2020)

Anthodidymella vitalbina (Petr.) Phukhams. \& K.D. Hyde, Fungal Diversity 10.1007/s13225-020-00448-4: 28 (2020)

Anthosulcatispora brunnea (Y. Chen) Phukhams. \& K.D. Hyde, Fungal Diversity 10.1007/s13225-020-00448-4: 119 (2020)

*Antrodiella descendens (Corner) C.L. Zhao \& Y.C. Dai, Fungal Diversity 10.1007/s13225-020-00461-7: 142 (2020)

Antrodiopsis monomitica (Yuan Y. Chen) Audet, Index Fungorum 452: 1 (2020)

Aphanodesmium gabretae (Koukol \& Koláŕová) Réblová \& Hern.-Restr., Stud. Mycol. 95: 442 (2020)

Appendiculina gregaria (W. Rossi) Haelew. \& Aime, Sydowia 72: 325 (2020)

Appendiculina scaptomyzae (Thaxt.) Haelew. \& Aime, Sydowia 72: 325 (2020)

*Aquihelicascus thalassioideus (K.D. Hyde \& 
Aptroot) W. Dong \& H. Zhang, Fungal Diversity $\quad 10.1007 / \mathrm{s} 13225-020-00463-5$ : 145 (2020)

*Aquimassariosphaeria typhicola (P. Karst.) W. Dong \& Doilom, Fungal Diversity 10.1007/s13225-020-00463-5: 108 (2020)

Arachnopeziza sphagniseda (Velen.) $\mathrm{T}$. Kosonen, Huhtinen \& K. Hansen, Persoonia 46: 43 (2020)

Araucariomyces balansae (Cornu) McTaggart, R.G. Shivas \& Aime, Fungal Systematics and Evolution 7: 34 (2020)

Araucariomyces fragiformis (Ces.) McTaggart, R.G. Shivas \& Aime, Fungal Systematics and Evolution 7: 34 (2020)

Areotheca ambigua (Sacc.) Y. Marín \& Stchigel, Microorganisms 8 (9, no. 1430): 14 (2020)

Areotheca areolata (N. Lundq.) Y. Marín, A.N. Mill. \& Stchigel, Microorganisms 8 (9, no. 1430): 13 (2020)

Arrhenia tillii (Krisai \& Noordel.) Krisai \& I. Saar, Sydowia 73: 156 (2020)

Arthonia rimularum (Wedd.) Monnat \& $\mathrm{Cl}$. Roux, Catalogue des lichens et champignons lichénicoles de France métropolitaine. 3e édition revue et augmentée 1: 1285 (2020)

Arthroderma magnisporum (Stchigel, Cano, Mac Cormack \& Guarro) Rahul Sharma \& Hubka, Medical Mycol. myaa057: 7 (2020)

Arthroderma oceanitis (Stchigel, Cano, Archuby \& Guarro) Rahul Sharma \& Hubka, Medical Mycol. myaa057: 9 (2020)

Arthroderma terrestre (Durie \& D. Frey) Kučerová \& Hubka, Medical Mycol. myaa057: 9 (2020)

*Ascagilis guttulaspora (Qing Tian, Y.Z. Lu \& K.D. Hyde) W. Dong, Doilom \& K.D. Hyde, Fungal Diversity 10.1007/s13225-02000463-5: 29 (2020)

*Ascagilis queenslandica (Dayarathne, Fryar \& K.D. Hyde) W. Dong, Doilom \& K.D. Hyde, Fungal Diversity 10.1007/s13225-
020-00463-5: 29 (2020)

*Ascagilis seychellensis (K.D. Hyde \& S.W. Wong) W. Dong, Doilom \& K.D. Hyde, Fungal Diversity 10.1007/s13225-02000463-5: 29 (2020)

*Ascagilis sunyatsenii (Inderb.) W. Dong, Doilom \& K.D. Hyde, Fungal Diversity 10.1007/s13225-020-00463-5: 31 (2020)

*Ascochyta astragalina (Rehm) L.W. Hou, L. Cai \& Crous, Stud. Mycol. 96: 379 (2020)

*Ascochyta koolunga (Davidson, Hartley, Priest, Krysinska-Kaczmarek, Herdina, McKay \& Scott) L.W. Hou, L. Cai \& Crous, Stud. Mycol. 96: 381 (2020)

Ascocoryne lilacina (Fr.) Baral, Helleman, Matočec, I. Kušan, Polhorský \& E. Weber, Index Fungorum 428: 1 (2020)

Ascospirella lutea (Zukal) Houbraken, Frisvad \& Samson, Stud. Mycol. 95: 86 (2020)

Aspergillus ser. Inflati (Stolk \& Samson) Houbraken \& Frisvad, Stud. Mycol. 95: 41 (2020)

Aspicilia flavoreagens (Asta \& Cl. Roux) $\mathrm{Cl}$. Roux, Catalogue des lichens et champignons lichénicoles de France métropolitaine. 3e édition revue et augmentée 1: 1285 (2020)

Asproinocybe fucata (K.B. Vrinda \& C.K. Pradeep) Ralaiv., Niskanen \& Liimat., Mycol. Progr. 19: 1298 (2020)

Aureoboletus betula (Schwein.) M. Kuo \& B. Ortiz, Mycologia 112 (1): 205 (2020)

Backusella dispersa (Hagem) Urquhart \& Douch, Persoonia 46: 16 (2020)

*Balsamia guozigouensis (L. Fan \& Y.Y. Xu) L. Fan \& Y.Y. Xu, MycoKeys 67: 90 (2020) *Balsamia luyashanensis (L. Fan \& Y.Y. Xu) L. Fan \& Y.Y. Xu, MycoKeys 67: 90 (2020) Bipolaris gigantea (Heald \& F.A. Wolf) B. Lane, Stricker, M.E. Sm., S.L. Flory \& Harmon, Mycologia 112 (5): 926 (2020)

Blastenia festivella (Nyl.) Vondrák, Journal of Systematics and Evolution 58 (3): 317 (2020) Blastenia subathallina (H. Magn.) Arup \& 
Vondrák, Journal of Systematics and Evolution 58 (3): 322 (2020)

Bogoriella chiquitana (Flakus, Kukwa \& Aptroot) Lücking, R. Miranda \& Aptroot, Fungal Diversity 10.1007/s13225-02000462-6: 165 (2020)

Bogoriella isthmospora (Aptroot) Lücking, $\mathrm{R}$. Miranda \& Aptroot, Fungal Diversity 10.1007/s13225-020-00462-6: 166 (2020)

*Bogoriella pandanicola (S.N. Zhang \& K.D. Hyde) S.N. Zhang, Lücking \& Aptroot, Fungal Diversity 10.1007/s13225-02000462-6: 166 (2020)

Bogoriella rubrostoma (Aptroot) Lücking, R. Miranda \& Aptroot, Fungal Diversity 10.1007/s13225-020-00462-6: 166 (2020)

Boletinus ampliporus (Peck) Klofac, Öst. Z. Pilzk. 28: 19 (2020)

Botrytis cirsii-spinosissimi (Senn-Irlet) Baral, Index Fungorum 454: 2 (2020)

Bourdotiella crustula (L.W. Mill.) K.H. Larss. \& Ryvarden, Syn. Fung. 40: 113 (2020)

Britzelmayria multipedata (Peck) D. Wächt. \& A. Melzer, Mycol. Progr. 19 (11): 1213 (2020)

Britzelmayria supernula (Britzelm.) D. Wächt. \& A. Melzer, Mycol. Progr. 19 (11): 1213 (2020)

Brocchiosphaera brocchiata (Tubaki) K. Yamag., Chuaseehar. \& Nakagiri, Mycoscience 61 (6): 279 (2020)

Brocchiosphaera microspora (R.F. Castañeda \& W.B. Kendr.) K. Yamag., Chuaseehar. \& Nakagiri, Mycoscience 61 (6): 279 (2020)

Bryopistillaria sagittiformis (Pat.) Olariaga, Huhtinen, Læssøe, J.H. Petersen \& K. Hansen, Stud. Mycol. 96: 177 (2020)

Bryoscyphus phascoides (Fr.) Baral, Index Fungorum 428: 1 (2020)

Bryoscyphus rhytidiadelphi (Svrček) Baral, Index Fungorum 428: 1 (2020)

Bryostigma apotheciorum (A. Massal.) S.Y. Kondr. \& Hur, Acta bot. hung. 62 (1-2): 99
(2020)

Bryostigma biatoricola (Ihlen \& Owe-Larss.) S.Y. Kondr. \& Hur, Acta bot. hung. 62 (1-2): 99 (2020)

Bryostigma dokdoense (S.Y. Kondr., Lőkös, B.G. Lee, J.J. Woo \& Hur) S.Y. Kondr. \& Hur, Acta bot. hung. 62 (1-2): 99 (2020)

Bryostigma epiphyscium (Nyl.) S.Y. Kondr. \& Hur, Acta bot. hung. 62 (1-2): 99 (2020)

Bryostigma lapidicola (Taylor) S.Y. Kondr. \& Hur, Acta bot. hung. 62 (1-2): 100 (2020)

Bryostigma lobariellae (Etayo) S.Y. Kondr. \& Hur, Acta bot. hung. 62 (1-2): 100 (2020)

Bryostigma molendoi (Heufl. ex Frauenf) S.Y. Kondr. \& Hur, Acta bot. hung. 62 (1-2): 100 (2020)

Bryostigma neglectulum (Nyl.) S.Y. Kondr. \& Hur, Acta bot. hung. 62 (1-2): 100 (2020)

Bryostigma parietinarium (Hafellner \& A. Fleischhacker) S.Y. Kondr. \& Hur, Acta bot. hung. 62 (1-2): 100 (2020)

Bryostigma peltigerinum (Almq.) S.Y. Kondr. \& Hur, Acta bot. hung. 62 (1-2): 100 (2020)

Bryostigma phaeophysciae (Grube \& Matzer) S.Y. Kondr. \& Hur, Acta bot. hung. 62 (1-2): 100 (2020)

Bryostigma stereocaulinum (Ohlert) S.Y. Kondr. \& Hur, Acta bot. hung. 62 (1-2): 100 (2020)

Buellia bispora (Sheard) Brodo \& Sheard, Opuscula Philolichenum 19: 167 (2020)

*Butyriboletus rubrus (M. Zang) Kui Wu, Gang Wu \& Zhu L. Yang, Acta Edulis Fungi 72 (2): 96 (2020)

Cadophora bubakii (Laxa) Damm \& S. Bien, MycoKeys 63: 134 (2020)

Cadophora bubakii (Laxa) Damm \& S. Bie, MycoKeys 69: 112 (2020)

Cadophora dextrinospora (Korf) Koukol \& Maciá-Vicente, IMA Fungus 11 (no. 16): 9 (2020)

Calicium brachysporum (Nádv.) K. Knudsen, Kocourk. \& Lendemer, Bull. Calif. Lichen 
Soc. 26 (2): 52 (2020)

Calliderma callidermum (Romagn.) Largent, Mycotaxon 135 (4): 793 (2020)

Callistosporium brunescens (Earle) Vizzini, Consiglio \& M. Marchetti, Fungal Diversity 101: 235 (2020)

Callistosporium hesleri (H.E. Bigelow) Vizzini, Matheny, Consiglio \& M. Marchetti, Fungal Diversity 101: 235 (2020)

Callistosporium imbricatum (T.J. Baroni, Lodge \& D.L. Lindner) Vizzini, Consiglio \& M. Marchetti, Fungal Diversity 101: 235 (2020)

Callistosporium praemultifolium (Murrill) Vizzini, Consiglio \& M. Marchetti, Fungal Diversity 101: 234 (2020)

Caloboletus polygonius var. ereticulatus (Estadès \& Lannoy) Chapon \& P. Roux, Bull. Mycol. Bot. Dauphiné-Savoie 236: 43 (2020)

*Calophoma vincetoxici (De Not.) L.W. Hou, L. Cai \& Crous, Stud. Mycol. 96: 377 (2020) Caloplaca lecideina (Müll. Arg.) Cl. Roux, Catalogue des lichens et champignons lichénicoles de France métropolitaine. 3e édition revue et augmentée 1: 1286 (2020)

Calycellina separabilis (P. Karst.) Baral, Index Fungorum 428: 1 (2020)

Calycina carneorosea (Cooke \& Harkn.) Baral, Monogr. Orbiliomycetes: 1664 (2020)

*Camporesiomyces patagonicus (R.M. Sánchez, A.N. Mill. \& Bianchin) D.P. Wei, Wanas. \& K.D. Hyde, Fungal Diversity 10.1007/s13225-020-00439-5: 138 (2020)

*Camporesiomyces vaccinii (Carris) D.P. Wei \& K.D. Hyde, Fungal Diversity 100: 142 (2020)

Camposporium lycopodiellae (Crous \& R.K. Schumach.) Tibpromma \& K.D. Hyde, Fungal Diversity 100: 81 (2020)

Candelinella deppeanae (M. Westberg) S.Y. Kondr., Acta bot. hung. 62 (3-4): 304 (2020)

Candelinella makarevichiae (S.Y. Kondr., Lökös \& Hur) S.Y. Kondr., Acta bot. hung.
62 (3-4): 304 (2020)

Candolleomyces aberdarensis (A. Melzer, Kimani \& R. Ullrich) D. Wächt. \& A. Melzer, Mycol. Progr. 19 (11): 1230 (2020) Candolleomyces albipes (Murrill) D. Wächt. \& A. Melzer, Mycol. Progr. 19 (11): 1230 (2020)

Candolleomyces badhyzensis (Kalamees) D. Wächt. \& A. Melzer, Mycol. Progr. 19 (11): 1231 (2020)

Candolleomyces badiophyllus (Romagn.) D. Wächt. \& A. Melzer, Mycol. Progr. 19 (11): 1231 (2020)

Candolleomyces bivelatus (Contu) D. Wächt. \& A. Melzer, Mycol. Progr. 19 (11): 1232 (2020)

Candolleomyces cacao (Desjardin \& B.A. Perry) D. Wächt. \& A. Melzer, Mycol. Progr. 19 (11): 1233 (2020)

Candolleomyces caespitosus (Murrill) D. Wächt. \& A. Melzer, Mycol. Progr. 19 (11): 1234 (2020)

Candolleomyces candolleanus (Fr.) D. Wächt. \& A. Melzer, Mycol. Progr. 19 (11): 1233 (2020)

Candolleomyces efflorescens (Sacc.) D. Wächt. \& A. Melzer, Mycol. Progr. 19 (11): 1233 (2020)

Candolleomyces fimicola (Atri, M. Kaur \& Amand. Kaur) D. Wächt. \& A. Melzer, Mycol. Progr. 19 (11): 1233 (2020)

Candolleomyces floccosus (Earle) D. Wächt. \& A. Melzer, Mycol. Progr. 19 (11): 1233 (2020)

Candolleomyces graminus (Kalamees) D. Wächt. \& A. Melzer, Mycol. Progr. 19 (11): 1233 (2020)

Candolleomyces halophilus (Esteve-Rav. \& Enderle) D. Wächt. \& A. Melzer, Mycol. Progr. 19 (11): 1233 (2020)

Candolleomyces leucotephrus (Berk. \& Broome) D. Wächt. \& A. Melzer, Mycol. Progr. 19 (11): 1233 (2020) 
Candolleomyces luteopallidus (A.H. Sm.) D. Wächt. \& A. Melzer, Mycol. Progr. 19 (11): 1234 (2020)

Candolleomyces paecilospermus (Pacioni) D. Wächt. \& A. Melzer, Mycol. Progr. 19 (11): 1234 (2020)

Candolleomyces pseudocandolleanus (A.H. Sm.) D. Wächt. \& A. Melzer, Mycol. Progr. 19 (11): 1234 (2020)

Candolleomyces rupchandii (A.H. Sm.) D. Wächt. \& A. Melzer, Mycol. Progr. 19 (11): 1234 (2020)

Candolleomyces secotioides (G. Moreno, Heykoop, Esqueda \& Olariaga) D. Wächt. \& A. Melzer, Mycol. Progr. 19 (11): 1234 (2020)

Candolleomyces singeri (A.H. Sm.) D. Wächt. \& A. Melzer, Mycol. Progr. 19 (11): 1234 (2020)

Candolleomyces subsingeri (T. Bau \& J.Q. Yan) D. Wächt. \& A. Melzer, Mycol. Progr. 19 (11): 1234 (2020)

Candolleomyces sulcatotuberculosus (J. Favre) D. Wächt. \& A. Melzer, Mycol. Progr. 19 (11): 1234 (2020)

Candolleomyces trinitatensis (R.E.D. Baker \& W.T. Dale) D. Wächt. \& A. Melzer, Mycol. Progr. 19 (11): 1234 (2020)

Candolleomyces tuberculatus (Pat.) D. Wächt. \& A. Melzer, Mycol. Progr. 19 (11): 1234 (2020)

Candolleomyces typhae (Kalchbr.) D. Wächt. \& A. Melzer, Mycol. Progr. 19 (11): 1234 (2020)

Cantharellus isaloensis (Buyck \& Eyssart.) Buyck, Cryptog. Mycol. 41 (10): 169 (2020)

Cantharellus littoralis (Buyck \& Randrianj.) Buyck, Cryptog. Mycol. 41 (10): 169 (2020) *Capillidium adiaeretum (Drechsler) B. Huang \& Y. Nie, MycoKeys 66: 63 (2020)

*Capillidium bangalorense (Sriniv. \& Thirum.) B. Huang \& Y. Nie, MycoKeys 66: 71 (2020) *Capillidium denaeosporum (Drechsler) B.
Huang \& Y. Nie, MycoKeys 66: 71 (2020)

*Capillidium heterosporum (Drechsler) B. Huang \& Y. Nie, MycoKeys 66: 62 (2020)

*Capillidium lobatum (Sriniv. \& Thirum.) B. Huang \& Y. Nie, MycoKeys 66: 71 (2020)

*Capillidium pumilum (Drechsler) B. Huang \& Y. Nie, MycoKeys 66: 71 (2020)

*Capillidium rhysosporum (Drechsler) B. Huang \& Y. Nie, MycoKeys 66: 72 (2020)

Capitotricha attenuans (Nyl.) E. Rubio, J. Linde \& Baral, Ascomycete.org 12 (5): 207 (2020)

Capitotricha scabrovillosa (W. Phillips) Baral \& Järv, Index Fungorum 428: 1 (2020)

*Capnodium aciculiforme (Cif., Bat. \& Nascim.) W.J. Li \& K.D. Hyde, Fungal Diversity 100: 372 (2020)

*Capnodium gardeniarum (Bat. \& Cif.) W.J. Li \& K.D. Hyde, Fungal Diversity 100: 372 (2020)

Carbacanthographis garoana (Nagarkar \& Patw.) Pushpi Singh \& Kr. P. Singh, Lichenologist 52 (3): 251 (2020)

Carestiella schizoxyloides (Ellis \& Everh.) Baral, Index Fungorum 428: 1 (2020)

Catillaria laevis (M. Brand \& van den Boom) Cl. Roux, Catalogue des lichens et champignons lichénicoles de France métropolitaine. 3e édition revue et augmentée 1: 1286 (2020)

Cephalotelium evansii (Syd. \& P. Syd.) Aime \& McTaggart, Fungal Systematics and Evolution 7: 39 (2020)

Cephalotelium neocaledoniense (B. Huguenin) Aime \& McTaggart, Fungal Systematics and Evolution 7: 39 (2020)

Cephalotelium xanthophloeae (M. Ebinghaus, W. Maier \& Begerow) Aime \& McTaggart, Fungal Systematics and Evolution 7: 39 (2020)

Cercosporella bundelkhandae (S. Shrivast., N. Verma \& A.N. Rai) Raghv. Singh, S.K. Verma, S. Yadav \& Sh. Kumar, Mycotaxon 
135 (2): 317 (2020)

Ceropsora weirii (H.S. Jacks.) Aime \& McTaggart, Fungal Systematics and Evolution 7: 36 (2020)

*Chaetasbolisia eupatorii (Died.) L.W. Hou, L. Cai \& Crous, Stud. Mycol. 96: 333 (2020)

Chaetocapnodium microglobulosum (Bat. \& Cif.) S.A. Khodaparast, Mycol. Progr. 19 (2): 163 (2020)

Chaetocapnodium philippinense (Hongsanan \& K.D. Hyde) Abdollahz. \& Crous, Stud. Mycol. 95: 399 (2020)

Chaetocapnodium placitae (Cheew. \& Crous) Abdollahz. \& Crous, Stud. Mycol. 95: 400 (2020)

Chaetodermella incrassata (Malençon) K.H. Larss. \& Ryvarden, Syn. Fung. 40: 114 (2020)

Chaetoscypha horoeka (P.R. Johnst.) Baral \& P.R. Johnst., Index Fungorum 428: 1 (2020)

Chaetoscypha mahinapua (P.R. Johnst.) Baral \& P.R. Johnst., Index Fungorum 428: 1 (2020)

Chaetoscypha palmicola (P.R. Johnst.) Baral \& P.R. Johnst., Index Fungorum 428: 1 (2020)

Chloridium macrocladum (Sacc.) Karun., Maharachch., C.H.Kuo \& K.D.Hyde, Fungal Diversity 10.1007/s13225-020-00461-7: 74 (2020)

Chromelosporiopsis carnea (Schumach.) Hennebert, Mycotaxon 135 (3): 689 (2020)

Chromelosporiopsis coerulescens (Bonord.) Hennebert, Mycotaxon 135 (3): 695 (2020)

Chromelosporium herbicola (Ellis \& Dearn.) Hennebert, Mycotaxon 135 (3): 673 (2020)

Chromolaenicola siamensis (Jayasiri, E.B.G. Jones \& K.D. Hyde) Mapook \& K.D. Hyde, Fungal Diversity 101: 26 (2020)

Cistella improvisa (P. Karst.) Baral, Index Fungorum 428: 1 (2020)

Cladorrhinum coprophilum (Fr.) Y. Marin, A.N. Mill. \& Stchigel, Microorganisms 8 (9, no. 1430): 19 (2020)

Cladorrhinum tomentosum (Speg.) Y. Marin $\&$ Stchigel, Microorganisms 8 (9, no. 1430): 20 (2020)

Clavulina coralloides f. lutea (Bull.) Franchi \& M. Marchetti, Index Fungorum 437: 1 (2020)

Clavulinopsis corniculata f. brunneipes (Schild) Franchi \& M. Marchetti, Index Fungorum 437: 1 (2020)

Clinoconidium inouyei (Henn. \& Shirai) Kakish., S. Shibata \& Hirooka, Phytotaxa 450 (2): 206 (2020)

Clitocella ammophila (Malençon) Consiglio, Index Fungorum 430: 1 (2020)

Clitocella nigrescens (Maire) Consiglio, Index Fungorum 430: 1 (2020)

Clitopiloides costata (Fr.) Largent, Mycotaxon 135 (4): 793 (2020)

Clitopiloides cyathus (Romagn. \& Gilles) Largent, Mycotaxon 135 (4): 793 (2020)

Clonostachys ambigua (Penz. \& Sacc.) Forin \& Vizzini, Persoonia 45: 234 (2020)

Clonostachys granuligera (Starbäck) Forin \& Vizzini, Persoonia 45: 240 (2020)

Clonostachys pallens (Penz. \& Sacc.) Forin \& Vizzini, Persoonia 45: 234 (2020)

Clonostachys squamuligera (Sacc.) Forin \& Vizzini, Persoonia 45: 245 (2020)

*Colacogloea subericola (Belloch, Villa-Carv., Álv.-Rodríg. \& Coque) Q.M. Wang \& F.Y. Bai, Stud. Mycol. 96: 132 (2020)

Colletotrichum cigarro (B.S. Weir \& P.R. Johnst.) A. Cabral \& P. Talhinhas, Plants 9 (4, no. 502): 12 (2020)

Commelinaceomyces aneilematis (S. Ito) E. Tanaka, Mycologia 112 (3): 655 (2020)

Commelinaceomyces burkillii (Syd., P. Syd. \& E.J. Butler) E. Tanaka, Mycologia 112 (3): 657 (2020)

Commelinaceomyces nawaschinii (Racib.) E. Tanaka, Mycologia 112 (3): 658 (2020)

Commelinaceomyces rwandensis ( $\mathrm{T}$. 
Majewski \& K.A. Nowak) E. Tanaka, Mycologia 112 (3): 658 (2020)

Coniocarpon cuspidans (Nyl.) Moen, Frisch \& Grube, MycoKeys 62: 41 (2020)

Coprinellus carbonicola (Singer) Voto, Rivista Micologica Romana, Boll. AMER 110 (2): 89 (2020)

Coprinellus castaneus (Berk. \& Broome) Voto, Rivista Micologica Romana, Boll. AMER 110 (2): 89 (2020)

Coprinellus plicatiloides (Buller) Voto, Boll. Assoc. Micol. Ecol. Romana 109 (1): 11 (2020)

Coprinellus sclerobasidium (Singer) Voto, Rivista Micologica Romana, Boll. AMER 110 (2): 89 (2020)

Coprinellus sect. Flocculosi (Citérin) D. Wächt. \& A. Melzer, Mycol. Progr. 19 (11): 1197 (2020)

Coprinellus subangularis (Thiers) Voto, Rivista Micologica Romana, Boll. AMER 110 (2): 89 (2020)

Coprinellus subrenispermus (Singer) Voto, Rivista Micologica Romana, Boll. AMER 110 (2): 89 (2020)

Coprinellus venustus (McKnight \& P. Allison) Voto, Rivista Micologica Romana, Boll. AMER 110 (2): 89 (2020)

Coprinopsis caracasensis (Dennis) Voto, Boll. Assoc. Micol. Ecol. Romana 109 (1): 11 (2020)

Coprinopsis discipes (Pat.) Voto, Boll. Assoc. Micol. Ecol. Romana 109 (1): 11 (2020)

Coprinopsis fagnani (Raithelh.) Voto, Boll. Assoc. Micol. Ecol. Romana 109 (1): 11 (2020)

Coprinopsis gelatinosa (D.A. Reid \& Eicker) Voto, Boll. Assoc. Micol. Ecol. Romana 109 (1): 11 (2020)

Coprinopsis hypsizyga (Singer) Voto, Rivista Micologica Romana, Boll. AMER 110 (2): 89 (2020)

Coprinopsis murina (Kalchbr.) Voto, Rivista
Micologica Romana, Boll. AMER 110 (2): 89 (2020)

Coprinopsis rhizophora (Kawam. ex Hongo \& K. Yokoy.) D.J. Schaf. \& B. Douglas, Field Mycology 21 (1): 6 (2020)

Coprinopsis saccospora (Singer) Voto, Rivista Micologica Romana, Boll. AMER 110 (2): 89 (2020)

Coprinopsis sect. Picaceae (Penn.) D. Wächt. \& A. Melzer, Mycol. Progr. 19 (11): 1254 (2020)

Coprinopsis subcurta (Thiers) Voto, Rivista Micologica Romana, Boll. AMER 110 (2): 89 (2020)

Coprinopsis subdomestica (Murill) D. Wächt. \& A. Melzer, Mycol. Progr. 19 (11): 1237 (2020)

Coprinus steppicola (Kalamees) Voto, Boll. Assoc. Micol. Ecol. Romana 109 (1): 11 (2020)

Cortinarius colorius (Bidaud) Niskanen, Dima \& Liimat., Fungal Diversity 10.1007/s13225-020-00459-1: 34 (2020)

Cortinarius iners (Bidaud) Liimat., Dima \& Niskanen, Fungal Diversity 10.1007/s13225-020-00459-1: 34 (2020)

Cortinarius lilaceus (Schwalb) Para, Micol. Veg. Medit. 34: 134 (2020)

Cortinarius subsect. Colymbadini (Melot) Niskanen, Kytöv. \& Liimat., Index Fungorum 439: 8 (2020)

Cortinarius violaceovolvatus var. viola (Soop) A.R. Nilsen \& Orlovich, Mycologia 112 (3): 598 (2020)

Cresponea subpremnea (Kantvilas \& Vězda) Kantvilas, Lichenologist 52 (4): 282 (2020)

Cristataspora coffeata (Berk.) Robledo, CostaRezende \& de Madrignac Bonzi, Mycol. Progr. 19 (8): 734 (2020)

Cristataspora flavipora (Murrill) Robledo, Costa-Rezende \& de Madrignac Bonzi, Mycol. Progr. 19 (8): 735 (2020)

Crustoderma cryptocallimon (B. de Vries) 
K.H. Larss. \& Ryvarden, Syn. Fung. 40: 114 (2020)

*Cryphonectria citrina (Lar.N. Vassiljeva) C.M. Tian, N. Jiang \& Crous, Mycologia 112 (2): 280 (2020)

Cryptosphaeria bathurstensis (K.D. Hyde \& Rappaz) Dayar. \& K.D. Hyde, Cryptog. Mycol. 41 (3): 38 (2020)

Cubamyces flavidus (Lév.) Lücking, Willdenowia 50 (3): 396 (2020)

Cubamyces lactineus (Berk.) Lücking, Willdenowia 50 (3): 396 (2020)

Cubamyces menziesii (Berk.) Lücking, Willdenowia 50 (3): 396 (2020)

Cuphophyllus comosus (Bas \& Arnolds) Lodge, Boertm. \& E. Larss., Mycologia 112 (2): 445 (2020)

Cuphophyllus pseudopallidus (Hesler \& A.H. Sm.) Lodge, Boertm. \& E. Larss., Mycologia 112 (2): 449 (2020)

Curvularia cactivora (Petr.) Y. Marín \& Crous, Mycol. Progr. 19 (6): 568 (2020)

Curvularia patereae (M.R. Carranza) Y. Marín, Hern.-Restr. \& Crous, Mycol. Progr. 19 (6): 575 (2020)

Curvularia yamadana (Matsuura) B.W. Ferreira \& R.W. Barreto, Mycol. Progr. 19 (7): 717 (2020)

Cyathicula calathicola (Rehm) Baral, Index Fungorum 428: 1 (2020)

Cyathicula paludosa (Velen.) Baral, Index Fungorum 428: 1 (2020)

Cyathicula starbaeckii (Rehm) Hengstm., Mycol. Progr. 19 (6): 544 (2020)

Cyberloma acerinae (Jírovec) Minter, IMI Descr. Fungi Bact. 225 (no. 2241): 3 (2020)

Cyberloma psittaci (G. Casal, E. Matos, M.L. Teles-Grilo \& C. Azevedo) Minter, IMI Descr. Fungi Bact. 225 (no. 2241): 3 (2020) Cyphellophora aestiva (Nirenberg) Crous, Fungal Systematics and Evolution 7: 83 (2020)

Cyphellophora attinorum (Attili-Angelis,
Duarte, Stielow \& de Hoog) IturrietaGonzález, Gené \& Dania García, Persoonia 45: 345 (2020)

Cyphellophora capiguarae (Attili-Angelis, Duarte, Pagnocca \& M. de Vries) IturrietaGonzález, Gené \& Dania García, Persoonia 45: 345 (2020)

*Cystobasidium portillonense (Laich, Vaca \& Chávez) Q.M. Wang, F.Y. Bai, M. Groenew. \& Boekhout, Stud. Mycol. 96: 133 (2020)

*Cytospora viridistroma (Wehm.) C.M. Tian, N. Jiang \& Crous, Mycologia 112 (2): 281 (2020)

Dacryonaema macnabbii (D.A. Reid) J.C. Zamora \& S. Ekman, Persoonia 44: 192 (2020)

Daleomyces badioides (Donadini) Van Vooren, Ascomycete.org 12 (4): 185 (2020)

Daleomyces exogelatinosus (K. Hansen \& Sandal) Van Vooren, Ascomycete.org 12 (4): 185 (2020)

Daleomyces halophilus (Loizides, Agnello \& P. Alvarado) Van Vooren, Ascomycete.org 12 (4): 185 (2020)

Daleomyces petersii (Berk.) Van Vooren, Ascomycete.org 12 (4): 185 (2020)

Dasyscyphella chrysotexta (I. Kušan, Matočec \& Jadan) Baral, Index Fungorum 454: 2 (2020)

Dematioscypha castaneae (J.G. Han, Hosoya $\&$ H.D. Shin) Baral, Index Fungorum 428: 1 (2020)

Dematophora acutispora (Theiss.) C. Lamb., Wittstein \& M. Stadler, Stud. Mycol. 96: 11 (2020)

Dematophora arcuata (Petch) C. Lamb., Wittstein \& M. Stadler, Stud. Mycol. 96: 11 (2020)

Dematophora asperata (Massee ex Wakef.) C. Lamb., Wittstein \& M. Stadler, Stud. Mycol. 96: 11 (2020)

Dematophora beccariana (Ces.) C. Lamb., Wittstein \& M. Stadler, Stud. Mycol. 96: 11 
(2020)

Dematophora boedijnii (L.E. Petrini) C. Lamb., Wittstein \& M. Stadler, Stud. Mycol. 96: 11 (2020)

Dematophora bothrina (Berk. \& Broome) C. Lamb., Wittstein \& M. Stadler, Stud. Mycol. 96: 11 (2020)

Dematophora bunodes (Berk. \& Broome) C. Lamb., Wittstein \& M. Stadler, Stud. Mycol. 96: 14 (2020)

Dematophora buxi (Fabre) C. Lamb., Wittstein \& M. Stadler, Stud. Mycol. 96: 14 (2020)

Dematophora compacta (Takemoto) C. Lamb., Wittstein \& M. Stadler, Stud. Mycol. 96: 14 (2020)

Dematophora francisiae (L.E. Petrini) C. Lamb., Wittstein \& M. Stadler, Stud. Mycol. 96: 14 (2020)

Dematophora freycinetiae (L.E. Petrini) C. Lamb., Wittstein \& M. Stadler, Stud. Mycol. 96: 14 (2020)

Dematophora gigantea (Ellis \& Everh.) C. Lamb., Wittstein \& M. Stadler, Stud. Mycol. 96: 14 (2020)

Dematophora grantii (L.E. Petrini) C. Lamb., Wittstein \& M. Stadler, Stud. Mycol. 96: 14 (2020)

Dematophora hsiehae (L.E. Petrini) C. Lamb., Wittstein \& M. Stadler, Stud. Mycol. 96: 14 (2020)

Dematophora hughesii (L.E. Petrini) C. Lamb., Wittstein \& M. Stadler, Stud. Mycol. 96: 14 (2020)

Dematophora javaensis (L.E. Petrini) C. Lamb., Wittstein \& M. Stadler, Stud. Mycol. 96: 14 (2020)

Dematophora macdonaldii (Bres.) C. Lamb., Wittstein \& M. Stadler, Stud. Mycol. 96: 14 (2020)

Dematophora obregonii (L.E. Petrini) C. Lamb., Wittstein \& M. Stadler, Stud. Mycol. 96: 14 (2020)
Dematophora obtusiostiolata (L.E. Petrini) C. Lamb., Wittstein \& M. Stadler, Stud. Mycol. 96: 14 (2020)

Dematophora paraguayensis (Starbäck) C. Lamb., Wittstein \& M. Stadler, Stud. Mycol. 96: 14 (2020)

Dematophora pepo (Pat.) C. Lamb., Wittstein \& M. Stadler, Stud. Mycol. 96: 14 (2020)

Dematophora puiggariii (Pat.) C. Lamb., Wittstein \& M. Stadler, Stud. Mycol. 96: 14 (2020)

Dematophora pyramidalis (Lar.N. Vassiljeva) C. Lamb., Wittstein \& M. Stadler, Stud. Mycol. 96: 14 (2020)

Dematophora samuelsii (L.E. Petrini) C. Lamb., Wittstein \& M. Stadler, Stud. Mycol. 96: 14 (2020)

Dematophora siggersii (L.E. Petrini) C. Lamb., Wittstein \& M. Stadler, Stud. Mycol. 96: 14 (2020)

Dentipellis lindtneri (Pilát) K.H. Larss. \& Ryvarden, Syn. Fung. 40: 115 (2020)

Diaporthe destruens (Harter) Hirooka, Minosh. \& Rossman, Index Fungorum 429: 1 (2020)

Diarthonis spadicea (Leight.) Frisch, Ertz, Coppins \& P.F. Cannon, Revisions of British and Irish Lichens 1: 44 (2020)

Diatomophthora perforans (H.E. Petersen) Buaya \& Thines, Mycol. Progr. 19 (5): 449 (2020)

Diatomophthora perforans subsp. perforans (H.E. Petersen) Buaya \& Thines, Mycol. Progr. 19 (5): 449 (2020)

*Dichoporis bermudana (Tuck.) S.H. Jiang, Lücking \& Sérus., Fungal Diversity 10.1007/s13225-020-00462-6: 128 (2020)

*Dichoporis brevis (Bricaud \& Cl. Roux) S.H. Jiang, Lücking \& Sérus., Fungal Diversity 10.1007/s13225-020-00462-6: 128 (2020)

*Dichoporis connivens (R.C. Harris) S.H. Jiang, Lücking \& Sérus., Fungal Diversity 10.1007/s13225-020-00462-6: 128 (2020)

*Dichoporis dichosporidii (Etayo) S.H. Jiang, 
Lücking \& Sérus., Fungal Diversity 10.1007/s13225-020-00462-6: 128 (2020)

*Dichoporis elixii (P.M. McCarthy) S.H. Jiang, Lücking \& Sérus., Fungal Diversity 10.1007/s13225-020-00462-6: 128 (2020)

*Dichoporis fractans (P.M. McCarthy) S.H. Jiang, Lücking \& Sérus., Fungal Diversity 10.1007/s13225-020-00462-6: 128 (2020)

*Dichoporis maritima (H. Harada) S.H. Jiang, Lücking \& Sérus., Fungal Diversity 10.1007/s13225-020-00462-6: 128 (2020)

*Dichoporis minutula (P.M. McCarthy) S.H. Jiang, Lücking \& Sérus., Fungal Diversity 10.1007/s13225-020-00462-6: 128 (2020)

*Dichoporis natalis (P.M. McCarthy) S.H. Jiang, Lücking \& Sérus., Fungal Diversity 10.1007/s13225-020-00462-6: 129 (2020)

*Dichoporis nipponica (H. Harada) S.H. Jiang, Lücking \& Sérus., Fungal Diversity 10.1007/s13225-020-00462-6: 129 (2020)

*Dichoporis occulta (P.M. McCarthy \& Malcolm) S.H. Jiang, Lücking \& Sérus., Fungal Diversity 10.1007/s13225-02000462-6: 129 (2020)

*Dichoporis phaea (Ach.) S.H. Jiang, Lücking \& Sérus., Fungal Diversity 10.1007/s13225020-00462-6: 129 (2020)

*Dichoporis subprospersella (Vain.) S.H. Jiang, Lücking \& Sérus., Fungal Diversity 10.1007/s13225-020-00462-6: 129 (2020)

*Dichoporis subsimplicans (Nyl.) S.H. Jiang, Lücking \& Sérus., Fungal Diversity 10.1007/s13225-020-00462-6: 129 (2020)

*Dichoporis taylorii (Carroll) S.H. Jiang, Lücking \& Sérus., Fungal Diversity 10.1007/s13225-020-00462-6: 129 (2020)

*Dichoporis tenuis (R.C. Harris) S.H. Jiang, Lücking \& Sérus., Fungal Diversity 10.1007/s13225-020-00462-6: 129 (2020)

*Dichoporis viridiseda (Nyl.) S.H. Jiang, Lücking \& Sérus., Fungal Diversity 10.1007/s13225-020-00462-6: 129 (2020)

*Dichoporis wilsonii (Riddle) S.H. Jiang,
Lücking \& Sérus., Fungal Diversity 10.1007/s13225-020-00462-6: 129 (2020)

*Dichoporis ziziphi (A. Massal.) S.H. Jiang, Lücking \& Sérus., Fungal Diversity 10.1007/s13225-020-00462-6: 130 (2020)

*Didymella combreti (Crous) L.W. Hou, L. Cai \& Crous, Stud. Mycol. 96: 353 (2020)

*Didymella prosopidis (Crous \& A.R. Wood) L.W. Hou, L. Cai \& Crous, Stud. Mycol. 96: 359 (2020)

*Dimorphoma saxea (Aveskamp, Gruyter \& Verkley) L.W. Hou, L. Cai \& Crous, Stud. Mycol. 96: 388 (2020)

*Dinemasporium pingue (Nag Raj) W.J. Li \& K.D. Hyde, Fungal Diversity 100: 495 (2020)

*Dinemasporium setulosum (B. Sutton) W. J. Li \& K. D. Hyde, Fungal Diversity 100: 495 (2020)

Diorygma occultum (Adaw. \& Makhija) Pushpi Singh \& Kr. P. Singh, Lichenologist 52 (3): 251 (2020)

Diplocarpon coronariae (Ellis \& Davis) Wöhner \& Rossman, Fungal Systematics and Evolution 6: 186 (2020)

Dirinaria endocrocea (D.D. Awasthi) Kalb, Schumm \& Elix, Australas. Lichenol. 86: 7 (2020)

Dissophora globulifera (O. Rostr.) Vandepol $\&$ Bonito, Fungal Diversity 10.1007/s13225020-00455-5: 13 (2020)

Dothiorella alpina (Y. Zhang ter \& Ming Zhang) Phookamsak \& Hyde, Asian Journal of Mycology 3 (1): 168 (2020)

*Ectodidymella nigrificans (P. Karst.) L.W. Hou, L. Cai \& Crous, Stud. Mycol. 96: 340 (2020)

*Ectophoma insulana (Mont.) L.W. Hou, L. Cai \& Crous, Stud. Mycol. 96: 335 (2020)

Elaiopezia boudieri (Cooke) Van Vooren, Ascomycete.org 12 (4): 185 (2020)

Elaiopezia luteola (Velen.) Van Vooren, Ascomycete.org 12 (4): 185 (2020)

Elaiopezia obtusapiculata (J. Moravec) Van 
Vooren, Cahiers de la FMBDS 7: 10 (2020)

Elaiopezia polaripapulata (J. Moravec) Van Vooren, Ascomycete.org 12 (4): 185 (2020)

*Ellismarsporium varium (Alves-Barb., Malosso \& R.F. Castañeda) K. Zhang, Alves-Barb., Malosso \&. R.F. Castañeda, Mycotaxon 135 (2): 448 (2020)

Emmanuelia americana (Vain.) Lücking, Moncada \& Gumboski, Plant and Fungal Systematics 65 (1): 83 (2020)

Emmanuelia conformis (Vain.) Lücking, Moncada \& Ant. Simon, Plant and Fungal Systematics 65 (1): 85 (2020)

Emmanuelia cuprea (Müll. Arg.) Lücking, Moncada \& Ant. Simon, Plant and Fungal Systematics 65 (1): 85 (2020)

Emmanuelia elaeodes (Malme) Lücking, Spielmann \& S.M. Martins, Plant and Fungal Systematics 65 (1): 85 (2020)

Emmanuelia erosa (Eschw.) Lücking, M. Cáceres \& Ant. Simon, Plant and Fungal Systematics 65 (1): 86 (2020)

Emmanuelia excisa (Müll. Arg.) Lücking, Moncada \& Ant. Simon, Plant and Fungal Systematics 65 (1): 86 (2020)

Emmanuelia lobulifera (B.J. Moore) Ant. Simon \& Goffinet, Plant and Fungal Systematics 65 (1): 86 (2020)

Emmanuelia ornata (Malme) Lücking, Moncada \& Bungartz, Plant and Fungal Systematics 65 (1): 88 (2020)

Emmanuelia patinifera (Taylor) Lücking, M. Cáceres \& Ant. Simon, Plant and Fungal Systematics 65 (1): 88 (2020)

Emmanuelia pseudolivacea (Zahlbr.) Lücking, Moncada \& Ant. Simon, Plant and Fungal Systematics 65 (1): 89 (2020)

Emmanuelia ravenelii (Tuck.) Ant. Simon \& Goffinet, Plant and Fungal Systematics 65 (1): 89 (2020)

Emmanuelia tenuis (Vain.) Lücking, Moncada \& Gumboski, Plant and Fungal Systematics 65 (1): 91 (2020)
Endoconidioma carpetanum (Bills, Peláes \& Ruibal) Crous, Persoonia 45: 301 (2020)

Endoconidioma leucospermi (Crous \& Denman) Crous, Persoonia 45: 301 (2020)

Entoloma albertinae (Karstedt \& Capelari) Blanco-Dios, Index Fungorum 458: 1 (2020)

Entoloma atropapillatum (Karstedt \& Capelari) Blanco-Dios, Index Fungorum 458: 1 (2020)

Entoloma coeruleomagnum var. cyanatrum (Eyssart. \& Buyck) Blanco-Dios, Yesca 32: 75 (2020)

Entoloma elaphines (Berk. \& Broome) Blanco-Dios, Yesca 32: 75 (2020)

Entoloma greigense (Peck) Blanco-Dios, Yesca 32: 75 (2020)

Entoloma pallidosalmoneum (Karstedt \& Capelari) Blanco-Dios, Index Fungorum 458: 1 (2020)

Entoloma porphyrocephalum (Noordel. \& Wölfel) Noordel., Brandrud \& Dima, Agarica 39: 46 (2020)

Entoloma tricholomatoideum (Karstedt \& Capelari) Blanco-Dios, Index Fungorum 458: 1 (2020)

Entomortierella beljakovae (Milko) Vandepol \& Bonito, Fungal Diversity 10.1007/s13225020-00455-5: 15 (2020)

Entomortierella chlamydospora (Chesters) Vandepol \& Bonito, Fungal Diversity 10.1007/s13225-020-00455-5: 15 (2020)

Entomortierella echinosphaera (PlaatsNiterink) Vandepol \& Bonito, Fungal Diversity 10.1007/s13225-020-00455-5: 15 (2020)

Entomortierella lignicola (G.W. Martin) Vandepol \& Bonito, Fungal Diversity 10.1007/s13225-020-00455-5: 16 (2020)

Entomortierella parvispora (Linnem.) Vandepol \& Bonito, Fungal Diversity 10.1007/s13225-020-00455-5: 16 (2020)

*Epicoccum tobaicum (Szilvinyi) L.W. Hou, L. Cai \& Crous, Stud. Mycol. 96: 348 (2020) 
Erichansenia cryodesertorum (Garrido-Ben., Søchting \& Pérez-Ortega) S.Y. Kondr., Kärnefelt \& A. Thell, Acta bot. hung. 62 (12): 121 (2020)

Erichansenia epithallina (Lynge) S.Y. Kondr., Kärnefelt \& A. Thell, Acta bot. hung. 62 (12): 120 (2020)

Erichansenia sauronii (Søchting \& Øvstedal) S.Y. Kondr., Kärnefelt \& A. Thell, Acta bot. hung. 62 (1-2): 121 (2020)

*Ernakulamia xishuangbannaensis (Tibpromma \& K.D. Hyde) W. Dong, H. Zhang \& K.D. Hyde, Fungal Diversity 10.1007/s13225-020-00463-5: 179 (2020)

Erysiphe ehrenbergii (Lév.) U. Braun, M. Bradshaw \& S. Takam., Fungal Systematics and Evolution 7: 54 (2020)

Euantennaria atra (Bat.) Sugiy. \& Hosoya, Mycoscience 61 (6): 355 (2020)

Euantennaria cubensis (S. Hughes \& G.R.W. Arnold) Sugiy. \& Hosoya, Mycoscience 61 (6): 355 (2020)

Euantennaria dingleyae (S. Hughes) Sugiy. \& Hosoya, Mycoscience 61 (6): 355 (2020)

Euantennaria fisherae (S. Hughes) Sugiy. \& Hosoya, Mycoscience 61 (6): 355 (2020)

Euantennaria fraserae (S. Hughes) Sugiy. \& Hosoya, Mycoscience 61 (6): 355 (2020)

Euantennaria katumotoi (Sugiy. \& Hosoya) Sugiy. \& Hosoya, Mycoscience 61 (6): 356 (2020)

Euantennaria lumbricoidea (Dearn.) Sugiy. \& Hosoya, Mycoscience 61 (6): 356 (2020)

Euantennaria pinophila (Nees) Sugiy. \& Hosoya, Mycoscience 61 (6): 356 (2020)

Euantennaria shawiae (S. Hughes) Sugiy. \& Hosoya, Mycoscience 61 (6): 356 (2020)

Euantennaria triseptata (S. Hughes) Sugiy. \& Hosoya, Mycoscience 61 (6): 357 (2020)

Eupezizella aureliella (Nyl.) T. Kosonen, Huhtinen \& K. Hansen, Persoonia 46: 49 (2020)

Eupezizella britannica (Huhtinen) T. Kosonen,
Huhtinen \& K. Hansen, Persoonia 46: 49 (2020)

Eupezizella nipponica (Huhtinen) T. Kosonen, Huhtinen \& K. Hansen, Persoonia 46: 49 (2020)

Eupezizella roseoguttata (Huhtinen) $\mathrm{T}$. Kosonen, Huhtinen \& K. Hansen, Persoonia 46: 49 (2020)

Evansstolkia leycettana (H.C. Evans \& Stolk) Houbraken, Frisvad \& Samson, Stud. Mycol. 95: 89 (2020)

*Fagicola fagi (Crous \& de Hoog) Crous, M. Shen \& Y. Zhang ter, Stud. Mycol. 96: 217 (2020)

Fasciodontia bugellensis (Ces.) Yurchenko, Riebesehl \& Langer, Mycol. Progr. 19 (2): 178 (2020)

Fauriea mandshuriaensis (S.Y. Kondr., Lőkös \& Hur) S.Y. Kondr. \& Yoshik. Yamam., Acta bot. hung. 62 (1-2): 121 (2020)

Fauriea trassii (Galanina \& S.Y. Kondr.) S.Y. Kondr. \& Yoshik. Yamam., Acta bot. hung. 62 (1-2): 121 (2020)

Fibricium subodoratum (P. Karst. ex Bourdot \& Galzin) Spirin, Syn. Fung. 40: 115 (2020) Fibropilus fumosifolius (Hesler) Largent, Mycotaxon 135 (4): 793 (2020)

Fissurina leucocarpoides (Nyl.) Pushpi Singh \& Kr.P. Singh, Lichenologist 52 (3): 252 (2020)

Fissurina parvicarpa (Makhija \& Adaw.) Pushpi Singh \& Kr. P. Singh, Lichenologist 52 (3): 253 (2020)

*Flagellostrigula laureriformis (Aptroot \& Lücking) Lücking, S.H. Jiang \& Sérus., Fungal Diversity 10.1007/s13225-02000462-6: 131 (2020)

*Flammula abieticola (A.H. Sm. \& Hesler) E.J. Tian \& Matheny, Mycologia 10.1080/00275514.2020.1816067: 14 (2020) *Flavocillium acerosum (Zare \& W. Gams) H. Yu, Y.B. Wang, Y. Wang \& Zhu L. Yang, Fungal Diversity 103: 20 (2020) 
*Flavocillium primulinum (Kaifuchi, Nonaka \& Masuma) H. Yu, Y.B. Wang, Y. Wang \& Zhu L. Yang, Fungal Diversity 103: 21 (2020)

*Flavocillium subprimulinum (S.K. Huang \& K.D. Hyde) H. Yu, Y.B. Wang, Y. Wang \& Zhu L. Yang, Fungal Diversity 103: 22 (2020)

Fomitiporella coruscans (Murrill) SalvadorMontoya \& Popoff, Pl. Syst. Evol. 306 (no. 34): 16 (2020)

Fomitiporia elegans (J.E. Wright \& Blumenf.) Alves-Silva, Robledo \& Drechsler-Santos, Mycol. Progr. 19 (8): 784 (2020)

*Foraminispora austrosinensis (J.D. Zhao \& L.W. Hsu) Y.F. Sun \& B.K. Cui, Persoonia 44: 215 (2020)

*Foraminispora concentrica (J. Song, Xiao L. He \& B.K. Cui) Y.F. Sun \& B.K. Cui, Persoonia 44: 218 (2020)

*Foraminispora yunnanensis (J.D. Zhao \& X.Q. Zhang) Y.F. Sun \& B.K. Cui, Persoonia 44: 220 (2020)

Fouragea alba (Lücking) Ertz, Phytotaxa 472 (2): 189 (2020)

Fouragea heliabravoa (Herrera-Camp. \& Lücking) Ertz, Phytotaxa 472 (2): 189 (2020)

Fouragea tuxtlensis (Herrera-Camp. \& Lücking) Ertz, Phytotaxa 472 (2): 189 (2020)

Fouragea vegae (R. Sant.) Ertz, Phytotaxa 472 (2): 189 (2020)

*Fraxinicola fraxini (Aderh.) Crous, M. Shen \& Y. Zhang ter, Stud. Mycol. 96: 219 (2020)

*Fraxinicola orni (M. Ibrahim, M. Schlegel \& T.N. Sieber) Crous, M. Shen \& Y. Zhang ter, Stud. Mycol. 96: 221 (2020)

Fulgogasparrea awasthii (Y. Joshi \& Upreti) S.Y. Kondr., Upreti \& A. Thell, Acta bot. hung. 62 (3-4): 349 (2020)

Fusarium acutisporum (Sand.-Den. \& Crous) O'Donnell, Geiser, Kasson \& T. Aoki, Index Fungorum 440: 1 (2020)

Fusarium amplum (Sand.-Den. \& Crous)
O'Donnell, Geiser, Kasson \& T. Aoki, Index Fungorum 440: 1 (2020)

Fusarium bataticola (Sand.-Den. \& Crous) O'Donnell, Geiser, Kasson \& T. Aoki, Index Fungorum 440: 1 (2020)

Fusarium bomiense (Z.Q. Zeng \& W.Y. Zhuang) O'Donnell, Geiser, Kasson \& T. Aoki, Index Fungorum 440: 1 (2020)

Fusarium borneense (Petr.) O'Donnell, Geiser, Kasson \& T. Aoki, Index Fungorum 440: 1 (2020)

Fusarium breve (Sand.-Den. \& Crous) O'Donnell, Geiser, Kasson \& T. Aoki, Index Fungorum 440: 1 (2020)

Fusarium breviconum (Wollenw.) O'Donnell, Geiser, Kasson \& T. Aoki, Index Fungorum 440: 1 (2020)

Fusarium catenatum (Sand.-Den. \& Crous) O'Donnell, Geiser \& T. Aoki, Index Fungorum 440: 1 (2020)

Fusarium crassum (Sand.-Den. \& Crous) O'Donnell, Geiser, Kasson \& T. Aoki, Index Fungorum 440: 1 (2020)

Fusarium croci (Guarnaccia, Sand.-Den. \& Crous) O'Donnell, Geiser \& T. Aoki, Index Fungorum 440: 1 (2020)

Fusarium cryptoseptatum (Sand.-Den. \& Crous) O'Donnell, Geiser, Kasson \& T. Aoki, Index Fungorum 440: 1 (2020)

Fusarium cyanescens (G.A. de Vries, de Hoog \& Bruyn) O'Donnell, Geiser \& T. Aoki, Index Fungorum 440: 2 (2020)

Fusarium diminutum (Sand.-Den. \& Crous) O'Donnell, Geiser, Kasson \& T. Aoki, Index Fungorum 440: 2 (2020)

Fusarium ferrugineum (Sand.-Den. \& Crous) O'Donnell, Geiser, Kasson \& T. Aoki, Index Fungorum 440: 2 (2020)

Fusarium hengyangense (Z.Q. Zeng \& W.Y. Zhuang) O'Donnell, Geiser, Kasson \& T. Aoki, Index Fungorum 440: 2 (2020)

Fusarium hypothenemi (Sand.-Den. \& Crous) O'Donnell, Geiser, Kasson \& T. Aoki, Index 
Fungorum 440: 2 (2020)

Fusarium kuroshium (F. Na, J.D. Carrillo \& A. Eskalen ex Sand.-Den. \& Crous) O'Donnell, Geiser, Kasson \& T. Aoki, Index Fungorum 440: 2 (2020)

Fusarium liriodendri (Sand.-Den. \& Crous) O'Donnell, Geiser, Kasson \& T. Aoki, Index Fungorum 440: 2 (2020)

Fusarium macrosporum (Sand.-Den., Guarnaccia \& Polizzi) O'Donnell, Geiser \& T. Aoki, Index Fungorum 440: 2 (2020)

Fusarium mori (Sand.-Den. \& Crous) O'Donnell, Geiser, Kasson \& T. Aoki, Index Fungorum 440: 2 (2020)

Fusarium noneumartii (Sand.-Den. \& Crous) O'Donnell, Geiser, Kasson \& T. Aoki, Index Fungorum 440: 3 (2020)

Fusarium oblongum (Sand.-Den. \& Crous) O'Donnell, Geiser, Kasson \& T. Aoki, Index Fungorum 440: 3 (2020)

Fusarium ornamentatum (M.A.F. Barbosa) O'Donnell, Geiser \& T. Aoki, Index Fungorum 440: 3 (2020)

Fusarium paraeumartii (Sand.-Den. \& Crous) O'Donnell, Geiser, Kasson \& T. Aoki, Index Fungorum 440: 3 (2020)

Fusarium parceramosum (Sand.-Den. \& Crous) O'Donnell, Geiser, Kasson \& T. Aoki, Index Fungorum 440: 3 (2020)

Fusarium perseae (Sand.-Den. \& Guarnaccia) O'Donnell, Geiser \& T. Aoki, Index Fungorum 440: 3 (2020)

Fusarium piperis (F.C. Albuq.) O'Donnell, Geiser, Kasson \& T. Aoki, Index Fungorum 440: 3 (2020)

Fusarium protoensiforme (Sand.-Den. \& Crous) O'Donnell, Geiser, Kasson \& T. Aoki, Index Fungorum 440: 3 (2020)

Fusarium pseudoradicicola (Sand.-Den. \& Crous) O'Donnell, Geiser, Kasson \& T. Aoki, Index Fungorum 440: 3 (2020)

Fusarium pseudotonkinense (Sand.-Den. \& Crous) O'Donnell, Geiser, Kasson \& T. Aoki,
Index Fungorum 440: 3 (2020)

Fusarium ramosum (Batista \& H. Maia) O'Donnell, Geiser \& T. Aoki, Index Fungorum 440: 4 (2020)

Fusarium regulare (Sand.-Den. \& Crous) O'Donnell, Geiser, Kasson \& T. Aoki, Index Fungorum 440: 4 (2020)

Fusarium rhizophorae (Dayarathne) O'Donnell, Geiser, Kasson \& T. Aoki, Index Fungorum 440: 4 (2020)

Fusarium samuelsii (Sand.-Den. \& Crous) O'Donnell, Geiser, Kasson \& T. Aoki, Index Fungorum 440: 4 (2020)

Fusarium silvicola (Sand.-Den. \& Crous) O'Donnell, Geiser, Kasson \& T. Aoki, Index Fungorum 440: 4 (2020)

Fusarium spathulatum (Sand.-Den. \& Crous) O'Donnell, Geiser, Kasson \& T. Aoki, Index Fungorum 440: 4 (2020)

Fusarium spinulosum (Pfenning) O'Donnell, Geiser, Kasson \& T. Aoki, Index Fungorum 440: 4 (2020)

Fusarium suttonianum (Sand.-Den. \& Crous) O'Donnell, Geiser \& T. Aoki, Index Fungorum 440: 4 (2020)

Fusarium tenuicristatum (S. Ueda \& Udagawa) O'Donnell, Geiser \& T. Aoki, Index Fungorum 440: 4 (2020)

Fusarium tonkinense (Bugnic.) O'Donnell, Geiser \& T. Aoki, Index Fungorum 440: 4 (2020)

*Fuscohilum rhodense (Crous \& M.J. Wingf.) Crous, M. Shen \& Y. Zhang ter, Stud. Mycol. 96: 200 (2020)

*Fuscohilum sicilianum (Koukol) Crous, M. Shen \& Y. Zhang ter, Stud. Mycol. 96: 200 (2020)

Fuscohypha kunmingensis (Phookamsak, J.F. Li \& K.D. Hyde) Jayaward., Phukhams. \& K.D. Hyde, Fungal Diversity 10.1007/s13225-020-00448-4: 176 (2020)

*Fuscoporia bambusicola (L.W. Zhou \& B.S. Jia) Q. Chen, F. Wu \& Y.C. Dai, 
Mycosphere 11 (1): 1503 (2020)

Fuscoporia licnoides (Mont.) Oliveira-Filho \& Gibertoni, Fungal Diversity 10.1007/s13225-020-00461-7: 129 (2020)

*Fuscoporia roseocinerea (Murrill) Q. Chen, F. Wu \& Y.C. Dai, Mycosphere 11 (1): 1503 (2020)

Fuscoporia scruposa (Fr.) Gibertoni \& Oliveira-Filho, Fungal Diversity 10.1007/s13225-020-00461-7: 130 (2020)

Fusicolla reyesiana (Sacc.) Forin \& Vizzini, Persoonia 45: 243 (2020)

Gabura borbonica (Magain \& Sérus.) Magain \& Sérus., Lichenologist 52 (1): 11 (2020)

Gabura fascicularis var. colensoi (C. Bab.) de Lange \& M. Ford, Index Fungorum 446: 1 (2020)

Gabura fascicularis var. microcarpa (Müll. Arg.) de Lange \& M. Ford, Index Fungorum 446: 1 (2020)

Gabura insignis (P.M. Jørg. \& Tønsberg) Magain \& Sérus., Lichenologist 52 (1): 13 (2020)

Gamsiella stylospora (Dixon-Stew.) Vandepol $\&$ Bonito, Fungal Diversity 10.1007/s13225020-00455-5: 14 (2020)

Gamsomyces longisporus (M.B. Ellis) Hern.Restr. \& Réblová, Stud. Mycol. 95: 449 (2020)

Gamsomyces stilboideus (R.F. Castañeda \& G.R.W. Arnold) Hern.-Restr. \& Réblová, Stud. Mycol. 95: 451 (2020)

*Gamszarea indonesiaca (Kurihara \& Sukarno) Z.F. Zhang \& L. Cai, Fungal Diversity 10.1007/s13225-020-00453-7: 69 (2020)

*Gamszarea kalimantanensis (Kurihara \& Sukarno) Z.F. Zhang \& L. Cai, Fungal Diversity 10.1007/s13225-020-00453-7: 72 (2020)

*Gamszarea restricta (Hubka, Kubátová, Nonaka, Čmoková \& Řehulka) Z.F. Zhang \& L. Cai, Fungal Diversity 10.1007/s13225-
020-00453-7: 72 (2020)

*Gamszarea testudinea (Hubka, Kubátová, Nonaka, Čmoková \& Řehulka) Z.F. Zhang $\&$ L. Cai, Fungal Diversity 10.1007/s13225020-00453-7: 73 (2020)

*Gamszarea wallacei (H.C. Evans) Z.F. Zhang \& L. Cai, Fungal Diversity 10.1007/s13225020-00453-7: 69 (2020)

Geohypha (Fr.) Hennebert, Mycotaxon 135 (3): 708 (2020)

Geohypha terrestris (Fr.) Hennebert, Mycotaxon 135 (3): 709 (2020)

Geoscypha ampelina (Gillet) Van Vooren \& Dougoud, Ascomycete.org 12 (4): 187 (2020)

Geoscypha tenacella (Sacc.) Van Vooren, Ascomycete.org 12 (4): 187 (2020)

Glabrocyphella culmicola (Fuckel) U. Braun \& Bensch, Schlechtendalia 37: 120 (2020)

*Glaciozyma antarctica (Fell, Statzell, I.L. Hunter \& Phaff) M. Groenew. \& Q.M. Wang, Stud. Mycol. 96: 134 (2020)

Gloeocystidiellum granulatum (Sheng $\mathrm{H}$. Wu) E. Larss. \& K.H. Larss., Syn. Fung. 40: 116 (2020)

Gloeocystidiellum permixtum (Boidin, Lanq. \& Gilles) E. Larss. \& K.H. Larss., Syn. Fung. 40: 116 (2020)

Golovinomyces ixodiae (Cunningt., Beilharz \& Pascoe) U. Braun \& L. Kiss, Frontiers in Microbiology 11 (no. 1571): 22 (2020)

*Golovinomyces latisporus (U. Braun) P.L. Qiu \& S.Y. Liu, BMC Microbiology 20 (no. 51): 11 (2020)

Graphiothecium penicillatum (Fuckel) U. Braun \& Bensch, Schlechtendalia 37: 84 (2020)

Grigorovia humatica (Mikata \& Ueda-Nishim.) Gouliamova \& Dimitrov, Comptes Rendus de l'Académie Bulgare des Sciences 73 (1): 55 (2020)

Grigorovia transvaalensis (Van der Walt) Gouliamova \& Dimitrov, Comptes Rendus de l'Académie Bulgare des Sciences 73 (1): 
55 (2020)

Grigorovia yakushimaensis (Mikata \& UedaNishim.) Gouliamova \& Dimitrov, Comptes Rendus de l'Académie Bulgare des Sciences 73 (1): 55 (2020)

Gryganskiella cystojenkinii (W. Gams \& Veenb.-Rijks) Vandepol \& Bonito, Fungal Diversity 10.1007/s13225-020-00455-5: 16 (2020)

Gryganskiella fimbricystis (W. Gams) Vandepol \& Bonito, Fungal Diversity 10.1007/s13225-020-00455-5: 16 (2020)

Guarroa acutispora (Matsush.) Heredia \& R.

F. Castañeda, Mycol. Progr. 19 (1): 23 (2020)

Guayaquilia cubensis (R.F. Castañeda \& G.R.W. Arnold) R.F. Castañeda, Magdana, D. Sosa \& Hern.-Restr., Mycotaxon 135 (3): 507 (2020)

Gyalidea halocarpa (P.M. McCarthy \& Elix) P.M. McCarthy, Australas. Lichenol. 87: 30 (2020)

Gymnosporangium raphiolepidis (Syd. \& $\mathrm{P}$. Syd.) T. Kasuya, K. Hosaka \& Kakish., Phytotaxa 460 (1): 112 (2020)

*Gymnostellatospora bhattii (Samson) Zhi.Y. Zhang, Y.F. Han \& Z.Q. Liang, Frontiers in Microbiology 11 (no. 572596): 5 (2020)

Gymnotelium speciosum (Peck) Aime \& McTaggart, Fungal Systematics and Evolution 7: 41 (2020)

Halocryptosphaeria bathurstensis (K.D. Hyde \& Rappaz) Dayarathne, Devadatha, V.V. Sarma \& K.D. Hyde, Mycosphere 11 (1): 137 (2020)

Halocryptovalsa avicenniae (Abdel-Wahab, Bahkali \& E.B.G. Jones) Dayar. \& K.D. Hyde, Index Fungorum 442: 1 (2020)

Halocryptovalsa avicenniae (Abdel-Wahab, Bahkali \& E.B.G. Jones) Dayar. \& K.D. Hyde, Cryptog. Mycol. 41 (3): 50 (2020)

Hamigera brevicompacta (H.Z. Kong) Houbraken, Frisvad \& Samson, Stud. Mycol. 95: 89 (2020)
Hansenopezia retrocurvata (K. Hansen \& Sandal) Matočec, I. Kušan \& Jadan, Fungal Diversity 10.1007/s13225-020-00461-7: 64 (2020)

Hausknechtia floriformis (Hauskn.) D. Wächt. \& A. Melzer, Mycol. Progr. 19 (11): 1234 (2020)

Helgardiomyces anguioides (Nirenberg) Crous, Fungal Systematics and Evolution 7: 83 (2020)

Helicoascotaiwania farinosa (Linder) Réblová, Hern.-Restr. \& J. Fourn., Stud. Mycol. 95: 452 (2020)

Helicobolomyces cinnabarinula (Müll. Arg.) Wijayaw. \& Ertz, Mycosphere 11 (1): 1102 (2020)

Hemileccinum brunneotomentosum (B. Ortiz) Nitson \& J.L. Frank, Index Fungorum 443: 1 (2020)

Hemileccinum hortonii (A.H. Sm. \& Thiers) M. Kuo \& B. Ortiz, Mycologia 112 (1): 205 (2020)

Hemileccinum rubropunctum (Peck) Halling \& B. Ortiz, Mycologia 112 (1): 205 (2020)

*Heterosphaeria hendersonioides (Fautrey \& Lambotte) W.J. Li \& K.D. Hyde, Fungal Diversity 100: 541 (2020)

*Heterosphaeria umbilicata (Pers.) W.J. Li \& K.D. Hyde, Fungal Diversity 100: 542 (2020)

Hirticrusta subradiata (Lloyd) Matozaki, T. Hatt. \& Sotome, Mycoscience 61 (5): 245 (2020)

Hispidopannaria dasyclada (Zahlbr.) Elvebakk, Mycol. Progr. 19 (11): 1357 (2020)

*Hispidopannaria hispidula (Nyl.) Elvebakk, S.G. Hong \& C.H. Park, Mycol. Progr. 19 (11): 1357 (2020)

Homophron submaculatum (G.F. Atk.) Voto, Rivista Micologica Romana, Boll. AMER 110 (2): 89 (2020)

Hormodochis eucalypti (Crous) Crous, Persoonia 44: 319 (2020)

Hortiboletus coccyginus (Theirs) C.F. 
Schwarz, N. Siegel \& J.L. Frank, Fungal Systematics and Evolution 6: 284 (2020)

Huneckia wrightii (Tuck.) Arup, Søchting \& Bungartz, Plant and Fungal Systematics 65 (2): 530 (2020)

Hyalorbilia anonyma (Rehm) Baral, Monogr. Orbiliomycetes: 429 (2020)

Hyalorbilia citrina (A.L. Sm.) Baral, Monogr. Orbiliomycetes: 359 (2020)

Hyalorbilia fusarina (Burghouts \& W. Gams) E. Weber \& Baral, Monogr. Orbiliomycetes: 442 (2020)

Hyalorbilia fusarioides (D.C. Harris) E. Weber \& Baral, Monogr. Orbiliomycetes: 443 (2020)

Hyalorbilia leguminacea (J. Chen, L.L. Xu, B. Liu \& Xing Z. Liu) E. Weber \& Baral, Monogr. Orbiliomycetes: 442 (2020)

Hyalorbilia lignatilis (M.H. Mo \& K.Q. Zhang) E. Weber \& Baral, Monogr. Orbiliomycetes: 358 (2020)

Hyalorbilia spermatophaga (Drechsler) E. Weber \& Baral, Monogr. Orbiliomycetes: 443 (2020)

Hyaloscypha desmidiacea (Voglmayr) K. Yamag., Chuaseehar. \& Nakagiri, Mycoscience 61 (6): 278 (2020)

Hyaloscypha japonensis (Tubaki) K. Yamag., Huhtinen, Hosoya, Chuaseehar. \& Nakagiri, Mycoscience 61 (6): 278 (2020)

Hyaloscypha leucostigma (Fuckel) Baral, Monogr. Orbiliomycetes: 1676 (2020)

Hyaloscypha macrospora (Matsush.) K. Yamag., Chuaseehar. \& Nakagiri, Mycoscience 61 (6): 279 (2020)

Hyaloscypha spinulosa (Beverw.) K. Yamag., Chuaseehar. \& Nakagiri, Mycoscience 61 (6): 278 (2020)

Hydropunctaria symbalana (Nyl.) Cl. Roux, Catalogue des lichens et champignons lichénicoles de France métropolitaine. 3e édition revue et augmentée 1: 1286 (2020)

Hydropus rugosodiscus (Peck) Matheny,
Southeastern Naturalist 19 (3): 452 (2020)

Hygroaster nodulisporus (Dennis) Ralaiv., Niskanen \& Liimat., Mycol. Progr. 19: 1298 (2020)

Hymenopleella schefflerae (V.P. Abreu, A.W.C. Rosado \& O.L. Pereira) Samarak., Maharachch. \& K.D. Hyde, Journal of Fungi 6 (3): 23 (2020)

Hymenoscyphus tengii (W.Y. Zhuang) Baral, Index Fungorum 428: 1 (2020)

Imleria pallida (Frost) A. Farid, A.R. Franck, \& J. Bolin, Mycologia 112 (2): 431 (2020)

Imleria spadiceomaculans ( $\mathrm{H}$. Engel \& W. Härtl) C. Hahn, Die Pilze Deutschlands: 315 (2020)

Induratia alba (Worapong, Strobel \& W.M. Hess) Samarak., Thongbai, K.D. Hyde \& M. Stadler, Fungal Diversity 101: 193 (2020)

Induratia camphorae (Meshram, N. Kapoor, G. Chopra \& S. Saxena) Samarak., Thongbai, K.D. Hyde \& M. Stadler, Fungal Diversity 101: 193 (2020)

Induratia cinnamomi (Suwannar., Bussaban, K.D. Hyde \& Lumyong) Samarak., Thongbai, K.D. Hyde \& M. Stadler, Fungal Diversity 101: 193 (2020)

Induratia coffeana (A.A.M. Gomes, D.B. Pinho \& O.L. Pereira) Samarak., Thongbai, K.D. Hyde \& M. Stadler, Fungal Diversity 101: 193 (2020)

Induratia crispans (A.M. Mitch., Strobel, W.M. Hess, Pérez-Vargas \& Ezra) Samarak., Thongbai, K.D. Hyde \& M. Stadler, Fungal Diversity 101: 193 (2020)

Induratia equiseti (Suwannar. \& Lumyong) Samarak., Thongbai, K.D. Hyde \& M. Stadler, Fungal Diversity 101: 193 (2020)

Induratia ghoomensis (Meshram, M. Gupta \& S. Saxena) Samarak., Thongbai, K.D. Hyde \& M. Stadler, Fungal Diversity 101: 193 (2020)

Induratia indica (Meshram, M. Gupta \& S. Saxena) Samarak., Thongbai, K.D. Hyde \& 
M. Stadler, Fungal Diversity 101: 193 (2020)

Induratia musae (Suwannar. \& Lumyong) Samarak., Thongbai, K.D. Hyde \& M. Stadler, Fungal Diversity 101: 193 (2020)

Induratia oryzae (Suwannar. \& Lumyong) Samarak., Thongbai, K.D. Hyde \& M. Stadler, Fungal Diversity 101: 194 (2020)

Induratia rosea (Worapong, Strobel \& W.M. Hess) Samarak., Thongbai, K.D. Hyde \& M. Stadler, Fungal Diversity 101: 193 (2020)

Induratia strobelii (Meshram, S. Saxena \& N. Kapoor) Samarak., Thongbai, K.D. Hyde \& M. Stadler, Fungal Diversity 101: 194 (2020)

Induratia suthepensis (Suwannar. \& Lumyong) Samarak., Thongbai, K.D. Hyde \& M. Stadler, Fungal Diversity 101: 194 (2020)

Induratia suturae (Kudalkar, Strobel \& Riy.Ul-Hass.) Samarak., Thongbai, K.D. Hyde \& M. Stadler, Fungal Diversity 101: 194 (2020)

Induratia vitigena (Daisy, Strobel, Ezra \& W.M. Hess) Samarak., Thongbai, K.D. Hyde \& M. Stadler, Fungal Diversity 101: 196 (2020)

Induratia yucatanensis (M.C. González, A.L. Anaya, Glenn \& Hanlin) Samarak., Thongbai, K.D. Hyde \& M. Stadler, Fungal Diversity 101: 196 (2020)

Inocybe helobia (Kuyper) Bandini, B. Oertel \& U. Eberh., Mycol. bavarica 20: 19 (2020)

Inosperma vinaceobrunneum (Matheny, Ovrebo \& Kudzma) Haelew., Index Fungorum 436: 1 (2020)

Ionopezia gerardii (Cooke) Van Vooren, Ascomycete.org 12 (4): 188 (2020)

Ionopezia ionella (Quél.) Van Vooren, Ascomycete.org 12 (4): 188 (2020)

Italiofungus phillyreae (Thüm.) Crous, Fungal Systematics and Evolution 6: 194 (2020)

Jennwenomyces navicularis (R.F. Castañeda \& Heredia) Goh \& C.H. Kuo, Mycol. Progr. 19 (9): 874 (2020)

Jocatoa agminalis (Nyl.) Lücking, Herrera-
Camp. \& R. Miranda, Bryologist 123 (2): 138 (2020)

Jugulospora antarctica (Stchigel \& Guarro) Y. Marín \& Stchigel, Microorganisms 8 (9, no. 1430): 26 (2020)

Jugulospora carbonaria (W. Phillips \& Plowr.) Y. Marín, Stchigel, Guarro \& A.N. Mill., Microorganisms 8 (9, no. 1430): 26 (2020)

Jugulospora vestita (Udagawa \& Y. Horie) Y. Marín \& Stchigel, Microorganisms 8 (9, no. 1430): 27 (2020)

Kalmanago boliviana (M. Piepenbr.) T. Denchev, Denchev, Kemler \& Begerow, Mycobiota 10: 32 (2020)

Kalmanago combensis (Vánky) T. Denchev, Denchev, Kemler \& Begerow, Mycobiota 10: 32 (2020)

Kalmanago commelinae (Kom.) Denchev, T. Denchev, Kemler \& Begerow, Mycobiota 10: 28 (2020)

Kalmanago tinantiae (J.C. Lindq.) T. Denchev, Denchev, Kemler \& Begerow, Mycobiota 10: 32 (2020)

Kaseifertia cubensis (R.F. Castañeda \& G.R.W. Arnold) Réblová, Hern.-Restr. \& J. Fourn., Stud. Mycol. 95: 454 (2020)

Keithomyces acicularis (H. Iwasaki, Tokiwa \& Nonaka) Samson, Luangsa-ard \& Houbraken, Stud. Mycol. 95: 191 (2020)

Keithomyces carneus (Duché \& R. Heim) Samson, Luangsa-ard \& Houbraken, Stud. Mycol. 95: 189 (2020)

Keithomyces neogunnii (T.C. Wen \& K.D. Hyde) ) Luangsa-ard, Thanakitp. \& Samson, Stud. Mycol. 95: 191 (2020)

Kiliophora aseptata (Pratibha, Raghukumar \& Bhat) J.S. Monteiro, Cantillo \& Gusmão, Nova Hedwigia 110: 177 (2020)

Knightiellastrum eucalypti (Kantvilas) L. Ludw. \& Kantvilas, Lichenologist 52 (3): 218 (2020)

*Koorchaloma krabiensis (Tibpromma \& K.D. Hyde) W.J. Li \& K.D. Hyde, Fungal 
Diversity 100: 559 (2020)

*Kuehneromyces obscurus (A.H. Sm. \& Hesler) E.J. Tian \& Matheny, Mycologia 10.1080/00275514.2020.1816067: 16 (2020)

Lachnellula populina (Seaver) Pärtel \& Baral, Index Fungorum 454: 2 (2020)

Lachnellula succina (Sacc.) Polhorský \& Baral, Index Fungorum 454: 3 (2020)

Lacrima aphanotripta (Nyl.) Bungartz, Søchting \& Arup, Plant and Fungal Systematics 65 (2): 534 (2020)

Lacrima epiphora (Taylor) Bungartz, Søchting \& Arup, Plant and Fungal Systematics 65 (2): 534 (2020)

Lacrima sonorae (Wetmore) Søchting, Arup \& Bungartz, Plant and Fungal Systematics 65 (2): 540 (2020)

Lactifluus coccolobae (O.K. Mill. \& Lodge) Delgat, Persoonia 44: 287 (2020)

Lactifluus fuscomarginatus (Montoya, Bandala \& I. Haug) Delgat, Persoonia 44: 286 (2020)

Lactifluus hometii (Gillet) C. Hahn, Die Pilze Deutschlands: 418 (2020)

Lactifluus pegleri (Pacioni \& Lalli) Delgat, Persoonia 44: 295 (2020)

Lactifluus sect. Panuoidei (Singer) Delgat, Persoonia 44: 292 (2020)

Laetinaevia diaphana (Rehm) Baral, Monogr. Orbiliomycetes: 1669 (2020)

Laetinaevia epithallina (W. Phillips \& Plowr.) Baral, Index Fungorum 454: 2 (2020)

Laetinaevia raripila (Höhn.) Baral \& Helleman, Monogr. Orbiliomycetes: 1670 (2020)

Lasiobelonium pseudocorticale (Svrček) Baral, Index Fungorum 428: 1 (2020)

Lasionectria albofimbriata (Penz. \& Sacc.) Forin \& Vizzini, Persoonia 45: 232 (2020)

Leccinellum aberrans (J. Blum) C. Hahn, Mycol. bavarica 20: 142 (2020)

Leccinellum pseudoscabrum f. pseudocarpini (J. Blum) C. Hahn, Mycol. bavarica 20: 145
(2020)

Leccinellum pseudoscabrum var. brunneobadium (J. Blum) C. Hahn, Mycol. bavarica 20: 145 (2020)

Leccinellum rugosiceps (Peck) C. Hahn, Mycol. bavarica 20: 146 (2020)

Leccinellum tlemcenense (Maire) C. Hahn, Mycol. bavarica 20: 138 (2020)

Leccinum longicurvipes (Snell \& A.H. Sm.) M. Kuo \& B. Ortiz, Mycologia 112 (1): 206 (2020)

Leccinum pachyderme (Zeller \& C.W. Dodge) M. Kuo \& B. Ortiz, Mycologia 112 (1): 207 (2020)

Legaliana alaskana (E.K. Cash) Van Vooren, Ascomycete.org 12 (4): 188 (2020)

Legaliana badia (Pers.) Van Vooren, Ascomycete.org 12 (4): 188 (2020)

Legaliana badiofuscoides (Donadini) Van Vooren, Ascomycete.org 12 (4): 188 (2020)

Legaliana limnaea (Maas Geest.) Van Vooren, Ascomycete.org 12 (4): 188 (2020)

Legaliana muscicola (Donadini) Van Vooren, Ascomycete.org 12 (4): 188 (2020)

Lendemeriella borealis (Vain.) S.Y. Kondr., Acta bot. hung. 62 (1-2): 121 (2020)

Lendemeriella dakotensis (Wetmore) S.Y. Kondr., Acta bot. hung. 62 (1-2): 121 (2020)

Lendemeriella exsecuta (Nyl.) S.Y. Kondr., Acta bot. hung. 62 (1-2): 121 (2020)

Lendemeriella lucifuga (G. Thor) S.Y. Kondr., Acta bot. hung. 62 (1-2): 121 (2020)

Lendemeriella nivalis (Körb.) S.Y. Kondr., Acta bot. hung. 62 (1-2): 121 (2020)

Lendemeriella reptans (Lendemer \& B.P. Hodk.) S.Y. Kondr., Acta bot. hung. 62 (12): 121 (2020)

Lendemeriella sorocarpa (Vain.) S.Y. Kondr., Acta bot. hung. 62 (1-2): 121 (2020)

Lendemeriella tornoensis (H. Magn.) S.Y. Kondr., Acta bot. hung. 62 (1-2): 122 (2020) Lepra leeuwenii (Zahlbr.) A.W. Archer \& Elix, Australas. Lichenol. 86: 15 (2020) 
Lepra subvelata (G. Merrill) T. Sprib., Lichenologist 52 (2): 132 (2020)

Leptobacillium chinense (F. Liu \& L. Cai) Okane, Nonaka, Kurihara, J.P. Abe \& Yamaoka, Mycoscience 61 (4): 169 (2020)

Leptobacillium coffeanum (A.A.M. Gomes \& O.L. Pereira) Okane, Nonaka, Kurihara, J.P. Abe \& Yamaoka, Mycoscience 61 (4): 169 (2020)

Leptodontidium diaphanulum (Cooke) Baral, Monogr. Orbiliomycetes: 1669 (2020)

Leptonia cyanea (Sacc.) Largent, Mycotaxon 135 (4): 794 (2020)

Leptoxyphium fumago (Woron.) Crous, Fungal Systematics and Evolution 6: 198 (2020)

Leucoinocybe auricoma (Har. Takah.) Matheny, Southeastern Naturalist 19 (3): 456 (2020)

*Leucosporidium creatinivorum (Golubev) M. Groenew. \& Q.M. Wang, Stud. Mycol. 96: 134 (2020)

*Leucosporidium fragarium (J.A. Barnett \& Buhagiar) M. Groenew. \& Q.M. Wang, Stud. Mycol. 96: 134 (2020)

*Leucosporidium intermedium (Nakase \& M. Suzuki) M. Groenew. \& Q.M. Wang, Stud. Mycol. 96: 134 (2020)

*Leucosporidium muscorum (Di Menna) M. Groenew. \& Q.M. Wang, Stud. Mycol. 96: 134 (2020)

*Leucosporidium yakuticum (Golubev) M. Groenew. \& Q.M. Wang, Stud. Mycol. 96: 134 (2020)

Lichenopeltella inconspicua (J. Lahm) Cl. Roux, Catalogue des lichens et champignons lichénicoles de France métropolitaine. 3e édition revue et augmentée 1: 1286 (2020)

Liladisca acicola (Fuckel) Baral, Monogr. Orbiliomycetes: 259 (2020)

Linnemannia acrotona (W. Gams) Vandepol \& Bonito, Fungal Diversity 10.1007/s13225020-00455-5: 16 (2020)
Linnemannia amoeboidea (W. Gams) Vandepol \& Bonito, Fungal Diversity 10.1007/s13225-020-00455-5: 16 (2020)

Linnemannia camargensis (W. Gams \& R. Moreau) Vandepol \& Bonito, Fungal Diversity 10.1007/s13225-020-00455-5: 16 (2020)

Linnemannia elongata (Linnem.) Vandepol \& Bonito, Fungal Diversity 10.1007/s13225020-00455-5: 17 (2020)

Linnemannia exigua (Linnem.) Vandepol \& Bonito, Fungal Diversity 10.1007/s13225020-00455-5: 17 (2020)

Linnemannia gamsii (Milko) Vandepol \& Bonito, Fungal Diversity 10.1007/s13225020-00455-5: 17 (2020)

Linnemannia hyalina (Harz) Vandepol \& Bonito, Fungal Diversity 10.1007/s13225020-00455-5: 17 (2020)

Linnemannia nantahalensis (C.Y. Chien) Vandepol \& Bonito, Fungal Diversity 10.1007/s13225-020-00455-5: 17 (2020)

Linnemannia schmuckeri (Linnem.) Vandepol \& Bonito, Fungal Diversity 10.1007/s13225-020-00455-5: 17 (2020)

Linnemannia sclerotiella (Milko) Vandepol \& Bonito, Fungal Diversity 10.1007/s13225020-00455-5: 17 (2020)

Linnemannia zychae (Linnem.) Vandepol \& Bonito, Fungal Diversity 10.1007/s13225020-00455-5: 17 (2020)

Linosporopsis carpini (J. Schröt.) Voglmayr \& Beenken, Mycol. Progr. 19 (3): 212 (2020)

Linosporopsis ischnotheca (Desm.) Voglmayr \& Beenken, Mycol. Progr. 19 (3): 212 (2020)

Linosporopsis magnagutiana (Sacc.) Voglmayr \& Beenken, Mycol. Progr. 19 (3): 215 (2020)

Linosporopsis ochracea (Sacc.) Voglmayr \& Beenken, Mycol. Progr. 19 (3): 217 (2020)

Lipocystis acaciae-pennatulae (Dietel) Aime \& McTaggart, Fungal Systematics and Evolution 7: 38 (2020) 
*Lobothallia semisterilis (H. Magn.) Y.Y. Zhang, MycoKeys 66: 142 (2020)

Loekoesia apostatica (Nyl.) Ertz \& Diederich, Plant and Fungal Systematics 65 (1): 42 (2020)

Loekoesia yuchiorum (Lendemer \& C.A. Morse) Arup \& Søchting, Plant and Fungal Systematics 65 (2): 570 (2020)

*Longididymella clematidis (Woudenb., Spiers \& Gruyter) L.W. Hou, L. Cai \& Crous, Stud. Mycol. 96: 339 (2020)

*Longididymella vitalbae (Briard \& Har.) L.W. Hou, L. Cai \& Crous, Stud. Mycol. 96: 340 (2020)

*Longiseptatispora meliloti (Lasch ex Rabenh.) L.W. Hou \& Crous, Fungal Systematics and Evolution 6: 199 (2020)

Lunasporangiospora chienii (P.M. Kirk) Vandepol \& Bonito, Fungal Diversity 10.1007/s13225-020-00455-5: 17 (2020)

Lunasporangiospora selenospora (W. Gams) Vandepol \& Bonito, Fungal Diversity 10.1007/s13225-020-00455-5: 18 (2020)

Lundqvistomyces karachiensis (S.I. Ahmed \& Asad) Y. Marín \& Stchigel, Microorganisms 8 (9, no. 1430): 29 (2020)

Lundqvistomyces tanzaniensis (R.S. Khan \& J.C. Krug) Y. Marín \& Stchigel, Microorganisms 8 (9, no. 1430): 29 (2020)

Lyomyces fimbriatus (Sheng $\mathrm{H} . \quad \mathrm{Wu}$ ) Riebesehl \& Yurchenko, Mycol. Progr. 19 (2): 182 (2020)

Macroconstrictolumina lyrata (R.C. Harris) Lücking, R. Miranda \& Aptroot, Fungal Diversity $\quad$ 10.1007/s13225-020-00462-6: 169 (2020)

Macroconstrictolumina majuscula (Nyl.) Lücking, R. Miranda \& Aptroot, Fungal Diversity $\quad$ 10.1007/s13225-020-00462-6: 169 (2020)

Macroconstrictolumina malaccitula (Nyl.) Lücking, R. Miranda \& Aptroot, Fungal Diversity $\quad 10.1007 / \mathrm{s} 13225-020-00462-6$ :
169 (2020)

Macrotyphula megasperma (Berthier) Olariaga, Huhtinen, Læssøe, J.H. Petersen \& K. Hansen, Stud. Mycol. 96: 175 (2020)

Macrotyphula phacorrhiza (Reichard) Olariaga, Huhtinen, Læssøe, J.H. Petersen \& K. Hansen, Stud. Mycol. 96: 175 (2020)

Magnibotryascoma rubriostiolatum (Jaklitsch \& Voglmayr) Phukhams., E.B.G. Jones \& K.D. Hyde, Fungal Diversity 10.1007/s13225-020-00448-4: 125 (2020)

Mallocybe errata (E. Horak, Matheny \& Desjardin) Haelew., MycoKeys 69: 24 (2020) Mallocybe siciliana (Brugaletta, Consiglio \& M. Marchetti) Brugaletta, Consiglio \& M. Marchetti, Index Fungorum 448: 1 (2020)

Malvipezia emileia (Cooke) Van Vooren, Ascomycete.org 12 (4): 188 (2020)

Malvipezia howsei (Roze \& Boud.) Van Vooren, Ascomycete.org 12 (4): 188 (2020)

Malvipezia lividula (W. Phillips) Van Vooren \& Dougoud, Ascomycete.org 12 (4): 188 (2020)

Malvipezia pauli (Medardi, Lantieri \& Cacialli) Van Vooren, Ascomycete.org 12 (4): 188 (2020)

Marasmiellus inoderma (Berk.) Singer ex Furneaux, Index Fungorum 453: 1 (2020)

Marasmiellus rodhallii (Desjardin \& B.A. Perry) Muh. Ali, Niazi \& Khalid, Sydowia 72: 291 (2020)

Marquandomyces marquandii (Massee) Samson, Houbraken \& Luangsa-ard, Stud. Mycol. 95: 194 (2020)

Massjukiella impolita (Arup) S.Y. Kondr., Acta bot. hung. 62 (3-4): 279 (2020)

Massjukiella pollinarioides (L. Lindblom \& D.M. Wright) S.Y. Kondr., Acta bot. hung. 62 (3-4): 279 (2020)

Massjukiella stellata (Wetmore \& Kärnefelt) S.Y. Kondr., Acta bot. hung. 62 (3-4): 279 (2020)

Massjukiella tenax (L. Lindblom) S.Y. Kondr., 
Acta bot. hung. 62 (3-4): 279 (2020)

Massjukiella tenuiloba (L. Lindblom) S.Y. Kondr., Acta bot. hung. 62 (3-4): 279 (2020)

Megalotremis elegans (R.C. Harris) Lücking \& Aptroot, Fungal Diversity 10.1007/s13225-020-00462-6: 93 (2020)

Megalotremis holopolia (Nyl.) Lücking \& Aptroot, Fungal Diversity 10.1007/s13225020-00462-6: 93 (2020)

Megalotremis immersa (Makhija \& Patw.) Lücking \& Aptroot, Fungal Diversity 10.1007/s13225-020-00462-6: 93 (2020)

Megalotremis monospora (Makhija \& Patw.) Lücking \& Aptroot, Fungal Diversity 10.1007/s13225-020-00462-6: 93 (2020)

Megaspora subpoliotera (Y. Joshi \& Upreti) S.Y. Kondr., Upreti \& A. Thell, Acta bot. hung. 62 (3-4): 382 (2020)

Melanconis marginalis subsp. italica (Senan., Camporesi \& K.D. Hyde) Jaklitsch \& Voglmayr, MycoKeys 63: 93 (2020)

*Meruliopsis albomellea (Yuan Yuan, Jia J. Chen \& X.H. Ji) C.C. Chen \& Sheng H. Wu, Mycologia 112 (1): 72 (2020)

*Meruliopsis crassitunicata (Y.C. Dai \& Sheng H. Wu) C.C. Chen \& Sheng H. Wu, Mycologia 112 (1): 72 (2020)

*Meruliopsis nanlingensis (B.K. Cui \& B.S. Jia) C.C. Chen \& Sheng H. Wu, Mycologia 112 (1): 76 (2020)

*Meruliopsis pseudocystidiata (B.S. Jia \& Y.C. Dai) C.C. Chen \& Sheng H. Wu, Mycologia 112 (1): 78 (2020)

Metacapnodium neesii (S. Hughes) Sugiy. \& Hosoya, J. Fungal Res. 18 (4): 250 (2020)

Microbotryum ducellieri (Maire) Kemler, T. Denchev, Denchev \& M. Lutz, Mycol. Progr. 19 (5): 487 (2020)

*Microconidiobolus nodosus (Sriniv. \& Thirum.) B. Huang \& Y. Nie, MycoKeys 66: 72 (2020)

*Microconidiobolus paulus (Drechsler) B. Huang \& Y. Nie, MycoKeys 66: 70 (2020)
*Microconidiobolus terrestris (Sriniv. \& Thirum.) B. Huang \& Y. Nie, MycoKeys 66: 73 (2020)

*Microthecium inferius (Udagawa \& Cain) D.W. Li, R.F. Castañeda \& N.P. Schultes, Mycotaxon 135 (6): 307 (2020)

Mimicoscypha lacrimiformis (Hosoya) $\mathrm{T}$. Kosonen, Huhtinen \& K. Hansen, Persoonia 46: 53 (2020)

Mimicoscypha paludosa (Velen.) T. Kosonen, Huhtinen \& K. Hansen, Persoonia 46: 53 (2020)

*Mirohelminthosporium bigenum (Matsush.) K. Zhang, D.W. Li \& R.F. Castañeda, Mycotaxon 135 (4): 780 (2020)

Mniaecia albida (P. Crouan \& H. Crouan) Priou \& Baral, Monogr. Orbiliomycetes: 1660 (2020)

Mollisia oblonga (C.J.K. Wang \& B. Sutton) Ingo Wagner, Florian Prell \& Baral, Index Fungorum 454: 2 (2020)

Mollisia panicicola (E. Walsh \& N. Zhang) Tanney \& Seifert, Stud. Mycol. 95: 349 (2020)

Mollisia protrusa (Berk. \& M.A. Curtis) Baral, Index Fungorum 454: 2 (2020)

Mollisia scopiformis (Kowalski \& Kehr) I. Wagner, Prell \& Baral, Index Fungorum 454 2 (2020)

Mollisia velebitica (Matocec, I. Kušan, Jadan, Tkalčec \& Mešic) Baral, Index Fungorum 428: 1 (2020)

Moniliophthora brasiliensis (Arruda, G.F. Sepúlveda, R.N.G. Mill., M.A. Ferreira \& M.S. Felipe) Niveiro, Lodge \& Aime, MycoKeys 66: 47 (2020)

Moniliophthora ticoi (Halling) Niveiro, Ramírez, Lodge \& Aime, MycoKeys 66: 48 (2020)

Murinectria antarctica (Speg.) Niranjan \& V.V. Sarma, Mycosphere 11 (1): 739 (2020) Murinectria polythalama (Berk.) Niranjan \& V.V. Sarma, Mycosphere 11 (1): 739 (2020) 
Murinectria pseudotrichia (Berk. \& M.A. Curtis) Niranjan \& V.V. Sarma, Mycosphere 11 (1): 739 (2020)

Myosporidium ladogensis (Voronin) S.R.M. Jones, H. Ahonen \& J.Taskinen, Dis. Aquat. Org. 139: 17 (2020)

Myrmecopterula moniliformis (Henn.) LealDutra, Dentinger \& G.W. Griff., IMA Fungus 11 (no. 2): 12 (2020)

Myrmecopterula nudihortorum (Dentinger) Leal-Dutra, Dentinger \& G.W. Griff., IMA Fungus 11 (no. 2): 12 (2020)

Myrmecopterula velohortorum (Dentinger) Leal-Dutra, Dentinger \& G.W. Griff., IMA Fungus 11 (no. 2): 12 (2020)

Naevia dispersa (Schrad.) Thiyagaraja., Lücking \& K.D. Hyde, Fungal Diversity 10.1007/s13225-020-00451-9: 13 (2020)

Naevia pinastri (Anzi) Thiyagaraja., Lücking \& K.D. Hyde, Fungal Diversity 10.1007/s13225-020-00451-9: 12 (2020)

Namibialina melanothrix (Laurer) Spjut \& Sérus., MycoKeys 73: 33 (2020)

Namibialina melanothrix (Laurer) Spjut \& Sérus., MycoKeys 74: 109 (2020)

Narcissea cordispora (T. Gibbs) D. Wächt. \& A. Melzer, Mycol. Progr. 19 (11): 1203 (2020)

Narcissea patouillardii (Quél.) D. Wächt. \& A. Melzer, Mycol. Progr. 19 (11): 1204 (2020)

Necromortierella dichotoma (Linnem. ex W. Gams) Vandepol \& Bonito, Fungal Diversity 10.1007/s13225-020-00455-5: 18 (2020)

Neoascotaiwania fusiformis (J. Yang, Maharachch. \& K.D. Hyde) Réblová, Hern.Restr. \& J. Fourn., Stud. Mycol. 95: 455 (2020)

Neoboletus erythropus var. junquilleus (Quél.) Blanco-Dios, Index Fungorum 449: 1 (2020) Neobrownliella cinnabarina (Ach.) S.Y. Kondr., Upreti \& A. Thell, Acta bot. hung. 62 (3-4): 362 (2020)

Neobrownliella holochracea (Nyl.) S.Y.
Kondr., Upreti \& A. Thell, Acta bot. hung. 62 (3-4): 363 (2020)

Neobuelliella poetschii (Hafellner) Hongsanan \& K.D. Hyde, Fungal Diversity 10.1007/s13225-020-00462-6: 29 (2020)

*Neoconidiobolus couchii (Sriniv. \& Thirum.) B. Huang \& Y. Nie, MycoKeys 66: 73 (2020) *Neoconidiobolus lachnodes (Drechsler) B. Huang \& Y. Nie, MycoKeys 66: 73 (2020)

*Neoconidiobolus mirabilis (Y. Nie \& B. Huang) B. Huang \& Y. Nie, MycoKeys 66: 73 (2020)

*Neoconidiobolus osmodes (Drechsler) B. Huang \& Y. Nie, MycoKeys 66: 73 (2020)

*Neoconidiobolus pachyzygosporus (Y. Nie \& B. Huang) B. Huang \& Y. Nie, MycoKeys 66: 73 (2020)

*Neoconidiobolus sinensis (Y. Nie, X.Y. Liu \& B. Huang) B. Huang \& Y. Nie, MycoKeys 66: 74 (2020)

*Neoconidiobolus stilbeus (Y. Nie \& B. Huang) B. Huang \& Y. Nie, MycoKeys 66: 74 (2020)

*Neoconidiobolus stromoideus (Sriniv. \& Thirum.) B. Huang \& Y. Nie, MycoKeys 66: 74 (2020)

*Neoconidiobolus thromboides (Drechsler) B. Huang \& Y. Nie, MycoKeys 66: 70 (2020)

*Neoconidiobolus vermicola (J.S. McCulloch) B. Huang \& Y. Nie, MycoKeys 66: 74 (2020)

*Neodermea acerina (Peck) W.J. Li \& K.D. Hyde, Fungal Diversity 100: 613 (2020)

Neoechinodiscus kozhevnikovii (Zhurb.) Molinari \& R. Sierra, Opuscula Philolichenum 19: 173 (2020)

Neoechinodiscus lesdainii (Vouaux) R. Sierra \& Molinari, Opuscula Philolichenum 19: 173 (2020)

*Neofusicladium eucalypti (Crous \& R.G. Shivas) Crous, M. Shen \& Y. Zhang ter, Stud. Mycol. 96: 203 (2020)

*Neofusicladium eucalypticola (Crous \& M.J. Wingf.) Crous, M. Shen \& Y. Zhang ter, 
Stud. Mycol. 96: 205 (2020)

*Neofusicladium regnans (Crous) Crous, M. Shen \& Y. Zhang ter, Stud. Mycol. 96: 205 (2020)

*Neohelicascus aquaticus (H. Zhang \& K.D. Hyde) W. Dong, K.D. Hyde \& H. Zhang, Fungal Diversity 10.1007/s13225-02000463-5: 148 (2020)

*Neohelicascus chiangraiensis (Z.L. Luo, J.K Liu, H.Y. Su \& K.D. Hyde) W. Dong, K.D. Hyde \& H. Zhang, Fungal Diversity 10.1007/s13225-020-00463-5: 148 (2020)

*Neohelicascus egyptiacus (Abdel-Wahab \& Abdel-Aziz) W. Dong, K.D. Hyde \& H. Zhang, Fungal Diversity 10.1007/s13225020-00463-5: 148 (2020)

*Neohelicascus elaterascus (Shearer) W. Dong, K.D. Hyde \& H. Zhang, Fungal Diversity 10.1007/s13225-020-00463-5: 149 (2020)

*Neohelicascus gallicus (Y. Zhang ter \& J. Fourn) W. Dong, K.D. Hyde \& H. Zhang, Fungal Diversity 10.1007/s13225-02000463-5: 150 (2020)

*Neohelicascus unilocularis (J. Fourn. \& Y. Zhang ter) W. Dong, K.D. Hyde \& H. Zhang, Fungal Diversity 10.1007/s13225-02000463-5: 152 (2020)

*Neohelicascus uniseptatus (J. Yang, J.K. Liu \& K.D. Hyde) W. Dong, K.D. Hyde \& H. Zhang, Fungal Diversity 10.1007/s13225020-00463-5: 153 (2020)

*Neojahnula australiensis (K.D. Hyde) W. Dong, H. Zhang \& K.D. Hyde, Fungal Diversity 10.1007/s13225-020-00463-5: 33 (2020)

Neonectria magnoliae (M.L. Lohman \& Hepting) Stauder \& Kasson, Fungal Ecology 47 (no. 100980): 7 (2020)

Neoolivea tectonae (Racib.) Aime \& McTaggart, Fungal Systematics and Evolution 7: 40 (2020)

Neophaeotheca salicorniae (Crous \& Roets)
Abdollahz. \& Crous, Stud. Mycol. 95: 392 (2020)

Neophaeotheca triangularis (de Hoog \& Beguin) Abdollahz. \& Crous, Stud. Mycol. 95: 392 (2020)

Neophysopella kraunhiae (Dietel) Aime \& McTaggart, Fungal Systematics and Evolution 7: 40 (2020)

Neophysopella wareoensis (Cummins) Y. Ono \& Okane, Mycol. Progr. 19 (9): 915 (2020)

Neosetophoma cerealis (E. Müll.) Crous, Fungal Systematics and Evolution 6: 206 (2020)

Neospermospora avenae (R. Sprague \& Aar.G. Johnson) Crous \& U. Braun, Fungal Systematics and Evolution 7: 84 (2020)

Neoxylaria juruensis (Henn.) Konta \& K.D. Hyde, Mycosphere 11 (1): 2639 (2020)

Neoxylaria queenslandica (Joanne E. Taylor, K.D. Hyde \& E.B.G. Jones) Konta \& K.D. Hyde, Mycosphere 11 (1): 2639 (2020)

*Niesslia iridicola (M.E. Barr) Crous, M. Shen \& Y. Zhang ter, Stud. Mycol. 96: 291 (2020)

*Niesslia parasitica (Ellis \& Everh.) Crous, M. Shen \& Y. Zhang ter, Stud. Mycol. 96: 293 (2020)

*Niesslia vaccinii (Ellis \& Everh.) Crous, M. Shen \& Y. Zhang ter, Stud. Mycol. 96: 293 (2020)

Nolanea papillatoides (Mešić \& Tkalčec) Largent, Mycotaxon 135 (4): 794 (2020)

Nolanea undatomarginata (Mešić \& Tkalčec) Largent, Mycotaxon 135 (4): 795 (2020)

*Nothophoma acaciae (Crous) L.W. Hou, L. Cai \& Crous, Stud. Mycol. 96: 365 (2020)

*Nothophoma eucalyptigena (Crous) L.W. Hou, L. Cai \& Crous, Stud. Mycol. 96: 367 (2020)

*Nothophoma prosopidis (Crous \& A.R. Wood) L.W. Hou, L. Cai \& Crous, Stud. Mycol. 96: 370 (2020)

Nothoseptoria caraganae (Henn.) Crous \& Bulgakov, Fungal Systematics and 
Evolution 6: 210 (2020)

Oceanoplaca caesioisidiata (Arup \& van den Boom) Arup, Plant and Fungal Systematics 65 (2): 546 (2020)

Oceanoplaca caesiosorediata (Arup \& van den Boom) Arup, Plant and Fungal Systematics 65 (2): 546 (2020)

Oceanoplaca catillarioides (Arup \& van den Boom) Arup, Plant and Fungal Systematics 65 (2): 546 (2020)

Oceanoplaca isidiosa (Vain.) Bungartz, Søchting \& Arup, Plant and Fungal Systematics 65 (2): 541 (2020)

Ocellomma rediuntum (Stizenb. ex Hasse) Kantvilas, Gueidan \& Tehler, Lichenologist 52 (3): 188 (2020)

Ochrolechia minuta (Degel.) T. Sprib., Lichenologist 52 (2): 133 (2020)

Olegblumia demissa (Flot. ex Körb.) S.Y. Kondr., Lőkös, Jung Kim, A.S. Kondr., S.O. Oh \& Hur, Index Fungorum 466: 1 (2020)

Olotia codinae (Deschuyteneer, A. Melzer \& Pérez-De-Greg.) D. Wächt. \& A. Melzer, Mycol. Progr. 19 (11): 1235 (2020)

Opeltia flavorubescens (Huds.) S.Y. Kondr. \& Hur, Acta bot. hung. 62 (3-4): 365 (2020)

Opeltiella canadensis (H. Magn.) S.Y. Kondr., Acta bot. hung. 62 (3-4): 304 (2020)

Opeltiella fibrosoides (M. Westberg \& Frödén) S.Y. Kondr., Acta bot. hung. 62 (3-4): 304 (2020)

Opeltiella fruticans (Poelt \& Oberw.) S.Y. Kondr., Acta bot. hung. 62 (3-4): 304 (2020) Opeltiella rubrisoli (D. Liu \& Hur) S.Y. Kondr., Acta bot. hung. 62 (3-4): 304 (2020) Ophiocordyceps binata (H.C. Evans \& Samson) J.P.M. Araújo, H.C. Evans \& D.P. Hughes, Mycologia 112 (6): 1154 (2020)

Orbilia bembicodes (Drechsler) E. Weber \& Baral, Monogr. Orbiliomycetes: 1626 (2020)

*Orbilia brochopaga (Drechsler) Baral, E. Weber, Bin Liu \& Z.F. Yu, Monogr. Orbiliomycetes: 1649 (2020)
Orbilia cladodes (Drechsler) E. Weber \& Baral, Monogr. Orbiliomycetes: 1520 (2020)

Orbilia clavispora (J. Chen, L.L. Xu, B. Liu \& Xing Z. Liu) Baral, E. Weber, P. Perz \& G. Marson, Monogr. Orbiliomycetes: 1501 (2020)

Orbilia disseminata (Henn.) Baral \& E. Weber, Monogr. Orbiliomycetes: 818 (2020)

Orbilia elegans (Oudem.) Baral, A. Rubner, E. Weber \& Van Ryck., Monogr. Orbiliomycetes: 1552 (2020)

*Orbilia ellipsospora (Preuss) Bin Liu, E. Weber \& Baral, Monogr. Orbiliomycetes: 1603 (2020)

Orbilia flagellispora (Raitv. \& R. Galán) Baral \& G. Marson, Monogr. Orbiliomycetes: 695 (2020)

*Orbilia javanica (Rifai \& R.C. Cooke) Bin Liu, Baral \& E. Weber, Monogr. Orbiliomycetes: 1550 (2020)

*Orbilia latispora (H.Y. Su \& X.Y. Yang) Baral, Ying Zhang \& E. Weber, Monogr. Orbiliomycetes: 1569 (2020)

*Orbilia lysipaga (Drechsler) Bin Liu, Baral \&

E. Weber, Monogr. Orbiliomycetes: 1600 (2020)

Orbilia mammillata (S.M. Dixon) Baral \& E. Weber, Monogr. Orbiliomycetes: 1594 (2020)

Orbilia multiformis (Dowsett, J. Reid \& Kalkat) E. Weber \& Baral, Monogr. Orbiliomycetes: 1547 (2020)

Orbilia oligospora (Fresen.) Baral \& E. Weber, Monogr. Orbiliomycetes: 1539 (2020)

Orbilia oxyspora (Sacc. \& Marchal) E. Weber \& Baral, Monogr. Orbiliomycetes: 1489 (2020)

Orbilia polybrocha (Drechsler) Baral, E. Weber \& Pfister, Monogr. Orbiliomycetes: 1631 (2020)

Orbilia regalis (Cooke \& Ellis) Baral, Monogr. Orbiliomycetes: 775 (2020)

Orbilia rhopalota (Drechsler) Baral \& E. 
Weber, Monogr. Orbiliomycetes: 1472 (2020)

Orbilia rosea (J. Webster \& Descals) Baral \& E. Weber, Monogr. Orbiliomycetes: 959 (2020)

Orbilia saccharifera (Berk.) Baral, Monogr. Orbiliomycetes: 821 (2020)

Orbilia scaphoides (Peach) E. Weber \& Baral, Monogr. Orbiliomycetes: 1572 (2020)

Orbilia sect. Arthrobotrys (Corda) Baral, E. Weber \& Hagedorn, Monogr. Orbiliomycetes: 1432 (2020)

Orbilia sect. Helicoon (Morgan) Baral \& E. Weber, Monogr. Orbiliomycetes: 953 (2020)

Orbilia ser. Dactylellina (M. Morelet) Baral \& E. Weber, Monogr. Orbiliomycetes: 1584 (2020)

Orbilia ser. Drechslerella (Subram.) Baral \& E. Weber, Monogr. Orbiliomycetes: 1623 (2020)

Orbilia ser. Gamsylella (M. Scholler, Hagedorn \& A. Rubner) Baral \& E. Weber, Monogr. Orbiliomycetes: 1610 (2020)

Orbilia ser. Pseudotripoconidium (Z.F. Yu \& K.Q. Zhang) Baral \& E. Weber, Monogr. Orbiliomycetes: 997 (2020)

Orbilia subgen. Habrostictis (Fuckel) Baral \& E. Weber, Monogr. Orbiliomycetes: 768 (2020)

Orbilia xinjiangensis (J. Chen, L.L. Xu, B. Liu \& Xing Z. Liu) E. Weber, Baral \& Helleman, Monogr. Orbiliomycetes: 1456 (2020)

*Orbilia yunnanensis (Z.F. Yu \& K.Q. Zhang) Bin Liu, E. Weber \& Baral, Monogr. Orbiliomycetes: 1642 (2020)

Orbilia zhongdianensis (J. Zhang \& K.Q. Zhang) Baral, E. Weber \& M. Bemmann, Monogr. Orbiliomycetes: 1470 (2020)

Osteina rhodophila (Spirin \& Zmitr.) Bernicchia \& Gorjón, Romar: 486 (2020)

Oxneriopsis bassiae (Ach.) S.Y. Kondr., Upreti \& Hur, Acta bot. hung. 62 (3-4): 367 (2020)
Pachyella pseudoampelina (Donadini) Van Vooren, Cahiers de la FMBDS 7: 33 (2020)

Pachyella salicina (Velen.) Van Vooren, Cahiers de la FMBDS 7: 33 (2020)

*Pachyma pseudococos (F. Wu, J. Song \& Y.C. Dai) F. Wu, Y.C. Dai \& V. Papp, Frontiers in Microbiology 11 (no. 590788): 7 (2020)

Paecilomyces lagunculariae (C. Ram) Houbraken, Frisvad \& Samson, Stud. Mycol. 95: 90 (2020)

Pallidogramme canarensis (Patw. \& C.R. Kulkarni) Pushpi Singh \& Kr. P. Singh, Lichenologist 52 (3): 253 (2020)

Papiliomyces liangshanensis (M. Zang, D. Liu \& R. Hu) Luangsa-ard, Samson \& Thanakitp., Stud. Mycol. 95: 240 (2020)

Papiliomyces shibinensis (T.C. Wen, J.C. Kang \& K.D. Hyde) Luangsa-ard, Samson \& Thanakitp., Stud. Mycol. 95: 241 (2020)

*Papiliotrema aspenensis (Ferreira-Paim, T.B. Ferreira, Andrade-Silva, D.J. Mora, D.J. Springer, Heitman, F.M. Fonseca, D. Matos, Melhem \& Silva-Verg.) Xin Zhan Liu, F.Y. Bai, M. Groenew. \& Boekhout, Stud. Mycol. 96: 135 (2020)

Parachaetomium carinthiacum (Sörgel) Mehrabi, Asgari \& Zare, Mycol. Progr. 19 (12): 1422 (2020)

Parachaetomium iranianum (Asgari \& Zare) Mehrabi, Asgari \& Zare, Mycol. Progr. 19 (12): 1422 (2020)

Parachaetomium truncatulum (Asgari \& Zare) Mehrabi, Asgari \& Zare, Mycol. Progr. 19 (12): 1422 (2020)

*Parafusicladium amoenum (R.F. Castañeda \& Dugan) Crous, M. Shen \& Y. Zhang ter, Stud. Mycol. 96: 206 (2020)

*Parafusicladium intermedium (Crous \& W.B. Kendr.) Crous, M. Shen \& Y. Zhang ter, Stud. Mycol. 96: 208 (2020)

*Parafusicladium paraamoenum (Crous, Jacq. Edwards \& P.W.J. Taylor) Crous, M. Shen \& Y. Zhang ter, Stud. Mycol. 96: 208 (2020) 
Paragaeumannomyces albidus (T.J. Atk., A.N. Mill. \& Huhndorf) Réblová \& A.N. Mill., MycoKeys 74: 24 (2020)

Paragaeumannomyces bombycinus (T.J. Atk., A.N. Mill. \& Huhndorf) Réblová \& A.N. Mill., MycoKeys 74: 26 (2020)

Paragaeumannomyces garethjonesii (R.H. Perera, Maharachch. \& K.D. Hyde) Réblová \& A.N. Mill., MycoKeys 74: 28 (2020)

Paragaeumannomyces lapazianus (Carroll \& Munk) Réblová \& A.N. Mill., MycoKeys 74: 31 (2020)

Paragaeumannomyces longisporus (Sacc.) Réblová \& A.N. Mill., MycoKeys 74: 31 (2020)

\section{Paragaeumannomyces} panamensis (Huhndorf \& F.A. Fernández) Réblová \& A.N. Mill., MycoKeys 74: 32 (2020)

Paragaeumannomyces raciborskii (Penz. \& Sacc.) Réblová \& A.N. Mill., MycoKeys 74: 34 (2020)

Paragaeumannomyces rubicundus (Huhndorf \& F.A. Fernández) Réblová \& A.N. Mill., MycoKeys 74: 35 (2020)

Paragalactinia berthetiana (Donadini) Van Vooren, Ascomycete.org 12 (4): 189 (2020)

Paragalactinia eriniae (M.E. Sm.) M.E. Sm. \& Van Vooren, Ascomycete.org 12 (4): 189 (2020)

Paragalactinia infossa (Fogel \& States) Van Vooren, Ascomycete.org 12 (4): 189 (2020)

Paragalactinia infuscata (Quél.) Van Vooren, Ascomycete.org 12 (4): 189 (2020)

Paragalactinia michelii (Boud.) Van Vooren, Ascomycete.org 12 (4): 189 (2020)

Paragalactinia succosa (Berk.) Van Vooren, Ascomycete.org 12 (4): 189 (2020)

Paragalactinia succosella (Le Gal \& Romagn.) Van Vooren, Ascomycete.org 12 (4): 189 (2020)

Paraleptosphaeria polylepidis (Macía, M.E. Palm \& M.P. Martín) Piątek, Flakus \& Rodr. Flakus, Mycol. Progr. 19 (1): 6 (2020)
Paraleptosphaeria rumicis (Quaedvl., Verkley \& Crous) Voglmayr, MycoKeys 66: 16 (2020)

Paraloratospora gahniae (Crous) Thiyagaraja, Bundhun \& K.D. Hyde, Fungal Diversity 100: 105 (2020)

Paraphoma convolvuli (Dearn. \& House) Gomzhina \& Gasich, Mycol. Progr. 19 (3): 192 (2020)

Pararoussoella mangrovei (Phukhams. \& K.D. Hyde) Phukhams. \& K.D. Hyde, Fungal Diversity 10.1007/s13225-020-00448-4: 111 (2020)

Parmelinella afrocetrata (Elix, Eb. Fischer \& Killmann) Marcelli \& A.A. Spielm., Plant and Fungal Systematics 65 (2): 501 (2020)

Parmotrema acanthifolium (Pers.) A.A. Spielm. \& Marcelli, Plant and Fungal Systematics 65 (2): 414 (2020)

Parmotrema concors (Kremp.) A.A. Spielm. \& Marcelli, Plant and Fungal Systematics 65 (2): 426 (2020)

Parmotrema foliolosum (C.W. Dodge) A.A. Spielm. \& Marcelli, Plant and Fungal Systematics 65 (2): 442 (2020)

Parmotrema granulare (Asahina) A.A. Spielm. $\&$ Marcelli, Plant and Fungal Systematics 65 (2): 444 (2020)

Parmotrema lividotessellatum (Hue) A.A. Spielm. \& Marcelli, Plant and Fungal Systematics 65 (2): 451 (2020)

Parmotrema magnum (Lynge) A.A. Spielm. \& Marcelli, Plant and Fungal Systematics 65 (2): 454 (2020)

Parmotrema maximum (Hue) A.A. Spielm. \& Marcelli, Plant and Fungal Systematics 65 (2): 459 (2020)

Parmotrema nudum (Hue) A.A. Spielm. \& Marcelli, Plant and Fungal Systematics 65 (2): 463 (2020)

Parmotrema petropoliense (Zahlbr.) A.A. Spielm. \& Marcelli, Plant and Fungal Systematics 65 (2): 469 (2020) 
Parmotrema radiatum (Lynge) A.A. Spielm. \& Marcelli, Plant and Fungal Systematics 65 (2): 472 (2020)

Parmotrema reterimulosum (J. Steiner \& Zahlbr.) A.A. Spielm. \& Marcelli, Plant and Fungal Systematics 65 (2): 478 (2020)

Parmotrema sieberi (C.W. Dodge) A.A. Spielm. \& Marcelli, Plant and Fungal Systematics 65 (2): 482 (2020)

Parmotrema warmingii (Vain.) A.A. Spielm. \& Marcelli, Plant and Fungal Systematics 65 (2): 496 (2020)

Patellaria australis (Speg.) Baral, Index Fungorum 454: 2 (2020)

Penicillago kabunica (Baghd.) Houbraken, Frisvad \& Samson, Stud. Mycol. 95: 90 (2020)

Penicillago mirabilis (Beliakova \& Milko) Houbraken, Frisvad \& Samson, Stud. Mycol. 95: 90 (2020)

Penicillago moldavica (Milko \& Beliakova) Houbraken, Frisvad \& Samson, Stud. Mycol. 95: 90 (2020)

Penicillium ser. Alutacea (Pitt) Houbraken \& Frisvad, Stud. Mycol. 95: 70 (2020)

Penicillium ser. Crustacea (Pitt) Houbraken \& Frisvad, Stud. Mycol. 95: 80 (2020)

Penicillium ser. Erubescentia (Pitt) Houbraken \& Frisvad, Stud. Mycol. 95: 70 (2020)

Penicillium ser. Lapidosa (Pitt) Houbraken \& Frisvad, Stud. Mycol. 95: 71 (2020)

Penicillium ser. Pinetorum (Pitt) Houbraken \& Frisvad, Stud. Mycol. 95: 57 (2020)

Penicillium ser. Tularensia (Pitt) Houbraken \& Frisvad, Stud. Mycol. 95: 78 (2020)

Pentagenella akompsa (Tuck.) Perlmutter, LaGreca, Ertz \& Tehler, Bryologist 123 (3): 510 (2020)

Petchia mantidicola (Kobayasi \& Shimizu) Thanakitp., Mongkols. \& Luangsa-ard, Persoonia 44: 154 (2020)

Petrakia fagi (C.Z. Wei, Y. Harada \& Katum.)
Beenken, Andr. Gross \& Queloz, Mycol. Progr. 19 (5): 431 (2020)

Petrakia minima (A. Hashim. \& Kaz. Tanaka) Beenken, Andr. Gross \& Queloz, Mycol. Progr. 19 (5): 433 (2020)

Peziza campbellii (Sacc.) Vizzini, Medardi \& Forin, Mycol. Progr. 19 (5): 515 (2020)

*Phacidium anomalum (Nag Raj) W.J. Li \& K.D. Hyde, Fungal Diversity 100: 641 (2020) *Phacidium foliicola (Lib.) W.J. Li \& K.D. Hyde, Fungal Diversity 100: 647 (2020)

*Phacidium subcorticale (Fuckel) W.J. Li \& K.D. Hyde, Fungal Diversity 100: 648 (2020) Phaeohelotium epigaeum (P. Karst.) Baral \& Friebes, Index Fungorum 428: 2 (2020)

Phaeopezia apiculata f. alba (Van Vooren \& Moyne) Van Vooren, Ascomycete.org 12 (4): 189 (2020)

Phaeoplaca camptidia (Tuck.) Søchting, Arup \& Bungartz, Plant and Fungal Systematics 65 (2): 547 (2020)

Phaeoplaca ochrolechioides (S.Y. Kondr. \& Kärnefelt) Søchting \& Arup, Plant and Fungal Systematics 65 (2): 547 (2020)

Phaeopterula anomala (P. Roberts) LealDutra, Dentinger \& G.W. Griff., IMA Fungus 11 (no. 2): 13 (2020)

Phaeopterula stipata (Corner) Leal-Dutra, Dentinger \& G.W. Griff., IMA Fungus 11 (no. 2): 13 (2020)

Phaeopterula taxiformis (Mont.) Leal-Dutra, Dentinger \& G.W. Griff., IMA Fungus 11 (no. 2): 13 (2020)

Phaeopterula taxiformis var. gracilis (Corner) LealDutra, Dentinger \& G.W. Griff., IMA Fungus 11 (no. 2): 13 (2020)

Phaeopterula taxiformis var. taxiformis (Mont.) Leal-Dutra, Dentinger \& G.W. Griff., IMA Fungus 11 (no. 2): 13 (2020)

Phakopsora pipturi (Syd.) Aime \& McTaggart, Fungal Systematics and Evolution 7: 37 (2020)

Phialocephala heterosperma (Le Gal) Tanney 
\& Seifert, Stud. Mycol. 95: 349 (2020)

Phialocephala lignicola (Hern.-Restr., J. Mena \& Gené) Tanney \& Seifert, Stud. Mycol. 95: 351 (2020)

Phialomyces arenicola (Chalab.) Houbraken, Frisvad \& Samson, Stud. Mycol. 95: 90 (2020)

Phialomyces humicoloides (Bills \& Heredia) Houbraken, Frisvad \& Samson, Stud. Mycol. 95: 90 (2020)

Phlebiopsis amethystea (Hjortstam \& Ryvarden) R.S. Chikowski \& C.R.S. Lira, Mycol. Progr. 19: 35 (2020)

*Phormopsora isabellina (Vain.) Elvebakk, S.G. Hong \& C.H. Park, Mycol. Progr. 19 (11): 1359 (2020)

*Phragmidium warburgianum (Henn.) Y.M. Liang \& Y. Liu, Mycologia 112 (4): 749 (2020)

Phragmocapnias plumeriae (Hongsanan \& K.D. Hyde) Abdollahz. \& Crous, Stud. Mycol. 95: 404 (2020)

*Phyllocharis orbicularis (Fr.) S.H. Jiang, Lücking \& Sérus., Fungal Diversity 10.1007/s13225-020-00462-6: 133 (2020)

Phyllocraterina nuda (Zahlbr.) Lücking \& Sérus., Fungal Diversity 10.1007/s13225020-00462-6: 133 (2020)

Phyllocraterina papuana (Sérus. \& Aptroot) Sérus. \& Aptroot, Fungal Diversity 10.1007/s13225-020-00462-6: 133 (2020)

*Phylloporis austropunctata (P.M. McCarthy) S.H. Jiang, Lücking \& J.C. Wei, Fungal Diversity 102: 288 (2020)

*Phylloporis cinefaciens (Nyl.) S.H. Jiang, Lücking \& Sérus., Fungal Diversity 10.1007/s13225-020-00462-6: 134 (2020)

*Phylloporis hypothallina (R.C. Harris) S.H. Jiang, Lücking \& Sérus., Fungal Diversity 10.1007/s13225-020-00462-6: 134 (2020)

*Phylloporis radiata (Lücking) S.H. Jiang, Lücking \& J.C. Wei, Fungal Diversity 102: 289 (2020)
*Phylloporis vulgaris (Müll. Arg.) S.H. Jiang, Lücking \& J.C. Wei, Fungal Diversity 102: 289 (2020)

Phylloscypha boltonii (Quél.) Van Vooren \& Hairaud, Ascomycete.org 12 (4): 189 (2020)

Phylloscypha coquandii (Donadini) Van Vooren, Ascomycete.org 12 (4): 189 (2020)

Phylloscypha labessiana (Boud.) Van Vooren,

T. Richter \& M. Vega, Ascomycete.org 12 (4): 189 (2020)

Phylloscypha phyllogena (Cooke) Van Vooren, Ascomycete.org 12 (4): 189 (2020)

Phylloscypha retrocurvatoides (Van Vooren) Van Vooren, Ascomycete.org 12 (4): 189 (2020)

*Physciella poeltii (Frey) D. Liu \& J.S. Hur, Index Fungorum 464: 1 (2020)

Physconia muscigena var. isidiomuscigena (Essl.) Starosta \& D. Svoboda, Lichenologist 52 (4): 313 (2020)

*Pinaceicola cordae (Koukol) Crous, M. Shen \& Y. Zhang ter, Stud. Mycol. 96: 208 (2020)

*Pinaceicola pini (Crous \& de Hoog) Crous, M. Shen \& Y. Zhang ter, Stud. Mycol. 96: 208 (2020)

Piptoporellus baudonii (Pat.) Tibuhwa, Ryvarden \& S. Tibell, Mycologia 112 (5): 1021 (2020)

Pisutiella congrediens (Nyl.) S.Y. Kondr., Lőkös \& Farkas, Acta bot. hung. 62 (1-2): 122 (2020)

Pisutiella conversa (Kremp.) S.Y. Kondr., Lökös \& Farkas, Acta bot. hung. 62 (1-2): 122 (2020)

Pisutiella furax (Egea \& Llimona) S.Y. Kondr., Lökös \& Farkas, Acta bot. hung. 62 (1-2): 122 (2020)

Pisutiella grimmiae (Nyl.) S.Y. Kondr., Lőkös \& Farkas, Acta bot. hung. 62 (1-2): 122 (2020)

Pisutiella ivanpisutii (S.Y. Kondr., Lőkös \& Hur) S.Y. Kondr., Lőkös \& Farkas, Acta bot. hung. 62 (1-2): 122 (2020) 
Pisutiella phaeothamnos (Kalb \& Poelt) S.Y. Kondr., Lőkös \& Farkas, Acta bot. hung. 62 (1-2): 122 (2020)

Plenodomus visci (Moesz) Gruyter, Aveskamp \& Verkley, Fungal Systematics and Evolution 6: 213 (2020)

Plicaria lundellii (Donadini) Van Vooren, Cahiers de la FMBDS 7: 24 (2020)

Podila clonocystis (W. Gams) Vandepol \& Bonito, Fungal Diversity 10.1007/s13225020-00455-5: 18 (2020)

Podila epicladia (W. Gams \& Emden) Vandepol \& Bonito, Fungal Diversity 10.1007/s13225-020-00455-5: 18 (2020)

Podila epigama (W. Gams \& Domsch) Vandepol \& Bonito, Fungal Diversity 10.1007/s13225-020-00455-5: 18 (2020)

Podila horticola (Linnem.) Vandepol \& Bonito, Fungal Diversity 10.1007/s13225-02000455-5: 18 (2020)

Podila humilis (Linnem. ex W. Gams) Vandepol \& Bonito, Fungal Diversity 10.1007/s13225-020-00455-5: 18 (2020)

Podila minutissima (Tiegh.) Vandepol \& Bonito, Fungal Diversity 10.1007/s13225020-00455-5: 18 (2020)

Podila verticillata (Linnem.) Vandepol \& Bonito, Fungal Diversity 10.1007/s13225020-00455-5: 19 (2020)

Podoscypha curta (Fr) Ryvarden, Syn. Fung. 40: 75 (2020)

Podospora costaricensis (G.C. Carroll \& Munk) Y. Marín, A.N. Mill. \& Stchigel, Microorganisms 8 (9, no. 1430): 21 (2020)

Podospora sacchari (B.M. Robison) Y. Marín $\&$ Stchigel, Microorganisms 8 (9, no. 1430): 21 (2020)

Podospora striatispora (Furuya \& Udagawa) Y. Marín \& Stchigel, Microorganisms 8 (9, no. 1430): 21 (2020)

Polyozosia actophila (Wedd.) S.Y. Kondr., Lőkös \& Farkas, Acta bot. hung. 62 (1-2): $100(2020)$
Polyozosia agardhiana (Ach.) S.Y. Kondr., Lőkös \& Farkas, Acta bot. hung. 62 (1-2): 100 (2020)

Polyozosia altunica (R. Mamut \& A. Abbas) S.Y. Kondr., Lőkös \& Farkas, Acta bot. hung. 62 (1-2): 101 (2020)

Polyozosia antiqua (J.R. Laundon) S.Y. Kondr., Lőkös \& Farkas, Acta bot. hung. 62 (1-2): 101 (2020)

Polyozosia bandolensis (B. de Lesd.) S.Y. Kondr., Lőkös \& Farkas, Acta bot. hung. 62 (1-2): 101 (2020)

Polyozosia behringii (Nyl.) S.Y. Kondr., Lőkös \& Farkas, Acta bot. hung. 62 (1-2): 101 (2020)

Polyozosia caesioalutacea (H. Magn.) S.Y. Kondr., Lökös \& Farkas, Acta bot. hung. 62 (1-2): 101 (2020)

Polyozosia carlottiana (Lewis \& Śliwa) S.Y. Kondr., Lőkös \& Farkas, Acta bot. hung. 62 (1-2): 101 (2020)

Polyozosia congesta (Clauzade \& Vězda) S.Y. Kondr., Lőkös \& Farkas, Acta bot. hung. 62 (1-2): 101 (2020)

Polyozosia eurycarpa (Poelt, Leuckert \& Cl. Roux) S.Y. Kondr., Lökös \& Farkas, Acta bot. hung. 62 (1-2): 101 (2020)

Polyozosia expectans (Darb.) S.Y. Kondr., Lőkös \& Farkas, Acta bot. hung. 62 (1-2): 101 (2020)

Polyozosia flowersiana (H. Magn.) S.Y. Kondr., Lőkös \& Farkas, Acta bot. hung. 62 (1-2): 102 (2020)

Polyozosia fugiens (Nyl.) S.Y. Kondr., Lőkös \& Farkas, Acta bot. hung. 62 (1-2): 102 (2020)

Polyozosia invadens (H. Magn.) S.Y. Kondr., Lőkös \& Farkas, Acta bot. hung. 62 (1-2): 102 (2020)

Polyozosia juniperina (Śliwa) S.Y. Kondr., Lőkös \& Farkas, Acta bot. hung. 62 (1-2): 102 (2020)

Polyozosia latzelii (Zahlbr.) S.Y. Kondr., 
Lőkös \& Farkas, Acta bot. hung. 62 (1-2): $102(2020)$

Polyozosia liguriensis (B. de Lesd.) S.Y. Kondr., Lökös \& Farkas, Acta bot. hung. 62 (1-2): 102 (2020)

Polyozosia massei (M. Bertrand \& J.-Y. Monnat) S.Y. Kondr., Lőkös \& Farkas, Acta bot. hung. 62 (1-2): 102 (2020)

Polyozosia mons-nivis (Darb.) S.Y. Kondr., Lőkös \& Farkas, Acta bot. hung. 62 (1-2): 102 (2020)

Polyozosia oyensis (M.-P. Bertrand \& $\mathrm{Cl}$. Roux) S.Y. Kondr., Lőkös \& Farkas, Acta bot. hung. 62 (1-2): 102 (2020)

Polyozosia percrenata (H. Magn.) S.Y. Kondr., Lökös \& Farkas, Acta bot. hung. 62 (1-2): 102 (2020)

Polyozosia persimilis (Th. Fr.) S.Y. Kondr., Lőkös \& Farkas, Acta bot. hung. 62 (1-2): 103 (2020)

Polyozosia poeltiana (Clauzade \& Cl. Roux) S.Y. Kondr., Lőkös \& Farkas, Acta bot. hung. 62 (1-2): 103 (2020)

Polyozosia prominens (Clauzade \& Vězda) S.Y. Kondr., Lőkös \& Farkas, Acta bot. hung. 62 (1-2): 103 (2020)

Polyozosia prophetae-eliae (Sipman) S.Y. Kondr., Lőkös \& Farkas, Acta bot. hung. 62 (1-2): 103 (2020)

Polyozosia salina (H. Magn.) S.Y. Kondr., Lökös \& Farkas, Acta bot. hung. 62 (1-2): 103 (2020)

Polyozosia schofieldii (Brodo) S.Y. Kondr., Lőkös \& Farkas, Acta bot. hung. 62 (1-2): 103 (2020)

Polyozosia sverdrupiana (Øvstedal) S.Y. Kondr., Lőkös \& Farkas, Acta bot. hung. 62 (1-2): 103 (2020)

Polyozosia torrida (Vain.) S.Y. Kondr., Lökös \& Farkas, Acta bot. hung. 62 (1-2): 103 (2020)

Polyozosia wetmorei (Śliwa) S.Y. Kondr., Lőkös \& Farkas, Acta bot. hung. 62 (1-2):
104 (2020)

Polyozosia zosterae (Ach.) S.Y. Kondr., Lőkös \& Farkas, Acta bot. hung. 62 (1-2): 104 (2020)

Polyporus gracilisporus (H. Lee, N.K. Kim \& Y.W. Lim) Bernicchia \& Gorjón, Romar: 616 (2020)

Porodaedalea abietis (P. Karst.) Bernicchia \& Gorjón, Romar: 571 (2020)

Porostereum submembranaceum (Henn) Ryvarden, Syn. Fung. 40: 92 (2020)

Porphyrellus olivaceobrunneus (Zeller \& F.D. Bailey) Klofac, Öst. Z. Pilzk. 28: 19 (2020)

*Protocandelariella blastidiata (Yakovchenko) D. Liu, Hur \& S.Y. Kondr., Acta bot. hung. 62 (3-4): 305 (2020)

*Protocandelariella subdeflexa (Nyl.) Poelt, D. Liu, Hur \& S.Y. Kondr., Acta bot. hung. 62 (3-4): 305 (2020)

Pruniphilomyces circumscissus (Sacc.) Crous \& Bulgakov, Fungal Systematics and Evolution 6: 215 (2020)

Prunulus diosmus (Krieglst. \& Schwöbel) C. Hahn, Die Pilze Deutschlands: 188 (2020)

Psathyrella sachaensis (Singer) Voto, Boll. Assoc. Micol. Ecol. Romana 109 (1): 12 (2020)

Psathyrella sect. Lutenses (Kits van Wav.) Voto, Rivista Micologica Romana, Boll. AMER 110 (2): 89 (2020)

*Pseudobactrodesmium longisporum (M.B. Ellis) W. Dong \& K.D. Hyde, Frontiers in Microbiology 11 (no. 565): 8 (2020)

Pseudobogoriella alata (Groenh. ex Aptroot) Lücking, R. Miranda \& Aptroot, Fungal Diversity $\quad 10.1007 / \mathrm{s} 13225-020-00462-6$ : 172 (2020)

Pseudobogoriella annonacea (Müll. Arg.) Lücking, R. Miranda \& Aptroot, Fungal Diversity $\quad$ 10.1007/s13225-020-00462-6: 172 (2020)

Pseudobogoriella captiosa (Kremp.) Lücking, R. Miranda \& Aptroot, Fungal Diversity 
10.1007/s13225-020-00462-6: 172 (2020)

Pseudobogoriella exigua (Müll. Arg.) Lücking, R. Miranda \& Aptroot, Fungal Diversity 10.1007/s13225-020-00462-6: 172 (2020)

Pseudobogoriella fumosula (Zahlbr.) Lücking, R. Miranda \& Aptroot, Fungal Diversity 10.1007/s13225-020-00462-6: 172 (2020)

Pseudobogoriella hemisphaerica (Müll. Arg.) Lücking, R. Miranda \& Aptroot, Fungal Diversity $\quad 10.1007 / \mathrm{s} 13225-020-00462-6$ : 172 (2020)

Pseudobogoriella lateralis (Sipman) Lücking, R. Miranda \& Aptroot, Fungal Diversity 10.1007/s13225-020-00462-6: 172 (2020)

Pseudobogoriella leuckertii (D. Hawksw. \& J.C. David) Lücking, R. Miranda \& Aptroot, Fungal Diversity 10.1007/s13225-02000462-6: 172 (2020)

Pseudobogoriella minutula (Zahlbr.) Lücking, R. Miranda \& Aptroot, Fungal Diversity 10.1007/s13225-020-00462-6: 172 (2020)

Pseudobogoriella nonensis (Stirt.) Lücking, $R$. Miranda \& Aptroot, Fungal Diversity 10.1007/s13225-020-00462-6: 172 (2020)

Pseudobogoriella pachnea (Körb.) Lücking, R. Miranda \& Aptroot, Fungal Diversity 10.1007/s13225-020-00462-6: 172 (2020)

Pseudobogoriella punctata (Aptroot) Lücking, R. Miranda \& Aptroot, Fungal Diversity 10.1007/s13225-020-00462-6: 173 (2020)

Pseudobogoriella socialis (Zahlbr.) Lücking, R. Miranda \& Aptroot, Fungal Diversity 10.1007/s13225-020-00462-6: 173 (2020)

Pseudobogoriella striguloides (Sérus. \& Aptroot) Lücking, R. Miranda \& Aptroot, Fungal Diversity 10.1007/s13225-02000462-6: 173 (2020)

Pseudobogoriella subfallens (Müll. Arg.) Lücking, R. Miranda \& Aptroot, Fungal Diversity $\quad 10.1007 / \mathrm{s} 13225-020-00462-6$ : 173 (2020)

Pseudoboletus parasiticus var. piperatoides (J. Blum) C. Hahn, Die Pilze Deutschlands: 324
(2020)

*Pseudocoleophoma flavescens (Gruyter, Noordel. \& Boerema) W.J. Li \& K.D. Hyde, Fungal Diversity 100: 676 (2020)

Pseudoechria curvicolla (G. Winter) Y. Marín, A.N. Mill. \& Stchigel, Microorganisms 8 (9, no. 1430): 30 (2020)

Pseudoechria decidua (Cailleux) Y. Marín, A.N. Mill. \& Stchigel, Microorganisms 8 (9, no. 1430): 30 (2020)

Pseudoechria longicollis (L.M. Ames) Y. Marín \& Stchigel, Microorganisms 8 (9, no. 1430): 30 (2020)

Pseudoechria prolifica (Cailleux) Y. Marín, A.N. Mill. \& Stchigel, Microorganisms 8 (9, no. 1430): 30 (2020)

Pseudohamigera striata (Raper \& Fennell) Houbraken, Frisvad \& Samson, Stud. Mycol. 95: 90 (2020)

*Pseudojahnula potamophila (K.D. Hyde \& S.W. Wong) W. Dong, H. Zhang \& K.D. Hyde, Fungal Diversity 10.1007/s13225020-00463-5: 35 (2020)

Pseudolaccaria fellea (Peck) Vizzini, Matheny, Consiglio

\& M. Marchetti, Fungal Diversity 101: 238 (2020)

Pseudomarasmius glabrocystidiatus (Antonin, Ryoo, Ka) R.H. Petersen, Mycotaxon 135: 37 (2020)

Pseudomarasmius nidus-avis (Cesar, Bandala, Montoya) R.H. Petersen, Mycotaxon 135: 38 (2020)

Pseudomarasmius pallidocephalus (Gilliam) R.H. Petersen, Mycotaxon 135: 45 (2020)

Pseudomarasmius straminipes (Peck) R.H. Petersen, Mycotaxon 135: 83 (2020)

Pseudoneoconiothyrium euonymi (Crous \& Akulov) Phukhams. \& K.D. Hyde, Fungal Diversity 10.1007/s13225-020-00448-4: 112 (2020)

Pseudoomphalina intermedia (Kauffman) 
Voitk, I. Saar, Lebeuf \& Kennedy, Botany 98 (2): 99 (2020)

*Pseudopeyronellaea eucalypti (Crous \& M.J. Wingf.) L.W. Hou, L. Cai \& Crous, Stud. Mycol. 96: 339 (2020)

Pseudoroussoella elaeicola (Konta \& K.D. Hyde) Mapook \& K.D. Hyde, Fungal Diversity 101: 89 (2020)

Pseudoschizothecium atropurpureum (A.N. Mill. \& Huhndorf) Y. Marín, A.N. Mill. \& Stchigel, Microorganisms 8 (9, no. 1430): 32 (2020)

*Pseudosigmoidea excentrica (R.F. Castañeda, W. Gams \& Saikawa) Crous, M. Shen \& Y. Zhang ter, Stud. Mycol. 96: 210 (2020)

Pseudosperma alboflavellum (C.K. Pradeep \& Matheny) Haelew., Index Fungorum 436: 1 (2020)

Pseudosperma friabile (Matheny \& Kudzma) Haelew., Index Fungorum 436: 1 (2020)

Pseudosperma neglectum (E. Horak, Matheny \& Desjardin) Haelew., MycoKeys 69: 25 (2020)

Pseudosperma obsoletum (Quadr.) Valade, Index Fungorum 447: 1 (2020)

Pseudothyridariella mahakoshae (Devadatha, V.V. Sarma, D.N. Wanas., K.D. Hyde \& E.B.G. Jones) Mapook \& K.D. Hyde, Fungal Diversity 101: 100 (2020)

Psilachnum miniatum (Kanouse) Baral, Index Fungorum 454: 2 (2020)

Psilachnum rubicundum (Sacc. \& Speg.) Baral, Monogr. Orbiliomycetes: 1684 (2020)

Psilachnum schoenoplecti (Raitv. \& P. Blank) Baral, Index Fungorum 454: 2 (2020)

Pterulicium argentinum (Speg.) Leal-Dutra, Dentinger \& G.W. Griff., IMA Fungus 11 (no. 2): 14 (2020)

Pterulicium argentinum var. argentinum (Speg.) Leal-Dutra, Dentinger \& G.W. Griff., IMA Fungus 11 (no. 2): 14 (2020)

Pterulicium argentinum var. ramosum (Corner) Leal-Dutra, Dentinger \& G.W.
Griff., Index Fungorum 435: 1 (2020)

Pterulicium argentinum var. ramosum (Corner) Leal-Dutra, Dentinger \& G.W. Griff., IMA Fungus 11 (no. 2): 14 (2020)

Pterulicium bambusae (Corner) Leal-Dutra, Dentinger \& G.W. Griff., IMA Fungus 11 (no. 2): 14 (2020)

Pterulicium bromeliphilum (Corner) LealDutra, Dentinger \& G.W. Griff., IMA Fungus 11 (no. 2): 14 (2020)

Pterulicium brunneosetosum (Corner) LealDutra, Dentinger \& G.W. Griff., IMA Fungus 11 (no. 2): 14 (2020)

Pterulicium campoi (Speg.) Leal-Dutra, Dentinger \& G.W. Griff., IMA Fungus 11 (no. 2): 14 (2020)

Pterulicium caricis-pendulae (Corner) LealDutra, Dentinger \& G.W. Griff., IMA Fungus 11 (no. 2): 14 (2020)

Pterulicium crassisporum (P. Roberts) LealDutra, Dentinger \& G.W. Griff., IMA Fungus 11 (no. 2): 14 (2020)

Pterulicium cystidiatum (Corner) Leal-Dutra, Dentinger \& G.W. Griff., IMA Fungus 11 (no. 2): 14 (2020)

Pterulicium debile (Corner) Leal-Dutra, Dentinger \& G.W. Griff., Index Fungorum 435: 1 (2020)

Pterulicium debile (Corner) Leal-Dutra, Dentinger \& G.W. Griff., IMA Fungus 11 (no. 2): 14 (2020)

Pterulicium echo (D.J. McLaughlin \& E.G. McLaughlin) Leal-Dutra, Dentinger \& G.W. Griff., IMA Fungus 11 (no. 2): 15 (2020)

Pterulicium epiphylloides (Corner) Leal-Dutra, Dentinger \& G.W. Griff., IMA Fungus 11 (no. 2): 15 (2020)

Pterulicium epiphyllum (Corner) Leal-Dutra, Dentinger \& G.W. Griff., IMA Fungus 11 (no. 2): 15 (2020)

Pterulicium fasciculare (Bres. \& Pat.) LealDutra, Dentinger \& G.W. Griff., IMA Fungus 11 (no. 2): 15 (2020) 
Pterulicium fluminense (Corner) Leal-Dutra, Dentinger \& G.W. Griff., IMA Fungus 11 (no. 2): 15 (2020)

Pterulicium gordium (Speg.) Leal-Dutra, Dentinger \& G.W. Griff., IMA Fungus 11 (no. 2): 15 (2020)

Pterulicium gordium var. gordium (Speg.) Leal-Dutra, Dentinger \& G.W. Griff., IMA Fungus 11 (no. 2): 15 (2020)

Pterulicium gordium var. macrosporum (Corner) Leal-Dutra, Dentinger \& G.W. Griff., IMA Fungus 11 (no. 2): 15 (2020)

Pterulicium gracile (Desm. \& Berk.) LealDutra, Dentinger \& G.W. Griff., IMA Fungus 11 (no. 2): 15 (2020)

Pterulicium incarnatum (Pat.) Leal-Dutra, Dentinger \& G.W. Griff., IMA Fungus 11 (no. 2): 16 (2020)

Pterulicium intermedium (Dogma) Leal-Dutra, Dentinger \& G.W. Griff., IMA Fungus 11 (no. 2): 16 (2020)

Pterulicium laxum (Pat.) Leal-Dutra, Dentinger \& G.W. Griff., IMA Fungus 11 (no. 2): 16 (2020)

Pterulicium lilaceobrunneum (Corner) LealDutra, Dentinger \& G.W. Griff., IMA Fungus 11 (no. 2): 16 (2020)

Pterulicium lilaceobrunneum var. evolutius (Corner) Leal-Dutra, Dentinger \& G.W. Griff., IMA Fungus 11 (no. 2): 16 (2020)

Pterulicium lilaceobrunneum var. lilaceobrunneum (Corner) Leal-Dutra, Dentinger \& G.W. Griff., IMA Fungus 11 (no. 2): 16 (2020)

Pterulicium longisporum (Corner) Leal-Dutra, Dentinger \& G.W. Griff., IMA Fungus 11 (no. 2): 16 (2020)

Pterulicium macrosporum (Pat.) Leal-Dutra, Dentinger \& G.W. Griff., IMA Fungus 11 (no. 2): 16 (2020)

Pterulicium majus (Corner) Leal-Dutra, Dentinger \& G.W. Griff., IMA Fungus 11 (no. 2): 17 (2020)
Pterulicium mangiforme (Corner) Leal-Dutra, Dentinger \& G.W. Griff., IMA Fungus 11 (no. 2): 17 (2020)

Pterulicium microsporum (Corner) LealDutra, Dentinger \& G.W. Griff., IMA Fungus 11 (no. 2): 17 (2020)

Pterulicium nanum (Pat.) Leal-Dutra, Dentinger \& G.W. Griff., IMA Fungus 11 (no. 2): 17 (2020)

Pterulicium naviculum (Corner) Leal-Dutra, Dentinger \& G.W. Griff., IMA Fungus 11 (no. 2): 17 (2020)

Pterulicium oryzae (Remsberg) Leal-Dutra, Dentinger \& G.W. Griff., IMA Fungus 11 (no. 2): 17 (2020)

Pterulicium phyllodicola (Corner) Leal-Dutra, Dentinger \& G.W. Griff., IMA Fungus 11 (no. 2): 17 (2020)

Pterulicium phyllophilum (McAlpine) LealDutra, Dentinger \& G.W. Griff., IMA Fungus 11 (no. 2): 17 (2020)

Pterulicium rigidum (Donk) Leal-Dutra, Dentinger \& G.W. Griff., IMA Fungus 11 (no. 2): 17 (2020)

Pterulicium sclerotiicola (Berthier) LealDutra, Dentinger \& G.W. Griff., IMA Fungus 11 (no. 2): 18 (2020)

Pterulicium secundirameum (Lév) Leal-Dutra, Dentinger \& G.W. Griff., IMA Fungus 11 (no. 2): 18 (2020)

Pterulicium sprucei (Mont.) Leal-Dutra, Dentinger \& G.W. Griff., IMA Fungus 11 (no. 2): 18 (2020)

Pterulicium subsimplex (Henn.) Leal-Dutra, Dentinger \& G.W. Griff., IMA Fungus 11 (no. 2): 18 (2020)

Pterulicium subsimplex var. multifidum (Corner) Leal-Dutra, Dentinger \& G.W. Griff., IMA Fungus 11 (no. 2): 18 (2020)

Pterulicium subsimplex var. subsimplex (Henn.) Leal-Dutra, Dentinger \& G.W. Griff., IMA Fungus 11 (no. 2): 18 (2020)

Pterulicium subtyphuloides (Corner) Leal- 
Dutra, Dentinger \& G.W. Griff., IMA Fungus 11 (no. 2): 18 (2020)

Pterulicium sulcisporum (Corner) Leal-Dutra, Dentinger \& G.W. Griff., IMA Fungus 11 (no. 2): 18 (2020)

Pterulicium tenuissimum (M.A. Curtis) LealDutra, Dentinger \& G.W. Griff., IMA Fungus 11 (no. 2): 19 (2020)

Pterulicium typhuloides (Corner) Leal-Dutra, Dentinger \& G.W. Griff., IMA Fungus 11 (no. 2): 19 (2020)

Pterulicium typhuloides var. minor (Corner) Leal-Dutra, Dentinger \& G.W. Griff., IMA Fungus 11 (no. 2): 19 (2020)

Pterulicium typhuloides var. typhuloides (Corner) Leal-Dutra, Dentinger \& G.W. Griff., IMA Fungus 11 (no. 2): 19 (2020)

Pterulicium ulmi (Peck) Leal-Dutra, Dentinger \& G.W. Griff., IMA Fungus 11 (no. 2): 19 (2020)

Pterulicium velutipes (Corner) Leal-Dutra, Dentinger \& G.W. Griff., IMA Fungus 11 (no. 2): 19 (2020)

*Puccinia caulophylli (Kom.) Jing X. Ji \& Kakishima, Mycotaxon 134 (4): 726 (2020)

*Puiggariella confluens (Müll. Arg.) S.H. Jiang, Lücking \& J.C. Wei, Fungal Diversity 102: 281 (2020)

*Puiggariella hypothelia (Nyl.) S.H. Jiang, Lücking \& Sérus., Fungal Diversity 10.1007/s13225-020-00462-6: 135 (2020)

*Puiggariella nemathora (Mont.) S.H. Jiang, Lücking \& J.C. Wei, Fungal Diversity 102: 282 (2020)

*Puiggariella nigrocincta (Müll. Arg.) S.H. Jiang, Lücking \& J.C. Wei, Fungal Diversity 102: 284 (2020)

Pulverulina ulmicola (H.E. Bigelow) Matheny \& K.W. Hughes, Southeastern Naturalist 19 (3): 452 (2020)

Punjabia pakistanica (Usman \& Khalid) D. Wächt. \& A. Melzer, Mycol. Progr. 19 (11): 1235 (2020)
Purpureodiscus (G. Hirsch) Van Vooren, Ascomycete.org 12 (4): 189 (2020)

Purpureodiscus bananincola (Rehm) Van Vooren, Ascomycete.org 12 (4): 190 (2020) Purpureodiscus kreiselii (G. Hirsch) Van Vooren, Ascomycete.org 12 (4): 190 (2020)

Purpureodiscus luteorosellus (Le Gal) Van Vooren, Ascomycete.org 12 (4): 190 (2020)

Purpureodiscus subisabellinus (Le Gal) Van Vooren, Ascomycete.org 12 (4): 190 (2020)

Purpureodiscus subisabellinus f. ianthinus (Grelet ex Le Gal) Van Vooren, Ascomycete.org 12 (4): 190 (2020)

Purpureodiscus subisabellinus f. subisabellinus (Le Gal) Van Vooren, Ascomycete.org 12 (4): 190 (2020)

Purpureomyces khaoyaiensis (Hywel-Jones) Luangsa-ard, Samson \& Thanakitp., Stud. Mycol. 95: 241 (2020)

*Pycnopeziza americana (Nag Raj) W.J. Li \& K.D. Hyde, Fungal Diversity 100: 705 (2020)

Pyrenodesmia aetnensis (B. de Lesd.) S.Y. Kondr., Acta bot. hung. 62 (1-2): 122 (2020) Pyrenodesmia albolutescens (Nyl.) S.Y. Kondr., Acta bot. hung. 62 (1-2): 122 (2020) Pyrenodesmia albopruinosa (Arnold) S.Y. Kondr., Acta bot. hung. 62 (3-4): 280 (2020) Pyrenodesmia aractina (Fr.) S.Y. Kondr., Acta bot. hung. 62 (1-2): 122 (2020)

Pyrenodesmia atroflava (Turner) S.Y. Kondr., Acta bot. hung. 62 (1-2): 122 (2020)

Pyrenodesmia bicolor (H. Magn.) S.Y. Kondr., Acta bot. hung. 62 (1-2): 123 (2020)

Pyrenodesmia ceracea (J.R. Laundon) S.Y. Kondr., Acta bot. hung. 62 (3-4): 280 (2020) Pyrenodesmia cretensis (Zahlbr.) S.Y. Kondr., Acta bot. hung. 62 (3-4): 280 (2020)

Pyrenodesmia erythrocarpa (Pers.) S.Y. Kondr., Acta bot. hung. 62 (3-4): 280 (2020) Pyrenodesmia haematites (Chaub. ex St.Amans) S.Y. Kondr., Acta bot. hung. 62 (34): 280 (2020)

Pyrenodesmia molariformis (Frolov, Vondrák, 
Nadyeina \& Khodos.) S.Y. Kondr., Acta bot. hung. 62 (1-2): 123 (2020)

Pyrenodesmia neotaurica (Vondrák, Khodos., Arup \& Søchting) S.Y. Kondr., Acta bot. hung. 62 (1-2): 123 (2020)

Pyrenodesmia peliophylla (Tuck.) S.Y. Kondr., Acta bot. hung. 62 (1-2): 123 (2020)

Pyrenodesmia percrocata (Arnold) S.Y. Kondr., Acta bot. hung. 62 (3-4): 280 (2020)

Pyrenodesmia soralifera (Vondrák \& Hrouzek)

S.Y. Kondr., Acta bot. hung. 62 (3-4): 280 (2020)

Pyrenodesmia transcaspica (Nyl.) S.Y. Kondr., Acta bot. hung. 62 (3-4): 280 (2020)

Pyrenodesmia viridirufa (Ach.) S.Y. Kondr., Acta bot. hung. 62 (3-4): 280 (2020)

Pyrenodesmia xerica (Poelt \& Vězda) S.Y. Kondr., Acta bot. hung. 62 (3-4): 280 (2020) Pyrenopeziza aquosa (Berk. \& Broome) Baral, Index Fungorum 428: 2 (2020)

*Pyrrhulomyces astragalinus (Fr.) E.J. Tian \& Matheny, Mycologia 10.1080/00275514.2020.1816067: 11 (2020)

Rachicladosporium iridis (Auersw.) Crous, Fungal Systematics and Evolution 6: 216 (2020)

*Racoplaca maculata (Cooke \& Massee) S.H. Jiang, Lücking \& J.C. Wei, Fungal Diversity 102: 286 (2020)

*Racoplaca melanobapha (Kremp.) S.H. Jiang, Lücking \& J.C. Wei, Fungal Diversity 10.1007/s13225-020-00462-6: 136 (2020)

*Racoplaca melanobapha (Kremp.) S.H. Jiang, Lücking \& J.C. Wei, Fungal Diversity 102: 286 (2020)

*Racoplaca transversoundulata (Sipman) S.H. Jiang, Lücking \& J.C. Wei, Fungal Diversity 102: 287 (2020)

*Racoplaca tremens (Müll. Arg.) S.H. Jiang, Lücking \& J.C. Wei, Fungal Diversity 102: 287 (2020)

Rajchenbergia mangrovei (Y.C. Dai, X.H. Ji \& J. Vlasák) Salvador-Montoya, Drechsler-
Santos \& Popoff, Pl. Syst. Evol. 306 (no. 34): 24 (2020)

Rajchenbergia pertenuis (Xavier de Lima \& Oliveira-Filho) Salvador-Montoya, Popoff \& Drechsler-Santos, Pl. Syst. Evol. 306 (no. 34): 24 (2020)

Rajchenbergia tenuissima (H.Y. Yu, C.L. Zhao \& Y.C. Dai) Salvador-Montoya, Drechsler-Santos \& Popoff, Pl. Syst. Evol. 306 (no. 34): 24 (2020)

Ramaria fennica f. olivacea (Schild) Franchi \& M. Marchetti, Index Fungorum 437: 1 (2020)

Ramaria flavoides var. abetonensis (Franchi \& M. Marchetti) Franchi \& M. Marchetti, Index Fungorum 437: 1 (2020)

Ramaria formosa f. neoformosa (R.H. Petersen) Franchi \& M. Marchetti, Index Fungorum 437: 1 (2020)

Ramaria intimorosea f. flavipes (Franchi \& M. Marchetti) Franchi \& M. Marchetti, Index Fungorum 437: 1 (2020)

Ramaria luteoaurantiaca f. citrina (Schild) Franchi \& M. Marchetti, Index Fungorum 437: 1 (2020)

Ramaria praecox var. luteovernalis (Franchi, M. Marchetti \& Bottoni) Franchi, M. Marchetti \& Bottoni, Index Fungorum 437: 1 (2020)

Ramaria stricta f. violaceotincta (Bourdot \& Galzin) Franchi \& M. Marchetti, Index Fungorum 437: 1 (2020)

Ramaria violacea (Schild) Franchi \& M. Marchetti, Index Fungorum 437: 1 (2020)

Ramularia digitalis (Fuckel) U. Braun, Schlechtendalia 37: 12 (2020)

Rasamsonia oblata (Pitt \& A.D. Hocking) Yanai \& Udagawa, Japanese Journal of Mycology 61 (2): 93 (2020)

Rasamsonia sabulosa (Pitt \& A.D. Hocking) Yanai \& Udagawa, Japanese Journal of Mycology 61 (2): 96 (2020)

Rehmanniella leucoxantha (Müll. Arg.) S.Y. 
Kondr., Acta bot. hung. 62 (3-4): 280 (2020)

Rehmanniella subgyalectoides (S.Y. Kondr. \&

I. Kärnefelt) S.Y. Kondr., Acta bot. hung. 62 (3-4): 280 (2020)

Rehmanniella syvashica (Khodos., Vondrák \& Soun) S.Y. Kondr., Acta bot. hung. 62 (3-4): 280 (2020)

Reichlingia anombrophila (Coppins \& $\mathrm{P}$. James) Frisch, Graphis Scripta 32 (1): 15 (2020)

Reichlingia dendritica (Leight.) Ertz \& Sanderson, Lichenologist 52: 265 (2020)

*Remotididymella capsici (Bond.-Mont.) L.W. Hou, L. Cai \& Crous, Stud. Mycol. 96: 337 (2020)

Resinoscypha monoseptata (R. Galán \& Raitv.) T. Kosonen, Huhtinen \& K. Hansen, Persoonia 46: 58 (2020)

Resinoscypha variepilosa (R. Galán \& Raitv.) T. Kosonen, Huhtinen \& K. Hansen, Persoonia 46: 58 (2020)

\section{Rhexocercosporidium microsporum}

(Ekanayaka \& K.D. Hyde) Phutthacharoen \& K.D. Hyde, Fungal Diversity 100: 153 (2020)

*Rhizoplaca callichroa (Zahlbr.) Y.Y. Zhang, MycoKeys 66: 144 (2020)

*Rhizoplaca pachyphylla (H. Magn.) Y.Y. Zhang, MycoKeys 66: 148 (2020)

*Rhodofomitopsis monomitica (Yuan Y. Chen) B.K. Cui, Yuan Y. Chen \& Shun Liu, Fungal Diversity 10.1007/s13225-020-00461-7: 140 (2020)

*Rhodofomitopsis oleracea (R.W. Davidson \& Lombard) B.K. Cui, Yuan Y. Chen \& Shun Liu, Fungal Diversity 10.1007/s13225-02000461-7: 140 (2020)

Rhynchobrunnera lolii (K.M. King, J.S. West, P.C. Brunner, P.S. Dyer \& Fitt) B.A. McDonald, U. Braun \& Crous, Fungal Systematics and Evolution 7: 85 (2020)

Rhynchobrunnera orthospora (Caldwell) B.A. McDonald, U. Braun \& Crous, Fungal
Systematics and Evolution 7: 85 (2020)

*Rhynchogastrema complexa (Landell, Pagnocca, Sette, Passarini, K.M. Garcia, J.R.A. Ribeiro, C.F. Lee, L.R. Brandão, C.A. Rosa \& P. Valente) Xin Zhan Liu, F.Y. Bai, M. Groenew., Boekhout \& Yurkov, Stud. Mycol. 96: 136 (2020)

*Rhynchogastrema fermentans (C.F. Lee) Xin Zhan Liu, F.Y. Bai, M. Groenew., Boekhout \& Yurkov, Stud. Mycol. 96: 136 (2020)

*Rhynchogastrema glucofermentans (S.O. Suh \& M. Blackw.) Xin Zhan Liu, F.Y. Bai, M. Groenew., Boekhout \& Yurkov, Stud. Mycol. 96: 136 (2020)

*Rhynchogastrema tunnelae (Boekhout, Fell, Scorzetti \& Theelen) Xin Zhan Liu, F.Y. Bai, M. Groenew., Boekhout \& Yurkov, Stud. Mycol. 96: 136 (2020)

*Rhynchogastrema visegradensis (G. Péter \& Dlauchy) Xin Zhan Liu, F.Y. Bai, M. Groenew., Boekhout \& Yurkov, Stud. Mycol. 96: 136 (2020)

Rhypophila cochleariformis (Cailleux) Y. Marín, A.N. Mill. \& Guarro, Microorganisms 8 (9, no. 1430): 17 (2020)

Rhypophila decipiens (G. Winter) Y. Marín, A.N. Mill. \& Guarro, Microorganisms 8 (9, no. 1430): 17 (2020)

Rhypophila myriospora (P. Crouan \& $\mathrm{H}$. Crouan) Y. Marín, A.N. Mill. \& Guarro, Microorganisms 8 (9, no. 1430): 17 (2020)

Rhypophila pleiospora (G. Winter) Y. Marín, A.N. Mill. \& Guarro, Microorganisms 8 (9, no. 1430): 17 (2020)

Rodwayella haematoidea (Cooke \& W. Phillips) Baral \& Quijada, Monogr. Orbiliomycetes: 1673 (2020)

Rogerpetersonia torreyae (Bonar) Aime \& McTaggart, Fungal Systematics and Evolution 7: 30 (2020)

Roridomyces pruinosoviscidus (Corner) Blanco-Dios, Index Fungorum 449: 1 (2020) 
Roridomyces pruinosoviscidus var. pruinosoviscidus (Corner) Blanco-Dios, Index Fungorum 449: 1 (2020)

Roridomyces pruinosoviscidus var. rabaulensis (Corner) Blanco-Dios, Index Fungorum 449: 1 (2020)

Roridomyces sublucens (Corner) Blanco-Dios, Index Fungorum 449: 1 (2020)

Rossmanomyces monesis (Ziller) Aime \& McTaggart, Fungal Systematics and Evolution 7: 32 (2020)

Rossmanomyces pyrolae (Rostr.) Aime \& McTaggart, Fungal Systematics and Evolution 7: 34 (2020)

Rossmanomyces ramischiae (Lagerh.) Aime \& McTaggart, Fungal Systematics and Evolution 7: 34 (2020)

Rugiboletus andinus (Halling) Halling \& B. Ortiz, Mycologia 112 (1): 207 (2020)

Rutstroemia pseudosydowiana (Yan J. Zhao \& Hosoya) Quijada \& Baral, Index Fungorum 454: 1 (2020)

Saitozyma ninhbinhensis (D.T. Luong, M. Takash., Dung \& Nakase) Yurkov, Stud. Mycol. 96: 137 (2020)

*Salmonomyces acalyphae (F.L. Tai) L. Kiss, D.N. Jin \& S.Y. Liu, Frontiers in Microbiology 11 (no. 1571): 14 (2020)

*Samsoniella hepiali (Q.T. Chen \& R.Q. Dai ex R.Q. Dai, X.M. Li, A.J. Shao, Shu F. Lin, J.L. Lan, Wei H. Chen \& C.Y. Shen) H. Yu, R.Q. Dai, Y.B. Wang, Y. Wang \& Zhu L. Yang, Fungal Diversity 103: 31 (2020)

*Sanguinoderma bataanense (Murrill) Y.F. Sun \& B.K. Cui, Persoonia 44: 224 (2020)

*Sanguinoderma elmerianum (Murrill) Y.F. Sun \& B.K. Cui, Persoonia 44: 225 (2020)

*Sanguinoderma perplexum (Corner) Y.F. Sun \& B.K. Cui, Persoonia 44: 230 (2020)

*Sanguinoderma rude (Berk.) Y.F. Sun, D.H. Costa \& B.K. Cui, Persoonia 44: 233 (2020)

*Sanguinoderma rugosum (Blume \& T. Nees) Y.F. Sun, D.H. Costa \& B.K. Cui, Persoonia
44: 235 (2020)

*Sarcogyne canadensis (H. Magn.) K. Knudsen, J.N. Adams, Kocourk. \& Y. Wang, Bryologist 123 (1): 21 (2020)

Sarcoleotia cinnamomea (Maas Geest.) Baral, Index Fungorum 428: 2 (2020)

Sarcopodium radians (Penz. \& Sacc.) Forin \& Vizzini, Persoonia 45: 244 (2020)

Sarcopodium tjibodense (Penz. \& Sacc.) Forin \& Vizzini, Persoonia 45: 246 (2020)

Saxiloba firmula (Müll. Arg.) Lücking, Moncada \& Sipman, Plant and Fungal Systematics 65 (2): 580 (2020)

Schizothecium selenosporum (Stchigel, Guarro \& M. Calduch) Y. Marín \& Stchigel, Microorganisms 8 (9, no. 1430): 34 (2020)

Schummia angulata (Aptroot \& Schumm)

Lücking, R. Miranda \& Aptroot, Fungal Diversity $\quad 10.1007 / \mathrm{s} 13225-020-00462-6$ : 174 (2020)

Sclerenchymomyces jonesii (Wanas., Camporesi \& K.D. Hyde) Phukhams. \& K.D. Hyde, Fungal Diversity 10.1007/s13225020-00448-4: 43 (2020)

*Sclerotiophoma versabilis (Boerema, Loer. \& Hamers) L.W. Hou, L. Cai \& Crous, Stud. Mycol. 96: 351 (2020)

Scolecobasidium ailanthi (Jayasiri, E.B.G. Jones \& K.D. Hyde) Crous, Fungal Systematics and Evolution 7: 15 (2020)

*Scolecobasidium aquaticum (Samerp., Gloyna, Gerrits van den Ende \& de Hoog) Crous, M. Shen \& Y. Zhang ter, Stud. Mycol. 96: 210 (2020)

*Scolecobasidium atlanticum (A.M. Wellman) Crous, M. Shen \& Y. Zhang ter, Stud. Mycol. 96: 210 (2020)

*Scolecobasidium bacilliforme (Samerp., Gerrits van den Ende, Menken \& de Hoog) Crous, M. Shen \& Y. Zhang ter, Stud. Mycol. 96: 210 (2020)

*Scolecobasidium capsici (Crous \& R. Cheew.) Crous, M. Shen \& Y. Zhang ter, Stud. Mycol. 
96: 210 (2020)

*Scolecobasidium cordanae (Samerp., Crous \& de Hoog) Crous, M. Shen \& Y. Zhang ter, Stud. Mycol. 96: 210 (2020)

*Scolecobasidium dracaenae (Crous) Crous, M. Shen \& Y. Zhang ter, Stud. Mycol. 96: 211 (2020)

*Scolecobasidium globale (Samerp., Duarte, Attili-Angelis \& de Hoog) Crous, M. Shen \& Y. Zhang ter, Stud. Mycol. 96: 211 (2020)

*Scolecobasidium icarus (Samerp., Giraldo, Guarro \& de Hoog) Crous, M. Shen \& Y. Zhang ter, Stud. Mycol. 96: 211 (2020)

*Scolecobasidium macrozamiae (Crous \& R.G. Shivas) Crous, M. Shen \& Y. Zhang ter, Stud. Mycol. 96: 211 (2020)

*Scolecobasidium minimum (Fassat.) Crous,

M. Shen \& Y. Zhang ter, Stud. Mycol. 96: 212 (2020)

*Scolecobasidium musicola (Crous) Crous, M.

Shen \& Y. Zhang ter, Stud. Mycol. 96: 212 (2020)

*Scolecobasidium olivaceum (A. Giraldo, Gené, Deanna A. Sutton \& Guarro) Crous, M. Shen \& Y. Zhang ter, Stud. Mycol. 96: 212 (2020)

*Scolecobasidium pandanicola (Crous \& M.J. Wingf.) Crous, M. Shen \& Y. Zhang ter, Stud. Mycol. 96: 212 (2020)

*Scolecobasidium phaeophorum (Samerp., Gerrits van den Ende, Menken \& de Hoog) Crous, M. Shen \& Y. Zhang ter, Stud. Mycol. 96: 212 (2020)

*Scolecobasidium podocarpi (Crous) Crous, M. Shen \& Y. Zhang ter, Stud. Mycol. 96: 212 (2020)

*Scolecobasidium ramosum (A. Giraldo, Gené, Deanna A. Sutton \& Guarro) Crous, M. Shen \& Y. Zhang ter, Stud. Mycol. 96: 212 (2020)

*Scolecobasidium robustum (Samerp., Gerrits van den Ende, Menken \& de Hoog) Crous, M. Shen \& Y. Zhang ter, Stud. Mycol. 96:
212 (2020)

* Scolecobasidium sexuale (Samerp., Van der Linde \& de Hoog) Crous, M. Shen \& Y. Zhang ter, Stud. Mycol. 96: 212 (2020)

*Scolecobasidium verrucosum (Zachariah, Sankaran \& Leelav.) Crous, M. Shen \& Y. Zhang ter, Stud. Mycol. 96: 212 (2020)

Scutellinia legaliae var. peniculospora (Donadini) Van Vooren, Cahiers de la FMBDS 7: 48 (2020)

Seifertia alpina (Höhn.) Beenken, Andr. Gross \& Queloz, Mycol. Progr. 19 (5): 436 (2020) *Sidera minutipora (Rodway \& Cleland) Y.C. Dai, F. Wu, G.M. Gates \& Rui Du, MycoKeys 68: 120 (2020)

Siphulopsis queenslandica (Kantvilas) Kantvilas \& A.R. Nilsen, Lichenologist 52 (3): 218 (2020)

*Skeletocutis albomarginata (Zipp. ex Lév.) Rui Du \& Y.C. Dai, Mycosystema 39 (4): 643 (2020)

*Skeletocutis indica (Ganesh \& Ryvarden) Rui Du \& Y.C. Dai, Mycosystema 39 (4): 644 (2020)

*Skeletocutis sajanensis (Parmasto) Rui Du \& Y.C. Dai, Mycosystema 39 (4): 644 (2020)

*Spathaspora jeffriesii (N.H. Nguyen, S.O. Suh \& M. Blackw.) C.Y. Chai \& F.L. Hui, MycoKeys 75: 44 (2020)

*Spathaspora materiae (A.C. Barbosa, R.M. Cadete, F.C.O. Gomes, Lachance \& Rosa) C.Y. Chai \& F.L. Hui, MycoKeys 75: 44 (2020)

Squamulea flakusii (Wilk) Arup, Søchting \& Bungartz, Plant and Fungal Systematics 65 (2): 564 (2020)

Squamulea loekoesiana (S.Y. Kondr. \& D. Upreti) Arup, Søchting \& Bungartz, Plant and Fungal Systematics 65 (2): 569 (2020)

Squamulea phyllidizans (Wetmore) Søchting $\&$ Bungartz, Plant and Fungal Systematics 65 (2): 567 (2020)

*Stagonospora cylindrica (B. Sutton \& Alcorn) 
W.J. Li \& K.D. Hyde, Fungal Diversity 100: $739(2020)$

*Stagonosporopsis nemophilae (Neerg.) L.W. Hou, L. Cai \& Crous, Stud. Mycol. 96: 324 (2020)

Steccherinum aurantilaetum (Corner) Bernicchia \& Gorjón, Romar: 795 (2020)

Steccherinum imbricatum (Spirin) Bernicchia \& Gorjón, Romar: 801 (2020)

Steccherinum microporum (Spirin, Zmitr. \& Malysheva) Bernicchia \& Gorjón, Romar: 803 (2020)

Steineropsis laceratula (Hue) T. Sprib. \& Ekman, Lichenologist 52 (2): 134 (2020)

Stemphylium rosae (Wanas., Camporesi, E.B.G. Jones \& K.D. Hyde) Phukhams. \& K.D. Hyde, Fungal Diversity 10.1007/s13225-020-00448-4: 93 (2020)

Stemphylium rosae-caninae (Wanas., Camporesi, E.B.G. Jones \& K.D. Hyde) Phukhams. \& K.D. Hyde, Fungal Diversity 10.1007/s13225-020-00448-4: 93 (2020)

Sticta flavireagens (Gyeln.) Diederich \& Ertz, Plant and Fungal Systematics 65 (1): 65 (2020)

*Strigula cylindrospora (Syd. \& P. Syd.) W.J. Li \& K.D. Hyde, Fungal Diversity 100: 748 (2020)

*Stropharia scabella (Zeller) E.J. Tian \& M. Gordon, Mycologia 10.1080/00275514.2020.1816067: 14 (2020)

Sucioplaca diplacia (Ach.) Bungartz, Søchting \& Arup, Plant and Fungal Systematics 65 (2): 548 (2020)

Suillus albivelatus f. pseudoalbivelatus (B. Ortiz \& Lodge) Klofac, Öst. Z. Pilzk. 28: 21 (2020)

Suillus americanus f. himalayensis (B. Verma \& M.S. Reddy) Klofac, Öst. Z. Pilzk. 28: 19 (2020)

Suillus elbensis var. serotinus (Frost) Klofac, Öst. Z. Pilzk. 28: 21 (2020)

Suillus flavidus f. megaporinus (Snell \& E.A.
Dick) Klofac, Öst. Z. Pilzk. 28: 21 (2020)

Sungia yongmunensis (G.H. Sung, J.M. Sung \& Spatafora) Luangsa-ard, Thanakitp. \& Samson, Stud. Mycol. 95: 243 (2020)

*Swinscowia affinis (A. Massal.) S.H. Jiang, Lücking \& Sérus., Fungal Diversity 10.1007/s13225-020-00462-6: 137 (2020)

*Swinscowia albicascens (Nyl.) S.H. Jiang, Lücking \& Sérus., Fungal Diversity 10.1007/s13225-020-00462-6: 137 (2020)

*Swinscowia albolinita (Nyl.) S.H. Jiang, Lücking \& Sérus., Fungal Diversity 10.1007/s13225-020-00462-6: 137 (2020)

*Swinscowia alpestris (Vězda) S.H. Jiang, Lücking \& Sérus., Fungal Diversity 10.1007/s13225-020-00462-6: 137 (2020)

*Swinscowia amphora (Aptroot \& Lücking) S.H. Jiang, Lücking \& Sérus., Fungal Diversity 10.1007/s13225-020-00462-6: 138 (2020)

*Swinscowia aquatica (H. Harada) S.H. Jiang, Lücking \& Sérus., Fungal Diversity 10.1007/s13225-020-00462-6: 138 (2020)

*Swinscowia australiensis (P.M. McCarthy) S.H. Jiang, Lücking \& Sérus., Fungal Diversity 10.1007/s13225-020-00462-6: 138 (2020)

*Swinscowia bahamensis (Riddle) S.H. Jiang, Lücking \& Sérus., Fungal Diversity 10.1007/s13225-020-00462-6: 138 (2020)

*Swinscowia bispora (Aptroot \& K.H. Moon) S.H. Jiang, Lücking \& Sérus., Fungal Diversity $\quad 10.1007 / \mathrm{s} 13225-020-00462-6$ : 138 (2020)

*Swinscowia calcarea (Bricaud \& Cl. Roux) S.H. Jiang, Lücking \& Sérus., Fungal Diversity $\quad$ 10.1007/s13225-020-00462-6: 138 (2020)

*Swinscowia cavicola (Cl. Roux \& Bricaud) S.H. Jiang, Lücking \& Sérus., Fungal Diversity 10.1007/s13225-020-00462-6: 138 (2020)

*Swinscowia confusa (Fryday, Coppins \& 
Common) S.H. Jiang, Lücking \& Sérus., Fungal Diversity 10.1007/s13225-02000462-6: 138 (2020)

*Swinscowia decipiens (Malme) S.H. Jiang, Lücking \& Sérus., Fungal Diversity 10.1007/s13225-020-00462-6: 138 (2020)

*Swinscowia divisa (P.M. McCarthy) S.H. Jiang, Lücking \& Sérus., Fungal Diversity 10.1007/s13225-020-00462-6: 138 (2020)

*Swinscowia endolithea (Cl. Roux \& Bricaud) S.H. Jiang, Lücking \& Sérus., Fungal Diversity 10.1007/s13225-020-00462-6: 138 (2020)

*Swinscowia fracticonidia (R.C. Harris) S.H. Jiang, Lücking \& Sérus., Fungal Diversity 10.1007/s13225-020-00462-6: 138 (2020)

*Swinscowia glabra (A. Massal.) S.H. Jiang, Lücking \& Sérus., Fungal Diversity 10.1007/s13225-020-00462-6: 138 (2020)

*Swinscowia griseonitens (R.C. Harris) S.H. Jiang, Lücking \& Sérus., Fungal Diversity 10.1007/s13225-020-00462-6: 139 (2020)

*Swinscowia jamesii (Swinscow) S.H. Jiang, Lücking \& Sérus., Fungal Diversity 10.1007/s13225-020-00462-6: 139 (2020)

*Swinscowia johnsonii (P.M. McCarthy) S.H. Jiang, Lücking \& Sérus., Fungal Diversity 10.1007/s13225-020-00462-6: 139 (2020)

*Swinscowia laceribracae (R.C. Harris) S.H. Jiang, Lücking \& Sérus., Fungal Diversity 10.1007/s13225-020-00462-6: 139 (2020)

*Swinscowia muriconidiata (Aptroot, L.I. Ferraro \& M. Cáceres) S.H. Jiang, Lücking $\&$ Sérus., Fungal Diversity 10.1007/s13225020-00462-6: 139 (2020)

*Swinscowia muriformis (Aptroot \& Diederich) S.H. Jiang, Lücking \& Sérus., Fungal Diversity 10.1007/s13225-02000462-6: 139 (2020)

*Swinscowia muscicola (F. Berger, Coppins, Cl. Roux \& Sérus.) S.H. Jiang, Lücking \& Sérus., Fungal Diversity 10.1007/s13225020-00462-6: 139 (2020)
*Swinscowia obtecta (Vain.) S.H. Jiang, Lücking \& Sérus., Fungal Diversity 10.1007/s13225-020-00462-6: 140 (2020)

*Swinscowia pallida (Aptroot \& K.H. Moon) S.H. Jiang, Lücking \& Sérus., Fungal Diversity 10.1007/s13225-020-00462-6: 140 (2020)

*Swinscowia porinoides (Canals, Boqueras \& Gómez-Bolea) S.H. Jiang, Lücking \& Sérus., Fungal Diversity 10.1007/s13225-02000462-6: 140 (2020)

*Swinscowia rhodinula (Zahlbr.) S.H. Jiang, Lücking \& Sérus., Fungal Diversity 10.1007/s13225-020-00462-6: 140 (2020)

*Swinscowia rostrata (R.C. Harris \& Aptroot) S.H. Jiang, Lücking \& Sérus., Fungal Diversity 10.1007/s13225-020-00462-6: 140 (2020)

*Swinscowia rupestris (P.M. McCarthy) S.H. Jiang, Lücking \& Sérus., Fungal Diversity 10.1007/s13225-020-00462-6: 140 (2020)

*Swinscowia stigmatella (Ach.) S.H. Jiang, Lücking \& Sérus., Fungal Diversity 10.1007/s13225-020-00462-6: 140 (2020)

*Swinscowia submuriformis (R.C. Harris) S.H. Jiang, Lücking \& Sérus., Fungal Diversity 10.1007/s13225-020-00462-6: 140 (2020)

*Swinscowia tagananae (Harm.) S.H. Jiang, Lücking \& Sérus., Fungal Diversity 10.1007/s13225-020-00462-6: 140 (2020)

*Swinscowia thelopsidoides (Coppins, $\mathrm{Cl}$. Roux \& Sérus. ) S.H. Jiang, Lücking \& Sérus., Fungal Diversity 10.1007/s13225020-00462-6: 141 (2020)

*Sympoventuria africana (Crous) Crous, M. Shen \& Y. Zhang ter, Stud. Mycol. 96: 214 (2020)

Synnematotriadelphia stilboidea (Mercado \& R.F. Castañeda) Chuaseehar., Somrith., Nuankaew \& Boonyuen, Mycol. Progr. 19 (2): 133 (2020)

Synnematotriadelphia synnematofera (Matsush.) Chuaseehar., Somrith., 
Nuankaew \& Boonyuen, Mycol. Progr. 19 (2): 133 (2020)

Talaromyces resedanus (McLennan \& Ducker) A.J. Chen, Houbraken \& Samson, MycoKeys 68: 96 (2020)

*Talaromyces resinae (Z.T. Qi \& H.Z. Kong) Houbraken \& X.C. Wang, Stud. Mycol. 95: 91 (2020)

Telimena acalyphae (Bonar) Mardones \& M. Piepenbr., Mycol. Progr. 19 (12): 1589 (2020)

Telimena balansae (Speg.) Mardones, Trampe \& M. Piepenbr., Mycol. Progr. 19 (12): 1593 (2020)

Telimena pichinchae (Petr.) Mardones, Trampe \& M. Piepenbr., Mycol. Progr. 19 (12): 1592 (2020)

Termitomyces songolarum (Courtec.) Furneaux, Index Fungorum 444: 1 (2020)

*Teunia cuniculi (K.S. Shin \& Y.H. Park) Q.M. Wang, F.Y. Bai \& A.H. Li, Stud. Mycol. 96: 86 (2020)

Thekopsora americana (Farl.) Aime \& McTaggart, Fungal Systematics and Evolution 7: 34 (2020)

Thekopsora potentillae (Kom.) Aime \& McTaggart, Fungal Systematics and Evolution 7: 34 (2020)

Thelonectria applanata (Fuckel) U. Braun \& Bensch, Schlechtendalia 37: 88 (2020)

Thermoascus verrucosus (Samson \& Tansey) Houbraken, Frisvad \& Samson, Stud. Mycol. 95: 91 (2020)

Thysanorea asiatica (Hong Y. Su, Udayanga \& K.D. Hyde) Hern.-Restr. \& Crous, Fungal Systematics and Evolution 6: 18 (2020)

Thysanorea curvata (Hong Y. Su, Udayanga \& K.D. Hyde) Hern.-Restr. \& Crous, Fungal Systematics and Evolution 6: 18 (2020)

Thysanorea lotorum (Morgan-Jones) Hern.Restr. \& Crous, Fungal Systematics and Evolution 6: 18 (2020)

Thysanorea melanica (Hong Y. Su, Udayanga
\& K.D. Hyde) Hern.-Restr. \& Crous, Fungal Systematics and Evolution 6: 18 (2020)

Thysanorea nonramosa (X.D. Yu, G.N. Wang \& H. Zhang) Hern.-Restr. \& Crous, Fungal Systematics and Evolution 6: 18 (2020)

Thysanorea obscura (Matsush.) Hern.-Restr. $\&$ Crous, Fungal Systematics and Evolution 6: 18 (2020)

Thysanorea rousseliana (Mont.) Hern.-Restr. $\&$ Crous, Fungal Systematics and Evolution 6: 18 (2020)

Thysanorea thailandensis (W. Dong, H. Zhang \& K.D. Hyde) Hern.-Restr. \& Crous, Fungal Systematics and Evolution 6: 21 (2020)

Toensbergia geminipara (Th. Fr.) T. Sprib. \& Resl, Lichenologist 52 (2): 127 (2020)

Toniniopsis fusispora (Hepp ex Körb.) $\mathrm{Cl}$. Roux, Catalogue des lichens et champignons lichénicoles de France métropolitaine. 3e édition revue et augmentée 1: 1290 (2020)

Trechispora brasiliensis (Corner) K.H. Larss., Mycol. Progr. 19 (12): 1409 (2020)

Trechispora mellina (Bres.) K.H. Larss., Mycol. Progr. 19 (12): 1409 (2020)

Triangularia arizonensis (Griffiths) Y. Marín, A.N. Mill. \& Stchigel, Microorganisms 8 (9, no. 1430): 23 (2020)

Triangularia striata (Ellis \& Everh.) Y. Marín, A.N. Mill. \& Stchigel, Microorganisms 8 (9, no. 1430): 23 (2020)

Triangularia tetraspora (J.N. Rai, Mukerji \& J.P. Tewari) Y. Marín \& Stchigel, Microorganisms 8 (9, no. 1430): 24 (2020)

Trichopezizella japonica (Syd. \& P. Syd.) Tochihara \& Hosoya, Phytotaxa 434 (3): 207 (2020)

Tricladium varicosporioides (Tubaki) P.R. Johnst. \& Baschien, Fungal Systematics and Evolution 6: 240 (2020)

Tropicoporus melleoporus (Murrill) SalvadorMontoya \& Drechsler-Santos, Pl. Syst. Evol. 306 (no. 34): 21 (2020) 
Tuber melanosporum var. hiemalbum (Chatin) Della Maggiora, Micologia Toscana 2: 68 (2020)

Tulosesus allovelus (Uljé) D. Wächt. \& A. Melzer, Mycol. Progr. 19 (11): 1208 (2020)

Tulosesus amphithallus (M. Lange \& A.H. Sm.) D. Wächt. \& A. Melzer, Mycol. Progr. 19 (11): 1208 (2020)

Tulosesus angulatus (Peck) D. Wächt. \& A. Melzer, Mycol. Progr. 19 (11): 1208 (2020)

Tulosesus aokii (Hongo) D. Wächt. \& A. Melzer, Mycol. Progr. 19 (11): 1208 (2020)

Tulosesus bisporiger (Buller ex P.D. Orton) D. Wächt. \& A. Melzer, Mycol. Progr. 19 (11): 1208 (2020)

Tulosesus bisporus (J.E. Lange) D. Wächt. \& A. Melzer, Mycol. Progr. 19 (11): 1208 (2020)

Tulosesus brevisetulosus (Arnolds) D. Wächt. \& A. Melzer, Mycol. Progr. 19 (11): 1209 (2020)

Tulosesus callinus (M. Lange \& A.H. Sm.) D. Wächt. \& A. Melzer, Mycol. Progr. 19 (11): 1209 (2020)

Tulosesus canistri (Uljé \& Verbeken) D. Wächt. \& A. Melzer, Mycol. Progr. 19 (11): 1209 (2020)

Tulosesus christianopolitanus (Örstadius \& E. Larss.) D. Wächt. \& A. Melzer, Mycol. Progr. 19 (11): 1210 (2020)

Tulosesus cinereopallidus (L. Nagy, Házi, Papp \& Vágvölgyi) D. Wächt. \& A. Melzer, Mycol. Progr. 19 (11): 1210 (2020)

Tulosesus cinnamomeotinctus (P.D. Orton) D. Wächt. \& A. Melzer, Mycol. Progr. 19 (11): 1210 (2020)

Tulosesus congregatus (Bull.) D. Wächt. \& A. Melzer, Mycol. Progr. 19 (11): 1211 (2020)

Tulosesus doverii (L. Nagy) D. Wächt. \& A. Melzer, Mycol. Progr. 19 (11): 1211 (2020)

Tulosesus ephemerus (Bull.) D. Wächt. \& A. Melzer, Mycol. Progr. 19 (11): 1211 (2020) Tulosesus eurysporus (M. Lange \& A.H. Sm.)
D. Wächt. \& A. Melzer, Mycol. Progr. 19 (11): 1211 (2020)

Tulosesus fallax (M. Lange \& A.H. Sm.) D. Wächt. \& A. Melzer, Mycol. Progr. 19 (11): $1211(2020)$

Tulosesus fuscocystidiatus (L. Nagy, Házi, Papp \& Vágvölgyi) D. Wächt. \& A. Melzer, Mycol. Progr. 19 (11): 1211 (2020)

Tulosesus heterosetulosus (Locq. ex Watling) D. Wächt. \& A. Melzer, Mycol. Progr. 19 (11): 1211 (2020)

Tulosesus heterothrix (Kühner) D. Wächt. \& A. Melzer, Mycol. Progr. 19 (11): 1211 (2020)

Tulosesus hiascens (Fr.) D. Wächt. \& A. Melzer, Mycol. Progr. 19 (11): 1212 (2020)

Tulosesus impatiens (Fr.) D. Wächt. \& A. Melzer, Mycol. Progr. 19 (11): 1212 (2020)

Tulosesus marculentus (Britzelm.) D. Wächt. \& A. Melzer, Mycol. Progr. 19 (11): 1212 (2020)

Tulosesus minutisporus (Uljé) D. Wächt. \& A. Melzer, Mycol. Progr. 19 (11): 1212 (2020)

Tulosesus mitrinodulisporus (Doveri \& Sarrocco) D. Wächt. \& A. Melzer, Mycol. Progr. 19 (11): 1212 (2020)

Tulosesus pallidus (L. Nagy, Házi, Papp \& Vágvölgyi) D. Wächt. \& A. Melzer, Mycol. Progr. 19 (11): 1212 (2020)

Tulosesus pellucidus (P. Karst.) D. Wächt. \& A. Melzer, Mycol. Progr. 19 (11): 1212 (2020)

Tulosesus plagioporus (Romagn.) D. Wächt. \& A. Melzer, Mycol. Progr. 19 (11): 1212 (2020)

Tulosesus pseudoamphithallus (Uljé) D. Wächt. \& A. Melzer, Mycol. Progr. 19 (11): 1212 (2020)

Tulosesus radicellus (J. Házi, L. Nagy, T. Papp \& C. Vágvölgyi) D. Wächt. \& A. Melzer, Mycol. Progr. 19 (11): 1212 (2020)

Tulosesus sabulicola (L. Nagy, Házi, Papp \& Vágvölgyi) D. Wächt. \& A. Melzer, Mycol. 
Progr. 19 (11): 1212 (2020)

Tulosesus sassii (M. Lange \& A.H. Sm.) D. Wächt. \& A. Melzer, Mycol. Progr. 19 (11): $1212(2020)$

Tulosesus sclerocystidiosus (M. Lange \& A.H. Sm.) D. Wächt. \& A. Melzer, Mycol. Progr. 19 (11): 1212 (2020)

Tulosesus singularis (Uljé) D. Wächt. \& A. Melzer, Mycol. Progr. 19 (11): 1212 (2020)

Tulosesus subdisseminatus (M. Lange) D. Wächt. \& A. Melzer, Mycol. Progr. 19 (11): $1212(2020)$

Tulosesus subimpatiens (M. Lange \& A.H. Sm.) D. Wächt. \& A. Melzer, Mycol. Progr. 19 (11): 1212 (2020)

Tulosesus subpurpureus (A.H. Sm.) D. Wächt. \& A. Melzer, Mycol. Progr. 19 (11): 1213 (2020)

Tulosesus uljei (L. Nagy, Házi, T. Papp \& Vágvölgyi) D. Wächt. \& A. Melzer, Mycol. Progr. 19 (11): 1213 (2020)

Tulosesus velatopruinatus (Bender) D. Wächt. \& A. Melzer, Mycol. Progr. 19 (11): 1213 (2020)

Typhula podocarpi (Crous) Olariaga, Huhtinen, Læssøe, J.H. Petersen \& K. Hansen, Stud. Mycol. 96: 177 (2020)

*Tyrannosorus hanlinianus (U. Braun \& Feiler) Crous, M. Shen \& Y. Zhang ter, Stud. Mycol. 96: 224 (2020)

*Tyrannosorus hystrioides (Dugan, R.G. Roberts \& Hanlin) Crous, M. Shen \& Y. Zhang ter, Stud. Mycol. 96: 224 (2020)

Unguiculella incarnatina (Quél.) Baral, Index Fungorum 428: 2 (2020)

Upretia hueana (B. de Lesd.) S.Y. Kondr. \& Upreti, Acta bot. hung. 62 (3-4): 380 (2020)

Uromyces chenopodii-fruticosi (DC.) M. Abbasi \& Aime, Mycologia 112 (3): 548 (2020)

*Vacuiphoma ferulae (Pat.) L.W. Hou, L. Cai \& Crous, Stud. Mycol. 96: 371 (2020)

*Vacuiphoma laurina (Thüm.) L.W. Hou, L.
Cai \& Crous, Stud. Mycol. 96: 371 (2020)

*Varioseptispora hodgkissii (W.H. Ho, Yanna \& K.D. Hyde) Z.H. Xu, Jian Ma, X.G. Zhang \& R.F. Castañeda, Mycotaxon 135 (4): 757 (2020)

*Varioseptispora versiseptatis (M.K.M. Wong, Goh \& K.D. Hyde) Z.H. Xu, Jian Ma, X.G. Zhang \& R.F. Castañeda, Mycotaxon 135 (4): 757 (2020)

*Venturia peltigericola (Crous \& Diederich) Crous, M. Shen \& Y. Zhang ter, Stud. Mycol. 96: 274 (2020)

Vermilacinia granulans (Sipman) R. Spjut \& Sérus., MycoKeys 74: 110 (2020)

*Verruconis terricola (J. Ren, C.Y. Jie, Y.L. Jiang, K.D. Hyde \& Yong Wang bis) Crous, M. Shen \& Y. Zhang ter, Stud. Mycol. 96: 215 (2020)

Verruculopsis beltraminiana (A. Massal.) $\mathrm{Cl}$. Roux, Catalogue des lichens et champignons lichénicoles de France métropolitaine. 3e édition revue et augmentée 1: 1290 (2020)

Vesiculozygosporium echinosporum (Bunting \& E.W. Mason) Crous, Fungal Systematics and Evolution 6: 227 (2020)

Vexillomyces atrovirens (Pers.) Baral, Quijada \& G. Marson, Index Fungorum 454: 1 (2020)

Vexillomyces canariensis (Ouell. \& Korf) Quijada \& Baral, Index Fungorum 454: 1 (2020)

Vexillomyces clavatus (Ouell. \& Korf) Quijada \& Baral, Index Fungorum 454: 1 (2020)

Vexillomyces dacrymycetoideus (Ouell. \& Korf) Quijada \& Baral, Index Fungorum 454: 1 (2020)

Vexillomyces hydnicola (Berk. \& Broome) Baral \& Marson, Index Fungorum 454: 1 (2020)

Vexillomyces imperspicuus (Sacc., E. Bommer \& M. Rousseau) Quijada \& Baral, Index Fungorum 454: 1 (2020)

Vexillomyces pleomorphicus (Gamundí \& Giaiotti) Quijada \& Baral, Index Fungorum 
454: 1 (2020)

Vexillomyces pseudotsugae (J.W. Groves) Baral, Index Fungorum 454: 1 (2020)

Vexillomyces xylophilus (Kirschst.) Baral, G. Marson \& Quijada, Index Fungorum 454: 1 (2020)

Wetmoreana brouardii (B. de Lesd.) Wilk \& Søchting, Plant and Fungal Systematics 65 (2): 562 (2020)

*Xenasmatella ailaoshanensis (C.L. Zhao) C.L. Zhao \& T.K. Zong, Phytotaxa 489 (2): 118 (2020)

Xenasmatella alnicola (Bourdot \& Galzin) K.H. Larss. \& Ryvarden, Syn. Fung. 40: 116 (2020)

Xenonectriella angulospora (Etayo) F. Berger, Herzogia 33: 483 (2020)

Xerocomellus amylosporus (A.H. Sm.) J.L. Frank \& N. Siegel, Fungal Systematics and Evolution 6: 267 (2020)

Xerocomellus mendocinensis (Theirs) $\mathrm{N}$. Siegel, C.F. Schwarz \& J.L. Frank, Fungal Systematics and Evolution 6: 282 (2020)

Xerocomus leptospermi (McNabb) Klofac, Öst. Z. Pilzk. 28: 21 (2020)
Xerophorus (Bon) Vizzini, Consiglio \& M. Marchetti, Fungal Diversity 101: 239 (2020)

Xerophorus donadinii (Bon) Vizzini, Consiglio \& M. Marchetti, Fungal Diversity 101: 241 (2020)

Xerophorus olivascens (Boud.) Vizzini, Consiglio \& M. Marchetti, Fungal Diversity 101: 239 (2020)

Xylobolus peculiare (Parmasto, Boidin \& Dhingra) Ryvarden, Syn. Fung. 40: 86 (2020)

Yosiokobayasia kusanaginensis (Kobayasi \& Shimizu) Samson, Luangsa-ard \& Thanakitp., Stud. Mycol. 95: 243 (2020)

Zaghouania notelaeae (Syd.) Aime \& McTaggart, Fungal Systematics and Evolution 7: 30 (2020)

Zasmidium persicae (T. Yokoy. \& Nasu) Thapboualy, Souvannasane, Phengs. \& Karun., Stud. Fung. 5 (1): 354 (2020)

Zygosaccharomyces osmophilus (Kreger-van Rij) T.T.S.Matos, J.F. Teixeira, L.G. Matias, A.R.O. Santos, S.O. Suh, Barrio, Lachance \& C.A. Rosa, Int. J. Syst. Evol. Microbiol. 70 (5): 3377 (2020)

\section{修订名称 Replacement names}

Acarospora lendemeri K. Knudsen \& Kocourk., Opuscula Philolichenum 19: 159 (2020)

Alnicola indica Blanco-Dios, Index Fungorum 449: 1 (2020)

Amanita albogrisescens f. separata Contu ex Hanss, Bull. Soc. mycol. Fr. 133: 106 (2020) Amphisphaeria neoaquatica Samarak., Maharachch. \& K.D. Hyde, Journal of Fungi 6 (3): 18 (2020)

Aspergillus chaetosartoryae Hubka, Kocsubé \& Houbraken, Stud. Mycol. 95: 86 (2020)

Clitopilus papuanus Blanco-Dios, Yesca 32: 75 (2020)

Collybia ochracea Para, Micol. Veg. Medit. 34:
135 (2020)

Dirinaria rhodocladonica Kalb, Schumm \& Elix, Australas. Lichenol. 86: 8 (2020)

Entoloma danbullense Blanco-Dios, Index Fungorum 449: 1 (2020)

Entoloma heae Blanco-Dios, Index Fungorum 449: 1 (2020)

Entoloma karstedtiae Blanco-Dios, Index Fungorum 458: 1 (2020)

Entoloma tennesseense Blanco-Dios, Yesca 32: 75 (2020)

Erysiphe flexibilis M. Bradshaw, U. Braun \& S. Takam., Fungal Systematics and Evolution 7: 55 (2020)

Fomitiporia junipericola Rivoire \& Pirlot, 
Polypores de France et d'Europe: 372 (2020) Fusarium cucurbiticola O'Donnell, Geiser, Kasson \& T. Aoki, Index Fungorum 440: 2 (2020)

Fusarium helgardnirenbergiae O'Donnell, Geiser, Kasson \& T. Aoki, Index Fungorum 440: 2 (2020)

Fusarium ngaiotongaense O'Donnell, Geiser, Kasson \& T. Aoki, Index Fungorum 440: 3 (2020)

Fusarium quercinum O'Donnell, Geiser, Kasson \& T. Aoki, Index Fungorum 440: 4 (2020)

Fusarium solani-melongenae O'Donnell, Geiser, Kasson \& T. Aoki, Index Fungorum 440: 4 (2020)

Fusarium vanettenii O'Donnell, Geiser, Kasson \& T. Aoki, Index Fungorum 440: 5 (2020)

Fusarium venezuelense O'Donnell, Geiser, Kasson \& T. Aoki, Index Fungorum 440: 5 (2020)

Fusarium waltergamsii $\mathrm{O}$ 'Donnell, Geiser \& $\mathrm{T}$. Aoki, Index Fungorum 440: 5 (2020)

Fusarium yamamotoi O'Donnell, Geiser, Kasson \& T. Aoki, Index Fungorum 440: 5 (2020)

Guarroa M. Calduch, Gené, Heredia \& R.F. Castañeda, Mycol. Progr. 19 (1): 22 (2020)

*Helminthosporium matsushimae D.W. Li, K. Zhang \& R.F. Castañeda, Mycotaxon 135 (4): 780 (2020)

*Heterophoma verbasci-densiflori L.W. Hou, L. Cai \& Crous, Stud. Mycol. 96: 329 (2020) Inocybe gilibertoi Para, Micol. Veg. Medit. 34: 135 (2020)

Inocybe perlucida Bandini \& E. Ferrari, Mycol. bavarica 20: 19 (2020)

Lactarius cyanotinctus Para, Micol. Veg. Medit. 34: 135 (2020)

Leucocoprinus martinicensis Blanco-Dios, Index Fungorum 449: 1 (2020)

Melanomma populicola Crous \& R.K.
Schumach., Fungal Systematics and Evolution 6: 201 (2020)

Mimicoscypha mimica T. Kosonen, Huhtinen \& K. Hansen, Persoonia 46: 53 (2020)

Neoechinodiscus Molinari \& R. Sierra, Opuscula Philolichenum 19: 172 (2020)

Ophiocordyceps desmidiospora J.P.M. Araújo \& Saltamachia, Mycologia 112: 1176 (2020)

Orbilia cookei Baral, Monogr. Orbiliomycetes: 807 (2020)

Pannaria melanesica A. Elvebakk, Australas. Lichenol. 87: 53 (2020)

Parmotrema elixii A.A. Spielm. \& Marcelli, Plant and Fungal Systematics 65 (2): 436 (2020)

Patellaria argentina Baral, Index Fungorum 454: 2 (2020)

Phoma cucumeris Bedlan, Stapfia 112: 220 (2020)

Psathyrella mollipluvisylvae Dibán \& Voto, Rivista Micologica Romana, Boll. AMER 110 (2): 91 (2020)

Psathyrella rawlae Voto, Boll. Assoc. Micol. Ecol. Romana 109 (1): 13 (2020)

Pseudocercospora kakiigena U. Braun, Fungal Systematics and Evolution 6: 114 (2020)

Psilocybe gartzii Blanco-Dios, Index Fungorum 449: 1 (2020)

Pyrenopeziza cookei Baral, Monogr. Orbiliomycetes: 1684 (2020)

Talaromyces striatoconidius Houbraken, Frisvad \& Samson, Stud. Mycol. 95: 91 (2020)

Thekopsora pseudoagrimoniae Aime \& McTaggart, Fungal Systematics and Evolution 7: 34 (2020)

Thermoascus yaguchii Houbraken, Frisvad \& Samson, Stud. Mycol. 95: 91 (2020)

Tricholoma corneri Blanco-Dios, Index Fungorum 449: 2 (2020)

Tricholoma kuthanii Blanco-Dios, Index Fungorum 449: 2 (2020)

Tricholoma luteolamellatum Blanco-Dios, 
Index Fungorum 449: 2 (2020)

Tricholoma victorianum Blanco-Dios, Index Fungorum 449: 2 (2020)

*Varioseptispora apicalis L. Qiu, Jian Ma, R.F. Castañeda \& X.G. Zhang, Mycotaxon 135
(4): 755 (2020)

Zasmidium diospyri-hispidae U. Braun, Fungal Systematics and Evolution 6: 120 (2020)

\section{不合法名称 Illegitimate names}

Biatora marmorea T. Sprib., Lichenologist 52 (2): 89 (2020)

Diaporthe salinicola Dayarathne, Mycosphere 11 (1): 91 (2020)
Neopyrenochaeta chiangraiensis Mapook \& K.D. Hyde, Fungal Diversity 101: 57 (2020)

*Russula leucocarpa G.J. Li \& C.Y. Deng, Mycosystema 39 (4): 624 (2020)

\section{不合格名称 Invalid names}

Alternaria ashwinii A.K. Mall, V. Misra, M. Kumar \& D. Pathak, Archives of Phytopathology and Plant Protection 10.1080/03235408.2020.1824378: 5 (2020)

Alternaria dilkushana A.K. Mall, V. Misra, M. Kumar \& D. Pathak, Archives of Phytopathology and Plant Protection 10.1080/03235408.2020.1824378: 6 (2020)

*Amanita lacerosquamosa Hai J. Li \& Kai Ping Zhang, Phytotaxa 456 (1): 98 (2020)

Anthodidymella Phukhams., Camporesi \& K.D. Hyde, Fungal Diversity 10.1007/s13225-020-00448-4: 21 (2020)

Anthodidymella ranunculacearum Phukhams., Camporesi \& K.D. Hyde, Fungal Diversity 10.1007/s13225-02000448-4: 27 (2020)

Aspergillus gaarensis Al-Bedak \& Moubasher, Stud. Fung. 5 (1): 62 (2020)

Biscogniauxia destructiva Vujanovic, Microorganisms 8 (12, no. 1999): 9 (2020)

Caliciopsis moriondi N. Luchi, D. Migliorini \& A. Santini, MycoKeys 73: 96 (2020)

Candida metrosideri J. Klaps, C. de Vega, C.M. Herrera, R.R. Junker, B. Lievens \& S. Álvarez-Pérez, PLoS ONE 15 (10): e0240093, 11 (2020)

Candida ohialehuae J. Klaps, C. de Vega, C.M.
Herrera, R.R. Junker, B. Lievens \& S. Álvarez-Pérez, PLoS ONE 15 (10): e0240093, 11 (2020)

*Capnodium paracoartatum Q. Tian, W.J. Li \& K.D. Hyde, Fungal Diversity 100: 372 (2020)

*Cavenderia basinodulosa Cavender, J.C. Landolt, A. Perrigo, Vadell, Pu Liu \& S.L. Stephenson, Mycologia 112 (5): 1028 (2020)

*Cavenderia canoespora Cavender, J.C. Landolt, A. Perrigo, Vadell, Pu Liu \& S.L. Stephenson, Mycologia 112 (5): 1031 (2020)

Colletotrichum noveboracense F. Khodadadi, P.L. Martin, V.P. Doyle, \& J.B. Gonzalez \& S.G. Aćimović, Scientific Reports 10 (no. 11043): 7 (2020)

*Colletotrichum philodendricola L.H. Xue, C.J. Li \& Y.W. Zhang, Pl. Dis. 104 (10): 2578 (2020)

*Colletotrichum pseudoboninense L.H. Xue \& C.J. Li, Pl. Dis. 104 (10): 2578 (2020)

Cortinarius balteatoindicus Dima, Semwal, V. Papp, Brandrud \& V.K. Bhatt, Persoonia 44: 375 (2020)

Cortinarius ulkhagarhiensis Dima, Semwal, V. Papp, Brandrud \& V.K. Bhatt, Persoonia 44: 377 (2020)

Diaporthe krabiensis Dayarathne, Mycosphere 
11 (1): 92 (2020)

*Exophiala macquariensis C.D. Zhang, N. Sirijovski, L. Adler \& B.C. Ferrari, Fungal Biology 124 (11): 153 (2020)

*Heterostelium radiatum Cavender, J.C. Landolt, A. Perrigo, Vadell, Pu Liu \& S.L. Stephenson, Mycologia 112 (5): 1033 (2020)

*Heterostelium versatile Cavender, J.C. Landolt, A. Perrigo, Vadell, Pu Liu \& S.L. Stephenson, Mycologia 112 (5): 1039 (2020)

Kazachstania bozae Gouliamova \& Dimitrov, Comptes Rendus de l'Académie Bulgare des Sciences 73 (1): 53 (2020)

Kazachstania chrysolinae Gouliamova \& Dimitrov, Comptes Rendus de l'Académie Bulgare des Sciences 73 (1): 52 (2020)

Magnuscella marina Y.S. Anteneh, M.H. Brown \& C.M.M. Franco, BioMed Research International 2019 (no. 3456164): 3 (2020)

Marasmius vagus Guard, M.D. Barrett \& Farid, Persoonia 44: 415 (2020)

Miraculales Buaya \& Thines, Philipp. J. Syst. Biol. 14 (1): 5 (2020)

Neoleptosporella camporesiana R.H. Perera \& K.D. Hyde, Fungal Diversity 100: 219 (2020)

Olegblumia demissa S.Y. Kondr., Lőkös, Jung Kim, A.S. Kondr., S.O. Oh \& Hur, Acta bot. hung. 62 (3-4): 365 (2020)

Paralophiostoma V.V. Sarma \& M. Niranjan,
Mycosphere 11 (1): 1943 (2020)

Paralophiostoma hysterioides M. Niranjan \& V.V. Sarma, Mycosphere 11 (1): 1943 (2020) Paralophiostomataceae V.V. Sarma \& M. Niranjan, Mycosphere 11 (1): 1942 (2020)

*Penicillium ullengdoense D.H. Choi \& J.G. Kim, Mycobiology 49 (1): 48 (2020)

\section{Pseudohelminthosporium clematidis}

Phukhams. \& K.D. Hyde, Fungal Diversity 10.1007/s13225-020-00448-4: 59 (2020)

*Raperostelium stabile Cavender, J.C. Landolt, A. Perrigo, Vadell, $\mathrm{Pu}$ Liu \& S.L. Stephenson, Mycologia 112 (5): 1032 (2020)

*Scleroderma venenatum Y.Z. Zhang, C.Y. Sun \& Hai J. Li, Phytotaxa 438 (2): 113 (2020)

*Scleroderma venenatum var. macrosporum

Y.Z. Zhang, C.Y. Sun \& Hai J. Li, Phytotaxa 438 (2): 114 (2020)

Sporormurispora paulsenii D. Pem, Gafforov \& K.D. Hyde, Fungal Diversity 10.1007/s13225-020-00461-7: 39 (2020)

Sticta andina Moncada, Lücking \& Lumbsch, Plant and Fungal Systematics 65 (1): 101 (2020)

Sticta arachnosylvatica Moncada, Lücking \& Lumbsch, Plant and Fungal Systematics 65 (1): 106 (2020) 